\title{
Identification and Characterization of Soybean Pigmentation Genes and Metabolic Engineering Seed Coat Color to Enable Visual Identification of Transgenic Grains
}

Nikola Kovinich

Thesis submitted to the

Faculty of Graduate and Postdoctoral Studies

In partial fulfillment of the requirements for Ph.D. degree in

Biology with specialization in Biotechnology.

Ottawa-Carleton Institute of Biology, Carleton University

(C) Nikola Kovinich, Ottawa, Canada, 2011 


\begin{tabular}{|c|c|}
\hline $\begin{array}{l}\text { Library and Archives } \\
\text { Canada }\end{array}$ & $\begin{array}{l}\text { Bibliothèque et } \\
\text { Archives Canada }\end{array}$ \\
\hline $\begin{array}{l}\text { Published Heritage } \\
\text { Branch }\end{array}$ & $\begin{array}{l}\text { Direction du } \\
\text { Patrimoine de l'édition }\end{array}$ \\
\hline $\begin{array}{l}395 \text { Wellington Street } \\
\text { Ottawa ON K1A ON4 } \\
\text { Canada }\end{array}$ & $\begin{array}{l}\text { 395, rue Wellington } \\
\text { Ottawa ON K1A ON4 } \\
\text { Canada }\end{array}$ \\
\hline
\end{tabular}

Your file Votre référence

ISBN: 978-0-494-83230-1

Our file Notre référence

ISBN: 978-0-494-83230-1

NOTICE:

The author has granted a nonexclusive license allowing Library and Archives Canada to reproduce, publish, archive, preserve, conserve, communicate to the public by telecommunication or on the Internet, loan, distribute and sell theses worldwide, for commercial or noncommercial purposes, in microform, paper, electronic and/or any other formats.

The author retains copyright ownership and moral rights in this thesis. Neither the thesis nor substantial extracts from it may be printed or otherwise reproduced without the author's permission.
AVIS:

L'auteur a accordé une licence non exclusive permettant à la Bibliothèque et Archives Canada de reproduire, publier, archiver, sauvegarder, conserver, transmettre au public par télécommunication ou par l'Internet, prêter, distribuer et vendre des thèses partout dans le monde, à des fins commerciales ou autres, sur support microforme, papier, électronique et/ou autres formats.

L'auteur conserve la propriété du droit d'auteur et des droits moraux qui protège cette thèse. $\mathrm{Ni}$ la thèse ni des extraits substantiels de celle-ci ne doivent être imprimés ou autrement reproduits sans son autorisation.
In compliance with the Canadian Privacy Act some supporting forms may have been removed from this thesis.

While these forms may be included in the document page count, their removal does not represent any loss of content from the thesis.
Conformément à la loi canadienne sur la protection de la vie privée, quelques formulaires secondaires ont été enlevés de cette thèse.

Bien que ces formulaires aient inclus dans la pagination, il n'y aura aucun contenu manquant.

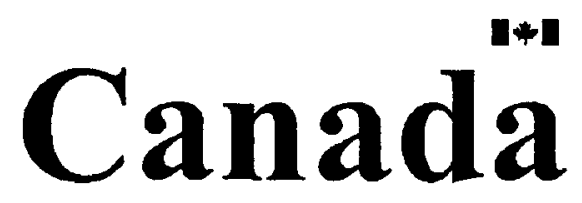




\section{ABSTRACT}

The inability to effectively detect and quantify the levels of adventitious presence of genetically modified (GM) plant grains in non-GM shipments represents a key issue that threatens the adoption of GM commodities in the worldwide market. A potentially safe and economical solution for effective detection is to produce distinct colors in GM grains of natural products by metabolic engineering. Successful attempts to engineer colored GM grains have been limited to the overexpression of plant pigment (anthocyanin) transcription factors in Arabidopsis, tobacco, and maize. The question of our research was whether soybean grain color could be manipulated by the suppression of late-stage pigment genes. The potential benefit of this approach would be to impart less unintended effects on the plant system than the induction of numerous genes by overexpression of a transcription factor. Towards testing our hypothesis, I first identified the anthocyanin branchpoint gene UGT78K1 encoding UDPglycose:flavonoid-3-O-glycosyltransferase (UF3GT) from the black soybean seed coat and demonstrated its encoded activity in vitro, and by complementation of the Arabidopsis ugt78d2 knock-out mutation. Subsequently, I identified a second UF3GT gene (UGT78K2) and the gene OMT5 coding anthocyanin-3'-Omethyltransferase (AOMT) from the black seed coat by comparing metabolite and transcriptome data between isogenic black and brown soybean seed coats differing in alleles of the $R$ locus. The proanthocyanidin (PA) branchpoint genes ANR1 and ANR2 coding anthocyanidin reductase (ANR) enzymes were then isolated from the brown soybean seed coat using RACE and the activities of their 
encoded enzymes were demonstrated in vitro. In the latter two studies we used liquid chromatography- tandem mass spectrometry (LC-MS/MS) and microarray and/or quantitative (q)RT-PCR for combined analyses of seed coat metabolite and gene expression data from brown, black, and red-brown soybean genotypes. From this data we hypothesized and confirmed that a red-brown soybean grain color could be engineered by the simultaneous suppression of ANR1 and ANR2 using RNA interference (RNAi) in brown soybean. The underlying mechanism was identified to involve positive feedback and feedforward mechanisms in the flavonoid pathway to redirect metabolic flux into anthocyanin biosynthesis. These results represent a novel approach to engineering pigmentation in plant tissues. 
Dedicated to

My grandmother, Ildiko Kovinich 


\section{ACKNOWLEDGEMENTS}

I deeply thank my supervisor Brian Miki for providing me a graduate research position in his lab. Brian has been encouraging, supportive, educating, and has provided me ample opportunities to meet and interact with people from the research community and from industry. It is Brian's conviction in me that has led me to succeed where others thought I would fail.

I am also very grateful to John Thor Arnason for being an exceptional mentor and for treating me as if I were one of his own students. The research conducted in this thesis was multi-disciplinary requiring extensive analysis in biochemistry and molecular biology. Thor provided me education, guidance, and full access to his specialized instruments that were essential to conduct the biochemical analyses.

My friend Ammar Saleem provided extensive training on specialized instruments and made himself available at all hours of the day, night, and weekends to help conduct analyses. For this, I am extremely grateful.

I thank Vincenzo De Luca, Ashraff Abdeen, and my PhD advisory committee, Owen Rowland and Tim Xing, for the very interesting meetings and discussions that helped shape my thesis projects.

For technical assistance at the AAFC I thank Tara Rintoul and Dan Brown for conducting soybean transformations, Hélène Labbé for constantly keeping me supplied and equipped in the lab, Robert Reid for cultivating soybeans, Ming $\mathrm{Hu}$ for assembling some binary vectors for transfer into Arabidopsis thaliana, Frances Tran for teaching me SDS-PAGE, and Bill Collins for his helpful advice and criticisms.

I acknowledge the financial support of Ontario Graduate Scholarship, the International Association for Plant Biotechnology - Canadian Scholarship, the Phytochemical Society of North America - Best Presentation Award.

Last but not least, I thank my family. My parents, Vuk and Germaine have provided unparalleled emotional, intellectual, and financial support. My grandmother, Ildiko has encouraged me from the time I was a child to be inquisitive and to value education. I thank my fiancée, Jia Hui (Sally) Lin, for being encouraging, emotionally supportive, and for tolerating me working countless hours in the lab. And I thank the rest of my family for their much needed friendships. I am very fortunate to share this accomplishment with such a wonderful family!

This graduate research experience has been a life-changing experience, and I am tremendously grateful to all of you for making it happen! 


\section{PREFACE}

Brian Miki, Vince De Luca and John T. Arnason invented the concept of 'coloring GM soybeans with anthocyanins'. Brian Miki and John T. Arnason obtained the funding for the research. Ammar Saleem had a primary role in the development of HPLC-DAD and LC-MS/MS methods for flavonoid analysis. Tara Rintoul and Dan Brown transferred DNA into soybeans. Nikola Kovinich invented the approach of suppressing ANR genes to redirect metabolic flux into anthocyanin biosynthesis for coloring soybeans, designed and conducted all experiments in genetics, molecular biology, and biochemistry, analysed data, and wrote the manuscripts for publication.

Each data chapter of this PhD thesis represents a manuscript that has been published, in press, or submitted for publication. The materials have been included in the thesis with kind permissions from Elsevier, Springer Science and Business Media, and BioMed Central publishing companies. We encourage readers to reference these published works directly as indicated in the corresponding chapter. 


\section{TABLE OF CONTENTS}

Abstract

Dedication

Acknowledgements

Preface

Table of contents

List of figures

xiii

List of tables

List of abbreviations

$x v i$

\section{Chapter 1:}

General introduction

1.1. Thesis rationale

1.2. Background

1.3. Hypotheses

1.4. Objectives

\section{Chapter 2:}

\section{Methods overview}

2.1. Molecular biology methods

\section{Chapter 3:}

Identification of a UDP-glucose:flavonoid 3-O-glucosyltransferase from the seed coat of black soybean (Glycine $\max (\mathrm{L}$.$) Merr.)$

3.1. Introduction

3.2. Materials and methods

3.2.1. Chemicals

3.2.2. Plant materials, growth conditions, and nucleic acid isolation

3.2.3. Cloning of the UF3GT CDNA from black soybean

3.2.4. Cloning of the UF3GT gene from black soybean

3.2.5. Phylogenetic Analyses 
3.2.6. $R T$-PCR 14

3.2.7. Recombinant protein expression in Escherichia coli 14

3.2.8. Recombinant Enzyme Assays $\quad 15$

3.2.9. Enzyme Kinetics 16

3.2.10. Enzyme specificity 16

3.2.11. Transgenic lines $\quad 17$

3.2.12. HPLC-DAD and HPLC-ESI-MS analyses 18

3.2.13. Metabolite Analysis $\quad 19$

3.3. Results 19

3.3.1. Cloning and analysis of a glycosyltransferase gene from 19 the seed coat of black soybean

3.3.2. Genomic and phylogenetic analyses of soybean 20 glycosyltransferases

3.3.3. Expression analysis of black soybean glycosyltransferases 25

3.3.4. UGT78K1 encodes a UF3GT with strict specificity for 27 anthocyanidins and flavonols in vitro

3.3.5. UGT78K1 functions as a 3-O-glucosyltransferase in

33 anthocyanin and flavonol biosynthesis in vivo

3.4. Discussion

36

3.5. Concluding remarks 42

\section{Chapter 4:}

A combined analysis of transcriptome and metabolite data to identify pigment genes and to understand the molecular control of black soybean grain color

4.1. Introduction 44

4.2. Materials and methods 48

4.2.1. Chemicals 48

4.2.2. Plant Materials and Growth Conditions $\quad 49$

4.2.3. Seed Coat Metabolite Analyses 48

4.2.4. Microarray Analysis, Semi-qRT-PCR, and qRT-PCR 50

4.2.5. Cloning of UGT78K2 and OMT5 cDNAs from the Seed 53 Coat of Black Soybean

4.2.6. Expression of Recombinant Proteins in E. coli 53

4.2.7. Recombinant Enzyme Assays 54

4.2.8. HPLC-DAD and HPLC-MS/MS Analyses 54 
4.3. Results 56

4.3.1. $q R T-P C R$ indicates differential expressions of 56 anthocyanin and proanthocyanidin genes in nearlyisogenic black (iRT) and brown (irT) soybean seed coats

4.3.2. Combined analysis of targeted and non-targeted methodologies indicate overaccumulation of anthocyanins, altered procyanidin, and reduced flavonol, benzoic acid, and isoflavone compositions in the seed coat of black (iRT) soybean

4.3.3. Microarray Analysis Indicates Concomitant Upregulation of Specific Phenylpropanoid, Flavonoid, and Anthocyanin Isogenes and Downregulation of Other Flavonoid, Benzoic Acid, and Isoflavone Isogenes in the Black (iRT) Seed Coat Transcriptome

4.3.4. Putative Assignment of Function to Genes

Differentially-Regulated between Black (iRT) and Brown (irT) Seed Coats

4.3.5. UGT78K2 and OMT5 Code UDP-Glycose:Flavonoid 3-OGlycosyltransferase and Anthocyanin 3'-OMethyltransferase Proteins, Respectively

4.4. Discussion

4.5. Concluding remarks

\section{Chapter 5:}

Biochemical analyses to understand red-brown soybean seed coat color and the identification of proanthocyanidin genes

5.1. Introduction

92

5.2. Materials and methods 97

5.2.1. Chemicals 97

5.2.2. Plant materials, growth conditions, and nucleic acid 97 isolation

5.2.3. Metabolite analysis and LC-MS/MS 98

5.2.4. Cloning ANR1 and ANR2 cDNAs 99

5.2.5. Cloning ANR1 genes 100

5.2.6. Phylogenetic analyses 100

5.2.7. RT-PCR and qRT-PCR 100 
5.2.8. Recombinant protein expression in Escherichia coli 101

5.2.9. Recombinant enzyme assays and kinetics 102

5.3. Results 103

5.3.1. The seed coats of three red-brown soybean varieties 103 have reduced amounts of soluble proanthocyanidins (Pas) compared brown soybean

5.3.2. Identification of ANR1 and ANR2 genes from the G. Max seed coat

5.3.3. Red-brown soybean varieties $M 11$ and $K D$ have nonsynonymous substitutions in the ANR1 gene that confer minor reductions in enzyme activity

5.3.4. Red-brown soybean seed coats of varieties $A g$ and $K D$ have reduced ANR1 transcript levels

5.4. Discussion

5.5. Conclusion

\section{Chapter 6:}

Coloring genetically modified soybean grains with anthocyanins by the suppression of proanthocyanidin genes

6.1. Introduction

6.2. Materials and methods

6.2.1. Chemicals

6.2.2. Plant materials and growth conditions

6.2.3. Nucleic acid isolation, vector construction, soybean

6.2.4. $q R T-P C R$

6.2.5. Metabolite Analysis

6.3. Results

6.3.1. Engineering red-brown soybean seed coats using the RNAi construct ANR-RNAi

6.3.2. High-level suppression of ANR1 and ANR2 genes by the ANR-RNAi construct confers a red-brown grain phenotype while low-level suppression fails to modify seed coat color

6.3.3. Red-brown transgenic grains undergoing high-level suppression of ANR1 and ANR2 genes have major reductions in the amount of Pas along with elevated levels 
of cyanic pigments in the seed coat

6.3.4. High-level suppression of soybean ANR1 and ANR2

genes redirects metabolic flux from $P A$ biosynthesis

into the anthocyanin and flavonol-3-O-glucoside

pathways

6.3.5. High-level suppression of soybean ANR1 and ANR2

genes causes the upregulation of anthocyanin and

flavonol-3-O-glucoside isogene transcripts

6.4. Discussion

6.5. Concluding remarks

\section{Chapter 7:}

General discussion

7.1. How does this study fit in existing societal, methodological and theoretical debates?

7.1.1. The societal debate

7.1.2. The methodological debate

7.1.3. The theoretical debate

7.2. Original contributions

7.3. Future research

7.4. Implications and concluding remarks

References

Appendix A. Primers

Appendix B. Developmental properties of black (iRT) and brown (irT)

soybean seed coats at the $300-400 \mathrm{mg}$ fresh seed weight stage

Appendix C. Proanthocyanidin (PA) subunit compositions, degree of polymerizations, and amounts from the seed coats of black ( $R T T$ ) and brown (irT) soybean Clark isolines

Appendix D. Gene probe sets up-regulated more than 2-fold in the seed coat of black (iRT) soybean compared to the brown (irT) isoline 
Appendix E. Distribution of gene function categories of probe sets that were up-regulated more than 2-fold in the seed coat of black (iRT) soybean relative to the seed coat of brown (irT) soybean

Appendix F. Glycine max UGTs

Appendix G. Glycine max OMTs

Appendix H. Gene probe sets downregulated more than 2-fold in the seed coats of black (iRT) soybean compared to the seed coats of brown (irT) soy- bean at the $300-400 \mathrm{mg}$ fresh seed weight stage of development

Appendix I. Distribution of gene function categories of probe sets that were down-regulated more than 2-fold in the seed coat of black ( $i R T$ ) soybean relative to the seed coat of brown (irT) soybean

Appendix J. Genes located on chromosome 9 of the soybean genome sequence Glyma1 that are associated with probe sets differentially-regulated more than 2-fold in the seed coat of black (iRT) soybean variety Clark

Appendix K. Semi-qRT-PCR validation of the expressions of select genes found to be differentially expressed in black ( $i R T$ ) and brown (irT) seed coats by microarray analysis

Appendix L. Alignment of G. max UG3GT proteins UGT78K2 and 198 UGT78K1 from variety Clark using the ClustalW program with default parameters. Amino acid differences are shown with grey background

Appendix M. Primers

Appendix N. Primers

Appendix 0 . Primers

Appendix P. Multiple reacton monitoring specifications for quantificaton of differentially accumulated flavonoid compounds from the seed coats of transgenic soybeans expressing the ANR-RNAi construct using a 3200 QTRAP

Appendix Q. Developmental properties of T2 lines compared to the untransformed control (ir) at two stages of development

Appendix R. Percent sequence similarity of ANR1-RNAi to RED 205 superfamily genes 


\section{List of Figures}

Figure 1.1 Flavonoid biosynthesis in the soybean seed coat. 3

Figure 3.1 Genomic organisations of UGT78K1 (Glyma07g30180), 22 Glyma08g07130 and Glyma07g30190 from Glycine max and Arabidopsis thaliana glycosyltransferases UGT78D2 (At5g17050), At5g17040, and At5g17030

Figure 3.2 Unrooted phylogenetic tree of UGT78K1 and flavonoid glycosyltransferase amino acid sequences

Figure 3.3 Transcript detection of UGT78K1 (Glyma07g30180), Glyma08g07130, Glyma07g30190, and Glyma07g30200 by RT-PCR with gene-specific primers

Figure 3.4 Purification of his-tagged UGT78K1 and identification of reaction products

Figure 3.5 UGT78K1 kinetics for cyanidin and kaempferol acceptor substrates. Reaction kinetics for acceptor substrates kaempferol and cyanidin using $5 \mathrm{mM}$ UDPG as a sugar donor

Figure 3.6 Activities of UGT78K1 for anthocyanidins and flavonols

Figure 3.7 The UGT78K1 gene complements anthocyanin biosynthesis in the Arabidopsis ugt78d2 mutant

Figure 3.8 Functional analysis of the UGT78K1 gene by complementation of the Arabidopsis ugt78d2 null mutation

Figure 4.1 Transcript profiles from black (iRT) and brown (irT) seed coats during development measured by qRT-PCR

Figure 4.2 Anthocyanin accumulations in black (iRT) and brown (irT) soybean seed coats during seed development

Figure 4.3 Anthocyanins from the seed coat of black (iRT) soybean variety Clark. Numbers correspond to chromatographic peaks identified by HPLC-DAD

Figure 4.4 Distribution of compound classes from black (iRT) and brown (irT) soybean seed coats identified by LC-MS/MS

Figure 4.6 Summary of flavonoid/phenylpropanoid gene and metabolite accumulations from black and brown soybean seed coats

Figure 4.7 Purification of his-tagged UGT78K2 and determination of the reaction product and kinetics for cyanidin 
Figure 4.8 Purification of his-tagged OMT5 and determination of the reaction product for cyanidin 3-O-glucoside

Figure 4.9 Seed coat transcript profiles of UGT78K2 and OMT5 during seed development measured by qRT-PCR

Figure 5.1 Flavonoid biosynthesis in the soybean seed coat

Figure 5.2 Flavonoid amounts from the seed coats of brown $(O)$ and red- 106 brown (o) soybean varieties expressed in units $\mathrm{mg}$ flavonoid per gram lyophilized seed coat (LSC)

Figure 5.3 Phylogenetic tree of ANR and other reductase-epimerasedehydrogenase (RED) superfamily proteins

Figure 5.4 Transcript detection of ANR1 and ANR2 from Clark (ir) cDNA by RT-PCR with gene-specific primers

Figure 5.5 Purification of his-tagged ANR1 and ANR2 and identification of reaction products

Figure 5.6 Alignment of $G$. max ANR1 proteins from brown $(O)$ seed coat varieties (ir, r17, M100) and red-brown (o) seed coat varieties (Ag, KD, and M11) using the ClustalW program with default parameters

Figure 5.7 In vitro kinetics of recombinant ANR1 enzymes from brown soybean variety ir and red-brown soybean varieties M11 and $\mathrm{KD}$

Figure 5.8 Seed coat transcript profiles of PA and anthocyanin branchpoint genes measured by qRT-PCR

Figure 6.1 Simplified diagram of flavonoid biosynthesis in the seed coat of soybean variety Clark

Figure 6.2 Structure of the ANR-RNAi DNA construct and phenotypes associated with its genomic integration in soybean

Figure 6.3 qRT-PCR measurement of transcript profiles from the seed coat of transgenic soybean carrying the ANR-RNAi construct

Figure 6.4 Total PA and cyanic pigment amounts from the seed coat of transgenic soybean carrying the ANR-RNAi construct expressed in units $\mathrm{mg}$ per gram lyophilized seed coat (LSC)

Figure 6.5 Identification and quantification of seed coat metabolites that accumulate to different amounts in transgenic lines with redbrown grain color compared to the untransformed control

Figure 6.6 qRT-PCR measurement of transcript profiles identified positive feedforward/feedback induction of flavonoid gene expressions in the seed coat of transgenic soybean carrying the ANR-RNAi construct 


\section{List of Tables}

Table 3.1 Primers 13

Table 4.1 HPLC-DAD analysis of anthocyanins from the seed coat of 63 black (iRT) soybean variety Clark

Table 4.2 HPLC-MS/MS analysis of seed coat extracts of black (iRT) 66 and brown (irT) soybean

Table 4.3 Putative phenylpropanoid/flavonoid gene sets upregulated more than 2-fold in the seed coats of black (iRT) soybean compared to the brown (irT) isoline

Table 4.4 Putative phenylpropanoid/flavonoid gene probe sets downregulated more than 2-fold in the black (iRT) compared to the brown (irT) soybean seed coat

Table 5.1 Soybean lines, alleles, and phenotypic properties of their seeds

Table 5.2 Comparison of ANR1 genomic and amino acid sequences among brown $(O)$ and red-brown $(0)$ soybean varieties 


\section{List of Abbreviations}

Commonly accepted gene and protein nomenclature are used in this thesis.

Genes and their corresponding mRNAs are listed in italics. Proteins are represented by non-italiciazed uppercase letters. Family 1 glycosyltransferase (UGT) genes have approved names (Mackenzie et al., 1997).

4CL

$\mathrm{Ag}$

amu

ANR

ANR1

ANR2

ANS

ANS2

ANS3

AOMT

C3G

C3Ga

CAPS

$\mathrm{CHI}$

CHS

COC

Cy
- 4-coumarate-CoA ligase

- Red-brown saddle soybean variety Agate

- Atomic mass unit

- anthocyanidin reductase

- ANR isoform 1 from Glycine max

- ANR isoform 2 from Glycine max

- anthocyanidin synthase

- anthocyanidin synthase isoform 2 from Glycine max

- anthocyanidin synthase isoform 3 from Glycine max

- anthocyanin Omethyltransferase

- Cyanidin-3-O-glucoside

- Cyanidin-3-O-galactoside

- N-cyclohexyl-3aminopropanesulfonic acid

- chalcone isomerase

- chalcone synthase

- Coefficience of coincidence

- cyanidin 


\begin{tabular}{|c|c|}
\hline DFR & $\begin{array}{l}\text { - dihydroflavonol 4- } \\
\text { reductase }\end{array}$ \\
\hline F3'5'H & - flavonoid 3',5'-hydroxylase \\
\hline F3'H & - flavonoid 3'-hydroxylase \\
\hline F3H & - flavanone 3-hydroxylase \\
\hline FLS & - flavonol synthase \\
\hline FSW & - Fresh seed weight \\
\hline G4DT & $\begin{array}{l}\text { - pterocarpan 4- } \\
\text { dimethylallyltransferase }\end{array}$ \\
\hline GFP & - Green fluorescent protein \\
\hline GST & - glutathione S-transferase \\
\hline GST26 & $\begin{array}{l}\text { - GST isoform } 1 \text { from } \\
\text { Glycine max }\end{array}$ \\
\hline GUS & - beta-glucuronidase \\
\hline HEPES & $\begin{array}{l}\text { - 4-(2-hydroxyethyl)-1- } \\
\text { piperazineethanesulfonic } \\
\text { acid }\end{array}$ \\
\hline HPLC-DAD & $\begin{array}{l}\text { - High performance liquid } \\
\text { chromatography - diode } \\
\text { array detection }\end{array}$ \\
\hline $\boldsymbol{i}$ & $\begin{array}{l}\text { Homozygous recessive } i \\
\text { alleles for Glycine max } \\
\text { seed coat color }\end{array}$ \\
\hline I & $\begin{array}{l}\text { Homozygous dominant I } \\
\text { locus alleles for Glycine } \\
\text { max seed coat color }\end{array}$ \\
\hline I3G & $\begin{array}{l}\text { - Isorhamnetin-3-O- } \\
\text { glucoside }\end{array}$ \\
\hline IFR & - isoflavone reductase \\
\hline IMAC & $\begin{array}{l}\text { - Ion-metal affinity } \\
\text { chromatography }\end{array}$ \\
\hline ir & $\begin{array}{l}\text { - Brown soybean variety } \\
\text { Clark }\end{array}$ \\
\hline KD & $\begin{array}{l}\text { - Red-brown soybean } \\
\text { variety Kuro Daizu }\end{array}$ \\
\hline LAR & $\begin{array}{l}\text { - leucoanthocyanidin } \\
\text { reductase }\end{array}$ \\
\hline LAR1 & $\begin{array}{l}\text { LAR isoform } 1 \text { from } \\
\text { Glycine max }\end{array}$ \\
\hline
\end{tabular}




\begin{tabular}{|c|c|}
\hline LC-MS/MS & $\begin{array}{l}\text { - Liquid chromatography } \\
\text { tandem mass } \\
\text { spectrometry }\end{array}$ \\
\hline LSC & - Lyophylized seed coat \\
\hline M100 & $\begin{array}{l}\text { EMS-mutagenized brown } \\
\text { soybean variety Linia } \\
\text { N145 }\end{array}$ \\
\hline M11 & $\begin{array}{l}\text { EMS-mutagenized red- } \\
\text { brown soybean variety } \\
\text { Linia N145 }\end{array}$ \\
\hline MATE & $\begin{array}{l}\text { multidrug and toxic } \\
\text { extrusion protein }\end{array}$ \\
\hline MES & $\begin{array}{l}\text { - 2-(N-morpholino) } \\
\text { ethanesulfonic acid }\end{array}$ \\
\hline MRM & $\begin{array}{l}\text { - Multiple reaction } \\
\text { monitoring }\end{array}$ \\
\hline 0 & $\begin{array}{l}\text { Homozygous recessive o } \\
\text { locus alleles for Glycine } \\
\text { max seed coat color }\end{array}$ \\
\hline 0 & $\begin{array}{l}\text { - Homozygous dominant } O \\
\text { locus alleles for Glycine } \\
\text { max seed coat color }\end{array}$ \\
\hline OMT5 & - AOMT from Glycine max \\
\hline PA & $\begin{array}{l}\text { Proanthocyanidin (a.k.a. } \\
\text { condensed tannin) }\end{array}$ \\
\hline PEPC16 & $\begin{array}{l}\text { Phosphenol pyruvate } \\
\text { carboxylase isoform } 16 \\
\text { from Glycine max }\end{array}$ \\
\hline PSPG & $\begin{array}{l}\text { - plant secondary product } \\
\text { glucosyltransferase }\end{array}$ \\
\hline Q3G & - Quercetin-3-O-glucoside \\
\hline $\boldsymbol{r}$ & $\begin{array}{l}\text { Homozygous recessive } r \\
\text { locus alleles for Glycine } \\
\text { max seed coat color }\end{array}$ \\
\hline$R$ & $\begin{array}{l}\text { - Homozygous dominant } R \\
\text { locus alleles for Glycine } \\
\text { max seed coat color }\end{array}$ \\
\hline r17 & $\begin{array}{l}\text { - Brown soybean variety } \\
\text { Soysota }\end{array}$ \\
\hline RED & $\begin{array}{l}\text { Reductase-epimerase- } \\
\text { dehydrogenase }\end{array}$ \\
\hline
\end{tabular}




$\begin{array}{lll}\text { RNAi } & - & \text { RNA interference } \\ t & - & \text { Homozygous recessive } t \\ & \text { locus alleles for Glycine } \\ & \text { max seed coat color } \\ T & - & \text { Homozygous dominant } T \\ & \text { locus alleles for Glycine } \\ & \text { max seed coat color } \\ \text { UF3GT } & - & \text { UDP-glycose:flavonoid 3- } \\ & \text { O-glycosyltransferase } \\ \text { UGT78D2 } & - & \text { UF3GT from Arabidopsis } \\ & \text { thaliana } \\ \text { UGT78K1 } & - & \text { UF3GT isoform 1 from } \\ & \text { Glycine max } \\ \text { UGT78K2 } & - & \text { UF3GT isoform 2 from } \\ & \text { Glycine max } \\ \text { wko } & - & \text { Week-old }\end{array}$




\section{CHAPTER 1}

\section{General Introduction}

Adapted from one publication and one submitted manuscript:

Kovinich $\mathrm{N}^{1}$, Arnason $\mathrm{JT}^{2}$, De Luca $\mathrm{V}^{2}$ and Miki $\mathrm{B}^{2}$. 2011. Coloring Soybeans with Anthocyanins? Recent Advances in Phytochemistry, 41: 47-57.

Kovinich $\mathrm{N}^{1}$, Saleem $\mathrm{A}^{2}$, Rintoul $\mathrm{TL}^{3}$, Brown $\mathrm{DCW}^{5}$, Arnason $\mathrm{JT}^{4,5}$, and Miki $\mathrm{B}^{4,5}$. Submitted. Aposomatic GM soybeans: Coloring genetically modified soybean grains with anthocyanins by suppression of the proanthocyanidin genes ANR1 and ANR2. Transgenic Research.

${ }^{1}$ Designed and conducted research, analyzed data, and wrote the manuscripts for publication

${ }^{2}$ Invented the concept of 'Coloring soybeans with anthocyanins' and revised the manuscript(s)

${ }^{3}$ Developed the LC-MS/MS methods

\subsection{Thesis rationale}

The commercial production of genetically engineered (GE) crops has risen to 250 million acres worldwide (Lemaux, 2008). As the prevalence and diversity of engineered crops increase, contamination of non-modified grain with transgenics is becoming problematic (Doyle, 2008). Distinct colors for the visible identification and quantitation of transgenic materials may provide increased safety to the consumer and freedom to the producer by enabling more effective detection.

\subsection{Background}

Anthocyanins are flavonoid pigments found in plants that provide red-to-blue color to flowers, leaves, and grains. Proanthocyanidins (PAs) represent another class of flavonoid pigments that if present in plant grains provide a brown color 
when oxidized during desiccation. The biosynthesis of anthocyanins and PAs (Figure 1.1) has been well-characterized over the past several decades, with numerous structural, regulatory, and transport genes identified (reviewed by Mol et al., 1998; Winkel-Shirley, 2001; Lepiniec et al., 2006; Zhao and Dixon, 2010). However, the genes for only 4 flavonoid enzymes (CHS, F3H, F3'H, and F3'5'H) have been identified as being involved in pigment biosynthesis in the soybean seed coat (Todd and Vodkin, 1996; Toda et al., 2002; Zabala and Vodkin, 2003; Zabala and Vodkin, 2005; Zabala and Vodkin, 2007; Kovinich et al., 2010). Although often studied separately, the biosynthesis of anthocyanin and PA pigments may be intimately linked in tissues that synthesize both pigments. The anthocyanin branchpoint enzyme UDP-glycose:flavonoid-3-O-glycosyltransferase (UF3GT; EC 2.4.1.115) and the PA branchpoint enzyme anthocyanidin reductase (ANR; EC 1.3.1.77) both utilize the unstable flavonoid precursor, cyanidin, as a substrate (Figure 1.1).

Black soybean, long considered a medicinal food and consumed only occasionally in Japan and Korea, is similar in composition to cultivated yellow soybean, except that it accumulates high levels of anthocyanin and PA pigments in the seed coat (Todd and Vodkin 1993). Interestingly, the black seed coat has a red color during mid-stages of seed development due to the presence of only moderate amounts of anthocyanins. By contrast, the seed coat of brown soybean accumulates only PA pigments and browns during desiccation (Todd and Vodkin 1993). 


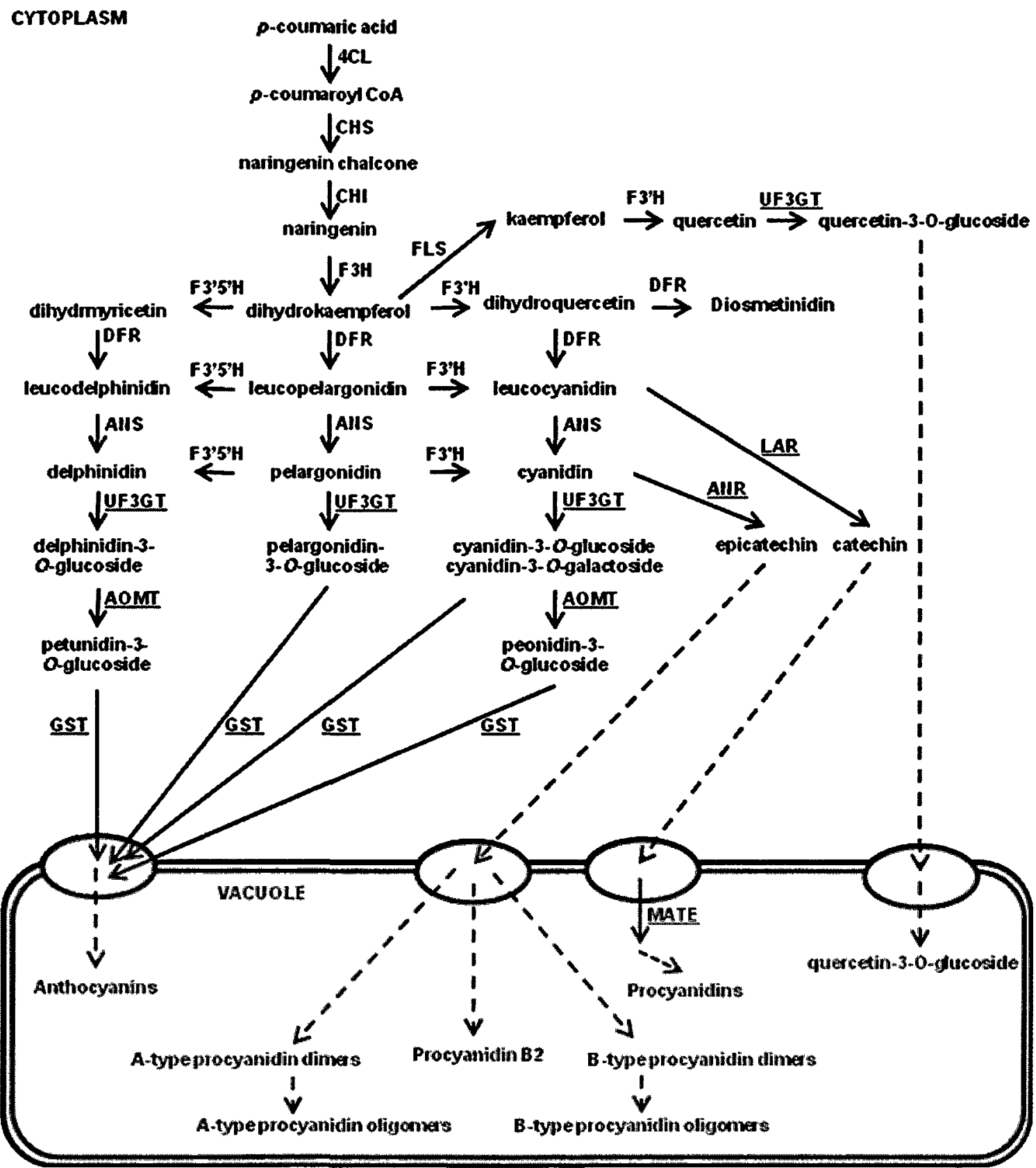

Figure 1.1. Flavonoid biosynthesis in the soybean seed coat. Proteins are in uppercase, metabolites in lowercase. Proteins with genes that have not been identified from the soybean seed coat are underlined. $4 \mathrm{CL}$, 4-coumarate-CoA ligase; $\mathrm{CHS}$, chalcone synthase; $\mathrm{CHI}$, chalcone isomerase; $\mathrm{F} 3 \mathrm{H}$, flavanone 3hydroxylase; F3' $\mathrm{H}$, flavonoid 3'-hydroxylase; F3'5' $\mathrm{H}$, flavonoid 3',5'-hydroxylase; DFR, dihydroflavonol reductase; ANS, anthocyanidin synthase; UF3GT, UDPglycose:flavonoid 3-O-glycosyltransferase; AOMT, anthocyanin Omethyltransferase; GST, glutathione S-transferase; FLS, flavonol synthase; LAR, 
leucoanthocyanidin reductase; ANR, anthocyanidin reductase; MATE, multidrug and toxic extrusion protein.

Since the first reported engineering of a "novel" flower color by the expression of a maize enzyme in petunia (Meyer et al., 1987), numerous experiments have successfully modified flower color in various plant species by manipulating flavonoid gene expressions (Tanaka et al., 2005). For example, a blue-hued rose was produced by the expression of a viola gene for flavonoid-3'-5'-hydroxylase $\left(F 3^{\prime} 5^{\prime} H\right)$, as increased hydroxylation of the anthocyanin B-ring imparts bluer color (Katsumoto et al., 2007). Another interesting example was the modification of flower color by suppression of the flavonol synthase (FLS) gene in petunia resulting in the redirection of metabolic flux into the anthocyanin pathway (Holton et al., 1993). However, successful reports of engineering novel grain color may be limited to two studies that have overexpressed anthocyanin transcription factors in Maize (Shen and Petolino, 2006), Arabidopsis, and Tobacco (Geekiyanage, 2009). The suppression of late-stage pigment biosynthesis genes for the purposes of engineering grain color may result in more predictable changes in the seed coat metabolome, and thus have lower potential for unintended effects on the biosafety of food and feed. 


\subsection{Hypotheses}

i. The suppression of the UF3GT gene(s) in the seed coat of black soybean would result in reduced anthocyanin accumulations (Figure 1.1) and produce a red-brown grain color;

ii. The suppression of the ANR gene(s) in the seed coat of brown soybean would redirect metabolic flux into the anthocyanin pathway (Figure 1.1) and produce a red grain color.

\subsection{Objectives}

i. Identify the UF3GT gene(s) from black seed coat cDNA;

ii. Determine whether the UF3GT gene(s) (or other pigment genes) have a key role in anthocyanin biosynthesis (and thus color) in the black soybean seed coat;

iii. Identify the ANR gene(s) from brown seed coat cDNA;

iv. Determine whether the ANR gene(s) (or other pigment genes such as UF3GT) control metabolic flux between anthocyanin and PA pathways (and thus color) in pigmented soybean seed coats.

v. Engineer colored soybeans by suppression anthocyanin and/or PA genes 


\section{CHAPTER 2}

\section{Methods Overview}

\subsection{Molecular biology methods}

Standard molecular biology methods were used in the research presented in this thesis with one exception. The extraction of RNA from soybean seed coats required a specific protocol (Wang et al., 1994) to remove proanthocyanidins (PAs), as these compounds bind and precipitate RNA.

\subsection{Biochemistry methods}

Standard biochemistry practices were used for metabolite extractions with one detail that could use clarification. The isolation of flavonoids from enzyme reactions (described in the data chapters) involved evaporating methanol or ethyl acetate solvents under a stream of nitrogen gas to concentrate the extract. This practice was achieved using an N-EVAP apparatus (Organomation Associates Inc. MA, USA).

The analytical methods used for the analysis of flavonoid compounds (i.e. high performance-liquid chromatography- diode array detection (HPLC-DAD) and liquid chromatography- tandem mass spectrometry (LC-MS/MS), in scanning and multiple reaction monitoring (MRM) modes) were developed specifically for the compounds analyzed. Readers should consult the data chapters for details. 


\section{CHAPTER 3}

\section{Identification of a UDP-glucose:flavonoid 3-O-glucosyltransferase from the seed coat of black soybean (Glycine max (L.) Merr.)}

\section{Adapted from:}

Kovinich $\mathrm{N}^{1}$, Saleem $\mathrm{A}^{3}$, Arnason $\mathrm{JT}^{2}$, and Miki $\mathrm{B}^{2}$. 2010. Functional characterization of a UDP-glucose:flavonoid 3-O-glucosyltransferase from the seed coat of black soybean (Glycine max (L.) Merr.). Phytochemistry 71:1253-1263.

\footnotetext{
1 Designed and conducted research, analyzed data, and wrote the manuscript for publication ${ }^{2}$ Obtained funding for the research and revised the manuscript

${ }^{3}$ Developed the HPLC-DAD method
}

\subsection{Introduction}

Black soybean (Glycine max (L.) Merr.) accumulates relatively high amounts of cyanidin-, delphinidin-, petunidin-, and pelargonidin-based anthocyanins exclusively as 3-O-glucosides in the seed coat (Choung et al., 2001; Lee et al., 2009). Minor amounts of 3-O-galactosides of cyanidin and delphinidin, and 3-Oglucosides of peonidin and a cyanidin-catechin complex have also been reported from this tissue (Lee et al., 2009). By contrast, anthocyanins from Arabidopsis contain glucosyl moieties linked to the 3- and 5-positions of the cyanidin backbone that are decorated with p-coumaroyl, malonyl, and sinapoyl groups (Tohge et al., 2005). Several genes involved in the early steps of anthocyanin and general flavonoid biosynthesis in the seed coat of black soybean have been identified, but the gene catalyzing the final step in anthocyanin biosynthesis, namely UDP-glucose:flavonoid 3-O-glucosyltransferase (UF3GT), has remained unreported (reviewed by Kovinich et al., 2011a). 
UF3GT catalyzes the transfer of glucose, from uridine diphosphate (UDP)glucose, to the 3-position of anthocyanidins to form the corresponding anthocyanins (Heller and Forkmann, 1988, 1993; Forkmann and Heller, 1999). UF3GTs belong to a large multigene family (Family 1) of inverting glycosyltransferases (UGTs) (CAZy, http://www.cazy.org/fam/acc_GT.html) defined by the presence of a conserved carboxy-terminal consensus sequence, the plant secondary product glycosyltransferase signature sequence (PSPG box) (Hughes and Hughes, 1994), which is involved in binding the UDP moiety of the sugar nucleotide (Offen et al., 2006) to the enzyme. UGT Family 1 consists of over 100 members in Arabidopsis (Ross et al., 2001) and approximately 150 members in the legume Medicago truncatula (Modolo et al., 2007). Presently, UGT substrate specificity or function cannot be predicted using amino acid sequence information alone (Modolo et al., 2007) and identity among UF3GTs is as low as $25 \%$ (Sarkar et al., 2007). Some UGTs that catalyze the transfer of glucose to the 3-position of anthocyanidins can also glucosylate flavonol (Ralston et al., 1988; Tanaka et al., 1996; Ford et al., 1998; Ogata et al., 2004; Almeida et al., 2007), dihydroflavonol, flavone, isoflavone (Owens and Mclntosh, 2009), flavanone and coumestan substrates in vitro (Modolo et al., 2007). Identification and characterization of UGTs with high amino acid identities but different biochemical activities may provide insights into structure-function relationships and is the focus of ongoing studies (Osmani et al., 2009; Owens and Mclntosh, 2009; Wang, 2009). 
Anthocyanins are believed to be synthesized on the cytoplasmic face of the endoplasmic reticulum by metabolons (Saslowsky and Winkel-Shirley, 2001) and to be transported into the vacuole by systems that remain to be characterized (Grotewold and Davis, 2008). Glucosylation of the 3-position of anthocyanidins increases their chemical stability and glucosylation of secondary metabolites generally increases their solubility and enables access to active membrane transport systems that recognize glucosylated compounds but not their aglycones (Hostel, 1981). Glucosylation of the 3-position of anthocyanidins and the structurally-similar flavonols may be required for their accumulation in some plant species, as a null mutation in the Arabidopsis UF3GT gene UGT78D2 (At5g17050) resulted in a drastic reduction of these compounds (79\%) relative to the wildtype (Tohge et al., 2005). The molecular basis of the black phenotype in soybean seed coats is not well understood, but the accumulation of high amounts of anthocyanins may be involved (Kovinich et al., 2011a). Engineering reduced anthocyanin amounts in the seed coat of black soybean by suppression of the UF3GT gene could potentially be used to produce distinct seed colors to enable the visual identification and monitoring of transgenic grains (Kovinich et al., 2011a). However, before engineering reduced anthocyanin amounts by suppression of the UF3GT gene can be attempted, the gene(s) catalyzing the final step in anthocyanin biosynthesis must be identified from the black soybean seed coat.

The purpose of this study was two-fold: 1) to identify the gene that catalyzes the final step in anthocyanin biosynthesis from the seed coat of black soybean to 
provide a possibility of engineering reduced pigment in transgenic grains, and 2) to characterize the catalytic properties of the recombinant enzyme to provide a basis for future glucosyltransferase structure-function analyses. We report here the isolation of a glycosyltransferase cDNA (UGT78K1) from the seed coat of black soybean. To determine the function of the corresponding recombinant enzyme, 28 flavonoid substrates previously identified in soybean were tested as substrates. To determine the in vivo function, the cDNA was transferred into the Arabidopsis mutant $u g t 78 d 2$ in an attempt to restore anthocyanin biosynthesis. To provide a basis for future structure-function analyses of UGT78K1, kinetics and specificity analyses were conducted with anthocyanidins and other flavonoid substrates.

\subsection{Materials and Methods}

\subsubsection{Chemicals}

Cyanidin was purchased from Indofine (Somerville, NJ, USA), delphinidin, myricetin, kaempferol, isorhamnetin, dihydroquercetin, apigeninidin, genistin, genistein and (-)-epicatechin were purchased from Sigma-Aldrich (Oakville, ON, CA), pelargonidin, malvidin, peonidin, kaempferol, quercetin, isorhamnetin and their 3-O-glucosides, petunidin, cyanidin 3-O-galactoside, (+)-catechin, apigenin from Extrasynthese (Lyon, FR) and 3-O-glucosides of delphinidin and petunidin from Polyphenols (Hanaveien, Norway). 
3.2.2. Plant materials, growth conditions, and nucleic acid isolation

Black soybean (Glycine max (L.) Merr.) cv. Clark line (PI547438) was

obtained from the U.S. Department of Agriculture Soybean Germplasm Collection (Agricultural Research Service, University of Illinois at ChampaignUrbana). Seeds were germinated in vermiculite and transferred to soil after one week. Plants were grown in a Conviron E15 cabinet under $16 / 8 \mathrm{~h}$ light/dark at 25 ${ }^{\circ} \mathrm{C}$ and $20^{\circ} \mathrm{C}$, respectively. Vegetative organs (obtained from 3 wko seedlings), seed coats, embryos and flower buds were dissected from plants, immediately frozen in liquid nitrogen, and stored at $-80^{\circ} \mathrm{C}$. DNA was isolated from trifoliate unexpanded soybean leaves or Arabidopsis seedlings using the DNeasy Plant Mini Kit (QIAGEN). RNA was isolated from all tissues using the RNeasy Plant Mini Kit (QIAGEN) or from black soybean seed coats as described previously (Wang and Vodkin, 1994).

Arabidopsis lines used in this study were of the Columbia ecotype. The TDNA insertion line ugt78d2 (CS25064) was obtained from the SALK collection (Alonso et al., 2003) at the ABRC (Columbus, OH, USA). Seeds were surfacesterilized in $2 \%$ Triton $/ 70 \% \mathrm{EtOH}(\mathrm{v} / \mathrm{v})$ for $5 \mathrm{~min}$ on a mixer wheel, rinsed thrice with $\mathrm{EtOH}$, dried, and plated on culture media (1X Gamborg's salts, $\mathrm{pH} 5.8,1 \mathrm{X}$ B-5 vitamins, sucrose $(2 \%, w / v)$, agar $(0.8 \%, w / v)$ and $10 \mathrm{mg} \mathrm{ml}^{-1}$ benomyl). To release seed dormancy plates were incubated for $3 \mathrm{~d}$ at $4{ }^{\circ} \mathrm{C}$, then transferred to light. Germination and seedling culture occurred in a Conviron I23L incubator (Conviron, Winnipeg, Canada) at $22^{\circ} \mathrm{C}$, under cool-white fluorescent lights (117 
$\left.\mu \mathrm{E} \cdot \mathrm{m}^{2} \cdot \mathrm{s}\right)$ with a photoperiod of $16 \mathrm{~h}$ light to favor vegetative growth. Seedlings were harvested for nucleic acid isolation or transferred to soil after 2 weeks.

\subsubsection{Cloning of the UF3GT cDNA from black soybean}

By searching the NCBI Glycine max EST collection using the megablast algorithm and the Clitoria ternatea Ct3GT-A mRNA (AB185904, N. Noda, Unpublished) as a query, forward and reverse gene-specific primers (B1F, B1R, B2F. B2R, E1F, E1R, M1F, M1R) (see Table 3.1) were designed to amplify partial UGT78K1 sequences from seed coat CDNA using 5' and 3' RACE (CLONTECH), respectively. The resulting partial cDNA fragments were cloned into the TOPO-TA vector (Invitrogen) and sequenced. The full-length coding region was then amplified from seed coat cDNA by end-to-end PCR using primers UHF/UHR (Table 3.1) and the Pfx50 DNA polymerase (Invitrogen). The resulting $1517 \mathrm{bp}$ amplicon was cloned into the $\mathrm{Ndel}$ and $\mathrm{BamHI}$ sites of the pET-14b vector (Novagen, ON, Canada) and sequenced to confirm its identity.

\subsubsection{Cloning of the UF3GT gene from black soybean}

The UGT78K1 sequence was amplified from the soybean genome by PCR using primers UHF/UHR (Table 3.1) and the Pfx50 DNA polymerase (Invitrogen), cloned into the pENTR/D-TOPO vector (Invitrogen), and sequenced. PCR of the wild type UGT78D2 allele, the T-DNA insertion ugt78d2, and the UGT78K1 gene was performed with primer pairs ULP/URP, LB/URP, and UNSF/UNSR, respectively (Table 3.1$)$. 
Table 3.1: Primers.

\begin{tabular}{|c|c|}
\hline Primer & Sequence \\
\hline AtTubF & 5'-GGGCTAAAGGACACTACACTGAAGG-'3 \\
\hline AtTubR & 5'-ССTCCTGCACTTCCACTTCGTCTTC-'3 \\
\hline $\mathrm{B} 1 \mathrm{~F}$ & 5'-TGTTCCACTTCCTTCCACGTTGTTGC-'3 \\
\hline B1R & 5'-CACTTTCTTCCAATGCCTCTGCCACTG-'3 \\
\hline $\mathrm{B} 2 \mathrm{~F}$ & 5'-GGCTTATGTTTGCTTTGGGACTGTGGTG-'3 \\
\hline $\mathrm{B} 2 \mathrm{R}$ & 5'-CACTATTTTCCCACGCTTCTTGGTCCTC-'3 \\
\hline${ }^{t \ddagger} B U F$ & 5'-CACCGGATCCACCATGGATCATCAAAACAAACACGTAGCA-'3 \\
\hline${ }^{\dagger} \mathrm{BUR}$ & 5'ACTCGAGCTCATCGCCGACTTATTTTAGGATCTTGTCG-'3 \\
\hline E1F & 5'-CTTTGGCAGCCACCTTATGCCTCTCTTA-'3 \\
\hline E1R & 5'-AGTTGGGTTTTTTGCCTAGGACGTGACC-'3 \\
\hline GmTubF & 5'-GTGACTTGAACCATCTGATCTAGC-3' -'3 \\
\hline GmTubR & 5'-GTTGAAGCCATCCTCAAGCCAG-3' -'3 \\
\hline LB & 5'-GGACCGCTTGCTGCAACT-'3 \\
\hline M1F & 5'-AGACACAGATGCAACAGGGTGCTTGTC-'3 \\
\hline M1R & 5'-GAGACCAAAGAAAGGGAACACCACTTGC-'3 \\
\hline t\#UHF & 5'-CACCCATATGGATCATCAAAACAAACACGTAGCA-'3 \\
\hline †UHR & 5'-ACTCGGATCCATCGCCGACTTATTTTTAGGATCTTGTCG-'3 \\
\hline ULP & 5'-CTCTTCGTTATTTTTCCTCCGG-'3 \\
\hline URP & 5'-TCAAACCCATCTTTCGTGAAG-'3 \\
\hline UNSF & 5'-TGGATCATCAAAACAAACACGTAGCAGT-'3 \\
\hline UNSR & 5'-GTGCTGGCGAATCAAGTCAGTGTAGA-'3 \\
\hline
\end{tabular}

${ }^{\dagger}$ Restriction site in bold

${ }^{\ddagger}$ Start codon italicized bold 


\subsubsection{Phylogenetic Analyses}

A multiple alignment of the deduced amino acid sequences of UGT78K1 and other flavonoid glycosyltransferases was constructed using ClustalW (http://www.align.genome.jp). Node support was estimated by parsimony bootstrap analysis (1000 bootstrap replicates, 10 random addition sequences per bootstrap replicate with tree bisection-reconnection branch swapping, limited to a maximum of 10000 trees) using PAUP ${ }^{*} 4.0 \mathrm{~b} 10$ (Sinauer Associates).

\subsubsection{RT-PCR}

RNA samples $(5 \mu \mathrm{g})$ were treated with DNase I (Amplification grade, Invitrogen) at $37^{\circ} \mathrm{C}$ for 15 min prior to RT-PCR. First-strand CDNA synthesis was performed using SuperScript III Reverse Transcriptase (Invitrogen) according to the manufacturer's instructions. PCR of UGT78D2, UGT78K1 (Glyma07g30190), Glyma08g07130, Glyma07g30190, Glyma07g30200, Arabidopsis $\beta$-tubulin, and G. max $\beta$-tubulin was performed with primers ULP/URP, UNSF/UNSR, 93F/93R, 86F/86R, 200F2/200R2, AtTubF/AtTubR, and GmTubF/GmTubR, respectively (Table 3.1).

\subsubsection{Recombinant protein expression in Escherichia coli}

The full-length UGT78K1 coding region fused in-frame with the $\mathrm{pET}-14 \mathrm{~b}$ vector (above) was transformed into the expression host $E$. coli $\mathrm{BL21}$ (DE3) pLysS (Novagen, ON, Canada) and a single colony was selected for production of recombinant proteins. Soluble recombinant proteins were isolated from E. coli 
BL21(DE3) pLysS following growth and induction at $16^{\circ} \mathrm{C}$, and purified by ion metal-affinity chromatography as indicated (Ford et al., 1998). To confirm the purity of the recombinant enzyme the eluted fractions were visualized on $12.5 \%$ acrylamide gel stained with $0.25 \%$ Coomassie blue. The amount of purified recombinant enzyme was determined using a protein assay kit (Bio-Rad Laboratories Inc.; http://www.bio-rad.com).

\subsubsection{Recombinant Enzyme Assays}

Initial enzyme assays (total volume $100 \mu \mathrm{l}$ ) consisted of $2 \mu \mathrm{g}$ of recombinant enzyme, $100 \mu \mathrm{M}$ of acceptor substrate and $5 \mathrm{mM}$ UDPG in assay solution (50 $\mathrm{mM}$ HEPES $\mathrm{pH} 8,14 \mathrm{mM} \beta$-mercaptoEtOH) and were incubated at $30^{\circ} \mathrm{C}$ for 30 $\min$. To study the relationship between activity and $\mathrm{pH}$, reactions were incubated in the following buffers, each at $50 \mathrm{mM}$ : MES, $\mathrm{pH}$ 5-6; Tris, $\mathrm{pH}$ 7-9; HEPES, $\mathrm{pH}$ 7-8; CAPS, pH 10. To determine the affects of temperature on enzyme activity reactions were carried out in a thermocycler at $10-55^{\circ} \mathrm{C}$. To measure for linearity of product formation, reactions were stopped at $20 \mathrm{~s}$ intervals up to $3 \mathrm{~min}$, using $5 \mathrm{mM}$ UDP-glucose with $10 \mu \mathrm{M}$ cyanidin or $100 \mu \mathrm{M}$ kaempferol.

The amount of each reaction product was determined by comparison of integrated peak areas of the glucoside (except for myricetin where the aglycone was used) to the corresponding standard curve analyzed on the same day. Standards were processed in the same manner as enzyme reactions with the exception that the recombinant enzyme was boiled prior to incubation. Standard curves were verified to be linear $(\mathrm{COC}>98)$ over the range investigated using 
Chem32 software (Agilent Technologies Inc., Montreal QC, Canada). All reaction solutions were brought to the specified assay temperature prior to the addition of the acceptor substrate and enzyme. Reactions containing anthocyanins or 3deoxyanthocyanidins were stopped by vortexing in $500 \mu \mathrm{l}$ of $15 \% \mathrm{HCO} 2 \mathrm{H}$ in $\mathrm{MeOH}$ for $20 \mathrm{~s}$. Reactions were prepared for HPLC by centrifugation $(21,000 \mathrm{~g}$ for 4 min at $4{ }^{\circ} \mathrm{C}$ ), followed by removal of the supernatant and concentrated to $100 \mu \mathrm{L}$ under nitrogen gas. All other reactions were stopped by vortexing in 500 $\mu$ l of EtOAc for $20 \mathrm{~s}$ followed by removal of the organic phase, which was then evaporated under a stream of nitrogen gas. The residue was resuspended in 100 $\mu \mathrm{l}$ of HPLC grade MeOH. All reactions were filtered through Teflon $(0.2 \mu \mathrm{m}$; Chromatographic Specialties) and $20 \mu$ injections were analyzed by HPLC-DAD.

\subsubsection{Enzyme Kinetics}

To measure the reaction velocity of the recombinant enzyme for acceptor substrates, the concentrations of cyanidin and kaempferol were varied from 3 to $100 \mu \mathrm{M}$ and from 5 to $500 \mu \mathrm{M}$, respectively, and $5 \mathrm{mM}$ UDP-glucose was used as the donor substrate. Enzyme assays were performed as described above but reactions were stopped at $3 \mathrm{~min}$ and $5 \mathrm{~min}$ to ensure linearity of data points. Lineweaver-Burk graphs were plotted for the calculation of $K_{m}$ and $V_{\max }$ values.

\subsubsection{Enzyme specificity}

The recombinant enzyme was tested for activity against anthocyaninidins (cyanidin, delphinidin, pelargonidin, petunidin, peonidin, malvidin), anthocyanins 
(3-O-glucosides of the anthocyanidins and cyanidin 3-O-galactoside), flavonols (kaempferol, quercetin, myricetin, isorhamnetin), flavonol-3-glucosides (3-Oglucosides of kaempferol, quercetin, and isorhamnetin), flavan-3-ols (+)-catechin and (-)-epicatechin, isoflavones (genistin, genistein and daidzein), a dihydroflavonol (dihydroquercetin), and a 3-deoxyanthocyanidin (apigeninidin), using $5 \mathrm{mM}$ UDP-glucose as a donor substrate. UDP-galactose $(5 \mathrm{mM})$ was also tested as a sugar donor using cyanidin and delphinidin acceptor substrates. Enzyme assays were performed as described above with either 10 or $100 \mu \mathrm{M}$ of acceptor substrate and stopped at $3 \mathrm{~min}$.

\subsubsection{Transgenic lines}

To obtain ugt78d2 lines transformed with UGT78K1, the full-length coding sequence of UGT78K1 was amplified from the pET-14b-UGT78K1 construct (described above) using primers BUF/BUR (Table 3.1) and cloned into the $B a m H I$ and Sacl sites of a modified pCAMBIA2300 vector (http://www.cambia.org) harboring the EntCUP4 constitutive promoter (Malik et al., 2002) and the NOS terminator between HindIII and EcoRI sites. Transformation of line ugt78d2 with the pCAMBIA2300-tCUP4::UGT78K1-tNOS construct was achieved using the Agrobacterium tumefaciens strain GV3101 as described (Clough and Bent, 1998). T1 seeds were selected on culture media and $50 \mu \mathrm{g} \mathrm{ml}^{-1}$ kanamycin then transferred to soil to set $\mathrm{T} 2$ seeds. Independent lines were identified to carry a single transgene copy if T2 seedlings exhibited a viable to non-viable ratio of $3: 1$ on selection media. Seedlings were visually 
screened for anthocyanin accumulation after 1 week of culture on anthocyanin gene induction media $(\mathrm{pH} 5.8)$ consisting of sucrose $(3 \%, \mathrm{w} / \mathrm{v})$, agar $(0.8 \%, \mathrm{w} / \mathrm{v})$ and $50 \mu \mathrm{g} \mathrm{ml}^{-1}$ kanamycin. Three independent lines which carried a single copy of the transgene, and which accumulated visible levels of anthocyanins, were selected for further analysis.

\subsubsection{HPLC-DAD and HPLC-ESI-MS analyses}

The HPLC system (an Agilent 1100 series; Agilent Technologies Inc., Montreal QC, Canada) was equipped with an autosampler with a $100 \mu \mathrm{L}$ loop, a quaternary pump (maximum pressure, 400 bars), a column thermostat and a diode array detector (DAD). The separations were achieved at $45^{\circ} \mathrm{C}$ on a Luna $\mathrm{C} 18(2), 4.6 \times 150 \mathrm{~mm}$, particle size $5 \mu \mathrm{m}$ fitted with a corresponding guardcolumn (Phenonenex Inc, Santa Ana, CA). The mobile phase system consisted of $5 \% \mathrm{HCO}_{2} \mathrm{H}$ in $\mathrm{HPLC}$ grade water (solvent $\mathrm{A}$ ) and $\mathrm{HPLC}$ grade $\mathrm{MeOH}$ (solvent B). The optimized elution conditions were a linear gradient of $10-100 \%$ B in 25 min with a flow rate of $1 \mathrm{ml} \mathrm{min}{ }^{-1}$, the column was washed for $10 \mathrm{~min}$ at $100 \% \mathrm{~B}$, brought back to starting mobile phase composition in $0.1 \mathrm{~min}$ and equilibrated for 5 min before next injection. The HPLC separations were monitored at 254,280 , 350,476 , and $520 \mathrm{~nm}$.

HPLC-ESI-MS analysis was done on an 3200QTRAP (ABI Sciex, Toronto, Canada). The characteristic fragments of $\mathrm{C} 3 \mathrm{G}$ and $\mathrm{K} 3 \mathrm{G}$ were detected in enhanced mass scan (EMS) mode in positive ionization. The optimal settings of the mass spectrometer were: curtain gas $\left(\mathrm{N}_{2}\right) 10 \mathrm{~L} \mathrm{~min}^{-1}$, ion spray voltage 4500 
$\mathrm{V}$, source gas (N2) $10 \mathrm{~L} \mathrm{~min}^{-1}$, declustring potential $+50 \mathrm{~V}$, entrance potential 10 $\mathrm{V}$, ionization energy $1.0 \mathrm{eV}$ and detector 2200.

\subsubsection{Metabolite Analysis}

One-week-old Arabidopsis seedlings $(100 \mathrm{mg})$ cultured on anthocyanin gene induction media (above) were pulverized in $200 \mu \mathrm{L}$ of $80 \% \mathrm{MeOH}$ in water using a FastPrep FP120 Homogenizer (Savant). The extracts were filtered through Teflon ( $0.2 \mu \mathrm{m}$; Chromatographic Specialties), $20 \mu$ injections were analyzed by HPLC-DAD as described above, and $5 \mu \mathrm{l}$ were measured by photospectroscopy using a NANODROP 2000 (Thermo Scientific) the formula $A_{530}-0.25 A_{657}$ to compensate for chlorophyll absorption at $530 \mathrm{~nm}$ (Mancinelli, 1990).

\subsection{Results}

3.3.1. Cloning and analysis of a glycosyltransferase gene from the seed coat of black soybean

As the Glyma1 soybean genome sequence was not available at the time this study was initiated, Glycine max ESTs from the GenBank collection were searched with the tBLASTn and BLASTn algorithms using every published UF3GT sequence as a query (September, 2007), including the only published legume UF3GT (UGT78G1, from Medicago truncatula) (Modolo et al., 2007) in order to identify UF3GT candidates. However, these searches failed to identify any sequences with exceptionally high similarity. Highest similarity to GenBank 
G. max ESTs (up to $53 \%$ ) was identified by tBLASTn using the PSPG box motifs of UGT78D2 from Arabidopsis (Tohge et al., 2005) and VvGT1 from Vitis vinifera (Ford et al., 1998). However, these searches yielded numerous unigenes with moderate similarity, and thus did not provide a sufficiently narrow pool of candidates for functional analysis. The GenBank database was searched using keywords such as anthocyanidin 3-O-glucosyltransferase which yielded the Ct3GT-A sequence (AB185904), representing a UF3GT from the legume Clitoria ternatea ( $\mathrm{N}$. Noda, personal communication). A megablast search using the Ct3GT-A mRNA as a query identified five ESTs with exceptionally high identity (68-81\%). The ESTs were derived from various organs including seeds and flowers.

RNA was isolated from the seed coats of black soybean (G. max cv. Clark) and the three ESTs with highest identity to Ct3GT-A were extended by 5'and 3'-RACE and sequenced. RACE fragments from the three ESTs were found to encode a single contiguous mRNA (1,661 nts in length). The 5'- and 3'untranslated regions were $86 \mathrm{bp}$ and $234 \mathrm{bp}$, respectively. Conceptual translation of the mRNA revealed a 447-amino acid protein, which we named UGT78K1. The presence of the plant secondary product glucosyltransferase (PSPG) consensus sequence in the C-terminal region of UGT78K1 identified it to be a type I glycosyltransferase (Vogt and Jones, 2000).

3.3.2. Genomic and phylogenetic analyses of soybean glycosyltransferases 
To examine the potential for gene redundancy, a search for soybean genes with high identity to UGT78K1 (Glyma07g30180) was performed. A BLASTn search of the soybean (Glyma1) genome sequence (www.phytozome.net/soybean) revealed four sequences (Glyma07g30200, Glyma06g39350, Glyma07g30190, and Glyma08g07130) with high identity to UGT78K1 (83, 85, 86, and 93\% respectively). Glyma08g07130, Glyma07g30190, and Glyma07g30200 had high amino acid identity $(93,79$, and $72 \%$, respectively) to UGT78K1, however conceptual translation of Glyma06g39350 revealed a highly truncated protein of 265 amino acids with two $\mathrm{N}$-terminal deletions and the absence of the putative catalytic histidine residue equivalent to His20 of VvGT1 (Offen et al., 2006). This suggested that Glyma06g39350 may be non-functional, and thus it was excluded from further analysis.

Figure 3.1 demonstrates the genomic organizations of UGT78K1 and the Arabidopsis gene UGT78D2 with their respective paralogs. Interestingly, the UGT78K1 genomic sequence is positioned in the same orientation and situated directly upstream from Glyma07g30190 and Glyma07g30200 (Figure 3.1A), with a fourth highly similar sequence (Glyma08g07130) located on a separate chromosome (not shown). A similar genomic organization has been reported for UGT78D2 (At5g17050, ANL1) and three paralogs (At5g17030, At5g17040, and At1g30530) in the Arabidopsis genome (Figure 3.1B) (Kubo et al., 2007). 


\section{A}

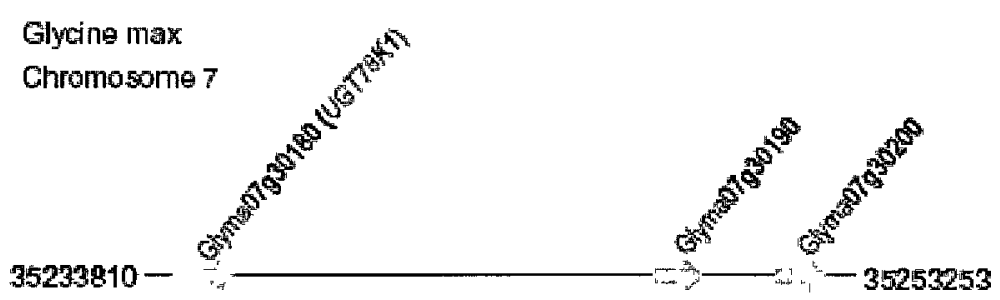

B

Ar abidopsis the liarea

Chromasome 5

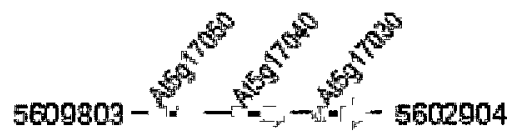

$1 \mathrm{~kb}$

Figure 3.1. Genomic organisations of UGT78K1 (Glyma07g30180), Glyma08g07130 and Glyma07g30190 from Glycine max (A) and Arabidopsis thaliana glycosyltransferases UGT78D2 (At5g17050), At5g17040, and At5g17030 (B). 
However, unlike the Arabidopsis sequences, UGT78K1 and its paralogous sequences lack the highly conserved glycosyltransferase (UGT) intron, equivalent to the Arabidopsis UGT intron 2 (Li et al., 2001). This data suggested that the gene triplication event that gave rise to UGT78K1 and its paralogs on chromosome 7 (Glyma07g30190 and Glyma07g30200) most likely took place after divergence of G. max and Arabidopsis from a common ancestor, and thus the similar genomic organizations likely do not represent synteny. Furthermore, as Glyma08g07130 has greatest similarity to UGT78K1, this suggests that the gene duplication event that gave rise to this sequence occurred more recently than the triplication event that resulted in UGT78K1, Glyma07g30190, and Glyma07g30200 on chromosome 7.

Figure 3.2 demonstrates a phylogenetic analysis of the amino acid sequences of biochemically characterized flavonoid UGTs. The in vitro substrate specificities and in vivo functions of flavonoid UGTs cannot be accurately predicted using amino acid sequences alone. However, phylogenetic analysis resulted in five clusters that can be generally grouped based on common in vitro substrate specificities. Enzymes in Cluster $\mathrm{V}$ have anthocyanin 5-O- and/or flavonol 7-OUGT activities, Cluster IV glycosylates flavonol and isoflavone substrates, and Cluster III includes isoflavone 7-O- and anthocyanidin 3,5-O-GT activities. Cluster II GTs have multiple substrate specificities generally for chalcones, flavones, and flavonols, but not anthocyanidins. Cluster I is exclusively UF3GTs. UGT78K1 groups with Cluster I, as does its paralogs (Glyma08g07130, Glyma07g30190, and Glyma07g30200). UGT78K1 groups more closely with its 


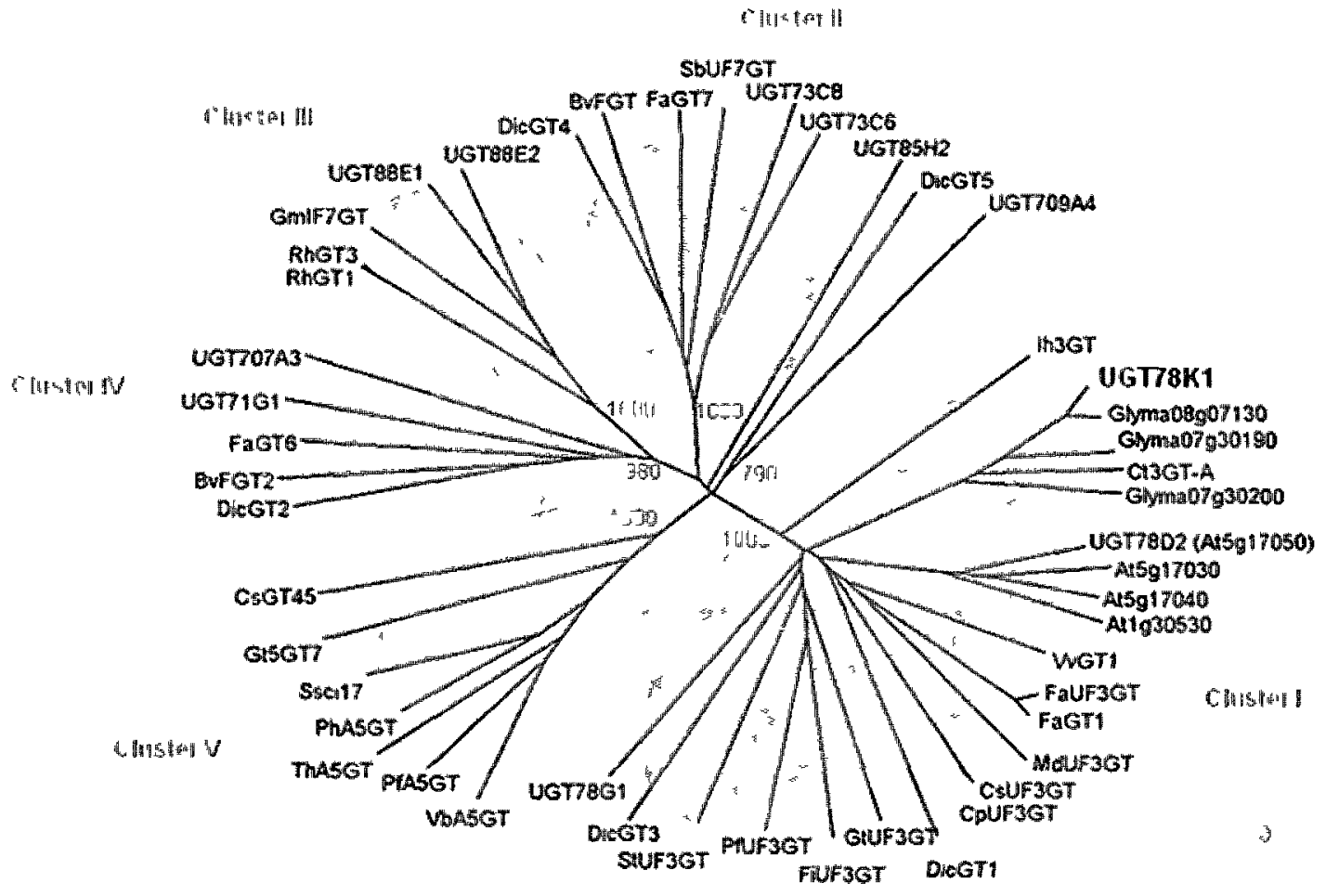

Figure 3.2. Unrooted phylogenetic tree of UGT78K1 and flavonoid glycosyltransferase amino acid sequences. GenBank accessions: UGT78K1 (GU434274; Phytozome landmark: Glyma07g30180), Glyma07g30190 (Phytozome landmark: Glyma07g30190), Glyma07g30200 (Phytozome landmark: Glyma07g30200), Glyma08g07130 (Phytozome landmark: Glyma08g07130), Ct3GT-A (AB185904), UGT78D2 (NM_121711), At5g17030 (NC_003076), At5g17040 (NC_003076), At1g30530 (NM_102790), VhA5GT (AB013598), PfUA5GT (AB013596), GtUF3GT (D85186), VvGT1 (AF000372), Ih3GT (AB161175), DicGT1 (AB191245), DicGT2 (AB191246), DicGT3 (AB191247), DicGT4 (AB191248), DicGT5 (AB191249), RhGT1 (AB201048), RhGT2 (AB201049), RhGT3 (AB201050), GmIF7GT (AB292164), Ssci17 (AY033489), FaGT1 (AY663784), FaGT6 (DQ289587), FaGT7 (DQ289588), UGT73C8 (DQ875459), UGT88E1 (DQ875460), UGT88E2 (DQ875461), UGT71G1 (AAW56092), UGT85H2 (DQ875463), UGT78G1 (DQ875464), CsUF3GT (AY519364), FaUF3GT (AY695815), FiUF3GT (AF127218), MdUF3GT (DQ156906), PfUF3GT (AB002818), StUF3GT (AY954034), CpUF3GT (GQ141630), SbUF7GT (AB031274), BvFGT (AY526080), BvFGT2 (AY526081), UGT73C6 (NM_129234), UGT707A3 (BAC83989), UGT709A4 (BAC80066), Gt5GT7 (AB363839), CsGT45 (FJ194947). The number adjacent branches indicate maximum parsimony bootstrap values for the corresponding node. The scale bar indicates the number of differences per 100 residues derived from the ClustalW alignment. The phylogenetic tree was generated using TreeView software (Page 1996). 
paralogs located on chromosome 7 (Figure 3.1A) separately from UGT78D2 and its paralogs (Figure 3.2). This further suggests that the gene triplication event that resulted in similar genomic organizations for UGT78K1 and UGT78D2, and their paralogs (Figure 3.1), likely occurred after speciation and is not a result of synteny.

\subsubsection{Expression analysis of black soybean glycosyltransferases}

To determine whether the expression of UGT78K1 coincided spatially with anthocyanin and/or flavonol biosynthesis in black soybean, RT-PCR was performed on RNA isolated from individual organs (Figure 3.3). Anthocyanins accumulate to visible levels in the seed coat, mature stem, and purple flower petals of black soybean cv. Clark. By contrast, flavonol glycosides have been reported to accumulate in all aerial organs of soybean (Graham, 1991). RT-PCR identified UGT78K1 transcripts in the seed coat, flower bud, stem, and all other organs examined (Figure 3.3). These results suggest that UGT78K1 is expressed not only at the sites of anthocyanin biosynthesis, but also at sites of flavonol glycoside biosynthesis. A similar expression pattern was observed for UGT78D2 (ANL1) in Arabidopsis (Kubo et al., 2007). RT-PCR demonstrated overlapping expression profiles of Glyma07g30200, Glyma07g30190, and Glyma08g07130 with UGT78K1 in some organs, but only Glyma08g07130 was co-expressed with UF3GT in the seed coat (Figure 3.3). 


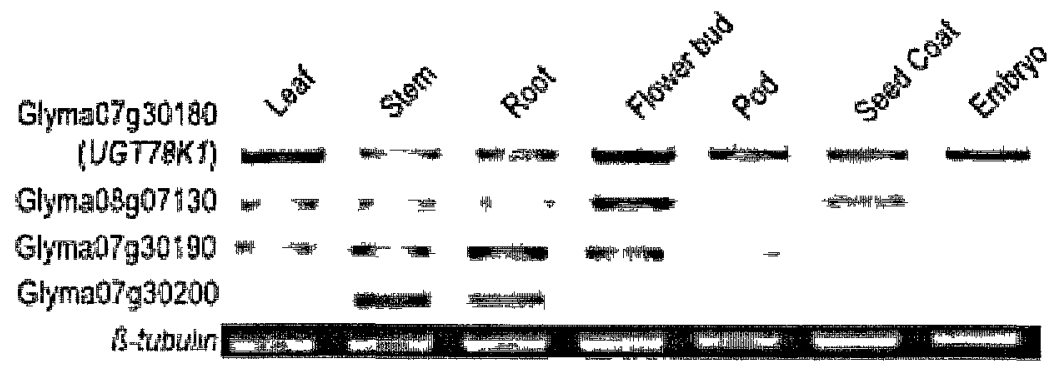

Figure 3.3. Transcript detection of UGT78K1 (Glyma07g30180), Glyma08g07130, Glyma07g30190, and Glyma07g30200 by RT-PCR with genespecific primers. 
3.3.4. UGT78K1 encodes a UF3GT with strict specificity for anthocyanidins and flavonols in vitro

The open reading frame of UGT78K1 was cloned into the pET-14b N-terminal hexahistidine fusion tag expression vector (Novagen, ON, Canada), transformed into BL21(DE3) pLysS Escherichia coli cells, and expressed at $16^{\circ} \mathrm{C}$ for $24 \mathrm{~h}$. Protein was extracted and his-tagged UGT78K1 (rUGT78K1) was purified from the soluble fraction by ion-metal affinity chromatography (IMAC) using the Talon system (Clontech, Palo Alto, CA). Purified fractions obtained from a $250 \mathrm{ml} E$. coli culture contained $1.6-2.4 \mathrm{mg}$ of the fusion protein. Separation of the IMAC fractions by SDS-PAGE followed by staining with Coomassie Blue revealed an apparently pure recombinant enzyme with an estimated molecular mass of ca $49.8 \mathrm{kDa}$ (Figure 3.4A). This value matched well with the calculated molecular mass of UGT78K1 (49.24 kDa) with the inclusion of its $2.18 \mathrm{kDa}$ N-terminal tag. Preliminary assays using uridine diphosphate glucose (UDPG) as a sugar donor demonstrated that the purified rUGT78K1 could transfer glucose to the 3-position of the anthocyanidin cyanidin (Figure 3.4B, D) and the flavonol kaempferol (Figure 3.4C, E), whereas the boiled enzyme and bacteria expressing the corresponding empty vector could not catalyze these reactions (not shown). Glycosylated products from the recombinant enzyme assays were identified and quantified by high performance liquid chromatography-diode array detection (HPLC-DAD) by comparison to authentic standards. The presence of cyanidin-3O-glucoside (C3G) and kaempferol-3-O-glucoside (K3G) reaction products were verified by high performance liquid chromatography electrospray ionization mass 
A

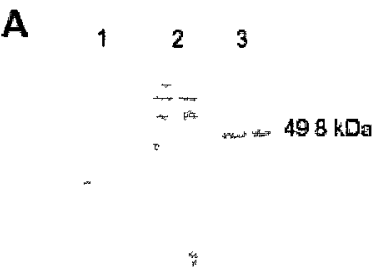

C

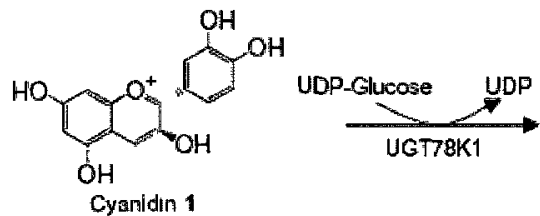<smiles>Oc1ccc([C@H]2Oc3c(O)cc(O)cc3[C@H](O)[C@H]2O)cc1</smiles>

D

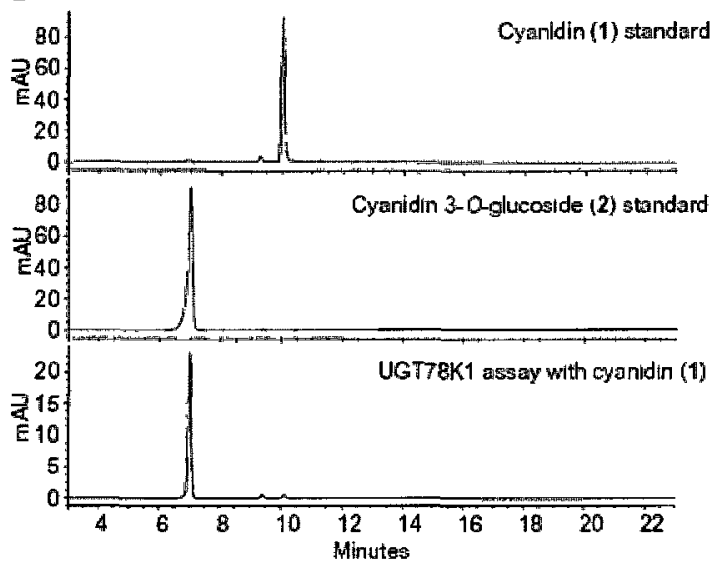

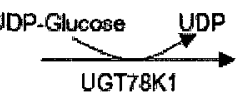

$E$

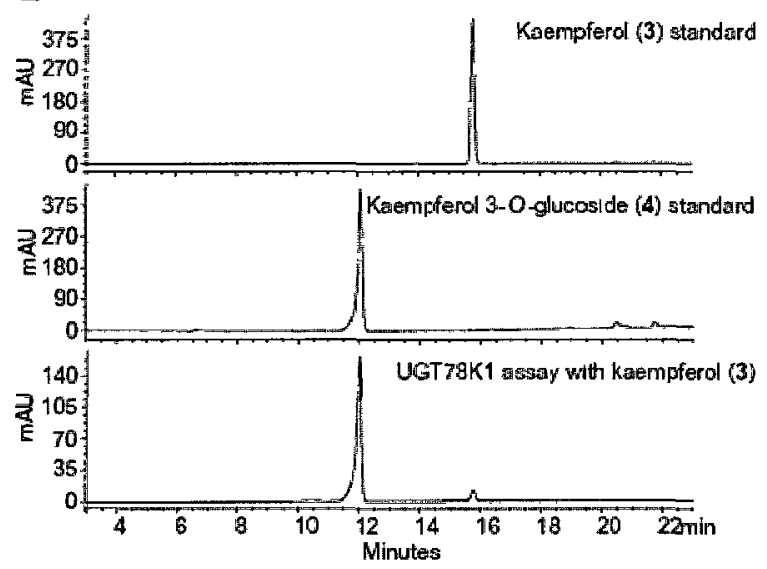

Figure 3.4. Purification of his-tagged UGT78K1 and identification of reaction products. (A) SDS-PAGE analysis of UGT78K1: total soluble protein from E. coli expressing UGT78K1 prior to induction with $100 \mu \mathrm{M} \mathrm{IPTG}$ (lane 1), $24 \mathrm{~h}$ postinduction (lane 2); purified UGT78K1 (lane 3). (B, C) UGT78K1 enzyme reactions as revealed by HPLC chromatograms at $520 \mathrm{~nm}(D)$ and $350 \mathrm{~nm}(\mathrm{E})$, respectively. HPLC retention times: cyanidin (Rt: 10.0 min); cyanidin 3-Oglucoside (Rt: 7.0); kaempferol (Rt: 15.8); kaempferol 3-O-glucoside (Rt: 12.0). 
spectrometry (HPLC-ESI-MS) in enhanced mass scan mode. The characteristic fragments detected in the $\mathrm{C} 3 \mathrm{G}$ reaction mixture were 287.6 [cyanidin $+\mathrm{H}^{+1}$ and 450.1 [cyanidin-3-O-glucoside $+\mathrm{H}]^{+1}$, and 287.1 [kaempferol $\left.+\mathrm{H}\right]^{+1}$ and 449.1 [kaempferol-3-O-glucoside $+\mathrm{H}]^{+1}$ for the $\mathrm{K} 3 \mathrm{G}$ reaction mixture. Further purification of the enzyme by the removal of its hexahistidine tag did not alter enzyme activity, as determined by measuring activity before and after cleavage. For this reason, assays were routinely performed without removal of the tag. The enzyme exhibited no significant loss in activity towards kaempferol after storage at $4{ }^{\circ} \mathrm{C}$ or $-20^{\circ} \mathrm{C}$ in a $50 \%$ glycerol solution for $24 \mathrm{~h}$, however longer incubations resulted in a notable reduction in activity (not shown). To minimize the effect of storage on enzyme activity, assays were performed immediately following purification.

Optimal reaction conditions were determined using cyanidin and kaempferol as acceptor substrates with UDPG as the donor because cyanidin 3-O-glucoside and kaempferol glycosides are the most abundant anthocyanins and flavonols in the seed coats and purple petals of G. max, respectively (Choung et al., 2001; Iwashina et al., 2007; Lee et al., 2009). Investigation of a pH range from 5 to 10 and temperatures from 10 to $50^{\circ} \mathrm{C}$ revealed the recombinant enzyme to be most active at $\mathrm{pH} 8$ and $30^{\circ} \mathrm{C}$. rUGT78K1 exhibited a slight buffer preference for HEPES over Tris at pH 8 (not shown).

The kinetics of rUGT78K1 was determined for both cyanidin and kaempferol acceptor substrates (Figure 3.5). By using a concentration of $5 \mathrm{mM}$ UDPG and varying the concentration of kaempferol between 5 and $400 \mu \mathrm{M}$, a hyperbolic saturation curve was obtained (Figure 3.5A), from which Lineweaver-Burk 
transformation gave an apparent $K_{m}$ for kaempferol of $174 \mu \mathrm{M}$ and $V_{\max }$ of 24.8 pKat $\mu \mathrm{g}^{-1}$. From these a $k_{\text {cat }}$ of $1.25 \mathrm{~s}^{-1}$ was calculated. By contrast, when the concentration of UDPG was maintained at $5 \mathrm{mM}$ and the concentration of cyanidin was varied from 5 to $100 \mu \mathrm{M}$, the reaction velocity increased with cyanidin concentrations up to circa $16 \mu \mathrm{M}$ and progressively decreased with increasing cyanidin concentrations (Figure 3.5B), suggesting the enzyme was inhibited by this substrate.

The recombinant enzyme readily transferred glucose from UDPG to 3-position of all tested anthocyanidins and flavonol aglycones with variable specificities (Figure 3.6). When acceptor substrates were supplied below the inhibitory concentration of cyanidin the enzyme demonstrated highest activity towards the anthocyanidin delphinidin (Figure 3.6A). By contrast, when acceptor substrates were supplied above the inhibitory concentration of cyanidin, UGT78K1 demonstrated highest activity for flavonols (quercetin, myricetin, and kaempferol) (Figure 3.6B, white bars). No activity was observed for other flavonoid classes previously identified in soybean, including dihydroflavonol (dihydroquercetin), isoflavone (genistin, genistein and daidzein), (epi)-flavan-3-ol (catechin, epicatechin), 3-deoxyanthocyanidin (apigeninidin), flavonol conjugate (flavonol 3O-glucosides) and anthocyanins (anthocyanidin 3-O-glucosides and cyanidin 3O-galactoside) (data not shown). However, galactose could be transferred from UDP-galactose to the 3-position of cyanidin and delphinidin with low specificity relative to the analogous glucosyltransferase reactions (Figure 3.6, grey bars), as determined by HPLC-DAD retention times and absorbance spectra by 

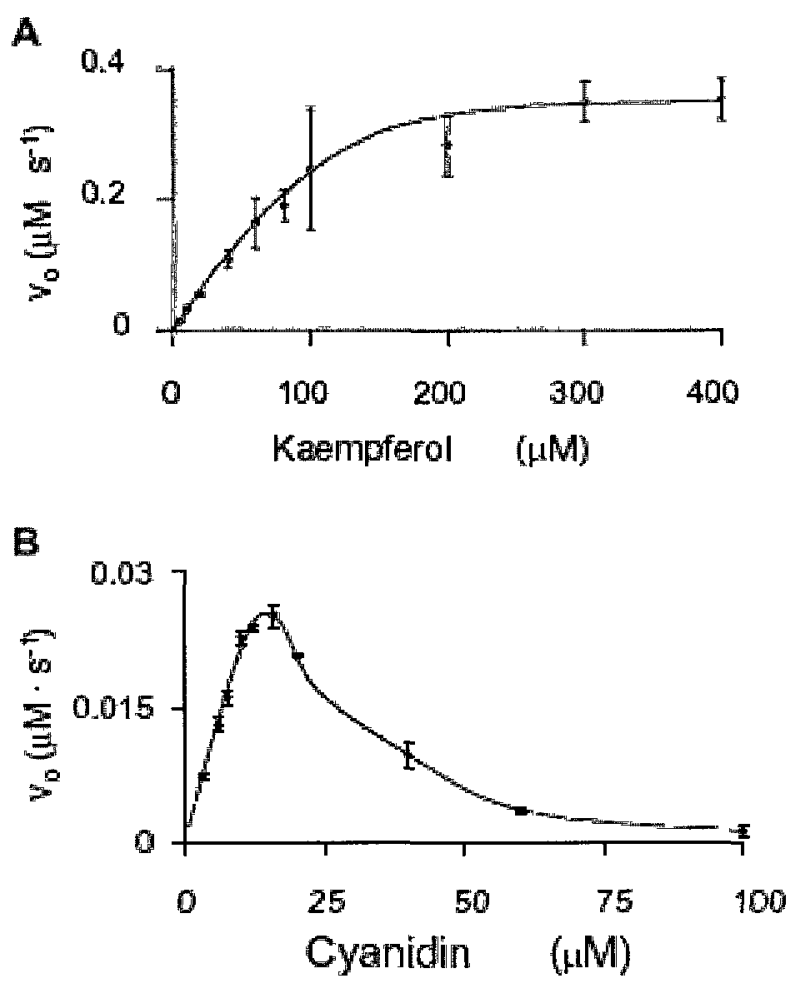

Figure 3.5. UGT78K1 kinetics for cyanidin and kaempferol acceptor substrates. Reaction kinetics for acceptor substrates kaempferol (A) and cyanidin (B) using 5 $\mathrm{mM}$ UDPG as a sugar donor. Points represent the mean of three assays \pm the standard deviation. 
A
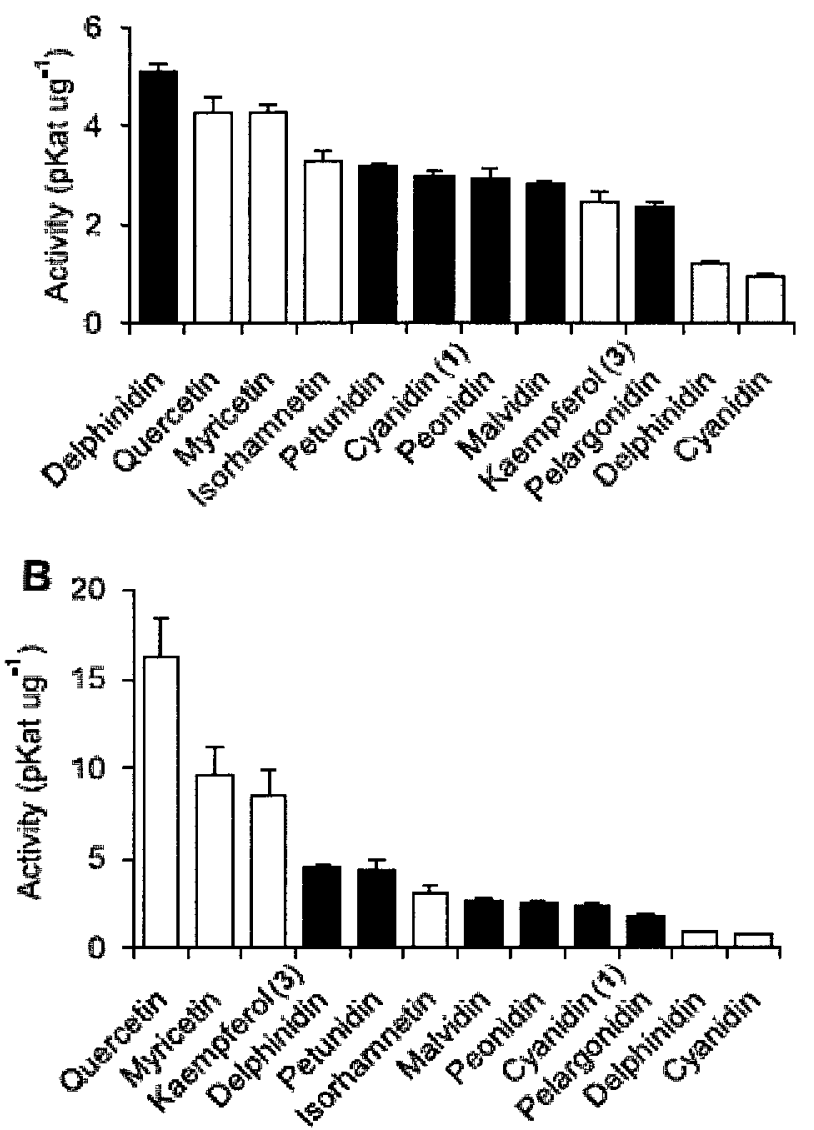

Figure 3.6. Activities of UGT78K1 for anthocyanidins and flavonols. (A) Acceptor substrate concentration $10 \mu \mathrm{M}$. (B) Acceptor substrate concentration $100 \mu \mathrm{M}$. Anthocyanidins (black bars) and flavonols (white bars) assayed using $5 \mathrm{mM}$ UDPG as a sugar donor. Cyanidin and delphinidin assayed using $5 \mathrm{mM}$ UDPgalactose as a donor (grey bars). Each bar represents the mean of three assays + the standard deviation. 
comparison to authentic standards. These results strongly suggest that rUGT78K1 acts exclusively on anthocyanidins and flavonols in vitro preferentially as a glucosyltransferase with strict regiospecificity for the 3-position.

3.3.5. UGT78K1 functions as a 3-O-glucosyltransferase in anthocyanin and flavonol biosynthesis in vivo

To confirm that UGT78K1 has flavonoid 3-O-glucosyltransferase activity in vivo, the cDNA was transferred into the Arabidopsis mutant ugt78d2 with highly reduced anthocyanidin and flavonol 3-O-glucosyltransferase activity (Tohge et al., 2005). T1 seedlings were selected on media containing kanamycin, transferred to soil and grown to maturity. Thirty percent of mature T1 plants (12 independent lines) selected on kanamycin media exhibited pigmentation at the rosette base similar to the wild type, whereas the ugt78d2 mutant transformed with the empty vector lacked visible pigments (not shown). Three lines (U4-8, U425 , and U4-28) were identified that carry a single copy of the UGT78K1 CDNA by selection of $T 2$ seedlings on kanamycin media $\left(x^{2} 0_{05,1}(3: 1\right.$, viable:non-viable $)=$ $1.7,0.1$, and 0.005 , respectively) and were chosen for further analysis. Presence of the UGT78K1 transgene and T-DNA insertions in the UGT78D2 gene were verified by PCR using primers UHF/UHR and LB/URP, respectively, whereas the UGT78D2 was indentified to lack the T-DNA insertion using primers ULP/URP (Figure 3.7A). RT-PCR was used to verify that each transgenic line expressed UGT78K1 and lacked the expression of UGT78D2 (Figure 3.7B). 
To examine anthocyanin pigmentation phenotype more clearly, T2 seedlings were cultured on anthocyanin induction media (see Materials and methods) and were visualized using light microscopy. The majority of T2 seedlings displayed highly pigmented cotyledons and hypocotyls similar to the wild type, whereas the ugt78d2 mutant transformed with the empty vector had minimal amounts of visible pigments (Figure 3.7C). To examine the amounts of total anthocyanins in transgenic lines relative to wild type and ugt78d2 controls, $100 \mathrm{mg}$ of seedlings from each line were pooled, extracted with $80 \% \mathrm{MeOH}$, and total anthocyanins were quantified spectrophotmetrically using a formula to compensate for chlorophyll absorption. On average, extracts from transgenic lines contained five (line U4-8) to six times (line U4-28) the anthocyanin absorbance relative to the ugt78d2 mutant (Figure 3.7D) and 65\% (U4-8) to 75\% (U4-28) absorbance relative to the wild type. This suggested restoration of anthocyanin biosynthesis in transformed seedlings, as $25 \%$ of the segregating T2s on non-selective anthocyanin induction media would not carry the UGT78K1 gene. 
A

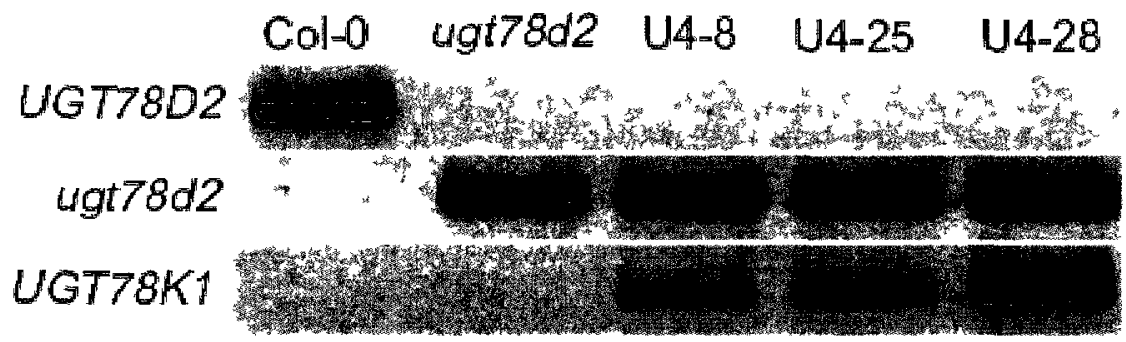

B

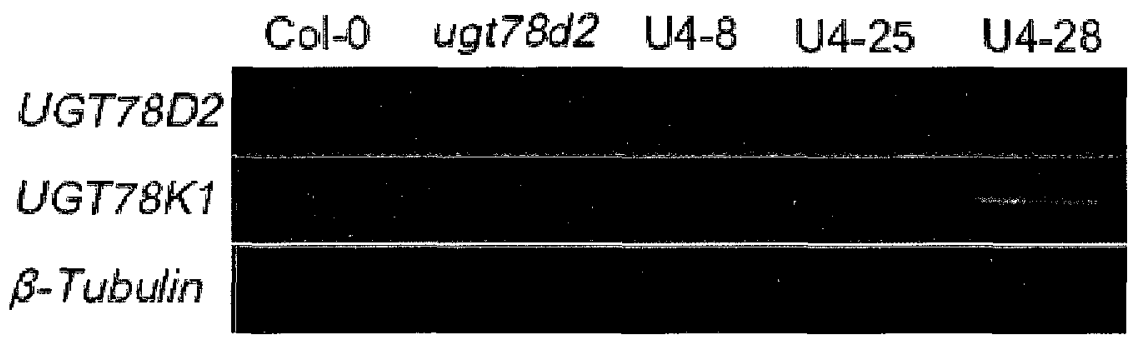

C

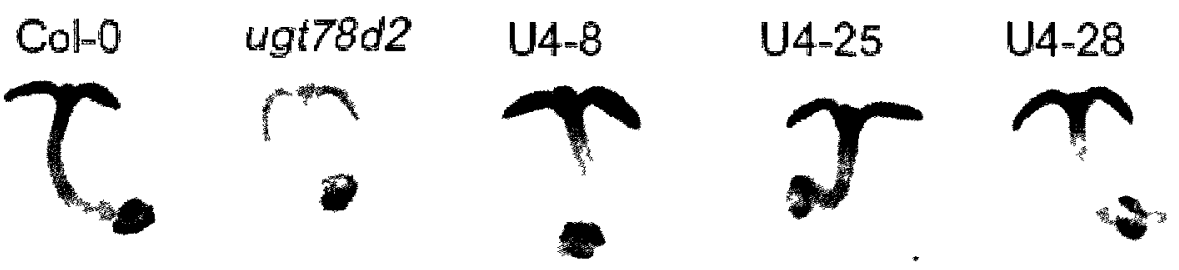

D

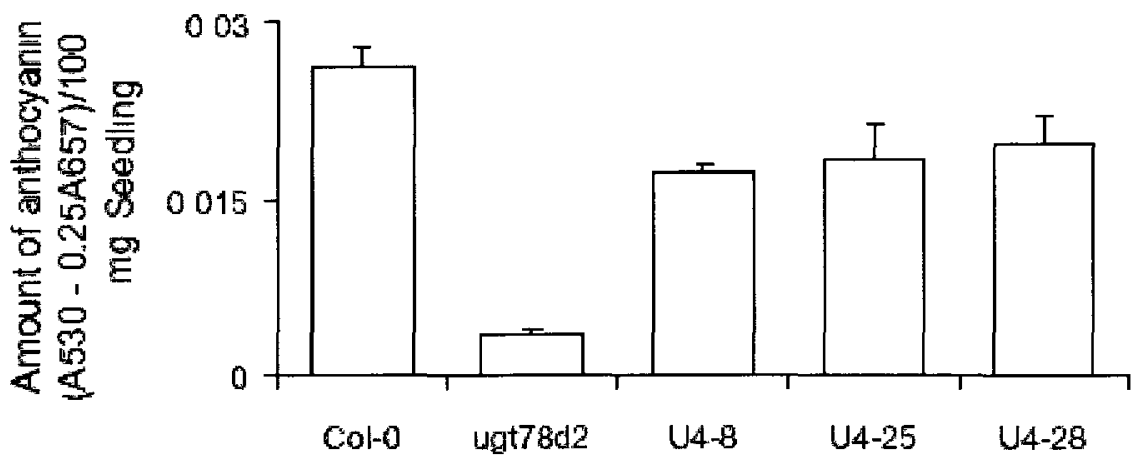

Figure 3.7. The UGT78K1 gene complements anthocyanın biosynthesis in the Arabidopsis ugt78d2 mutant. (A) PCR analysis for the UGT78K1 and UGT78D2 genes and the T-DNA-inserted allele ugt78d2 from genomic DNA of wild type (Columbia), mutant ugt78d2 (transformed with empty pCAMBIA1300tCUP plasmid), and the ugt78d2 mutant transformed with the UGT78K1 gene (three 
independent T2 lines; U4-8, U4-25, U4-28) . (B) RT-PCR analysis of total RNA from $100 \mathrm{mg}$ of pooled seedlings from each line. (C) T2 lines cultured on anthocyanin induction media for 1 week (see materials and methods). (D) Amount of anthocyanins in $100 \mathrm{mg}$ of pooled T2 seedlings cultured on anthocyanin induction media.

To determine the relative amounts of individual anthocyanins and flavonols in transgenic lines relative to the wildtype and $u g t 78 d 2$ controls, $100 \mathrm{mg}$ of seedlings (T2s) from each line were pooled, extracted with $80 \% \mathrm{MeOH}$, and analyzed by HPLC-DAD. The ugt78d2 mutant transformed with the empty vector had reduced peak area for all peaks measured at $520 \mathrm{~nm}$ (anthocyanins) and some peaks measured at $350 \mathrm{~nm}$ (flavonoids) relative to the wildtype (Figure 3.8). Highly similar profiles were reported previously for Col-0 and the ugt78d2 mutant, and the peaks reduced at $520 \mathrm{~nm}$ and $350 \mathrm{~nm}$ in the ugt78d2 mutant relative to the wildtype were identified to be anthocyanins and flavonols, respectively, that contained a glucose at the 3-position (Tohge et al., 2005). The pooled T2 seedlings expressing the UGT78K1 cDNA in the ugt78d2 background had restored peaks, albeit to lower levels than the wild type. These results strongly suggest that UGT78K1 has similar activity to UGT78D2, a flavonoid 3-Oglucosyltransferase involved in anthocyanin and flavonol biosynthesis in vivo. 

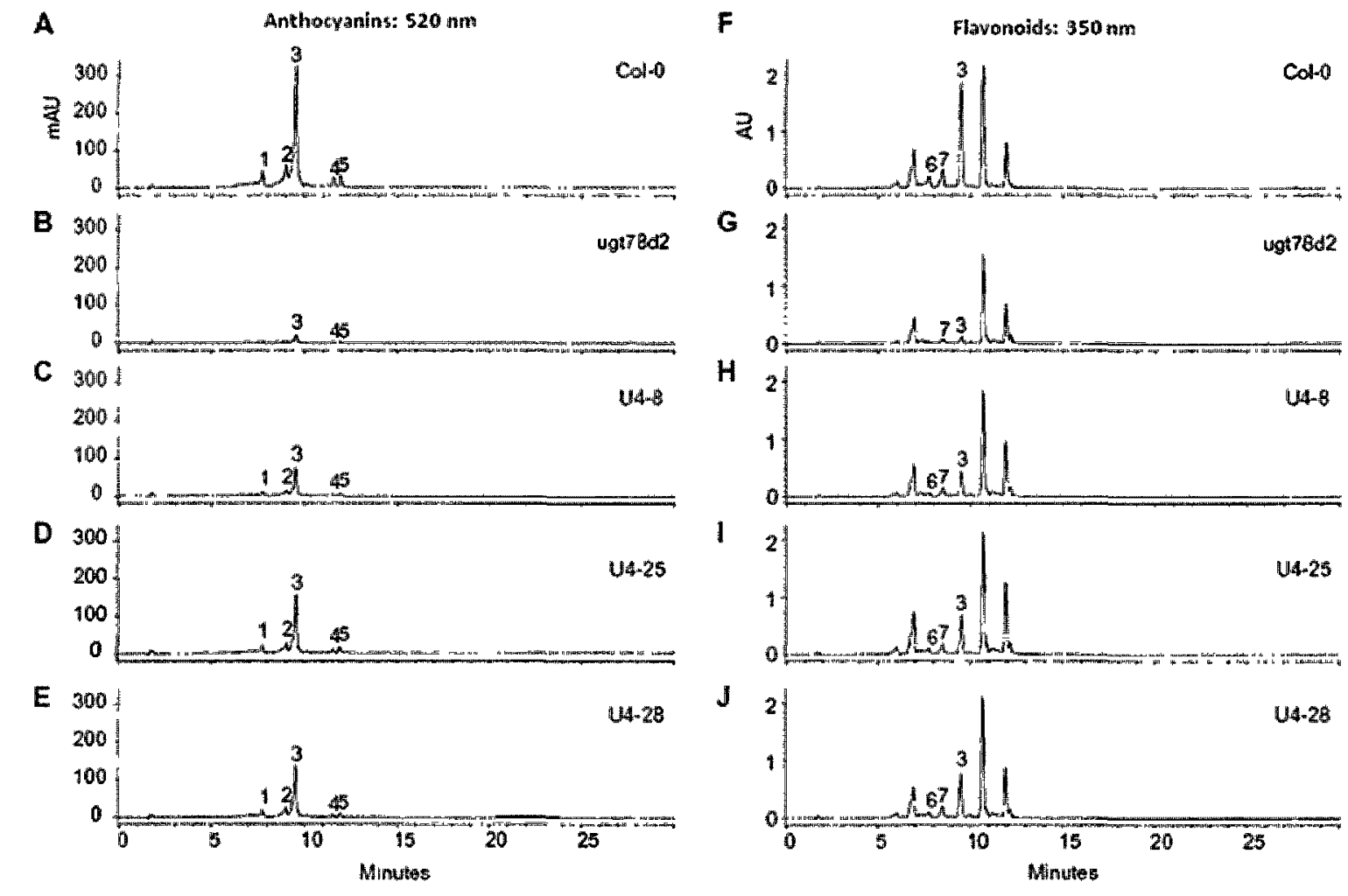

Figure 3.8. Functional analysis of the UGT78K1 gene by complementation of the Arabidopsis ugt78d2 null mutation. (A-J) HPLC chromatograms of $80 \%$ aq.

$\mathrm{MeOH}$ extracts of seedlings cultured on anthocyanin induction media for 1 week. (A, F) Col-0, (B, G) ugt78d2, (C, H) U4-8, (D, I) U4-25, (E, J) U4-28. (A-E) Absorbance at $520 \mathrm{~nm}$ for analysis of anthocyanins. (F-J) Absorbance at $350 \mathrm{~nm}$ for analysis of flavonoids. HPLC retention times: peak 1 (Rt: 7.8); peak $2(R \mathrm{t}$ :

9.0); peak 3 (Rt: 9.6); peak 4 (Rt: 11.6); peak 5 (Rt: 11.9); peak 6 (Rt: 7.7); peak 7 (Rt: 8.5). 


\subsection{Discussion}

Several genes involved in common flavonoid biosynthesis steps have been characterized from black soybean, however the gene catalyzing the final step in anthocyanin biosynthesis, namely UDP-glucose:flavonoid 3-O-glucosyltransferase (UF3GT), remained unreported (reviewed by Kovinich et al., 2011a). The purpose of this study was two-fold: 1) to identify the gene catalyzing the final step in anthocyanin biosynthesis from the seed coat of black soybean to provide a possibility of engineering reduced pigment in transgenic grains, and 2) to characterize the catalytic properties of the recombinant enzyme to provide a basis for future glucosyltransferase structure-function analyses.

The first UGT gene isolated from plants was the UDP-glucose:flavonol glucosyltransferase from Zea mays, encoded by the bronze locus (Fedoroff et al., 1984). Since then, UF3GT enzymes have been partially purified from Brassica oleracea (Saleh et al., 1976), Haplopappus gracilis (Saleh et al., 1976), Senecio $x$ hybridus (Ogata et al., 1998), and cDNAs have been cloned into expression vectors for characterization of recombinant UF3GT activities from Gentiana triflora (Tanaka et al., 1996), Vitis vinifera (Ford et al., 1998), Dianthus caryophyllus (Ogata et al., 2004), Iris hollandica (Yoshihara et al., 2005), Arabidopsis (Tohge et al., 2005), Fragaria $x$ ananassa (Almeida et al., 2007), Medicago truncatula (Modolo et al., 2007; Peel et al., 2009) and Citrus paradisi (Owens and Mclntosh, 2009). Homology-based searches of the GenBank database using each reported UF3GT as a query identified numerous soybean 
unigenes with equally low similarity. This suggested that soybean UF3GT(s) and other putative glycosyltransferases are highly divergent from published UF3GTs, including the only previously reported legume UF3GT UGT78G1 from Medicago truncatula (Peel et al., 2009).

All recombinant UF3GTs characterized to date have unique substrate specificities in vitro. Generally, some prefer anthocyanidins (Tanaka et al., 1996; Ford et al., 1998; Ogata et al., 2004; Yoshihara et al., 2005; Almeida et al., 2007), while others prefer flavonols or (iso)flavonoids (Modolo et al., 2007; Owens and Mclntosh, 2009). UGT78K1 glucosylated only anthocyanidins and flavonols, however its relative substrate preference was dependent on the concentration of acceptor substrate (Figure 3.6). Kinetic analyses suggested that the enzyme is inhibited by cyanidin at higher concentrations (above circa $16 \mu \mathrm{M}$ ) (Figure 3.5B). The fact that high amounts of cyanidin remained after enzyme reactions (not shown) supports the notion of substrate inhibition rather than degradation of cyanidin as the cause of the observed kinetics. Future studies will include full kinetics analyses for all 10 acceptor substrates to determine whether they are similarly inhibiting UGT78K1 activity, and kinetics assays with an acceptor substrate (e.g. kaempferol) and an inhibitor (cyanidin) to gain more insight into the underlying mechanism of UGT78K1 inhibition.

UF3GTs from red cabbage (B. oleracea) and H. gracilis (Saleh et al., 1976; Saleh et al., 1976) have also been shown to be inhibited by cyanidin. However, it remains unclear whether inhibition of UF3GT activity by cyanidin could have a 
role in the regulation of flavonoid biosynthesis in vivo. The possibility exists that inhibition of UGT78K1 by cyanidin could prevent the cytotoxic effects of high cytosolic cyanidin 3-O-glucoside concentrations as observed in glutathione Stransferease mutants defective for vacuolar sequestration of anthocyanins (Marrs et al., 1995; Alfenito et al., 1998). Alternatively, inhibition of UGT78K1 by cyanidin 3-O-glucoside could function to make available more anthocyanidin substrates for proanthocyanidin biosynthesis, as anthocyanidins are precursors of both pathways (Lepiniec et al., 2006), and anthocyanins and PAs coaccumulate in palisade cells of black soybean during seed development (Kovinich, unpublished data). However, another possibility is that localized cyanidin concentrations are not high enough in soybean to cause the substrate inhibition of UGT78K1 that is observed in vitro. Flavonoid enzymes are thought to physically associate in metabolons (Saslowsky and Winkel-Shirley, 2001; Winkel, 2009). Unfortunately, it is presently not possible to measure the localized concentrations of flavonoid intermediates within metabolons to determine whether these concentrations are comparable to those which are observed to cause inhibiton in vitro.

Transfer of UGT78K1 into the Arabidopsis mutant ugt78d2 restored both anthocyanin and flavonol glycoside biosyntheses (Figure 3.8), confirming the in vivo function of UGT78K1 as a UF3GT. Anthocyanin levels were not restored to a lesser degree than flavonols in Arabidopsis despite the ability of cyanidin to inhibit UGT78K1 in vitro (Figure 3.5B). This suggested that substrate inhibition by cyanidin was not operating in vivo and may have been a result of excessive 
amounts of the UGT78K1 enzyme relative to the amount of cyanidin substrate. Similar to UGT78K1, the M. truncatula UF3GT UGT78G1 exhibited relatively low activity towards cyanidin compared to other flavonoid substrates in vitro (Modolo et al., 2007) , but was demonstrated to act in anthocyanin biosynthesis in vivo (Peel et al., 2009). These results raise the possibility that in vitro specificities may better reflect UF3GT capability rather than major in vivo function(s).

The $V_{\max }$ value of UGT78K1 for kaempferol $\left(24.8 \mathrm{nKat} \mu \mathrm{g}^{-1}\right)$ was slightly higher than those reported previously for UF3GTs and flavonol substrates (11.6 to $20.5 \mathrm{nKat}_{\mu g^{-1}}$ ) (Ford et al., 1998; Owens and Mclntosh, 2009). The $K_{m}$ values for flavonoid substrates from characterized UF3GTs vary greatly among species with values ranging from $1.5 \mu \mathrm{M}$ to $400 \mu \mathrm{M}$ (Saleh et al., 1976; Saleh et al., 1976; Ford et al., 1998; Modolo et al., 2007; Owens and Mclntosh, 2009). The $K_{m}$ of UGT78K1 for kaempferol (174 $\mu \mathrm{M})$ was within this range. Interestingly, UF3GTs that are inhibited by cyanidin $(174-400 \mu M)$ (Saleh et al., 1976a; Saleh et al., $1976 \mathrm{~b}$; the present study) have greater $K_{m}$ values for flavonoid substrates than UF3GTs that do not exhibit substrate inhibition (12-90 $\mu \mathrm{M})$ (Ford et al., 1998; Modolo et al., 2007; Owens and McIntosh, 2009). The identification of more UF3GTs and characterization of their kinetics for cyanidin may help determine whether there is a relationship between $K_{m}$ and inhibition.

Anthocyanins accumulate to visible levels in the seed coat, mature stem, and purple flower petals of black soybean cv. Clark. By contrast, flavonol glycosides have been reported to accumulate in all aerial organs of soybean (Graham, 1991). UGT78K1 was expressed transcriptionally in all organs, not just the sites 
of visible anthocyanin accumulations (Figure 3.3). This expression pattern is similar to the UGT78D2 gene (At5g17050) in Arabidopsis (Tohge et al., 2005; Kubo et al., 2007) and may suggest that UGT78K1 also has a role in anthocyanin and flavonol glycoside biosyntheses in vivo.

UGT78K1 was demonstrated to catalyze the transfer of galactose to the 3position of cyanidin or delphinidin at low rates relative to the corresponding glucosyltransferase reactions (Figure 3.6). These findings are consistent with previous reports of mainly 3-O-glucosylated and minor amounts of 3-Ogalactosylated cyanidins and delphinidins in the seed coat of black soybean (Lee et al., 2009). However, knock-out or suppression of UGT78K1 would be needed to determine whether this minor activity has a role in the accumulation of anthocyanin galactosides in the seed coat of black soybean. We are currently attempting to obtain black soybean lines expressing an RNAi vector for silencing the UGT78K1 gene to test this hypothesis and to potentially change the color of the soybean seed coat.

\subsection{Concluding Remarks}

In conclusion, we have isolated a cDNA from the seed coat of black soybean and have constructed a recombinant enzyme expression plasmid and transgenic Arabidopsis lines to determine the function of the encoded enzyme. Our results suggest that UGT78K1 is a UF3GT involved in anthocyanin and flavonol glycoside biosynthesis. Engineering reduced anthocyanin levels in the seed coat of black soybean by suppressing the expression of UGT78K1 could potentially 
produce distinct seed colors to enable the visual identification and monitoring of transgenic grains (Kovinich et al., 2011a). However, RT-PCR identified the coexpression of Glyma08g07130 (Figure 3.3), which was demonstrated to be closely related to UGT78K1 by phylogenetic analyses (Figure 3.2 ) and may suggest functional redundancy in this tissue. Future work will determine the acceptor substrate specificities of recombinant Glyma08g07130 for anthocyanidins to determine whether overlapping substrate specificities exist with UGT78K1. This will determine whether both genes must be silenced to reduce anthocyanin accumulations in the seed coat of black soybean. 


\section{CHAPTER 4}

A combined analysis of transcriptome and metabolite data to identify pigment genes and to understand the molecular control of black soybean grain color

\section{Adapted from:}

Kovinich $\mathrm{N}^{1}$, Saleem $\mathrm{A}^{3}$, Arnason $\mathrm{JT}^{2}$, and Miki $\mathrm{B}^{2}$. 2011. Combined analysis of transcriptome and metabolite data reveals extensive differences between black and brown nearly-isogenic soybean (Glycine max) seed coats enabling the identification of pigment isogenes. BMC genomics 12:381. doi:10.1186/14712164-12-381.

${ }^{1}$ Designed and conducted research, analyzed data, and wrote the manuscript for publication

${ }^{2}$ Obtained funding for the research and revised the manuscript ${ }^{3}$ Developed the HPLC-DAD method

\subsection{Introduction}

Flavonoids represent a class of plant secondary metabolites that have evolved a variety of physiological functions including pigmentation, pathogen defense, and UV protection (Winkel-Shirley, 2001). Additionally, metabolic engineering of flavonoids has become an important target for plant biotechnology, as flavonoids provide health benefits to foods, favorable agronomic traits to crops, and may be used in the future to color commercial transgenic materials such as grains to facilitate their identification and monitoring (Dixon, 2005; Butelli et al., 2008; Kovinich et al., 2011a).

Commercial soybean (Glycine max (L.) Merr.) has a yellow grain. However rare spontaneous mutants exist that have black (iRT) or brown (irT) seed coat 
(testa) color phenotypes. Black (iRT) and brown (irT) soybean seed coats contain proanthocyanidins (PAs, a.k.a. condensed tannins) but differ in the presence/absence of anthocyanins (Todd and Vodkin, 1993). A goal for biosafety is to engineer a novel red seed coat color as a marker for transgenic soybean grains to facilitate their identification (Kovinich et al., 2011a), and could potentially be achieved by the suppression of anthocyanin-specific genes that are overexpressed in the black soybean seed coat. However the genes have not yet been identified.

Six genetic loci $(I, R, T, W p, W 1$, and $O)$ (Bernard and Weiss, 1973) identified by classical genetics control flavonoid-based seed coat color in soybean. The I locus controls the presence or absence and spatial distribution of flavonoid pigments and has four alleles $\left(I, l^{i}, l^{k}, I\right)$; I gives completely non-pigmented seed coat, $l^{\prime}$ restricts pigment to the hilum and $l^{k}$ to a saddle-shaped region, whereas the $i$ allele results in a fully pigmented seed coat (Bernard and Weiss, 1973). The recessive $i$ allele results from spontaneous deletion of $\mathrm{CHS} 4$ or $\mathrm{CHS} 1$ promoter sequences and results in the abolishment of a posttranscriptional RNA silencing mechanism that results in the increased accumulation of chalcone synthase (CHS) transcripts in the seed coat (Todd and Vodkin, 1996; Tuteja et al., 2004).

The $T$ locus is a pleiotropic locus that controls the type and abundance of flavonoid pigments in the seed coat in addition to other traits such as seed coat cracking and trichome pigmentation (Woodworth, 1921; Buzzell et al., 1987; Todd and Vodkin, 1993). Genetic polymorphisms that affect the expression or 
function of the flavonoid $3^{\prime}$-hydroxylase gene $\left(F 3^{\prime} H 1\right)$ have been shown to cosegregate with recessive $t$ alleles (Toda et al., 2002; Zabala and Vodkin, 2003).

The W1 locus controls flower color and affects seed color only in an iRt background; where $W 1$ and $w 1$ alleles give imperfect black and buff seed coat colors, respectively (Bernard and Weiss, 1973). The W1 allele for purple flower color was shown to code flavonoid $3^{\prime}, 5^{\prime}$-hydroxylase $\left(\mathrm{F}^{\prime} 5^{\prime} \mathrm{H}\right)$, as a $65-\mathrm{bp}$ insertion in the gene $\left(F 3^{\prime} 5^{\prime} H\right)$ co-segregated with white flower color (w1) (Zabala and Vodkin, 2007).

The Wp locus was suggested to code the flavonone 3-hydroxylase gene (F3H1) by microarray analysis as high levels of $F 3 H 1$ transcripts co-segregated with purple flower color $(W p)$, and low levels with pink (wp) flowers (Zabala and Vodkin, 2005). In the seed coat, recessive wp resulted in a change from black (iRTWp) to a lighter grayish (iRTwp) color (Zabala and Vodkin, 2005).

The $O$ locus affects the color of brown (irTO) seed coats, with the recessive $o$ allele giving a red-brown (irTo) phenotype (Bernard and Weiss, 1973). The $O$ locus has been suggested to code the proanthocyanidin (PA, a.k.a. condensed tannin) biosynthesis gene anthocyanidin reductase (ANR), as the gene was located between markers that flank the $O$ locus on the soybean physical map (Yang et al., 2010). However molecular genetics analyses have not yet demonstrated the identity of the $O$ locus gene.

Finally, the $R$ locus controls the presence $(R)$ or absence $(r)$ of anthocyanins in black (iRT) or brown (irT) seed coats, respectively (Nagai, 1921). Despite the identification of several pigment genes from soybean, only transcripts for 
anthocyanidin synthase (ANS) genes (ANS23-1, ANS27-1 and ANS100) have been shown to be overexpressed in the black (iRT) seed coat compared to the brown (irT) nearly-isogenic line by northern blot $(\mathrm{Ku}, 2009)$. The upregulation of several ANS genes suggests the $R$ locus to code a regulatory factor, and raises the possibility that other isogenes for anthocyanin biosynthesis may be upregulated.

Recently, a cDNA coding the UDP-glycose:flavonoid-3-O-glycosyltransferase (UF3GT) gene (UGT78K1) was isolated from the black (iRT) seed coat and shown to function in anthocyanin biosynthesis in vitro and by complementation of a gene mutation in Arabidopsis (Kovinich et al., 2010). However UGT78K1 expressions have not been investigated in relation to seed coat color.

The soybean genome sequence Glyma1 was predicted to code 46,430 protein-coding genes with nearly $75 \%$ of the genes present in multiple copies (Schmutz et al., 2010). This may suggest a relatively high frequency of functional redundancy and increased difficulty in identifying soybean genes by traditional approaches. However, using transcriptome analysis tools such as the Soybean GeneChip equipped with 37,500 probe sets in combination with broad-coverage metabolite analysis methodologies such as LC-MS/MS, gene functions could potentially be efficiently predicted. The combined analysis of transcriptome and metabolite data has been shown to be a powerful approach for the functional identification of unknown genes (Hirai et al., 2004; Hirai et al., 2005; Tohge et al., 2005). Metabolite differences caused by the overexpression of a transcription factor, the exposure to a nutritional stress, and by species differences have been 
correlated with differences in transcriptome profiles to successfully predict the functions of unknown genes in flavonoid, glucosinolate, and alkaloid biosyntheses (Hirai et al., 2004; Tohge et al., 2005; Pienkny et al., 2009).

In the present study we have employed targeted and non-targeted metabolite analysis methodologies and have demonstrated that black ( $i R T)$ and brown (irT) nearly-isogenic soybean seed coats do not just differ in the presence/absence of anthocyanins, and have extensive differences in procyanidin, (iso)flavonoid and phenylpropanoid compositions. The underlying differences in gene expressions were then identified by microarray analysis, and the putative functions of 20 unknown genes were assigned by comparison to metabolite data. From the set of differentially regulated genes, two putative late-stage anthocyanin genes were selected and the functions of their coded enzymes were validated in vitro. In addition, a set of gene candidates potentially coded by the $\mathrm{R}$ locus have been provided.

\subsection{Materials and Methods}

\subsubsection{Chemicals}

Cyanidin was purchased from Indofine (Somerville, NJ, USA) UDP-glucose and SAM from Sigma-Aldrich (Oakville, ON, CA), the 3-O-glucosides of delphinidin and petunidin from Polyphenols (Hanaveien, NO), and all other phytochemicals were purchased from Extrasynthese (Lyon, FR). All solvents for

LC-MS/MS and HPLC-DAD analyses were of HPLC grade purchased from Fisher Scientific (Ottawa, ON, Canada). 


\subsubsection{Plant Materials and Growth Conditions}

Black (iRT) soybean (G. max (L.) Merr.) variety Clark (PI547438) and the nearly isogenic brown (irT) soybean (PI 547475) were obtained from the U.S. Department of Agriculture Soybean Germplasm Collection (Agricultural Research Service, University of Illinois at Champaign-Urbana). Seeds were surfacesterilized in $2 \%$ triton $/ 70 \% \mathrm{EtOH}-\mathrm{H} 2 \mathrm{O}(7: 3, \mathrm{v} / \mathrm{v})$ for $5 \mathrm{~min}$ on a mixer wheel, rinsed three times with $\mathrm{EtOH}$, dried, and germinated in autoclaved vermiculite in a Conviron E15 cabinet with a photoperiod of $16 \mathrm{~h}$ light $\left(590 \mu \mathrm{E} \mathrm{m} \mathrm{m}^{2} \mathrm{~s}\right)$ at $25^{\circ} \mathrm{C}$, and $8 \mathrm{~h}$ dark at $20^{\circ} \mathrm{C}$. Plants were fertilized twice weekly with $\mathrm{N}: \mathrm{P}: \mathrm{K} 35-5-10$ (1\% w/v). After 12 days, seedlings were transplanted to autoclaved soil and 34 days later the photoperiod was changed to $12 \mathrm{~h}$ light/12 $\mathrm{h}$ dark to encourage reproductive development. For RNA isolation, seed coats were dissected from plants, immediately frozen in liquid $\mathrm{N}_{2}$, lyophilized, and stored at $-80^{\circ} \mathrm{C}$. Seed coats for comparative analyses were harvested on the same days, $4 \mathrm{~h}$ after the initiation of the light cycle to minimize for potential differences in transcript and metabolite accumulations that may be influenced by circadian rhythm.

\subsubsection{Seed Coat Metabolite Analyses}

Lyophilized seed coats $(\sim 40 \mathrm{mg})$ from each developmental stage were pulverized in $\mathrm{HCO}_{2} \mathrm{H}-\mathrm{MeOH}-\mathrm{H}_{2} \mathrm{O}(400 \mu \mathrm{L}, 15: 80: 5, v / v)$ using a FastPrep FP120 Homogenizer (Thermo Scientific, ON, Canada) and incubated at $4{ }^{\circ} \mathrm{C}$ for $2 \mathrm{~h}$. The slurry was centrifuged at $20,000 \mathrm{~g}$ for $10 \mathrm{~min}$, and $5 \mu \mathrm{l}$ of supernatant from 
each sample was measured by photospectroscopy using a NANODROP 2000 (Thermo Scientific, ON, Canada) according to the formula A530-0.25A657 to compensate for chlorophyll absorption at $530 \mathrm{~nm}$ (Mancinelli, 1990). The quantity of anthocyanins was determined by comparison to a standard curve and expressed as cyanidin 3-O-glucoside equivalents. For HPLC-DAD and LCQTRAP analyses, samples were extracted for an additional $24 \mathrm{~h}$ and $200 \mu \mathrm{l}$ of supernatant was vortexed with $\mathrm{HCO}_{2} \mathrm{H}-\mathrm{MeOH}(1 \mathrm{~mL}, 15: 85$, v/v) for $20 \mathrm{sec}$, centrifuged at $20,000 \mathrm{~g}$ for $10 \mathrm{~min}$, and the supernatant was evaporated under a stream of nitrogen gas (to $50 \mu \mathrm{L}$ ), filtered through PVDF (0.45 $\mu \mathrm{m}$; Millipore), and $20 \mu \mathrm{l}$ and $5 \mu \mathrm{l}$ aliquots were analyzed by HPLC-DAD and HPLC-MS/MS respectively, as described below.

For analysis of proanthocyanidins (PAs), lyophilized seed coats were ground to a powder, extracted with $70 \%$ acetone $\left(1 \mathrm{mg} \mathrm{mL}^{-1}\right)$ and analyzed as described previously (Downey et al., 2003; Kennedy and Jones, 2003) with (-)-epicatechin, (+)-catechin, and (-)-epicatechin gallate used as standards for the identification of free monomers, and procyanidin B2 used as a standard for the analysis of soluble and insoluble PA oligomer compositions. Quantitation of soluble PAs by reaction with DMACA reagent and insoluble PAs by acid-catalyzed cleavage was performed as described (Li et al., 1996) using procyanidin B2 and cyanidin as standards for the respective assays.

4.2.4 Microarray Analysis, Semi-qRT-PCR, and $q R T-P C R$ 
Total RNA was isolated from pigmented soybean seed coats as described previously (Wang and Vodkin, 1994). The quantity and purity of RNA samples were determined by spectrophotometry using a NANODROP 2000 (Thermo Scientific). RNA integrity was confirmed by microfluidics using a Bioanalyser 2100 equipped with an RNA 6000 Nano Chip (Agilent Technologies Inc., Montreal, QC, Canada).

Microarray was performed on RNA isolated from three plants of each genotype. Briefly, $100 \mathrm{ng}$ of total RNA was in vitro transcribed for $16 \mathrm{~h}$ using the GeneChip 3'IVT Express Kit (Affymetrix, http://www.affymetrix.com). Labelled RNA was hybridized to GeneChip Soybean Genome Arrays as described by the manufacturer (Affymetrix). Statistical analyses of data for the identification of differentially regulated genes were performed using FlexArray software (M. Blazejczyk and associates; Genome Quebec, Montreal) that uses R and BioConductor (Gentleman et al., 2004). The raw data was adjusted for background signal, and normalized across all GeneChips using the Robust Multi-array Average (RMA) method (Irizarry et al., 2003). To identify differentially expressed genes, the SAM algorithm, which minimizes variation across arrays and incorporates an estimate of false discovery rate (FDR) (Benjamini and Hochberg, 1995 ), was used. Only genes with a fold change $>2$ or $<0.5$ and a $P$ value $<0.01$ were considered to be differentially expressed. All materials and procedures comply with the MIAME standards for array data (Brazma et al., 2001). The full dataset has been deposited in the Gene Expression Omnibus [GEO:GSE26208]. 
For semi-quantitative RT-PCR (semi-qRT-PCR), RNA samples $(3 \mu \mathrm{g})$ were treated with DNase I (Amplification grade, Invitrogen) at $37^{\circ} \mathrm{C}$ for $15 \mathrm{~min}$ to remove contaminating DNA. Reactions $(20 \mu)$ were terminated by heating $\left(65^{\circ} \mathrm{C}\right.$ for $10 \mathrm{~min}$ ) in the presence of $1 \mathrm{ul}$ of $25 \mathrm{mM}$ EDTA. First-strand cDNA was synthesized using SuperScript III Reverse Transcriptase (Invitrogen) according to the manufacturer's instructions. Parallel reactions were performed in the absence of Superscript III to test for genomic DNA contamination. Gene expressions from each cDNA sample were normalized to the endogenous reference PEPC16. Semi-quantitative RT-PCR (semi-qRT-PCR) reactions $(20 \mu \mathrm{l})$ consisted of $2 \mu \mathrm{l}$ of first-strand cDNA (or untreated RNA), $400 \mathrm{nM}$ primers, $200 \mu \mathrm{M}$ dNTPs, $1.5 \mathrm{mM}$ $\mathrm{MgCl}_{2}, 1 \mathrm{X}$ Taq Buffer, 2.5 units Taq polymerase (Fermentas). PCR cycling was $94{ }^{\circ} \mathrm{C}$ for $2 \mathrm{~min}$, followed by 23,25 , and 27 cycles for $\mathrm{CHS} 7 / \mathrm{CHS} 8$ or $27,30,33$ cycles for all other genes, $94{ }^{\circ} \mathrm{C}$ for $15 \mathrm{sec}, 58^{\circ} \mathrm{C}$ for $1 \mathrm{~min}$, and $72{ }^{\circ} \mathrm{C} 1 \mathrm{~min}$.

For quantitative RT-PCR (qRT-PCR), cDNA template was prepared as described above. Reactions $(25 \mu \mathrm{l})$ consisted of $2 \mu \mathrm{l}$ of first-strand cDNA (or untreated RNA), $250 \mathrm{nM}$ of forward and reverse primers, and $12.5 \mu$ l of the iQ SYBR Green Supermix (BioRad, ON, Canada). qRT-PCR of each target gene for each seed coat sample was performed in triplicate on cDNA samples or untreated RNA samples using an PTC-200 Peltier thermal cycler equipped with a Chromo4 continuous fluorescence detector (MJ research, QC, Canada). PCR cycling was $95{ }^{\circ} \mathrm{C}$ for $10 \mathrm{~min}$, followed by 40 cycles of $95^{\circ} \mathrm{C}$ for $30 \mathrm{sec}, 58^{\circ} \mathrm{C}$ for $1 \mathrm{~min}$, and $72{ }^{\circ} \mathrm{C} 1 \mathrm{~min}$. qRT-PCR data and PCR efficiencies were analyzed using the Opticon Monitor 3 software (BioRad, ON, Canada). To verify the 
specificity of the RT-PCR reactions, melting curves were performed subsequent to each reaction in addition to fractionation of RT-PCR products on agarose gels. Semi-qRT-PCR and qRT-PCR experiments were performed in triplicate. Primers used in this study can be found in Appendix A.

\subsubsection{Cloning of UGT78K2 and OMT5 cDNAs from the Seed Coat of Black} Soybean

To clone the full-length coding sequences (CDSs) of UGT78K2 and OMT5, PCR primers were designed based on the transcript sequence predicted for Glyma08g07130 (www.phytozome.net/soybean) and based on the mRNA sequence of BT098523, respectively. Full-length CDSs for UGT78K2 and OMT5 were amplified from black (iRT) seed coat cDNA by end-to-end PCR using primers UHF/UAR and HO5F/HO5R, respectively (Appendix A) and a highfidelity DNA polymerase (Invitrogen). The resulting UGT78K2 and OMT5 amplicons (1466 bp and $836 \mathrm{bp}$, respectively) were cloned into Ndel and BamHI sites, and Ndel and Xhol sites of the pET-14b vector (Novagen, ON, Canada), respectively, and sequenced to confirm their identities.

\subsubsection{Expression of Recombinant Proteins in E. coli}

The full-length CDSs of UGT78K2 and OMT5 were independently fused inframe to the $\mathrm{N}$-terminal hexahistidine tag encoded by the $\mathrm{pET}-14 \mathrm{~b}$ vector (above). Plasmids were transformed into the expression host $E$. coli BL21(DE3) pLysS (Novagen, ON, Canada) and a single colony for each plasmid was 
selected for production of recombinant proteins. Soluble recombinant proteins were isolated following growth and induction of E. coli BL21(DE3) pLysS at 16 ${ }^{\circ} \mathrm{C}$. Recombinant proteins were purified by ion metal-affinity chromatography (IMAC) using a kit (Novagen, ON, Canada). To confirm the purity of the recombinant enzyme, the eluted fractions were visualized on $12.5 \%$ acrylamide gel stained with $0.25 \%$ Coomassie Blue. The amount of purified recombinant enzyme was determined using the BioRad reagent (BioRad, ON, Canada).

\subsubsection{Recombinant Enzyme Assays}

The UGT78K2 assay (total volume $100 \mu \mathrm{l}$ ) was performed as described previously (Kovinich et al., 2010) and consisted of recombinant enzyme (2 $\mu \mathrm{g})$, cyanidin $(20 \mu \mathrm{M})$, and UDPG $(5 \mathrm{mM})$ in assay solution. The OMT 5 assay (total volume $200 \mu \mathrm{l})$ consisted of recombinant enzyme $(3 \mu \mathrm{g})$, SAM $(100 \mu \mathrm{M}), \mathrm{MgCl}_{2}$ $(150 \mu \mathrm{M})$, Tris $\mathrm{pH} 7.5(50 \mathrm{mM})$, and 2-mercaptoethanol (14 mM). UGT78K2 and

OMT 5 assays were incubated for $5 \mathrm{~min}$ at $30^{\circ} \mathrm{C}$ and $1 \mathrm{~h}$ at $35^{\circ} \mathrm{C}$, respectively. Reactions were stopped by vortexing in $\mathrm{MeOH}(500 \mu \mathrm{l})$ for $20 \mathrm{~s}$. Reaction products were identified by comparison to commercial standards dissolved in $\mathrm{MeOH}$. Reactions were prepared for HPLC by centrifugation $(21,000 \mathrm{~g}$ for $4 \mathrm{~min}$ at $4{ }^{\circ} \mathrm{C}$ ) followed by concentration of the supernatant to a final volume of $50 \mu \mathrm{l}$ under a stream of $\mathrm{N} 2$ gas. All reactions were filtered through PTFE $(0.45 \mu \mathrm{m}$; Millipore, MA USA) and $20 \mu$ laliquots were analyzed by HPLC-DAD. Assays were performed in triplicate and experiments repeated thrice. 


\subsubsection{HPLC-DAD and HPLC-MS/MS Analyses}

HPLC-DAD analysis of anthocyanins was performed as described previously (Kovinich et al., 2010). Briefly, separations were achieved at $45^{\circ} \mathrm{C}$ on a Luna $\mathrm{C} 18(2), 4.6 \times 150 \mathrm{~mm}$, particle size $5 \mu \mathrm{m}$ fitted with a corresponding guardcolumn (Phenonenex Inc, Santa Ana, CA) using an Agilent 1100 series (Agilent Technologies Inc., Montreal, QC, Canada) equipped with an autosampler with a $100 \mu \mathrm{L}$ loop, a quaternary pump (maximum pressure, 400 bars), a column thermostat, and a diode array detector (DAD). The mobile phase consisted of $5 \% \mathrm{HCO}_{2} \mathrm{H}$ in $\mathrm{H}_{2} \mathrm{O}$ (solvent $\mathrm{A}$ ) and $\mathrm{MeOH}$ (solvent B). Optimized elution conditions were a linear gradient of $10-100 \%$ B in 25 min with a flow rate of $1 \mathrm{ml}$ $\min ^{-1}$, the column was washed for 10 min with $100 \% \mathrm{~B}$, brought back to starting mobile phase composition in $0.1 \mathrm{~min}$ and equilibrated for $5 \mathrm{~min}$ prior to the next injection. The HPLC separations were monitored at 520, 476, 350, 280, and 254 $\mathrm{nm}$. The relative quantities of anthocyanins were calculated based on percent area of each peak eluting at specific retention times.

The HPLC-ESI-MS/MS system consisted of an Agilent 1200 series (Agilent Technologies Inc., Montreal, QC, Canada) connected directly to a 3200QTRAP (ABI Sciex, Toronto, Canada). The software used for data acquisition and analysis is Analyst 1.4.2. The chromatographic conditions were the same as described for the HPLC-DAD analysis anthocyanins. The mobile phase was split $50 / 50$ post-column. For enhanced mass scan (EMS), the MS was operated in positive polarity at a scan rate of $4000 \mathrm{amu} \mathrm{s}^{-1}$ within the mass range of $100-$ $1000 \mathrm{amu}$. The optimal source conditions of the mass spectrometer were: 
collision gas high, curtain gas (N2) $25 \mathrm{~L} \mathrm{~min}^{-1}$, ion spray voltage $4500 \mathrm{~V}$, source temperature $500^{\circ} \mathrm{C}$, source gas 1 (at $50 \mathrm{psig}$ ), source gas 2 (at $55 \mathrm{psig}$ ). The optimal compound parameters were declustering potential (DP) $+20 \mathrm{~V}$, entrance potential $(E P)+10 \mathrm{~V}$, collision energy $(\mathrm{CE}) 10 \mathrm{~V}$, ionization energy $1.0 \mathrm{eV}$ and detector 2800. For enhanced product ion scan the information dependant acquisition (IDA) criteria was as follows: select $1-2$ most intense peaks which exceeds $10,000 \mathrm{cps}$, exclude targeted ions after 3 occurrences for $12 \mathrm{~s}$. Product ions were scanned within the mass range of $100-1000 \mathrm{amu}$. All source parameters were the same as for EMS. The optimal compound parameters were $\mathrm{DP}+70 \mathrm{~V}, \mathrm{EP}+10 \mathrm{~V}, \mathrm{CE} 65 \mathrm{~V}$, and collision energy spread $40 \mathrm{~V}$

\subsection{Results}

\subsection{1 $q R T-P C R$ indicates differential expressions of anthocyanin and} proanthocyanidin genes in nearly-isogenic black (iRT) and brown (irT) soybean seed coats

Among the flavonoid genes identified from soybean seed coats, only ANS transcripts were found to be overexpressed in the seed coat of black (iRT) soybean compared to the brown (irT) isoline, however no RFLP difference was observed when probing ANS genes (Ku, 2009). This suggests that ANS overexpression is not the only molecular difference between black (iRT) and brown (ir $T$ ) seed coats. To examine whether other anthocyanin genes are upregulated, transcript profiles of the recently identified UGT78K1 (Kovinich et al., 2010) and the DFR1 gene [GenBank:AF167556] were measured from seed 
coat cDNA by quantitative RT-PCR (qRT-PCR) using ANS2/ANS3 transcripts [GenBank:AY382829/GenBank: AY382830] as a positive control. Phosphenol pyruvate carboxylase (PEPC) [GenBank: D10717] was used as an endogenous reference for normalization of the qRT-PCR measurements of target genes across seed coat samples, as PEPC is expressed at similar levels in a wide range of soybean tissues and has been used previously as a reference for gene expressions during soybean seed coat development (Sugimoto et al., 1992; Tuteja et al., 2004).

qRT-PCR demonstrated UGT78K1 to be significantly overexpressed in the black (iRT) seed coat at the transition stage ( $300 \mathrm{mg}-400 \mathrm{mg} \mathrm{FSW}$ ) and early maturation stage (400 - $500 \mathrm{mg} \mathrm{FSW}$ ) of seed development compared to the brown (irT) isoline, and to be almost undetectable at earlier stages (Figure 4.1). Stereomicroscopy and photospectroscopic measurements confirmed these developmental stages to coincide with the stages of anthocyanin biosynthesis in the black (irT) seed coat (Figure 4.2). In contrast to UGT78K1, DFR1 was not differentially expressed at the stages of anthocyanin biosynthesis (Figure 4.1). ANS2IANS3 mRNAs were confirmed to be overexpressed in the black (iRT) seed coat at most stages (Figure 4.1) as shown previously (Ku, 2009).

The seed coat palisade cells of black ( $i R T$ ) soybean are unusual compared to those of other model plants used for the study of anthocyanin biosynthesis (e.g. Arabidopsis, Maize, and Petunia) because black ( $i R T$ ) soybean palisade cells synthesize both anthocyanins and proanthocyanidins (PAs). ANR and LAR 


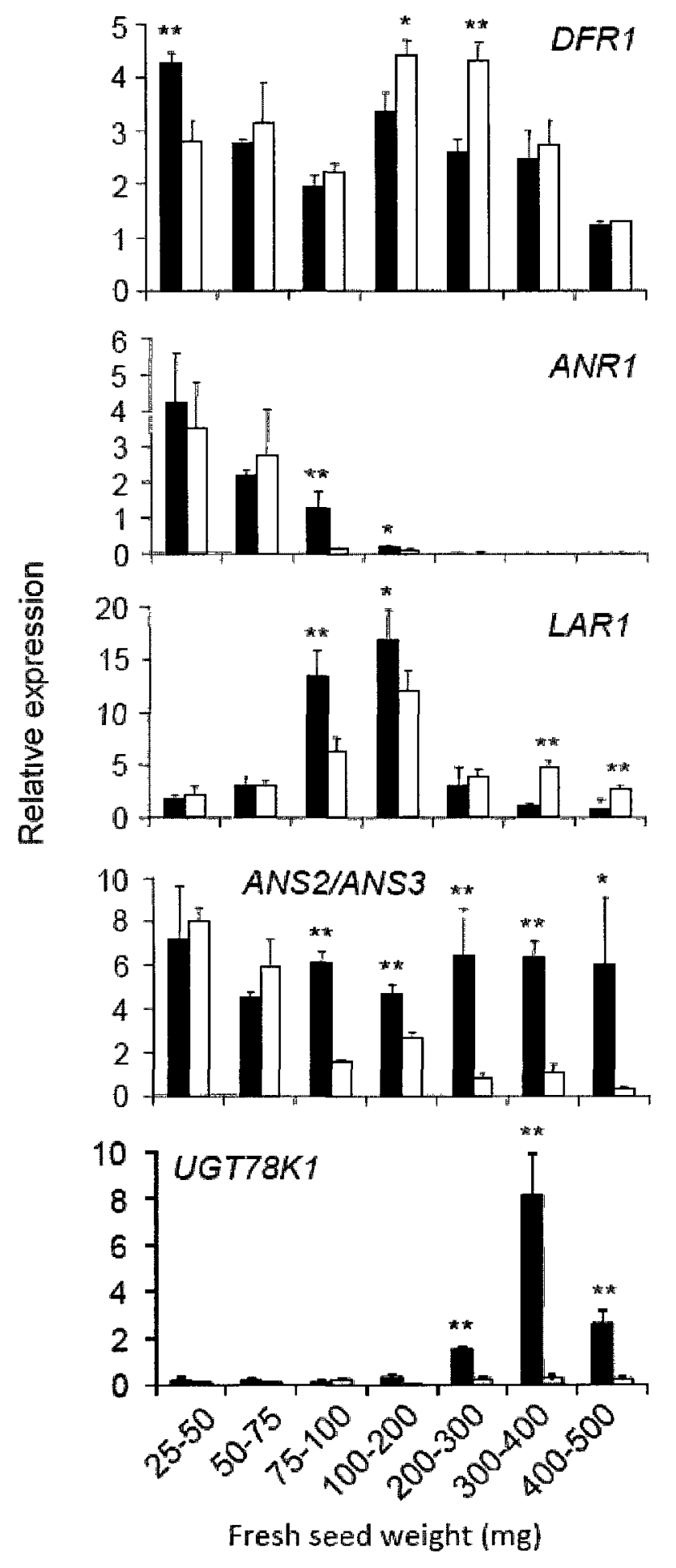

Figure 4.1. Transcript profiles from black (iRT) and brown (irT) seed coats during development measured by qRT-PCR. Black (iRT) soybean (black bars) and brown (irT) soybean (white bars). Student's t test significant at $\left.\mathrm{P}<0.01{ }^{* \star}{ }^{*}\right)$, student's $t$ test significant at $\mathrm{P}<0.05\left(^{*}\right)$. 
enzymes have been identified to catalyze the conversion of cyanidin to epicatechin and leucocyanidin to catechin, respectively, which are the putative initial biochemical steps of PA biosynthesis (Tanner et al., 2003; Xie et al., 2003). To investigate how a seed tissue may coordinate the biosynthesis of these parallel pigment pathways, the expressions of putative PA genes ANR1 [GenBank:JF433915] and LAR1 [GenBank:JF433916] were examined in relation to anthocyanin gene expressions. qRT-PCR demonstrated ANR1 expressions to decrease throughout seed coat development in both genotypes and to be almost undetectable by the stages of UGT78K1 overexpressions and anthocyanin biosynthesis (Figure 4.1). By contrast, LAR1 expressions were downregulated at the stages of anthocyanin biosynthesis in the black (iRT) seed coat (Figure 4.1). In conclusion, these results show that the recently identified UGT78K1 is upregulated in the black (iRT) seed coat, in addition to ANS2/ANS3, and that there is a simultaneous downregulation of the PA gene LAR1 at the stages of anthocyanin biosynthesis. These results demonstrate that expression differences between black ( $i R T$ ) and brown (irT) seed coats are not limited to anthocyanin genes, and may suggest extensive differences in gene expressions and phenolic compositions.

\subsubsection{Combined analysis of targeted and non-targeted methodologies indicate} overaccumulation of anthocyanins, altered procyanidin, and reduced flavonol, benzoic acid, and isoflavone compositions in the seed coat of black (iRT) soybean 
A IRT
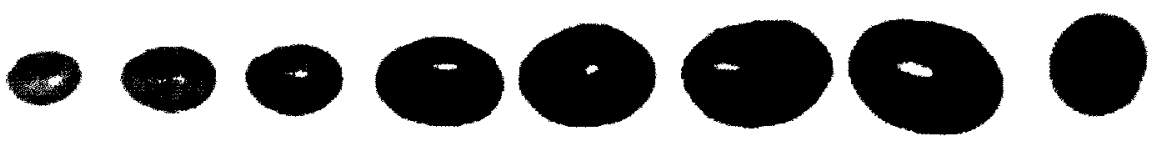

in $T$
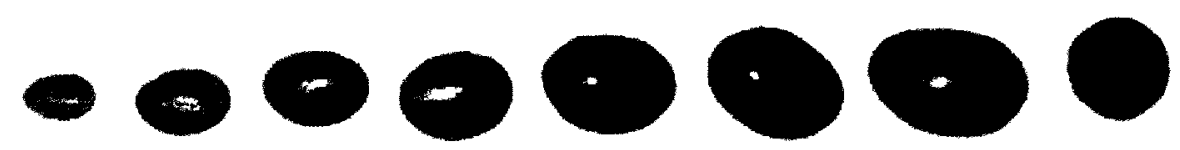

$v^{5} 5^{4}$
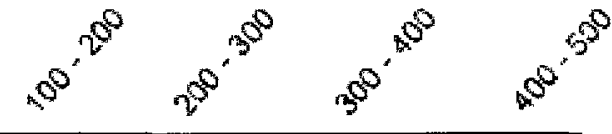

Fresh sead weight (mg)

B

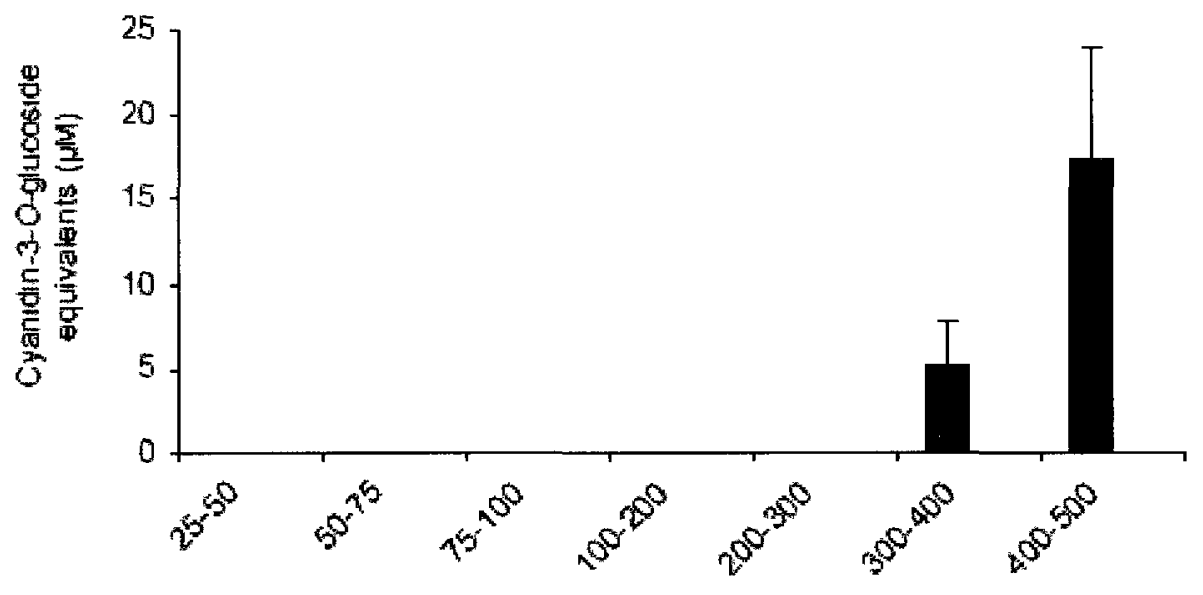

Fresh seed weight (mg)

Figure 4.2. Anthocyanin accumulations in black (iRT) and brown (irT) soybean seed coats during seed development. The fresh weight stages of seed development are indicated. (A) Seed color phenotypes during development. (B) Total amounts of anthocyanins in the seed coat of black (iRT) soybean during seed development. No anthocyanin absorbance was measured from brown (irT) seed coat extracts. 
The structures of anthocyanins from only a few (Korean) black soybean varieties have been described (Choung et al., 2001; Lee et al., 2009) and information regarding other phenolic compositions may be limited to proanthocyanidins (PAs), where acid-catalyzed hydrolysis of black (iRT) and brown (irT) seed coats showed the PAs to be 3',4'-hydroxylated (Todd and Vodkin, 1993). A more extensive analysis of phenolic composition differences between black ( $i R T)$ and brown (irT) seed coats could greatly improve our understanding of seed pigmentation, and may suggest gene activities that function in pigment biosynthesis. For these reasons, black ( $i R T$ ) and brown (irT) Clark seed coat metabolites were extracted at the transition stage $(300 \mathrm{mg}-400$ mg FSW) of seed development with multiple solvents and analyzed by targeted high performance liquid chromatography-diode array detection (HPLC-DAD) and non-targeted HPLC-tandem mass spectrometry (HPLC-MS/MS). To ensure seed coats were at the same stage of development, the days post anthesis, pod length, pod color, embryo morphology, and transcript levels of the developmental marker gene Gm-r1083-1191, a putative cutin biosynthesis gene with decreasing expression during mid- to late-development (Ranathunge et al., 2010), and the metabolism gene DFR1 were ensured to be equivalent between black ( $R T)$ and brown (irT) tissues (Appendix B).

For a targeted analysis of anthocyanins, black (iRT) and brown (irT) Clark seed coats were extracted with acidified aqueous methanol and metabolites were identified using HPLC-DAD by measuring the absorbance at $520 \mathrm{~nm}$ in comparison to authentic standards. Ten cyanic pigments were identified, the 
most abundant were the anthocyanins cyanidin-3-O-glucoside (peak 5, 43.6\% total peak area), cyanidin-3-O-galactoside (peak 4, 17.4\% peak area) and petunidin-3-O-glucoside (peak $6,14.6 \%$ total peak area) with lesser amounts of delphinidin-, pelargonidin-, and peonidin-3-O-glucosides (peaks $3,7,8 ; 8.9 \%$, $1.2 \%$, and $1.1 \%$ peak areas, respectively), the aglycone cyanidin (peak $9,3.9 \%$ peak area), the 3-deoxyanthocyanidin diosmetinidin (peak 10, 2.0\% peak area), and the two unidentified anthocyanin-like compounds (peaks 1 and 2, 1.6\% and $0.8 \%$ peak areas, respectively) (Table 4.1 ). To our knowledge this is the first report of a 3-deoxyanthocyanidin from the soybean seed coat. The structures of all identified anthocyanins are represented in Figure 4.3. By contrast, cyanic pigments were not detected from the brown (irT) seed coat at any stage of seed development (not shown).

To compare the structural features of PAs, black (iRT) and brown (irT) seed coats were extracted with $70 \%$ acetone and subjected to phloroglucinol cleavage analysis (Downey et al., 2003; Kennedy and Jones, 2003). PA free monomers, polymer subunit compositions, and mean degree of polymerization (mDP) did not differ significantly between nearly-isogenic black (iRT) and brown (irT) seed coats. The soluble PA mDP was 4 , and the subunit and monomer compositions consisted mainly of epicatechin with minor amounts of unidentified compounds (Appendix C, Panel A - Panel C). Reaction with DMACA reagent demonstrated similar amounts of soluble PAs from both black (iRT) and brown (irT) seed coats $(P=0.08)$ (Appendix $C$, Panel F). Further extraction of seed coat residues at $50^{\circ} \mathrm{C}$ in the presence of acid and phloroglucinol yielded only low levels of epicatechin- 
phloroglucinol adduct, epicatechin terminal units, and several unidentified compounds (Appendix C, Panel B), and acid catalyzed cleavage of seed coat

Table 4.1. HPLC-DAD analysis of anthocyanins from the seed coat of black (iRT) soybean variety Clark.

\begin{tabular}{llll}
\hline Peak & $\begin{array}{l}\mathrm{Rt} \\
(\mathrm{min})\end{array}$ & $\begin{array}{l}\text { Area }(\%)(520 \\
\mathrm{nm})\end{array}$ & Identity* \\
\hline 1 & 1.7 & 1.6 & unidentified compound 1 \\
2 & 2.6 & 0.8 & unidentified compound 2 \\
3 & 5.5 & 8.9 & delphinidin-3-O-glucoside \\
4 & 6.3 & 17.4 & cyanidin-3-O-galactoside \\
5 & 6.7 & 43.6 & cyanidin-3-O-glucoside \\
6 & 7.1 & 14.6 & petunidin-3-O-glucoside \\
7 & 7.4 & 1.2 & pelargonidin-3-O-glucoside \\
8 & 7.7 & 1.1 & peonidin-3-O-glucoside \\
9 & 9.5 & 3.9 & Cyanidin \\
10 & 9.7 & 2.0 & Diosmetinidin \\
\hline
\end{tabular}

${ }^{\star}$ For chemical structures see Figure 4.3. 


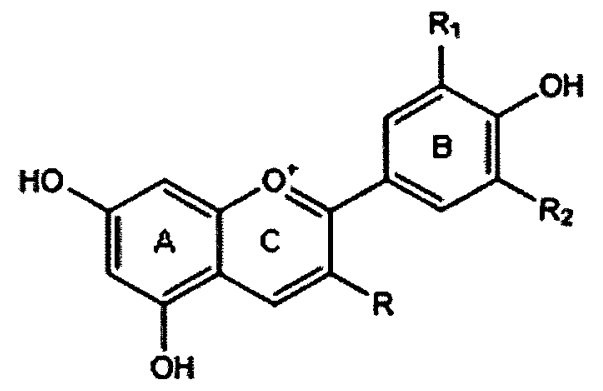

3: $R=-$ Qglucoside, $R_{1}=-O H_{1} R_{z}=-O H$ 4: $R=-O$ galactoside, $R_{1}=-O H, R_{2}=-H$

S: $R=-Q_{\text {glucoside }, R_{1}=-O H, R_{2}=-H}$

$6: R=-a_{\text {glucoside, }} R_{1}=-O \mathrm{CH}_{3} R=-\mathrm{OH}$

$7: R=-$ Qglucoside, $_{1}=-H_{1} R_{z}=-H$

8: $R=-$ aglucoside, $R_{1}=-O \mathrm{CH}_{3} R_{2}=-H$

$9: R=-O H_{1} R_{1}=-O H_{1} R_{2}=-H$

10: $R=-H, R_{1}=-\mathrm{OCH}_{3}, R_{z}=-H$

Figure 4.3. Anthocyanins from the seed coat of black (iRT) soybean variety Clark. Numbers correspond to chromatographic peaks identified by HPLC-DAD (see Table 4.1). 
residues at $95^{\circ} \mathrm{C}$ identified relatively high, but equivalent amounts of $3^{\prime}, 4^{\prime}-$ hydroxylated solvent-insoluble PAs (Appendix C, Panel E).

For a non-targeted analysis of soluble phenolic metabolites, nearly isogenic black (iRT) and brown (irT) Clark seed coats were extracted with acidified aqueous methanol and analyzed by HPLC-MS/MS over a mass range of 100 amu to $1000 \mathrm{amu}$. Identities were assigned to peaks by comparison of retention times and mass fragmentation patterns to authentic standards. Peaks with no corresponding authentic standards were identified by mass fragmentation patterns in comparison to the literature. A total of 177 peaks were detected, of which 78 peaks were assigned to 37 compounds (Table 4.2). Twenty-eight compounds were detected from the black (iRT) seed coat (Table 4.2); consisting mainly of unidentified compounds (35.7\%) and anthocyanins $(32.1 \%)$, followed by procyanidins $(25.0 \%)$. By contrast, 23 compounds were detected from the brown (irT) seed coat (Table 4.2); consisting mainly of unidentified compounds (56.5\%), followed by flavonols (13.0\%) and procyanidins (13.0\%). The majority of compounds $(62.2 \%$ of all compounds identified by HPLC-MS/MS) were exclusive to the black (iRT) or brown (irT) seed coat, suggesting extensively different biochemical specializations of these tissues. The distribution of compound classes from black ( $i R T$ ) and brown (irT) Clark seed coats is illustrated in Figure 4.4 .

The 14 compounds exclusive to the black (iRT) seed coat were anthocyanins (8 compounds), procyanidins (4 compounds), and unknown compounds ( 2 compounds) (Table 4.2). HPLC-MS/MS (Table 4.2) confirmed the identity of 
Table 4.2. HPLC-MS/MS analysis of seed coat extracts of black (iRT) and brown (irT) soybean.

\begin{tabular}{|c|c|c|c|c|c|}
\hline Compound & Identity & $\begin{array}{l}{\left[\mathrm{M}^{+}\right]} \\
(\mathrm{m} / \mathrm{z})\end{array}$ & $\begin{array}{l}\text { Major } \\
\text { fragment(s) }\end{array}$ & $\begin{array}{l}\mathrm{Rt} \\
(\mathrm{min})\end{array}$ & Genotype $^{a}$ \\
\hline 1 & Unknown 1 & 413 & 183,115 & 1.4 & $B$ \\
\hline 2 & Unknown 2 & 381 & 184,125 & 1.6 & $i r T$ \\
\hline 3 & Unknown 3 & 183 & - & 1.7 & $i R T$ \\
\hline 4 & Unknown 4 & 162 & - & 1.9 & $B$ \\
\hline 5 & Unknown 5 & 409 & 303 & 1.9 & $B$ \\
\hline 6 & Unknown 6 & 437 & 273 & 2.3 & $B$ \\
\hline 7 & Unknown 7 & 416 & - & 2.3 & $B$ \\
\hline 8 & Unknown 8 & 580 & $182,265,437$ & 2.5 & $B$ \\
\hline 9 & Unknown 9 & 507 & $485,323,132$ & 2.9 & B \\
\hline 10 & Orcinol O-hexoside & 305 & 287 & 4.0 & irT \\
\hline 11 & Unknown 10 & 331 & 248 & 4.2 & irT \\
\hline 12 & Procyanidin trimer 1 & 867 & $579,309,299$ & 4.7 & $i R T$ \\
\hline 13 & Procyanidin $\mathrm{B} 2^{*}$ & 579 & 291 & 5.5 & B \\
\hline 14 & Unknown 11 & 607 & 409,455 & 6.0 & $B$ \\
\hline 15 & Procyanidin trimer 2 & 867 & 577,407 & 6.4 & $B$ \\
\hline 16 & Delphinidin-3-O-glucoside* & 465 & 303 & 6.6 & $i R T$ \\
\hline 17 & Procyanidin trimer 3 & 867 & 579 & 6.6 & $i R T$ \\
\hline 18 & Epicatechin* & 291 & - & 6.9 & $\mathrm{~B}$ \\
\hline 19 & $\begin{array}{l}\text { Cyanidin-3-O-galactoside* } \\
\text { Daidzein O-hexoside }\end{array}$ & 449 & 287 & 7.1 & $i R T$ \\
\hline 20 & malonylated & 503 & 417 & 7.4 & irT \\
\hline 21 & Unknown 12 & 481 & 319 & 7.4 & irT \\
\hline 22 & Cyanidin-3-O-glucoside* & 449 & 287 & 7.5 & $i R T$ \\
\hline 23 & Petunidin-3-O-glucoside* & 479 & 319,303 & 8.0 & $i R T$ \\
\hline 24 & Unknown 13 & 341 & 278,111 & 8.1 & irT \\
\hline 25 & Pelargonidin-3-O-glucoside* & 433 & - & 8.3 & iRT \\
\hline 26 & Peonidin-3-O-glucoside ${ }^{*}$ & 463 & 301,286 & 8.7 & iRT \\
\hline 27 & Procyanidin dimer 2 & 579 & 409,291 & 9.2 & $\mathrm{~B}$ \\
\hline 28 & Unknown flavonol 1 & 331 & - & 9.0 & irT \\
\hline 29 & Procyanidin trimer 4 & 867 & 579,699 & 9.2 & $i R T$ \\
\hline $\begin{array}{l}30 \\
31\end{array}$ & $\begin{array}{l}\text { A-type procyanidin dimer } \\
\text { Unknown anthocyanin } 1\end{array}$ & $\begin{array}{l}593 \\
317\end{array}$ & - & $\begin{array}{l}9.7 \\
9.6\end{array}$ & $\begin{array}{l}i R T \\
i R T\end{array}$ \\
\hline 32 & Unknown flavonol 2 & 615 & - & 9.6 & $i r T$ \\
\hline 33 & Cyanidin* & 287 & - & 10.0 & $i R T$ \\
\hline 34 & Unknown 14 & 487 & - & 10.8 & B \\
\hline 35 & Diosmetinidin* & 285 & - & 10.3 & $i R T$ \\
\hline 36 & Unknown benzoic acid 1 & 633 & 331,315 & 11.0 & $\operatorname{irT}$ \\
\hline 37 & Quercetin-3-O-glucoside* & 465 & 303 & 11.0 & $\mathrm{~B}^{\mathrm{b}}$ \\
\hline
\end{tabular}

${ }^{*}$ Based on co-chromatographic and MS fragamentation comparison to an authentic standard.

a'Black soybean (iRT), brown soybean (irT), both (B).

${ }^{\mathrm{b}}$ Trace amounts in $i R T$. 


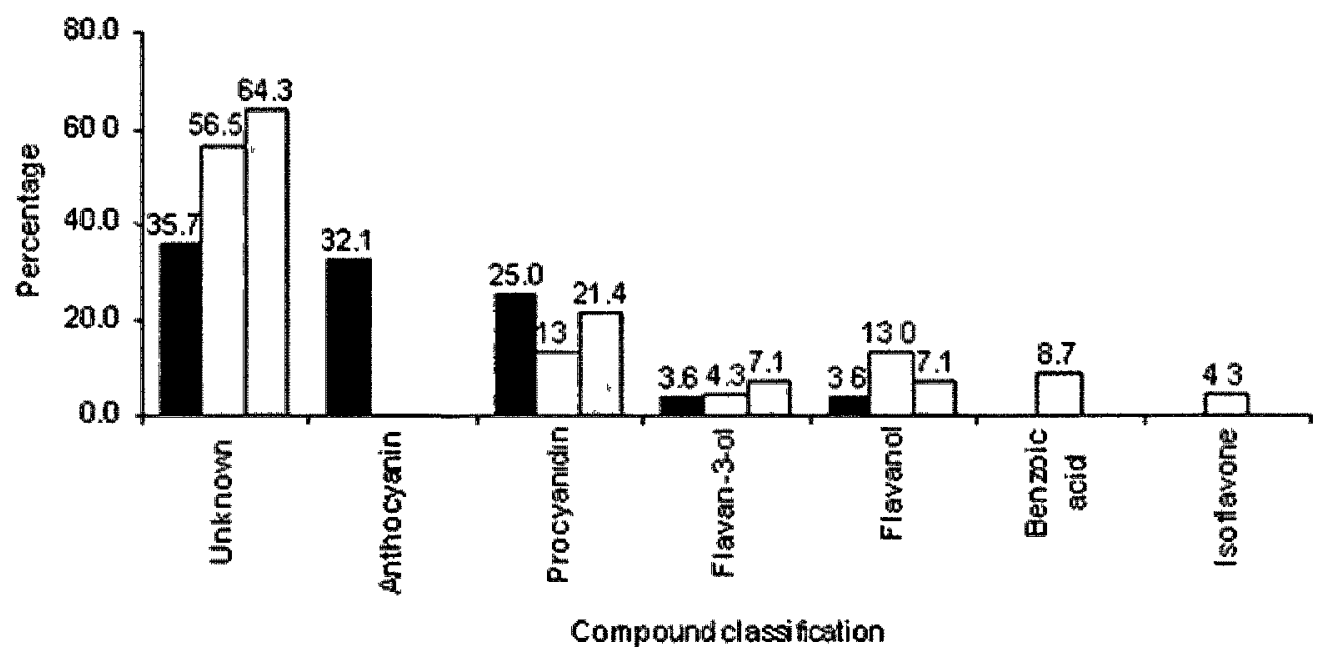

Figure 4.4. Distribution of compound classes from black (iRT) and brown (irT) soybean seed coats identified by LC-MS/MS. iRT, black bars; irT, white bars; common to both genotypes, grey bars. 
anthocyanins found by HPLC-DAD (Table 4.1). Procyanidin tetramers and larger oligomers were not detected as they exceeded the mass range (1000 amu) analyzed by the HPLC-MS/MS experiments, however 3 PA trimers (Procyanidin trimer 1,2, and 4) and the A-type procyanidin dimer were exclusive to the black (iRT) seed coat (Table 4.2).

The 9 compounds exclusive to the brown (irT) seed coat consisted of 4 unknown compounds (Unknown compound 2, 10, 12, and 13), 2 flavonols (Unknown flavonol 1 and 2), 2 benzoic acids (Orcinol O-hexoside and Unknown benzoic acid 1), and the isoflavone daidzein O-hexoside malonylated (Table 4.2).

Taken together, our metabolite analysis experiments show that nearlyisogenic black ( $i R T)$ and brown (irT) Clark seed coats have compositional differences not limited to the presence or absence of anthocyanins; with specific flavonol, isoflavone, and benzoic acids exclusive to the brown (irT) seed coat and anthocyanins and unique procyanidin oligomers exclusive to the black (iRT) seed coat. These results may suggest extensive differences in the underlying gene expressions.

\subsubsection{Microarray Analysis Indicates Concomitant Upregulation of Specific}

Phenylpropanoid, Flavonoid, and Anthocyanin Isogenes and Downregulation of Other Flavonoid, Benzoic Acid, and Isoflavone Isogenes in the Black (iRT) Seed Coat Transcriptome

Nearly $75 \%$ of genes in the soybean genome are present in multiple copies (Schmutz et al., 2010), and previously only transcripts for ANS isogenes were 
found to be overexpressed in the black ( $i R T$ ) seed coat compared to the nearlyisogenic brown (irT) tissue (Ku, 2009). For a global analysis of isogene expressions, black ( $i R T$ ) and brown (irT) Clark seed coats were dissected at the transition stage of seed development (300 - $400 \mathrm{mg} \mathrm{FSW})$ for microarray analysis using the Affymetrix Soybean GeneChip. Seed coats were ensured to be at the same stage of development as described above for the metabolite analyses (Appendix B).

Of the 37,500 probe sets present on the Soybean GeneChip, 80 were found to be upregulated more than 2-fold in black (iRT) soybean (Appendix D). Classification using the Gene-Bins ontology tool (http://bioinfoserver.rsbs.anu.edu.au /utils/GeneBins/index.php) demonstrated the majority of upregulated genes to be "unclassified without homology" $(28.7 \%)$ or "unclassified with homology" ( $24.8 \%)$, followed by "biosynthesis of secondary metabolites" (7.9\%) (Appendix E). This latter class consisted exclusively of putative (and some characterized) phenylpropanoid, flavonoid, and anthocyanin isogenes. Interestingly, putative isogenes of the phenylpropanoid pathway (4CLlike and 4CL2), the flavonoid pathway (CHS4/5, F3H-like, DFR2, ANS2/ANS3), and the late-stage anthocyanin pathway (UGT78K1, UGT78K2, OMT-like, OMT5, GST21 and GST26) were all upregulated more than 2-fold in the black (iRT) seed coat, more than 234-fold in the case of the GST26 probe set (Table 4.3).

A BLASTP search of the soybean genome sequence Glyma1 (www.phytozome.net/soybean) using the conserved plant secondary product glycosyltransferase signature sequence (PSPG box)(Hughes and Hughes, 1994) 
Table4.3. Putative phenylpropanoid/flavonoid gene sets upregulated more than 2-fold in the seed coats of black (IRT) soybean compared to the brown (IrT) isolıne ${ }^{a b}$

\begin{tabular}{|c|c|c|c|c|c|c|}
\hline \multirow[b]{2}{*}{ Gene Famıly } & \multirow[b]{2}{*}{ Probe Sets } & \multirow[b]{2}{*}{ Name } & \multirow{2}{*}{$\begin{array}{l}\text { Target } \\
\text { Description }\end{array}$} & \multirow{2}{*}{$\begin{array}{l}\text { BlackJ } \\
\text { brown fold } \\
\text { change }\end{array}$} & \multicolumn{2}{|c|}{ SAM } \\
\hline & & & & & P-value & $\begin{array}{c}\mathrm{d}- \\
\text { score }\end{array}$ \\
\hline \multirow[t]{8}{*}{$\begin{array}{l}\text { Phenylpropanold/ } \\
\text { FlavonoId pathway }\end{array}$} & GmaAffx 80720 1 S1_at & DFR2* & $\begin{array}{l}\text { Dihydroflavonol } \\
\text {-4-reductase }\end{array}$ & 6245 & 0000002 & 2470 \\
\hline & GmaAffx $421161 \mathrm{~S} 1$ at & \multirow{2}{*}{$\begin{array}{l}\mathrm{CHS4}{ }^{*} / \\
\mathrm{CHS5}^{\star}\end{array}$} & \multirow{2}{*}{$\begin{array}{l}\text { Chalcone } \\
\text { synthase } \\
\text { (LOC732575) }\end{array}$} & 1099 & 0000020 & 845 \\
\hline & GmaAffx 421161 S1_x_ & & & 806 & $262 \mathrm{E}-05$ & 712 \\
\hline & Gma 1163 1 S1_at & $\begin{array}{l}\text { ANS2*/ } \\
\text { ANS3* }^{*}\end{array}$ & $\begin{array}{l}\text { Anthocyanıdın } \\
\text { synthase }\end{array}$ & 677 & 0000018 & 877 \\
\hline & Gma 171411S1_at & F3H-Iıke & $\begin{array}{l}\text { Moderately } \\
\text { simılar to A } \\
\text { thalıana } \\
\text { putative } \\
\text { flavonone 3- } \beta \text { - } \\
\text { hydroxylase } \\
\text { clone U20744 } \\
(74 \%)\end{array}$ & 643 & 0004849 & 203 \\
\hline & Gma 7423 2 S1_a_at & 4CL-like & $\begin{array}{l}\text { 4-coumarate- } \\
\text { CoA ligase-like } \\
\text { protein }\end{array}$ & 280 & 0005815 & 196 \\
\hline & Gma 5621 1 S1_at & $\mathrm{G} 4 \mathrm{DT}^{\star}$ & $\begin{array}{l}\text { Pterocarpan 4- } \\
\text { dimethylallyltra } \\
\text { nsferase }\end{array}$ & 236 & 0009232 & 180 \\
\hline & Gma 8472 1S1_at & $4 \mathrm{CL} 2^{*}$ & $\begin{array}{l}\text { 4-Coumarate- } \\
\text { CoA ligase } 2\end{array}$ & 227 & 0000108 & 460 \\
\hline \multirow[t]{2}{*}{ Glycosyltransferase } & GmaAffx 71999 1 S1_at & $\begin{array}{l}\text { UGT78K } \\
2\end{array}$ & $\begin{array}{l}\text { Glycosyltransfe } \\
\text { rase }\end{array}$ & 309 & 0000124 & 450 \\
\hline & Gma 1002 2 S1_at & $\begin{array}{l}\text { UGT78K } \\
1^{*}\end{array}$ & $\begin{array}{l}\text { UDP- } \\
\text { glucose flavono } \\
\text { id } 3-0 \text { - } \\
\text { glucosyltransfer } \\
\text { ase }\end{array}$ & 281 & 0000229 & 403 \\
\hline \multirow[t]{2}{*}{ O-methyl-transferase } & Gma 9647 1 S1_at & OMT-like & $\begin{array}{l}\text { O- } \\
\text { methyltransfera } \\
\text { se, putatıve }\end{array}$ & 258 & 0000098 & 474 \\
\hline & GmaAffx 57777 1 S1_at & OMT5 & $\begin{array}{l}\text { Caffeoyl-CoA } \\
\text { O- } \\
\text { methyltransfera } \\
\text { se } 5\end{array}$ & 248 & 0000157 & 433 \\
\hline \multirow[t]{2}{*}{$\begin{array}{l}\text { Glutathione } \\
\text { S-transferase }\end{array}$} & GmaAffx 71212 1 A1_at & GST26 & $\begin{array}{l}\text { Glutathione S- } \\
\text { transferase }\end{array}$ & 23411 & 0000003 & 2122 \\
\hline & Gma 5139 1 S1_at & GST21 & $\begin{array}{l}\text { Glutathıone S- } \\
\text { transferase }\end{array}$ & 205 & 0000656 & 309 \\
\hline
\end{tabular}

${ }^{a}$ Expression values were obtaıned usıng RMA (Irizarry et al , 2003)

'See Appendix $D$ for the complete list of upregulated genes

*Previously characterized flavonoid biosynthesis genes 
identified 214 annotated Family 1 glycosyltransferases (UGTs)(Appendix F). Of the 214 UGTs, only 2 were found to be upregulated more than 2 -fold from the black (iRT) seed coat; the recently identified UF3GT gene UGT78K1 (Kovinich et al., 2010) was upregulated more than 2.8-fold and a second uncharacterized glycosyltransferase, which we named UGT78K2 in accordance with UGT nomenclature (Mackenzie et al., 1997), was upregulated more than 3-fold (Table 4.3).

A search of the soybean genome sequence with the conserved OMT-domain sequence (PLN02589; NCBI) and the ontology 'O-methyltransferase', identified 84 annotated OMTs (Appendix G). Of the 84 OMT genes, only probe sets for OMT-like and OMT5 were upregulated in the black (iRT) seed coat, more than 2.4-fold and 2.5-fold, respectively (Table 4.3).

A total of 52 probe sets were downregulated more than 2 -fold in the black (iRT) seed coat (i.e. black/brown $<0.5$ fold); among these, 2 probe sets by more than 10 -fold, 12 probe sets by more than 3-fold, with the remainder between 2 and 3-fold (Table 4.4; Appendix H). Classification of down-regulated genes using the Gene-Bins ontology tool demonstrated the majority to be "unclassified without homology" (45.5\%) or "unclassified with homology" (12.1\%), followed by "biosynthesis of secondary metabolites" (9.1\%) (Appendix I). The latter class consisted primarily of characterized and putative (iso)flavonoid isogenes. Isogenes for common flavonoid biosynthesis (CHS1, CHS9, F3'H), PA biosynthesis ( $L A R 1$ ), and isoflavone biosynthesis (IFR1-like) were all downregulated more than 2 -fold in the black (iRT) seed coat, more than 35 -fold in the 
Table 44 Putative phenylpropanoid/flavonoıd gene probe sets downregulated more than 2-fold in the black (IRT) compared to the brown (IIT) soybean seed coat ${ }^{\mathrm{a}}$

\begin{tabular}{|c|c|c|c|c|c|c|}
\hline \multirow[b]{2}{*}{ Gene Famıly } & \multirow[b]{2}{*}{ Probe Sets } & \multirow[b]{2}{*}{ Name } & \multirow[b]{2}{*}{ Target Description } & \multirow{2}{*}{$\begin{array}{l}\text { Black/brown } \\
\text { fold change }\end{array}$} & \multicolumn{2}{|c|}{ SAM } \\
\hline & & & & & P-value & $\begin{array}{c}d- \\
\text { score }\end{array}$ \\
\hline \multirow{8}{*}{$\begin{array}{l}\text { Phenylpropan } \\
\text {-old/FlavonoId } \\
\text { pathway }\end{array}$} & Gma 17605 1S1_at & $\mathrm{CHS}^{*}{ }^{*}$ & Chalcone synthase & 0028 & $\begin{array}{c}000000 \\
7\end{array}$ & -1645 \\
\hline & $\begin{array}{l}\text { GmaAffx } 924911 \text { S1_s_ } \\
\text { at }\end{array}$ & CHS9* & Chalcone synthase & 0047 & $\begin{array}{c}000000 \\
5\end{array}$ & -1936 \\
\hline & GmaAffx 89828 1S1_s_ & IFR1-lIke & $\begin{array}{l}\text { Highly sımılar to } \\
\text { Isoflavone reductase } \\
\text { homolog } 1 \text { (IFR1) } \\
(93 \%)\end{array}$ & 0272 & $\begin{array}{c}000013 \\
2\end{array}$ & -446 \\
\hline & Gma 8455 1S1_at & sf3'h1* & $\begin{array}{l}\text { FlavonoId } 3 '- \\
\text { hydroxylase }\end{array}$ & 0400 & $\begin{array}{c}000064 \\
6\end{array}$ & -310 \\
\hline & $\begin{array}{l}\text { GmaAffx } 932211 \text { S1_s_ } \\
\text { at }\end{array}$ & $\begin{array}{l}\text { OOMT- } \\
\text { like }\end{array}$ & $\begin{array}{l}\text { Moderately sımılar to } \\
\text { Rosa rugosa orcinol } \\
\text { O-methyltransferase } \\
\text { (OOMT-A) }(72 \%)\end{array}$ & 0410 & $\begin{array}{c}000342 \\
8\end{array}$ & -217 \\
\hline & GmaAffx 34868 1 A1_at & LAR1 & $\begin{array}{l}\text { Highly simılar to } \\
\text { Phaseolus coccineus } \\
\text { leucoanthocyanıdın } \\
\text { reductase (LAR) } \\
(88 \%)\end{array}$ & 0443 & $\begin{array}{c}000001 \\
6\end{array}$ & -902 \\
\hline & Gma 4340 2 S1_a_at & $\begin{array}{l}\text { Unknow } \\
\mathrm{n}\end{array}$ & $\begin{array}{l}\text { Moderately sımılar to } \\
P \text { trıchocarpa } \\
\text { flavonoıd O- } \\
\text { methyltransferase- } \\
\text { related }(739 \%)\end{array}$ & 0462 & $\begin{array}{c}000418 \\
0\end{array}$ & -208 \\
\hline & Gma 10949 1S1_s_at & $\begin{array}{l}\text { Unknow } \\
\mathrm{n}\end{array}$ & $\begin{array}{l}\text { Moderately similar to } \\
\text { A thalıana 3- } \\
\text { chloroallyl aldehyde } \\
\text { dehydrogenase/aldeh } \\
\text { yde dehydrogenase } \\
\text { (NAD)/conıferyl- } \\
\text { aldehyde } \\
\text { dehydrogenase } \\
\text { (ALDH2C4) }(755 \%)\end{array}$ & 0495 & $\begin{array}{c}000027 \\
3\end{array}$ & -392 \\
\hline Transport & GmaAffx 392651 S1_at & MATE1 & $\begin{array}{l}\text { Weakly sımılar to A } \\
\text { thalıana antıporter/ } \\
\text { drug transporter } \\
\text { MATE famıly proteın } \\
(698 \%)\end{array}$ & 0414 & $\begin{array}{c}000026 \\
5\end{array}$ & -395 \\
\hline
\end{tabular}

${ }^{a}$ Expression values were obtained usıng RMA (Irizarry et al , 2003)

${ }^{b}$ See Table S5 for the complete list of up-regulated genes

*Previously characterized flavonoıd bıosynthesis genes 
case of the CHS1 (Table 4.4). The flavonoid genes CHS9 and F3'H were downregulated more than 21 -fold and 2.5 -fold, respectively, the putative isoflavone gene IFR1-like by more than 3.6-fold, and the putative PA gene $L A R 1$ by more than 2.2-fold (Table 4.4).

Of the differentially regulated probe sets, 12 represented transcription factor genes, 8 were upregulated and 4 downregulated (Appendix D and Appendix I, respectively), however none of these genes clustered with known flavonoid transcription factor orthologs by phylogenetic analysis (not shown).

A previous study mapped the $\mathrm{R}$ locus between markers A668_1 and K387_1 on MLG K (Chromosome 9) (Song et al., 2004). Nineteen differentially-regulated probe sets were associated with genes located on chromosome 9 of the soybean genome sequence Glyma1 (Appendix J). Interestingly, all genes were located in a $5.16 \mathrm{Mb}$ region of the distal arm of chromosome 9 , and only the downregulated AP2/ERF (Glyma09g36840) and serine carboxypeptidase-like protein (Glyma09g36080) and the upregulated polyamine oxidase (Glyma09g36150) were located between markers (BARC-025669-04989 and Sat_293) that flank the putative position of the R locus (http://soybeanphysicalmap.org)(Figure 4.5).

To validate the microarray results, 28 differentially-regulated genes were selected and their relative expression profiles were measured by semiquantitative RT-PCR (semi-qRT-PCR) using black (iRT) and brown (ir $T$ ) seed coat cDNA as templates. Phosphenol pyruvate carboxylase (PEPC) was used as 
A
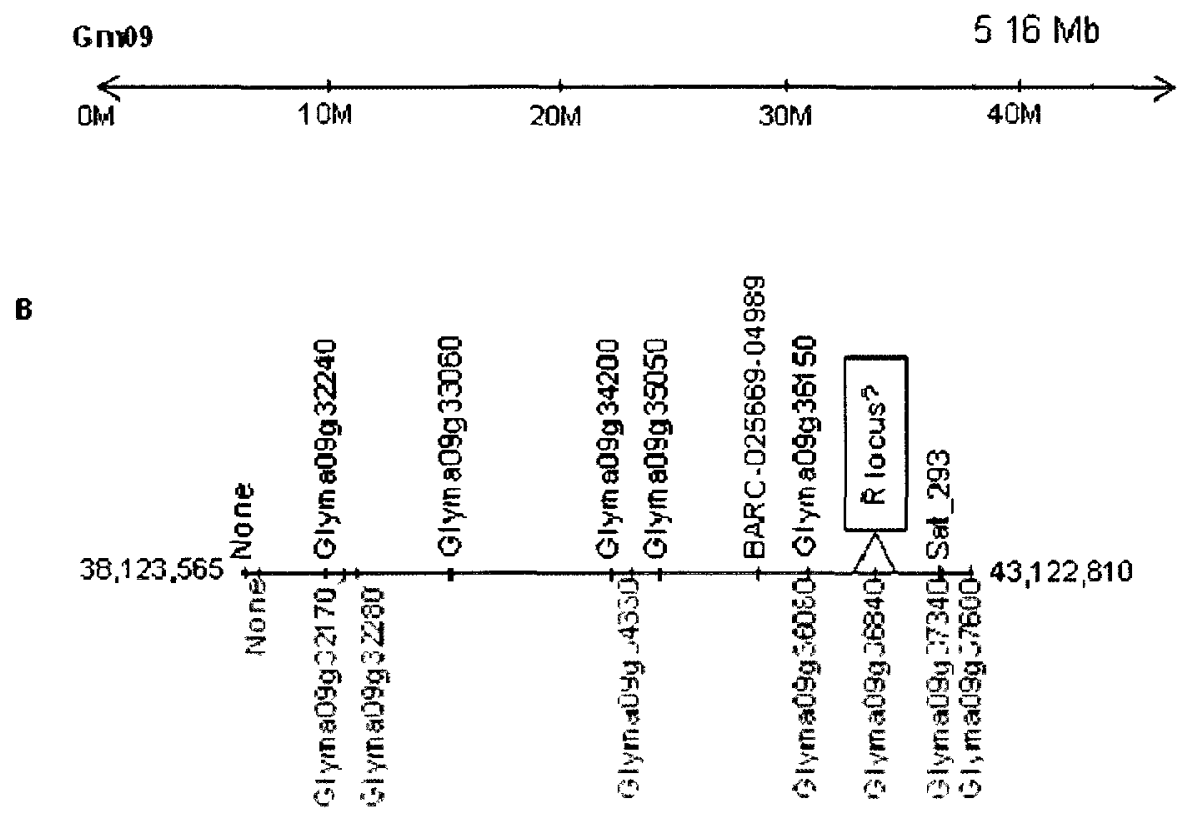

Figure 4.5. Genomic organization of differentially regulated genes from chromosome $\mathrm{Gm09}$ of the Glyma1 sequence. (A) All differentially expressed genes were located in a 5.16 Mb region of the distal end of chromosome $\mathrm{Gm} 09$. (B) Enlargement of the $5.16 \mathrm{Mb}$ region showing the locations of the upregulated genes (blue) and downegulated genes (red) relative to sequence-based markers BARC-025669-04989 and Sat_293 (black) that are situated upstream and downstream, respectively, nearest the $R$ locus in the Glyma1 sequence.

Chromosome positions of differentially regulated genes are listed in Table SX. Chromosome positions of BARC-025669-04989 (Gm09:41,620,379..41,620,751) and Sat_293 (Gm09:42,848,788..42,849,068). 
the endogenous control to ensure equal loading and the flavonoid genes CHS7/CHS8 and the putative PA gene ANR1 were used as negative controls as they were not found to be differentially expressed in the microarray analysis.

Semi-qRT-PCR confirmed the differential-expressions of all 28 genes and demonstrated similar expressions for $\mathrm{CHS7/CHS8}$ and ANR1 (Appendix K). Furthermore, as the upregulated probe set GmaAffx.42116.1.S1 did not distinguish between $\mathrm{CHS} 4$ and $\mathrm{CHS} 5$ expressions, semi-qRT-PCR was performed with gene specific primers to show the CHS4 gene, and not the CHS5 gene to be upregulated in the black (iRT) seed coat (Appendix K).

In summary, these results show that numerous specific anthocyanin, flavonoid and phenylpropanoid isogenes are upregulated and other flavonoid, benzoic acid, and isoflavone isogenes are downregulated in the black (iRT) seed coat transcriptome relative to that of the nearly-isogenic brown (irT) tissue.

\subsubsection{Putative Assignment of Function to Genes Differentially-Regulated} between Black (iRT) and Brown (irT) Seed Coats

Gene functions can be successfully predicted based on a combined analysis of transcriptome and metabolite data (Tohge et al., 2005). We compared differences in gene expressions determined by microarray to differences in metabolite compositions to assign putative functions to genes from the black (iRT) and brown (irT) seed coats (Figure 4.6). In addition to 7 previously characterized soybean flavonoid genes, several uncharacterized genes 


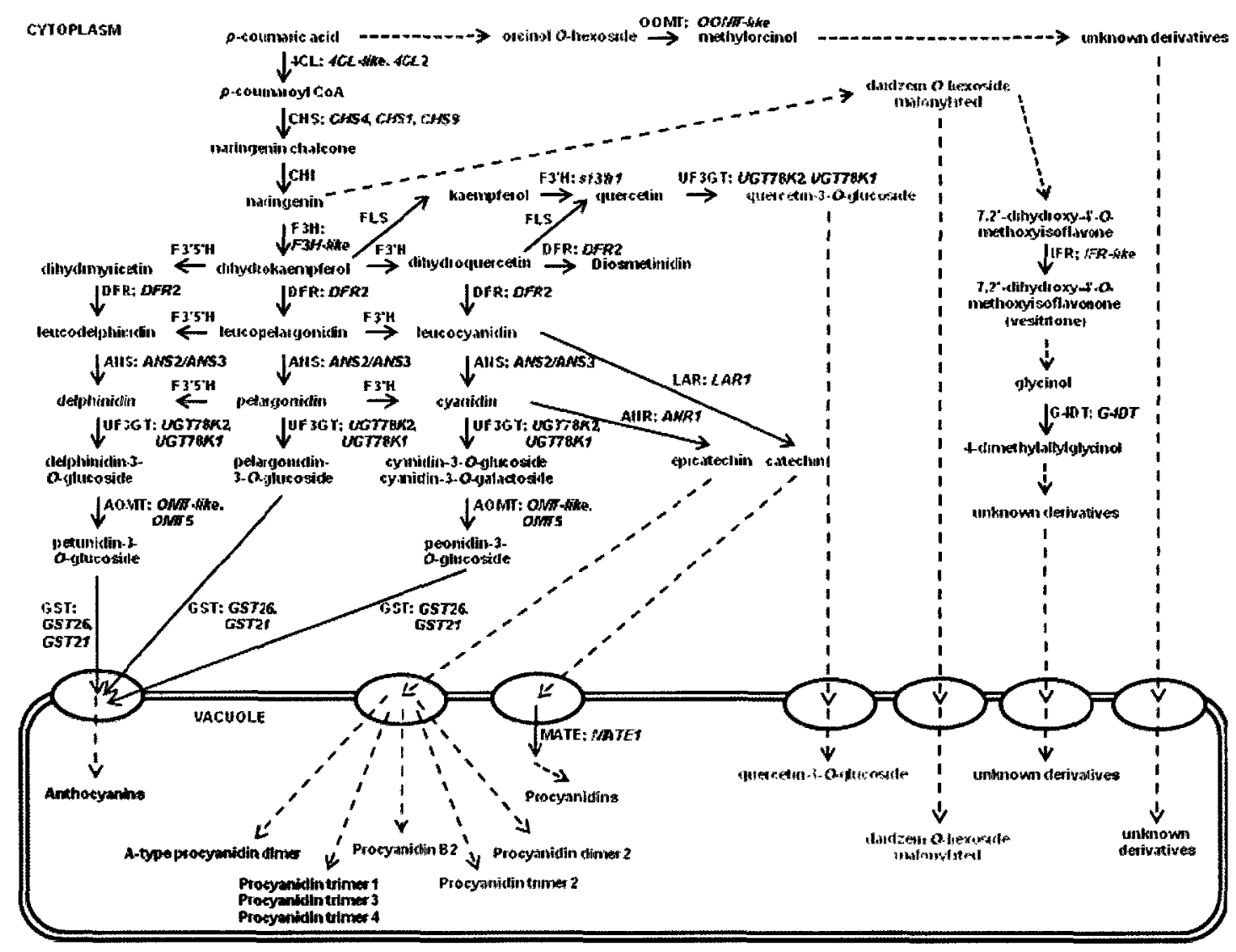

Figure 4.6. Summary of flavonoid/phenylpropanoid gene and metabolite accumulations from black and brown soybean seed coats*. Transcripts upregulated and metabolites exclusive to the black (iRT) seed coat are shown in blue. Transcripts downregulated and metabolites absent in the black (iRT) seed coat but present in the nearly-isogenic brown (irT) seed coat are shown in red. $4 \mathrm{CL}$, 4-coumarate-CoA ligase; OOMT, orcinol O-methyltransferase; CHS, chalcone synthase; $\mathrm{CHI}$, chalcone isomerase; $\mathrm{F} 3 \mathrm{H}$, flavanone 3-hydroxylase; F3'H, flavonoid 3'-hydroxylase; F3'5' $\mathrm{H}$, flavonoid 3',5'-hydroxylase; DFR, dihydroflavonol reductase; ANS, anthocyanidin synthase; UF3GT, UDPglycose:flavonoid 3-O-glycosyltransferase; AOMT, anthocyanin Omethyltransferase; GST, glutathione S-transferase; FLS, flavonol synthase; LAR, leucoanthocyanidin reductase; ANR, anthocyanidin reductase; MATE, multidrug and toxic extrusion protein; IFR, isoflavone reductase; G4DT; pterocarpan 4dimethylallyltransferase. *For the complete lists of differentially regulated transcripts see Appendix $D$ and Appendix $\mathrm{H}$, and for a complete list of differentially accumulated metabolites see Table 4.2. 
belonging to large multi-gene families with possible roles in anthocyanin biosynthesis and transport were upregulated; including one glycosyltransferase (UGT78K2), two O-methyltransferases (OMT5 and OMT-like) and two glutathione S-transferases (GST21 and GST26) (Table 3). Considering the accumulation of metabolites with specific molecular structures in the black (iRT) seed coat, putative functions of the differentially-expressed genes were assigned to specific structural modifications and transport functions (Figure 4.6).

Anthocyanins in the seed coat of black ( $i R T$ ) soybean are glycosylated at the 3-position of the C-ring (Figure 4.4). The glycosyltransferase UGT78K2 is assigned to code a UDP-glycose:flavonoid 3-O-glycosyltransferase (UF3GT) protein (Figure 4.6) and may have functional redundancies in anthocyanin biosynthesis with the previously characterized UGT78K1 (Kovinich et al., 2010), which was also upregulated in the black (iRT) seed coat (Table 4.3). Two anthocyanins (petunidin-3-O-glucoside and peonidin-3-O-glucoside) and one deoxyanthocyanidin (diosmetinidin) from the black (iRT) seed coat are methylated at the 3-position of the B-ring (the 3'-position) (Figure 4.3). The two upregulated O-methyltransferases were assigned to code anthocyanin 3'-Omethyltransferase (AOMT) proteins (Figure 4.6) and may have redundant function in anthocyanin biosynthesis this tissue. 
4.3.4 UGT78K2 and OMT5 Code UDP-Glycose:Flavonoid 3-O-

Glycosyltransferase and Anthocyanin 3'-O-Methyltransferase Proteins, Respectively

Reducing the expressions of late-stage anthocyanin genes by RNA interference (RNAi) in the seed coat of black soybean may be an effective strategy to engineer seed coat color while minimizing unintended effects on other flavonoid subpathways. However, functional redundancies may require that multiple genes be silenced in order to reduce enzyme activity enough to reduce pigment levels. Two glycosyltransferases (UGT78K1 and UGT78K2) were upregulated in the black ( $i R T$ ) seed coat relative to the nearly-isogenic brown (irT) tissue (Table 4.3) and the upregulation paralleled the accumulation of anthocyanins with 3-O-glycosylated structures (Figure 4.2), suggesting the function of these genes as UDP-glycose:flavonoid 3-O-glycosyltransferases (UF3GTs). We have characterized UGT78K1 previously and shown it to possess UF3GT activity towards anthocyanidins in vitro and in vivo (Kovinich et al., 2010). To determine the catalytic function of UGT78K2 [GenBank:HM591298], the ORF was cloned into an $\mathrm{N}$-terminal hexahistidine fusion tag vector and expressed in $E$. Coli for analysis of the coded recombinant protein in vitro. The recombinant protein (rUGT78K2) was purified by ion-metal-affinity chromatography (IMAC) had an apparent mass of $50.9 \mathrm{kDa}$ that matched well with the calculated mass of the native UGT78K2 protein $(49.04 \mathrm{kDa})$ in addition to the $2.04 \mathrm{kDa}$-terminal hexahistidine fusion tag encoded by the pET-14b vector (Figure 4.7A). In assays containing UDP-glucose as the sugar donor and cyanidin as the acceptor, the 
A

C
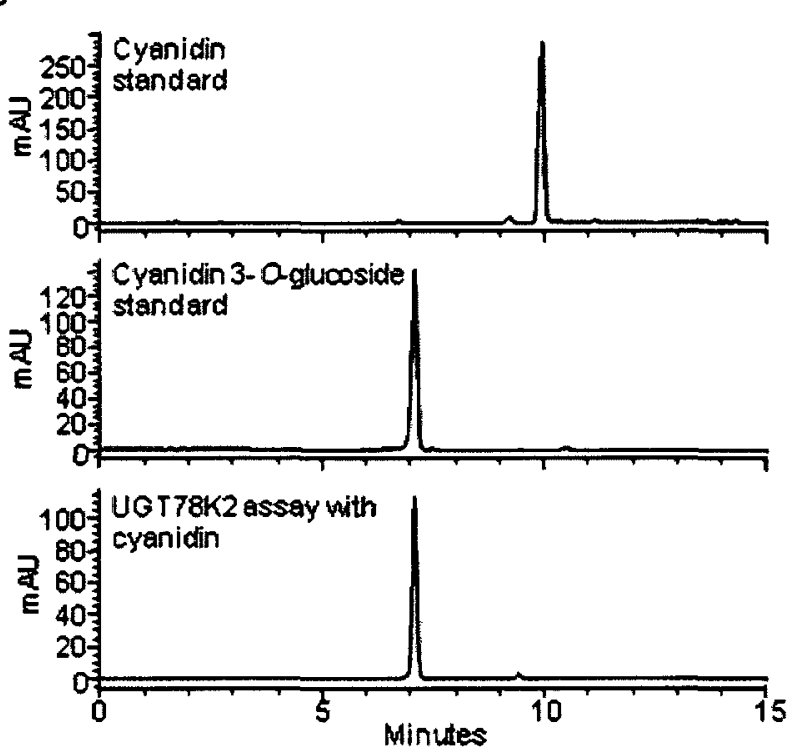

D

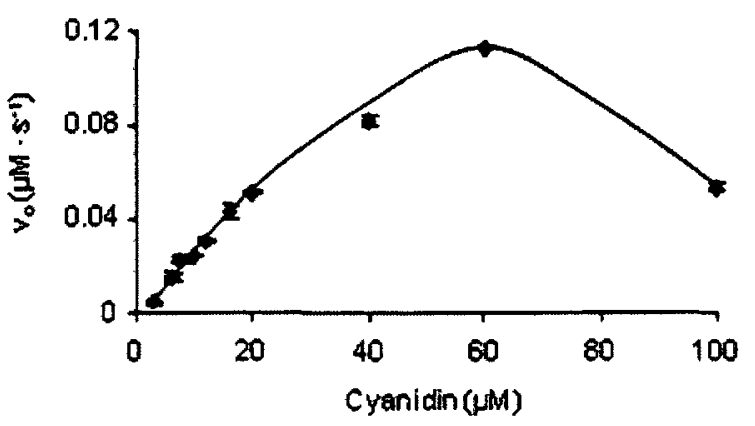

Figure 4.7. Purification of his-tagged UGT78K2 and determination of the reaction product and kinetics for cyanidin. (A) SDS-PAGE analysis of UGT78K2: total soluble protein from E. coli expressing UGT78K2 prior to induction with $100 \mathrm{mM}$ 
IPTG (lane 1), 24 h post-induction (lane 2); IMAC-purified UGT78K2 (lane 3). (B) The UGT78K2 enzyme reaction as revealed by HPLC-DAD chromatograms at $520 \mathrm{~nm}(\mathrm{C})$. HPLC retention times: cyanidin (Rt: $10.1 \mathrm{~min}$ ); cyanidin 3-Oglucoside (Rt: $7.1 \mathrm{~min}$ ). (D) UGT78K2 kinetics for the cyanidin acceptor substrate using $5 \mathrm{mM}$ UDPG as a sugar donor. Points represent the mean of three assays \pm the standard deviation.

recombinant enzyme transferred glucose to the 3-position of cyanidin to form cyanidin-3-O-glucoside (Figure 4.7B, 4.7C), whereas the boiled enzyme and bacteria expressing the corresponding empty vector could not catalyze this reaction (not shown). Similarly, in assays containing UDP-galactose as the sugar donor and cyanidin as the acceptor, the recombinant enzyme transferred galactose to the 3-position of cyanidin to form cyanidin -3-O-galactoside (not shown). Cyanidin-3-O-glucoside and cyanidin -3-O-galactoside from recombinant enzyme assays were identified by HPLC-DAD in comparison to the authentic standards, and confirmed by HPLC-MS/MS with the characteristic parent ions 449.0 [cyanidin -3-O-glucoside or cyanidin -3-O-galactoside] ${ }^{+1}$ and the MS/MS fragment $[287.3 \text { cyanidin }]^{+1}$ corresponding to the loss of glucose (-162 amu).

As we have performed the kinetics analysis for recombinant UGT78K1, we were able to employ the same experimental conditions to test the kinetics of UGT78K2. Interestingly, UGT78K2 converted cyanidin-3-O-glucoside with greater velocity than UGT78K1 and higher concentrations of cyanidin were required to inhibit its activity (Figure 4.7D) despite the enzymes having $93 \%$ amino acid similarity (Appendix $L$ ). 
Methylation of the B-ring of anthocyanins has a reddening effect on flower color (reviewed by Tanaka et al., 2009) and thus engineering the expression of 3'-Omethyltransferases could change the redness of seed color. However, despite the identification of several flavonoid OMTs from soybean, none have been shown to accept anthocyanins as substrates. Our microarray analysis identified two O-methyltransferases (OMT5 and OMT-like) to be upregulated in the black (iRT) seed coat (Table 4.3) and the upregulation paralleled the accumulation of 3'-O-methylated anthocyanins in this tissue (Figure 4.2), suggesting the function of these genes as anthocyanin 3'-O-methyltransferases (AOMTs). Following the approach described above, the ORF of OMT5 [GenBank:HQ856048] was cloned for expression and functional characterization of the corresponding recombinant protein in vitro. The purified recombinant protein had an apparent mass of 30.0 $\mathrm{kDa}$ that matched well with the calculated mass $(27.78 \mathrm{kDa})$ in addition to the $2.04 \mathrm{kDa} N$-terminal hexahistidine fusion tag encoded by the $\mathrm{pET}-14 \mathrm{~b}$ vector (Figure 4.8A). In assays containing S-adenosyl-L-methionine (SAM) as the methyl donor and cyanidin-3-O-glucoside as the acceptor, the recombinant enzyme transferred a methyl group to the 3'-position of the acceptor to form peonidin-3-O-glucoside (Figure 4.8B, 4.8C), whereas the boiled enzyme and bacteria expressing the corresponding empty vector could not catalyze this reaction (not shown). Peonidin-3-O-glucoside from recombinant enzyme assays was identified by HPLC-DAD in comparison to the authentic standard, and confirmed by HPLC-MS/MS with the characteristic parent ion 463.1 [peonidin-3O-glucoside] ${ }^{+1}$ and the MS/MS fragment 301.0 [peonidin $^{+1}$ corresponding to the 
A

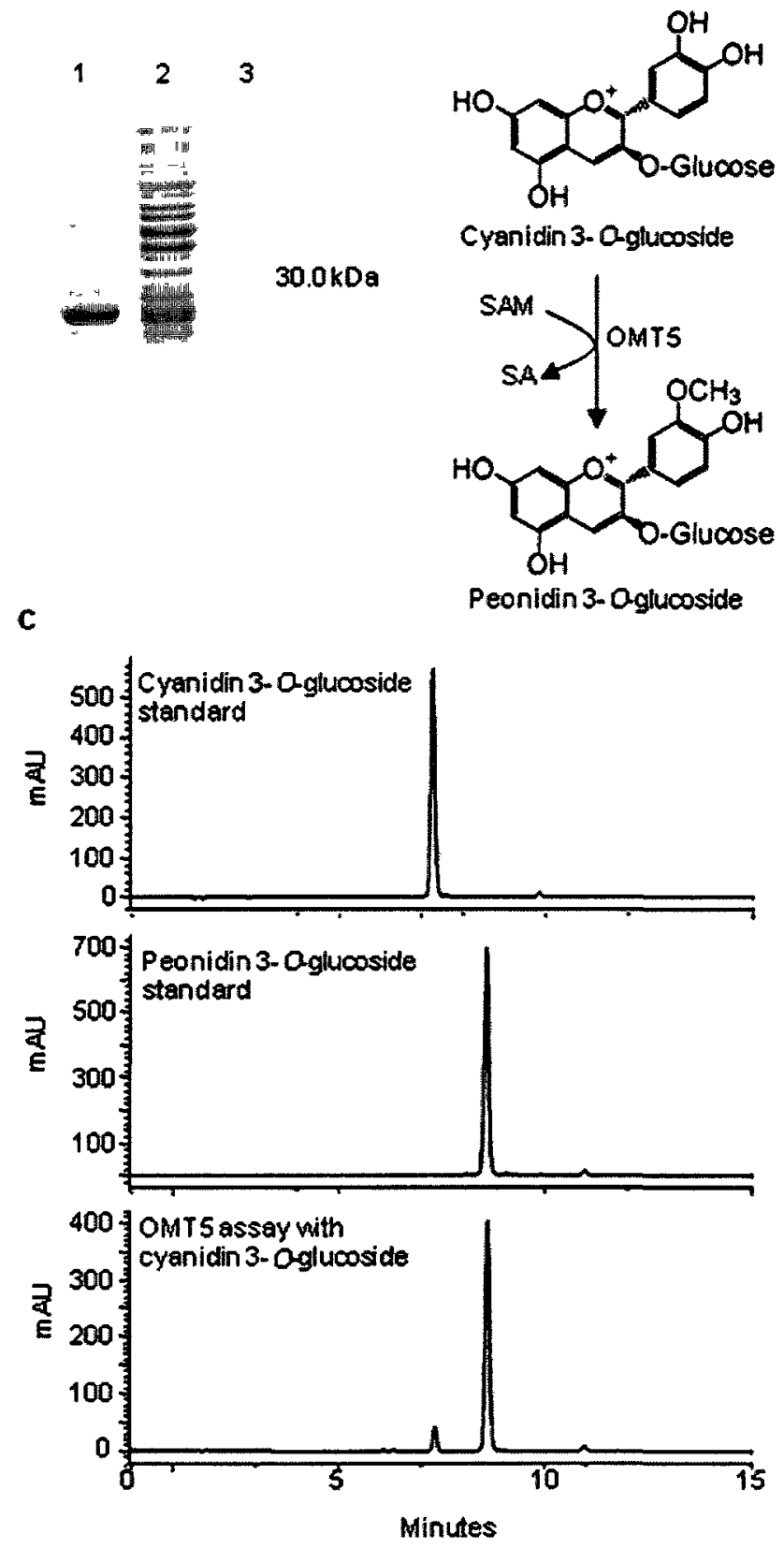

Figure 4.8. Purification of his-tagged OMT5 and determination of the reaction product for cyanidin 3-O-glucoside. (A) SDS-PAGE analysis of OMT5: total soluble protein from E. coli expressing OMT5 prior to induction with 100 IM IPTG (lane 1), 24 h post-induction (lane 2); IMAC-purified OMT5 (lane 3). (B) The OMT5 enzyme reaction as revealed by HPLC-DAD chromatograms at $520 \mathrm{~nm}$ (C). HPLC retention times: cyanidin 3-O-glucoside (Rt: $7.3 \mathrm{~min}$ ); peonidin 3-Oglucoside (Rt: $8.6 \mathrm{~min}$ ). 
loss of glucose (-162 amu).

To further investigate the function of these genes, UGT78K2 and OMT5 expression profiles were measured from seed coat cDNA throughout black (iRT) and brown (irT) soybean seed development by quantitative RT-PCR (qRT-PCR) (Figure 4.9) and expressions were compared to anthocyanin accumulation profiles throughout seed development (Figure 4.2). Similar to UGT78K1, UGT78K2 and OMT5 expressions peaked at the transition and early maturation stages of seed development ( $300 \mathrm{mg}-400 \mathrm{mg} \mathrm{FSW}, 400 \mathrm{mg}-500 \mathrm{mg} \mathrm{FSW}$ ) in the black (iRT) seed coat (Figure 4.9), the stages at which anthocyanins accumulate (Figure 4.2), and were low or almost undetectable at early stages of seed development in the black (iRT) seed coat and at all stages in the brown (irT) seed coat.

Together the qRT-PCR and recombinant enzyme assays suggest that UGT78K2 and OMT5 code UF3GT and AOMT proteins, respectively, that function in anthocyanin pigment biosynthesis in the seed of black (iRT) soybean. 


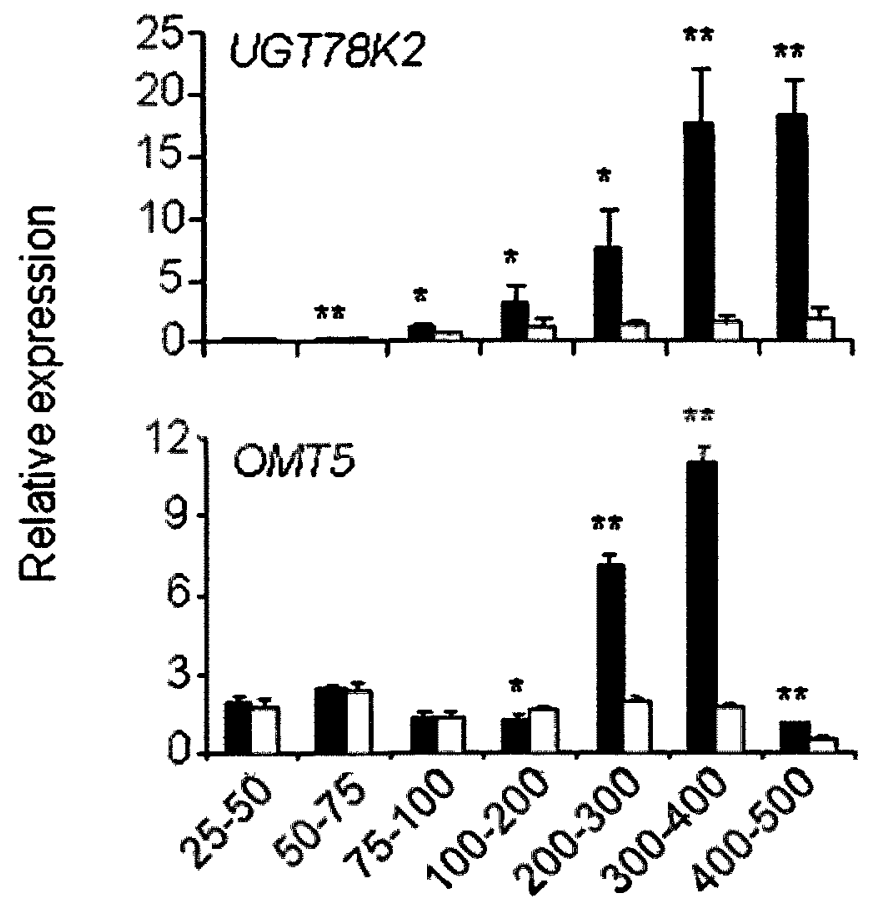

Fresh seed weight (mg)

Figure 4.9. Seed coat transcript profiles of UGT78K2 and OMT5 during seed development measured by qRT-PCR. Black (iRT) seed coat (black bars), brown (irT) seed coat (white bars). Student's $t$ test significant at $P<0.01\left({ }^{* *}\right)$, student's $t$ test significant at $\mathrm{P}<0.05\left(^{*}\right)$. 


\subsection{Discussion}

The seed coat compositions of black (iRT) and brown (irT) soybean (Glycine max) were previously known to differ only by the presence/absence of anthocyanins. However, our metabolite analysis demonstrated these nearlyisogenic tissues to have extensive differences in anthocyanin, proanthocyanidin (PA), (iso)flavonoid, and phenylpropanoid content (Table 4.2). Of the 37 compounds detected, 24 compounds ( $62.2 \%$ of all compounds identified by HPLC-MS/MS) were exclusive to either the black (iRT) or the brown (irT) seed coat. Analysis of the distributions of compound classes between seed coats demonstrated the black ( $i R T$ ) to a have a greater proportion of anthocyanins and PAs, and the brown (irT) to have a greater proportion of flavonol, benzoic acid, isoflavone, and unknown metabolite compositions (Figure 4.4). Metabolites exclusive to the black (iRT) seed coat included PA trimers (Procyanidin trimer 1, 2, and 4) and an A-type procyanidin dimer in addition to various anthocyanins (Table 4.2).

The anthocyanins identified from variety Clark were mainly similar to those reported previously from various Korean varieties (Choung et al., 2001; Lee et al., 2009) however Clark was the first shown to contain a 3-deoxyanthocyanidin (diosmetinidin) in the seed coat (Table 4.2). 3-deoxyanthocyanidins are relatively rare intensely coloured pigments found in Sorghum bicolour in response to fungal attack (Snyder and Nicholson, 1990), and from the cotyledons of yellow soybean in response to UV-C treatment (Boveris et al., 2001). The biosynthesis of 3deoxyanthocyanidins from flavonones has been shown to be a minor activity of a 
DFR isogene in Sorghum (Liu et al., 2010). It would be interesting to determine whether the DFR gene that was highly overexpressed in the black Clark seed coat (DFR2) had similar catalytic activity, and whether the Korean varieties contain genetic alterations related to $D F R 2$.

Analysis of the black (iRT) seed coat transcriptome compared to that of the nearly-isogenic brown (irT) tissue identified 80 probe sets to be upregulated (Table 4.3). This is almost double the number of probe sets upregulated by overexpression of the MYB transcription factor PAP1 in Arabidopsis (38 probe sets) (Tohge et al., 2005), similar to the number upregulated by overexpression of the anthocyanin factor LAP1 in M. Truncatula (61 probe sets), and less than half the number upregulated by overexpression of LAP1 in M. Sativa (270 probe sets) (Peel et al., 2009). The higher numbers of genes upregulated in anthocyanin-overaccumulating tissues of black soybean, $M$. truncatula and $M$. sativa likely reflect the fact that legumes have undergone genome duplication and that genes with multiple copies are frequently retained (Schmutz et al., 2010). Our data supported this hypothesis as duplicate copies of several genes were upregulated in the black (iRT) seed coat (e.g. 4CL2 and 4CL-like, UGT78K2 and UGT78K1, OMT5 and OMT-like, and GST21 and GST26).

Functional redundancies can complicate gene identification by traditional approaches. Previous to the present study, genes for only 5 flavonoid enzymes (CHS, F3H, F3'H, F3'5'H, and UF3GT) were identified to be involved in pigment biosynthesis in the soybean seed coat (Todd and Vodkin, 1996; Toda et al., 2002; Zabala and Vodkin, 2003; Zabala and Vodkin, 2005; Zabala and Vodkin, 
2007; Kovinich et al., 2010). In the present study, 20 isogenes for 14 proteins were identified to have putative roles in flavonoid biosynthesis in black (iRT) or brown (irT) seed coats using a combined analysis of microarray and metabolite data (Figure 4.6).

Transcriptome analysis has previously demonstrated a critical role of $\mathrm{CHS7}$ and CHS8 genes for isoflavonoid biosynthesis in developing embryos of soybean (Dhaubhadel et al., 2007). However, our microarray and semi-quantitative RTPCR data demonstrated that $\mathrm{CHS7}$ and $\mathrm{CHS} 8$ were not differentially regulated in black (iRT) and brown (irT) seed coats (Table 4.3). Interestingly, it was $\mathrm{CHS} 4$ that was upregulated and $\mathrm{CHS} 1$ and $\mathrm{CHS} 9$ that were downregulated in the black (iRT) seed coat (Table 4.3 and Table 4.4, respectively). As CHS4 is upregulated with anthocyanin genes, it likely has a role in anthocyanin biosynthesis in this tissue. Consistent with this, spontaneous mutations of the dominant ${ }^{i}$ allele to the recessive $i$ allele have been shown to affect the promoter region of the $\mathrm{CHS} 4$ gene (Todd and Vodkin, 1996). By contrast, the downregulation of CHS1 and CHS9 may suggest that they have a role in the parallel downregulated biochemical pathways, such as flavonol and isoflavonoid biosynthesis, as these metabolites, and transcript levels of genes for their biosynthesis, were reduced or not present in the black (iRT) seed coat (Figure 4.6). These results emphasize the complexity of isogene expressions that exist to achieve a single enzyme function in soybean.

Microarray analysis identified differentially regulated probe sets for only 3 gene functions (LAR, GST, and MATE) that have been previously shown to be 
involved in PA biosynthesis and transport. Recombinant LAR enzymes from several legume species have been shown to convert leucocyanidin to $(+)$ catechin in vitro (Tanner et al., 2003; Xie et al., 2003; Pang et al., 2007; Paolocci et al., 2007). However, the in vivo role of LAR in PA biosynthesis remains questionable as endogenous expression has been shown not to correlate with PA levels in $M$. Truncatula and heterologous expression has failed to increase PA accumulations (Pang et al., 2007). Similarly, our metabolite data showed that soybean PAs lack catechin subunits (Appendix C) and that LAR1 expressions (Figure 4.1) do not parallel PA accumulations during seed development (not shown). Interestingly, 4 PAs were found to be exclusive to the black (iRT) seed coat (Table 4.2) however the total subunit compositions, the mean degree of polymerization (mDP), and the total amounts of soluble and solvent-insoluble PAs were not different compared to the brown (irT) seed coat (Appendix C). These results suggest the PA oligomers of black (iRT) and brown (irT) seed coats may differ only in their subunit linkages. However, the potential influence of LAR or other PA genes on PA subunit linkages (if any) remains to be determined.

From the set of differentially regulated genes identified by microarray, two putative late-stage anthocyanin genes were selected and the functions of their coded enzymes were determined in vitro. UGT78K2 was found to code a UF3GT enzyme for anthocyanin biosynthesis (Figure 4.7). A UF3GT cDNA (UGT78K1) has recently been isolated from the black seed coat and functionally characterized (Kovinich et al 2010). UGT78K2 was 93\% similar to UGT78K1 but possessed increased catalytic activity towards cyanidin (Figure 4.7D). The 
identification of gene redundancy has important implications for seed coat color engineering by RNA interference, as both copies of the gene may have to be silenced to achieve a significant reduction in UF3GT activity. Similarly, two OMT genes (OMT5 and OMT-like) were found to be overexpressed in the black seed coat (Table 4.3). OMT5 was cloned and found to code an AOMT enzyme in vitro (Figure 4.8). UF3GT and OMT genes may be ideal candidates for seed coat color engineering as suppression of UF3GT genes (UGT78K1 and UGT78K2) may result in a reduction in anthocyanin levels similar to that caused by a T-DNA insertion in the Arabidopsis UF3GT (Tohge et al., 2005) and upregulation of an AOMT may result in a more red seed coat, as methylation of the B-ring of anthocyanins has a reddening effect on flower color (reviewed by Tanaka et al., 2009). However, it remains to be determined whether altering the expressions of these genes could be use to modify seed coat color without causing unintended effects on alternative biochemical pathways.

Prior to the present study, currently known soybean genes did not map between markers A668_1 and K387_1 on chromosome 9 of the soybean physical genomic map, where the $\mathrm{R}$ locus is located, leaving no obvious candidates for the R locus gene (Yang et al., 2010). In our study, 14 genes identified to be differentially regulated in the black (iRT) soybean seed coat transcriptome were found to be located on chromosome 9 of the soybean genome sequence Glyma1 (Figure 4.5). Among the gene families represented, only WD40 and MYB have known roles in flavonoid regulation. However the genes demonstrated did not cluster with known flavonoid regulators by 
phylogenetic analysis (not shown). A Mob1/phocein gene was commonly upregulated in the black (iRT) soybean seed coat and Arabidopsis seedlings overexpressing PAP1 (Tohge et al., 2005). Other regulatory gene families represented on chromosome 9 were AP2/ERF, serine/threonine protein kinase, calcium/calmodulin-dependent protein kinase, and ethylene-responsive elementbinding protein. Interestingly, all upregulated and downregulated genes on chromosome 9 were located in $5.16 \mathrm{Mb}$ region that includes the nearest sequence markers to the R locus. Only the downregulated AP2/ERF transcription factor, the serine carboxypeptidase gene, and the gene moderately similar to the A. thaliana polyamine oxidase ATPAO1 were located between these sequence markers (Figure 4.5). As a result our study provides a short list of gene candidates that may serve as the focus of future efforts to identify the $R$ locus gene.

\subsection{Conclusion}

Metabolite composition and gene expression differences between black (iRT) and brown (ir T) seed coats are far more extensive than previously thought. Putative anthocyanin, proanthocyanidin, (iso)flavonoid, and phenylpropanoid isogenes were differentially-expressed between black (iRT) and brown (irT) seed coats, and UGT78K2 and OMT5 were validated to code UDP-glycose:flavonoid3-O-glycosyltransferase and anthocyanin 3'-O-methyltransferase proteins in vitro, respectively. Duplicate gene copies for several enzymes were overexpressed in 
the black (iRT) seed coat suggesting that more than one isogene may have to be silenced to engineer seed coat color using RNA interference. 


\section{CHAPTER 5}

\section{Biochemical analyses to understand red-brown soybean seed coat color and the identification of proanthocyanidin genes}

\section{Adapted from:}

Kovinich $\mathrm{N}^{1}$, Saleem $\mathrm{A}^{3}$, Arnason $\mathrm{JT}^{2}$, and Miki $\mathrm{B}^{2}$. Submitted. Identification of two anthocyanidin reductase genes and three red-brown soybean accessions with reduced ANTHOCYANIDIN REDUCTASE1 mRNA, activity, and proanthocyanidin amounts. Plant Physiology and Biochemistry.

\footnotetext{
${ }^{1}$ Designed and conducted research, analyzed data, and wrote the manuscript for publication

${ }^{2}$ Obtained funding for the research and revised the manuscript

${ }^{3}$ Developed the HPLC-DAD method
}

\subsection{Introduction}

Flavonoids are plant secondary metabolites that have a variety of ecophysiological functions including inter-organism signalling, pathogen defense, and protection from abiotic stresses Winkel-Shirley, 2001; Gould, 2004;

Grotewold, 2006). Furthermore, metabolic engineering of flavonoids has become an important target for plant biotechnology as they provide agronomic traits to crops, health benefits to foods, and may be used to color commercial genetically modified materials to facilitate their identification and monitoring (Dixon, 2005; Butelli et al., 2008; Kovinich et al., 2011a).

In soybean (Glycine max (L.) Merr.), flavonoids including proanthocyanidin (PA, a.k.a. condensed tannin) and anthocyanin pigments can be synthesized in the seed coat (Figure 5.1). Six seed coat colors have been identified to associate 


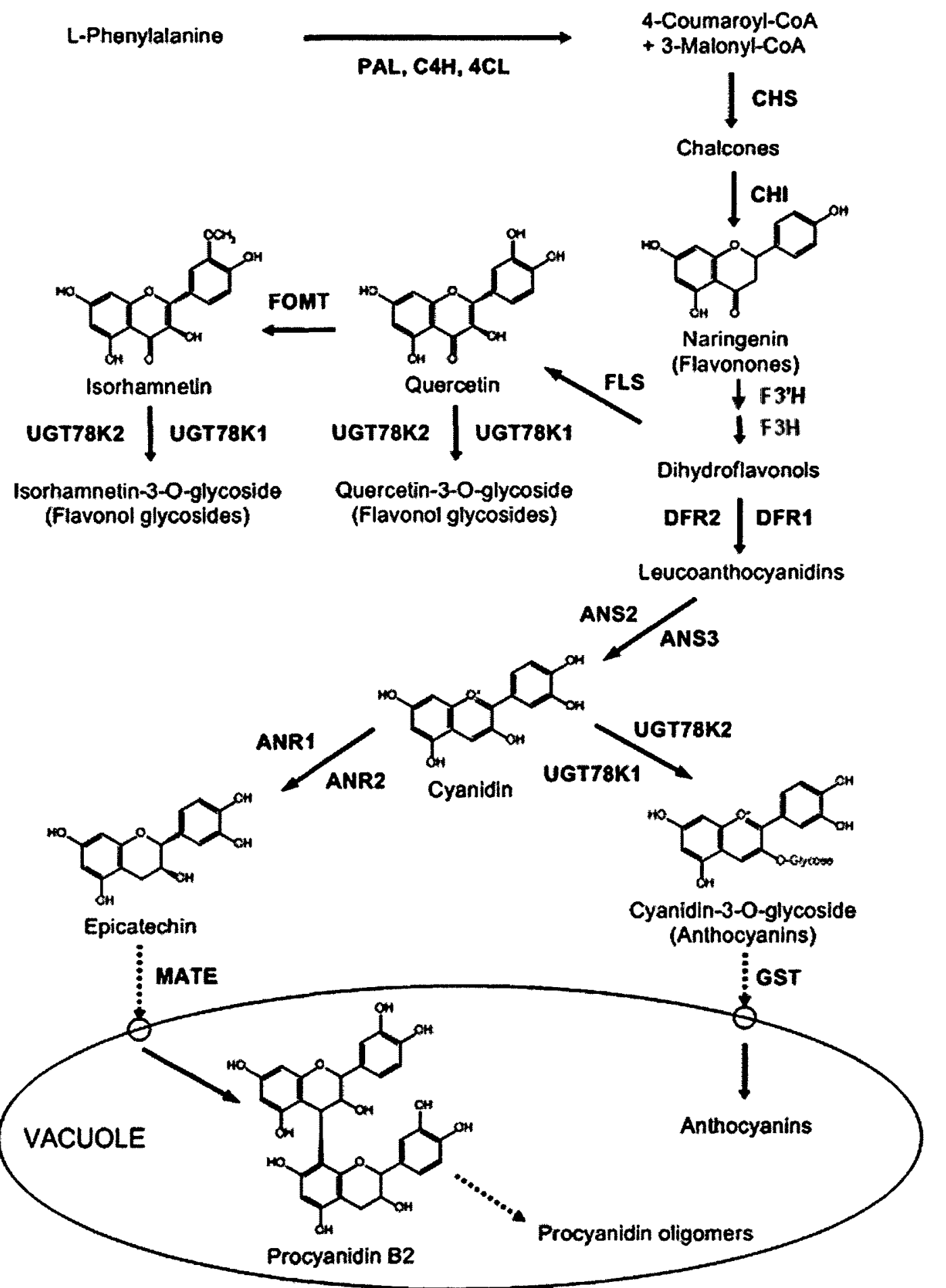

Figure 5.1. Flavonoid biosynthesis in the soybean seed coat. 
with spontaneous mutations in genetic loci by classical genetics $(I, R, T, W p, W 1$, and O) (Bernard and Weiss, 1973). The presence or absence of flavonoid-based color in the seed coat has been associated with four alleles of the llocus $\left(I, l^{\prime}, I^{k}\right.$, 1); the I allele results in the absence of pigmentation in the seed coat, giving a yellow seed due to the color of the underlying embryo, the $l^{i}$ and $l^{k}$ alleles restrict pigmentation to the hilum and to a saddle-shaped region, respectively, and the $i$ allele results in a fully pigmented seed coat (Bernard and Weiss, 1973). Mutations $I \rightarrow i\left(\right.$ or $I^{i} \rightarrow I$ ) have been associated with deletions of CHS4 or CHS1 promoter sequences and result in the abolishment of an RNA interference mechanism that silences chalcone synthase genes (CHS) specifically in the seed coat, allowing the accumulation of $\mathrm{CHS7}$ and $\mathrm{CHS} 8$ transcripts (Todd and Vodkin, 1996; Tuteja et al., 2004).

Black ( $i R T$ ) or brown (irT) soybean lines that differ in alleles of the $R$ locus differentially accumulate specific anthocyanins, PAs, isoflavones, and phenylpropanoids in the seed coat (Kovinich et al., 2011b). Alleles of the $R$ locus are associated with the differential expressions of 20 flavonoid/phenylpropanoid isogenes (Kovinich et al., 2011b), suggesting that $R$ encodes a regulatory gene.

Black (iRT) and imperfect black (iRt) seed coat color is associated with different alleles of the $T$ locus. The $T$ locus is pleiotropic controlling the type and abundance of flavonoid pigments in the seed coat and trichomes in addition to seed coat structural integrity (Woodworth, 1921; Buzzell et al., 1987; Todd and Vodkin, 1993). The recessive $t$ allele for tawny pubescence is associated with 
genetic polymorphisms that affect the function or expressions of the flavonoid 3'hydroxylase (F3'H1) gene (Toda et al., 2002; Zabala and Vodkin, 2003).

Imperfect black (W1) and buff (w1) seed coat colors in an iRt background are associated with alleles of the W1 locus, which also controls flower color (Bernard and Weiss, 1973). A 65-bp insertion in the flavonoid 3',5'-hydroxylase (F3'5'H) gene gives white (w1) flower color, while an uninterrupted $F 3^{\prime} 5^{\prime} H$ sequence is associated with purple (W1) flower color (Zabala and Vodkin, 2007).

Pigmented soybeans carrying the $W p$ allele are black (iRTWp), whereas wp gives a lighter grayish (iRTwp) grain color (Zabala and Vodkin, 2005). Microarray analysis demonstrated that high levels of transcripts of the flavonone 3hydroxylase gene $(F 3 H 1)$ were associated with purple $(W p)$ flower color, and low levels with pink (wp) color (Zabala and Vodkin, 2005).

Brown $(O)$ and red-brown (o) grain color can be controlled by the $O$ locus in an irT background (Bernard and Weiss, 1973). Anthocyanidin reductase (ANR) catalyzes the first committed biochemical step to PA biosynthesis in Arabidopsis thaliana and Medicago truncatula (Xie et al., 2003). Recently, a partial soybean sequence with $86 \%$ sequence identity to the $M$. Truncatula ANR gene mapped to the genomic region coding the $O$ locus (Yang et al., 2010). However, the function of the putative ANR sequence was not investigated, nor was it determined whether there existed biochemical differences relating to PA biosynthesis in the brown $(O)$ and red-brown (o) seed coats. In Arabidopsis, knock-out mutations in the ANR (a.k.a. BANYULS) gene result in the absence of PAs in the seed coat, 
the temporary accumulation of cyanic pigments during early seed development, and a transparent testa (tt) of the dried grain (Albert et al., 1997).

Coloring soybeans with endogenous pigments such as anthocyanins has been proposed as a strategy to enable effective detection and monitoring of adventitious presence of genetically modified (GM) soybeans in non-GM grain shipments (Kovinich et al., 2011a). Recent studies aimed at elucidating the molecular mechanisms that control soybean seed coat color have identified latestage pigmentation genes and biochemical differences associated with grain color. A UDP-glucose:flavonoid-3-O-glucosyltransferase (UF3GT; EC 2.4.1.115) gene (UGT78K1) was isolated from black soybean seed coat CDNA using homology-based methods, and its function was confirmed in vitro and by complementation of the Arabidopsis mutation ugt78k2 (Kovinich et al., 2010). UGT78K1, a second UF3GT gene (UGT78K2), and anthocyanidin 3'-Omethyltransferase (AOMT) gene (OMT5) were found to be upregulated with anthocyanin biosynthesis in the seed coat of black soybean during seed development and to have low expression levels in brown soybean (Kovinich et al., 2011b). However, genetic and biochemical differences underlying brown versus red-brown grain color remained unexamined.

Here we employed non-targeted and targeted approaches to identify differences in metabolite compositions between brown and red-brown soybean seed coats. Liquid chromatography tandem mass spectrometry (LC-MS/MS) in multiple reaction monitoring (MRM) mode revealed quantitative differences in flavan-3-ol, procyanidin, and other flavonoids. We also identified two ANR genes 
(ANR1 and ANR2) from soybean seed coat tissue and compared their expressions and activity in relation to seed coat color. We show that the three publicly available red-brown soybean varieties exhibit alterations in seed coat PA content, ANR1 transcript profiles and/or recombinant enzyme activity. Based on our results, we suggest that the manipulation of ANR1 gene expressions or activity may be a rationale approach for engineering red-brown soybean color, which would ultimately enable the visual identification of GM grains.

\subsection{Materials and Methods}

\subsubsection{Chemicals}

Cyanidin was purchased from Indofine (Somerville, NJ, USA), NADPH from Fisher Scientific (Ottawa, ON, CAN), (-)-epicatechin, procyanidin B2, cyanidin 3O-galactoside, and the 3-O-glucosides of cyanidin, quercetin and isorhamnetin were purchased from Extrasynthese (Lyon, FR). All chemicals were of the highest available purity.

5.2.2. Plant materials, growth conditions, and nucleic acid isolation Glycine $\max (\mathrm{L}$.) Merr.) varieties M100 and M11 were obtained from the Agriculture and Agri-Food Canada Soybean EMS-mutagenesis collection 2005 (M. Morrison, Eastern Ontario Oilseed Research Centre, Ottawa), varieties Clark (ir; PI547438), Soysota (r17; PI 548417), Kuro Daizu (KD; PI 81041 -1) and Agate (Ag; PI 548296) were obtained from the U.S. Department of Agriculture 
Soybean Germplasm Collection (Agricultural Research Service, University of Illinois at Champaign-Urbana). Seeds were germinated in vermiculite and transferred to soil after one week. Plants were grown in a Conviron E15 cabinet under $16 / 8 \mathrm{~h}$ light/dark at $25^{\circ} \mathrm{C} / 20^{\circ} \mathrm{C}$ for 1 month, following $12 / 12 \mathrm{~h}$ light/dark at $25^{\circ} \mathrm{C} / 20^{\circ} \mathrm{C}$ to promote reproductive development. Pods were harvested into crushed ice. Seed coats were dissected from pod and embryo material, immediately frozen in liquid nitrogen, lyophilized, and stored at $-80^{\circ} \mathrm{C}$. DNA was isolated from trifoliate unexpanded leaves using a kit (QIAGEN). RNA was isolated from seed coats as described (Wang and Vodkin, 1994), then washed using the RNeasy Plant Mini Kit (QIAGEN) to remove impurities.

\subsubsection{Metabolite analysis and LC-MS/MS}

For measurement of total soluble and insoluble PAs, lyophilized seed coats (400 - $500 \mathrm{mg} \mathrm{FSW}$ ) were ground to a fine powder in liquid nitrogen, extracted (10 $\mathrm{mg} \mathrm{mL}^{-1}$ ) with $70 \%$ acetone $(\mathrm{v} / \mathrm{v}), 0.1 \%$ ascorbic acid $(\mathrm{w} / \mathrm{v})$ in HPLC grade water, and analyzed as described previously (Li et al., 1996). For compound identification, $10-12 \mathrm{mg}$ lyophilized seed coats were extracted $\left(20 \mu \mathrm{Lg}^{-1}\right)$ with $\mathrm{MeOH}: \mathrm{H}_{2} \mathrm{O}: \mathrm{HCO}_{2} \mathrm{H}(80: 15: 5, \mathrm{v} / \mathrm{v})$ on ice with shaking $(250 \mathrm{rpm})$ for $16 \mathrm{~h}$. The supernatant was filtered through Teflon $(0.2 \mu \mathrm{m}$; Chromatographic Specialties) and $5 \mu \mathrm{L}$ aliquots were analyzed by LC-MS/MS in scanning mode as described previously (Kovinich et al., 2011b). For compound quantification, $2 \mu \mathrm{L}$ aliquots were analyzed by LC-MS/MS in multiple reaction monitoring (MRM) mode in positive polarity at unit resolution. The system consisted of a 1200 series 
Agilent liquid chromatograph with high performance autosampler (model G1376B), binary pump (model G1312A), column thermostat (model G1316A), and Peek $0.12 \mathrm{~mm}$ ID lines from column inlet onwards till tri-way splitter of a 3200 QTRAP with Peek $0.17 \mathrm{~mm}$ ID x $60 \mathrm{~cm}$ lines on splitter outlet.

For MRM, two Q1 (parent) and Q3 (product) transitions were developed per authentic standard. The most sensitive transition was used for quantification and the second for authentication of identity. For quantitative optimization, authentic standards (10-20 $\mu \mathrm{g} \mathrm{mL}^{-1}$ dissolved in methanol) were infused into the turbo ion source using an electrospary probe at a flow rate of $10 \mu \mathrm{g} \mathrm{mL}^{-1}$. Flow Injection Analysis (FIA) was applied to optimize source conditions. LC conditions consisted of a linear gradient of $32-70 \% \mathrm{MeOH}$ in $5 \% \mathrm{HCO}_{2} \mathrm{H}$ over 22 min at a flow rate of $1 \mathrm{~mL} \mathrm{~min}{ }^{-1}$. The column (Luna C18 (2), 150x $4.6 \mathrm{~mm}, 5 \mu \mathrm{m}$ particle size, (Phenomenex Inc., CA, USA) was washed by ramping up the $\mathrm{MeOH} \%$ from $70-100 \%$ for $8 \mathrm{~min}$. The initial conditions were brought back in $0.1 \mathrm{~min}$ and the column was equilibrated for 6 min before next injection.

\subsubsection{Cloning ANR1 and ANR2 cDNAs}

By searching the GenBank Glycine max EST collection using the BLASTn algorithm and the Lotus corniculatus ANR1-1 sequence as a query, forward and reverse gene-specific primers (AEV1F, AEV1R, AB4F, AEB4R) were designed to amplify partial ANR sequences from seed coat CDNA using 5' and 3' RACE ( $\mathrm{CLONTECH}, \mathrm{CA}, \mathrm{USA})$, respectively. The resulting partial cDNA fragments were cloned into the TOPO-TA vector (Invitrogen, ON, Canada) and sequenced. The 
full-length ANR1 coding region was then amplified from seed coat cDNA by endto-end PCR using primers AHF/AHR and the Pfx50 DNA polymerase (Invitrogen, ON, Canada). The ANR2 cDNA was similarly cloned using primers designed based on the Glyma08g06640 sequence. The resulting 1108 bp (ANR1) and $1017 \mathrm{bp}($ ANR2) amplicons were cloned into the Ndel and BamHI sites of the pET-14b vector (Novagen, ON, Canada) and sequenced to confirm their identities. Primers for this study are listed in Appendix M.

\subsubsection{Cloning ANR1 genes}

ANR1 sequences were amplified from genomic DNA by PCR using primers OGF/OGR and the Pfx50 DNA polymerase (Invitrogen), cloned into the BamHI and EcoRI sites of the pUC19 vector, and 5 clones per variety were sequenced.

\subsubsection{Phylogenetic analyses}

A multiple alignment of the deduced amino acid sequences was performed using MAFFT (Katoh et al., 2009). Node support was estimated by parsimony bootstrap analysis (1000 bootstrap replicates, 10 random addition sequences per bootstrap replicate with tree bisection-reconnection branch swapping, limited to a maximum of 10000 trees) using PAUP 4.0b10 (Sinauer Associates, MA, USA).

\subsection{7. $R T-P C R$ and $q R T-P C R$}

RT-PCR and qRT-PCR were performed essentially as described previously (Kovinich et al., 2011b). Briefly, for RT-PCR, RNA samples $(3 \mu \mathrm{g})$ were treated 
with DNase I (Amplification grade, Invitrogen) and first-strand cDNA was synthesized using SuperScript III Reverse Transcriptase (Invitrogen) according to the manufacturer's instructions. For quantitative RT-PCR (qRT-PCR), cDNA template was prepared as described above and reactions $(25 \mu \mathrm{l})$ consisted of 2 $\mu$ l of first-strand cDNA (or untreated RNA), $250 \mathrm{nM}$ of forward and reverse primers, and $12.5 \mu \mathrm{l}$ of the iQ SYBR Green Supermix (BioRad, ON, Canada). qRT-PCR of each target gene for each seed coat sample was performed in triplicate on cDNA samples or untreated RNA samples. qRT-PCR data and PCR efficiencies were analyzed using the Opticon Monitor 3 software (BioRad, ON, Canada). To verify the specificity of the RT-PCR reactions, melting curves were performed subsequent to each reaction in addition to fractionation of RT-PCR products on agarose gels. RT-PCR and qRT-PCR experiments were performed in triplicate. Primers used in this study can be found in Appendix M.

\subsubsection{Recombinant protein expression in Escherichia coli}

The full-length ANR1 and ANR2 coding regions were fused in-frame with the $\mathrm{pET}-14 \mathrm{~b}$ vector (above), transformed into the expression host $E$. coli BL21(DE3) pLysS (Novagen), and single colonies were selected for production of recombinant proteins. Growth, expression, and purification of soluble recombinant proteins by ion metal-affinity chromatography were performed essentially as described (Kovinich et al., 2010) with the exception that $1 \mathrm{~L}$ of $E$. Coli were routinely cultured to have enough recombinant protein for kinetics analysis. To confirm the purity of the recombinant enzyme the eluted fractions 
were visualized on $12.5 \%$ acrylamide gel stained with $0.25 \%$ Coomassie blue. The amount of purified recombinant enzyme was determined using a protein assay kit (Bio-Rad Laboratories Inc.; http://www.bio-rad.com).

\subsubsection{Recombinant enzyme assays and kinetics}

To measure recombinant enzyme activity for cyanidin, the concentrations of cyanidin were varied from 3 to $400 \mu \mathrm{M}$, and $2 \mathrm{mM}$ NADPH was used as a hydride donor. Enzyme assays (total volume $500 \mu \mathrm{l}$ ) consisted of $75 \mu \mathrm{g}$ of recombinant enzyme, cyanidin and $2 \mathrm{mM}$ NADPH in buffer ( $50 \mathrm{mM}$ MES $\mathrm{pH}$ ) and were incubated at $45^{\circ} \mathrm{C}$ for $60 \mathrm{~min}$. Gentle agitation (100 rpm) and occasional vortexing (15 - 20 min invtervals) were provided to minimize precipitation. The amount of each reaction product was determined by comparison of integrated peak areas of epicatechin to the corresponding standard curve analyzed on the same day. Standards were processed in the same manner as enzyme reactions with the exception that the recombinant enzyme was boiled for 10 min prior to incubation. Standard curves were verified to be linear $(R 2>0.99)$ over the range investigated using Chem32 software (Agilent Technologies Inc., Montreal QC, Canada). All reaction solutions were brought to $45^{\circ} \mathrm{C}$ prior to the addition of cyanidin. Cyanidin stocks were $10 \mathrm{mM}$ in $\mathrm{MeOH}$ and stored at $-80^{\circ} \mathrm{C}$. Reactions were stopped by vortexing in EtOAc $(1 \mathrm{~mL})$ for $60 \mathrm{~s}$, followed by removal of the organic phase, which was then evaporated under a stream of nitrogen gas. The residue was resuspended in $100 \mu$ of LC-MS grade $\mathrm{MeOH}$. All reactions were 
filtered through Teflon ( $0.2 \mu \mathrm{m}$; Chromatographic Specialties) and $20 \mu \mathrm{l}$ injections were analyzed by HPLC-DAD as described previously (Kovinich et al., 2010).

\subsection{Results}

5.3.1. The seed coats of three red-brown soybean varieties have reduced amounts of soluble proanthocyanidins (PAs) compared to brown soybean In Arabidopsis, knock-out mutations of the anthocyanidin reductase (ANR, a.k.a. BANYULS) gene result in the loss of proanthocyanidins (PAs) in the seed coat and the temporary accumulation of cyanic pigments (Devic et al., 1999). As the red-brown soybean also exhibits a cyanic grain color phenotype, we wanted to determine whether this may be associated with reduced PA amounts compared to brown soybean. The total amounts of soluble and solvent-insoluble PAs were measured from the seed coats of 3 brown, 2 red-brown, and 1 red-brown saddle variety (listed in Table 5.1) using previously described methods (Li et al., 1996). The 3 red-brown varieties that were available from public collections had similar seed coat luster (shiny), seed size (large), and relative timing of seed maturation (early) as the brown variety M100 (Table 5.1). However, brown soybean seed color phenotypes range from dark- to light-brown. To better represent the range of brown seed coat color intensities, the light-brown variety Clark (ir) and midbrown variety Soysota ( $\mathrm{r} 17)$ were selected to serve as comparators to the redbrown varieties, in addition to M100 which has a dark-brown seed coat. Varieties Kuro Daizu (KD) and M11 (a line produced from EMS mutagenesis of Linia $\mathrm{N} 145$ ) had red-brown color distributed across the entire seed coat surface, 
Table 5.1. Soybean lines, alleles, and phenotypic properties of their seeds.

\begin{tabular}{|c|c|c|c|c|c|c|c|}
\hline Variety & $\begin{array}{l}\text { Abbrevi } \\
\text { ated } \\
\text { Name }\end{array}$ & Alleles $^{1}$ & $\begin{array}{l}\text { Seed } \\
\text { Coat } \\
\text { Color }\end{array}$ & $\begin{array}{l}\text { Seed } \\
\text { Coat } \\
\text { Luster }\end{array}$ & $\begin{array}{l}\text { Seed } \\
\text { Size }\end{array}$ & $\begin{array}{l}\text { Relative } \\
\text { Timing of } \\
\text { Seed } \\
\text { Maturation }\end{array}$ & Source/Origin \\
\hline Clark & ir & irTO & $\begin{array}{l}\text { Light } \\
\text { brown }\end{array}$ & Dull & Medium & Late & $\begin{array}{l}\text { i-mutation in L11, } \\
\text { found in } 1965 \text { at } \\
\text { Urbana, USA }\end{array}$ \\
\hline Soysota & $\mathrm{r} 17$ & irTO & $\begin{array}{l}\text { Mid- } \\
\text { brown }\end{array}$ & $\begin{array}{l}\text { Mod- } \\
\text { erate }\end{array}$ & Small & Mid & $\begin{array}{l}\text { Collected in } 1910 \\
\text { in Italy }\end{array}$ \\
\hline $\begin{array}{l}\text { Linia } \\
\text { N145 }\end{array}$ & M100 & Unknown & $\begin{array}{l}\text { Dark } \\
\text { brown }\end{array}$ & Shiny & Large & Early & $\begin{array}{l}\text { EMS- } \\
\text { mutagenized } \\
\text { Linia N145 (PI } \\
522192 \mathrm{~A}) \\
\text { collected in } 2005 \\
\text { at Ottawa, } \\
\text { Canada }\end{array}$ \\
\hline Agate & $\mathrm{Ag}$ & "krTo & $\begin{array}{l}\text { Red- } \\
\text { brown } \\
\text { saddle }\end{array}$ & Shiny & Large & Early & $\begin{array}{l}\text { Collected at } \\
\text { Hokkaido, Japan, } \\
\text { donated in } 1951\end{array}$ \\
\hline $\begin{array}{l}\text { Kuro } \\
\text { Daizu }\end{array}$ & KD & irTo & $\begin{array}{l}\text { Red- } \\
\text { brown }\end{array}$ & Shiny & Large & Early & $\begin{array}{l}\text { Collected in } 1929 \\
\text { at Hokkaido, } \\
\text { Japan }\end{array}$ \\
\hline $\begin{array}{l}\text { Linia } \\
\text { N145 }\end{array}$ & M11 & Unknown & $\begin{array}{l}\text { Red- } \\
\text { brown }\end{array}$ & Shiny & Large & Early & $\begin{array}{l}\text { EMS- } \\
\text { mutagenized } \\
\text { Linia N145 (PI } \\
522192 \text { A), } \\
\text { collected in } 2005 \\
\text { at Ottawa, } \\
\text { Canada }\end{array}$ \\
\hline
\end{tabular}

${ }^{1}$ All lines used in this study were homozygous for the $I, R, T$ and $O$ alleles listed. 
whereas variety Agate $(\mathrm{Ag})$ with red-brown saddle $\left(I^{k} r T o\right)$ had pigmentation confined to a saddle-shaped region that encompassed $60-71 \%$ of the seed coat surface area (not shown).

Reaction of seed coat extracts with DMACA reagent showed that red-brown varieties $\mathrm{KD}$ and $\mathrm{M} 11$ had $46-71 \%$ of the amount of soluble PAs compared to the brown varieties (Figure 5.2A). The red-brown saddle variety $\mathrm{Ag}$ had $32-45 \%$ soluble PAs compared to brown varieties (significantly less than what may be attributed to the incomplete (saddle-shaped) distribution of pigments across 60$71 \%$ of the seed coat surface area) (Figure 5.2A). By contrast, the amounts of solvent-insoluble PAs measured following acid-catalyzed hydrolysis of seed coat residues were not found to differ significantly between red-brown and brown varieties (Figure $5.2 \mathrm{~B}$ ).

PA and anthocyanin branchpoint enzymes use the same flavonoid intermediate, cyanidin, as a substrate, suggesting the potential for competition between these pathways. To investigate whether the decrease in soluble PA amounts may be associated with an increase in the levels of cyanic pigments (i.e. anthocyanidins or anthocyanins), seed coats were extracted with aqueous methanol and cyanic pigments were quantified by photospectroscopy in comparison to the commercial standard cyanidin (Figure 5.2C).

Red-brown seed coats had an increase in cyanic pigment amounts, however the increase (0.5-1.4 $\mathrm{mg} \mathrm{g}^{-1}$ lyophilized seed coat (LSC) was much less than the decrease in soluble PA amounts (15.3-50.4 $\mathrm{mg} \mathrm{g}^{-1} \mathrm{LSC}$ ) (compare Figure $5.2 \mathrm{C}$ to Figure 5.2A). 
A
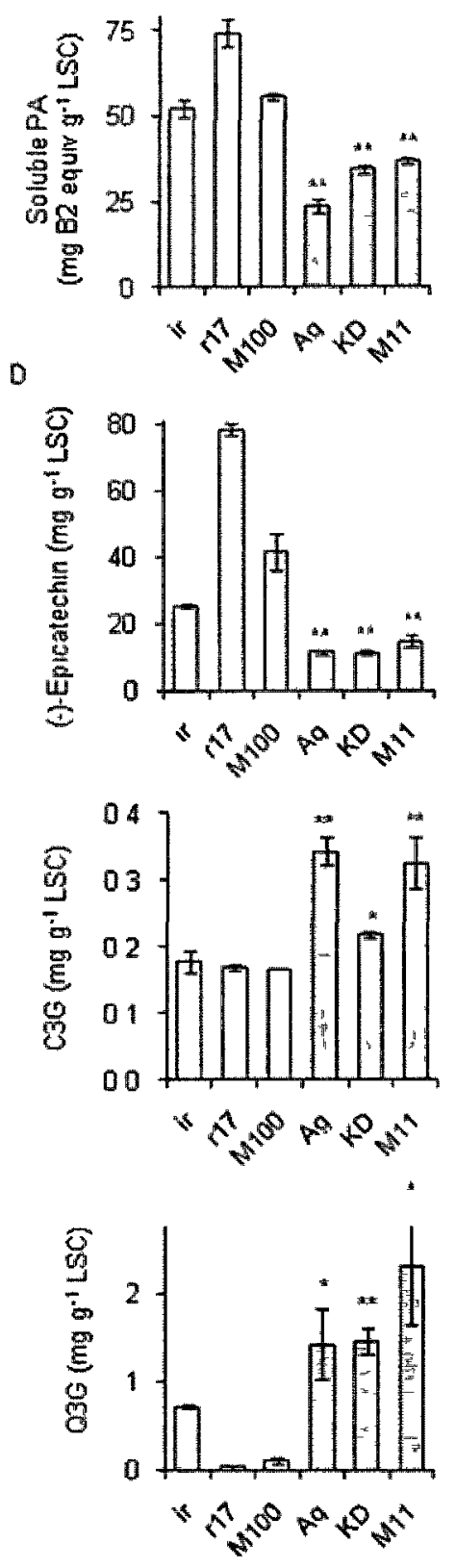

8
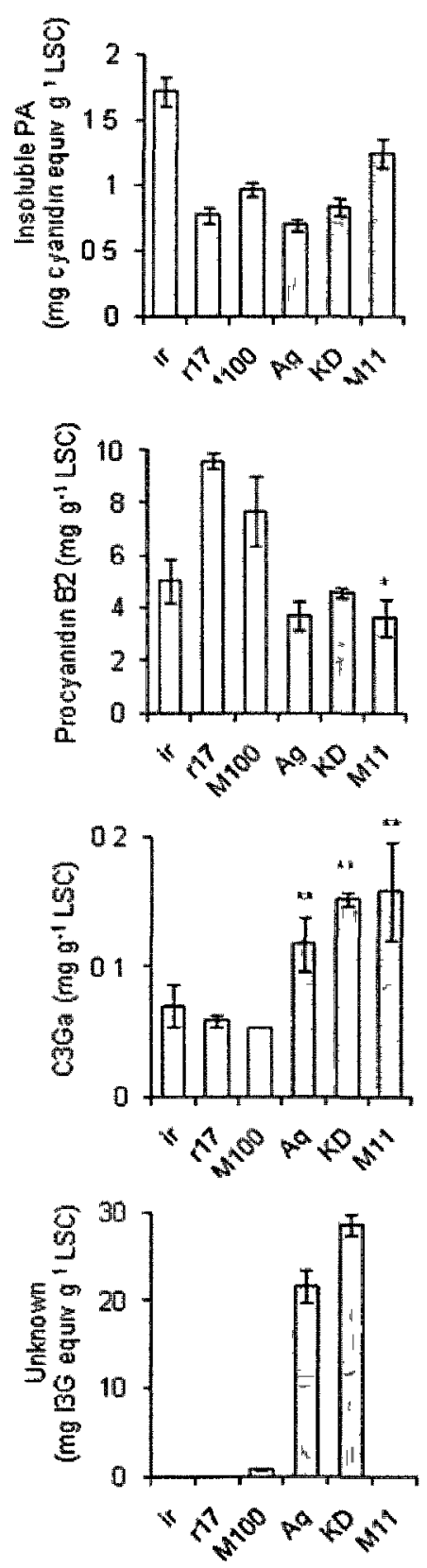
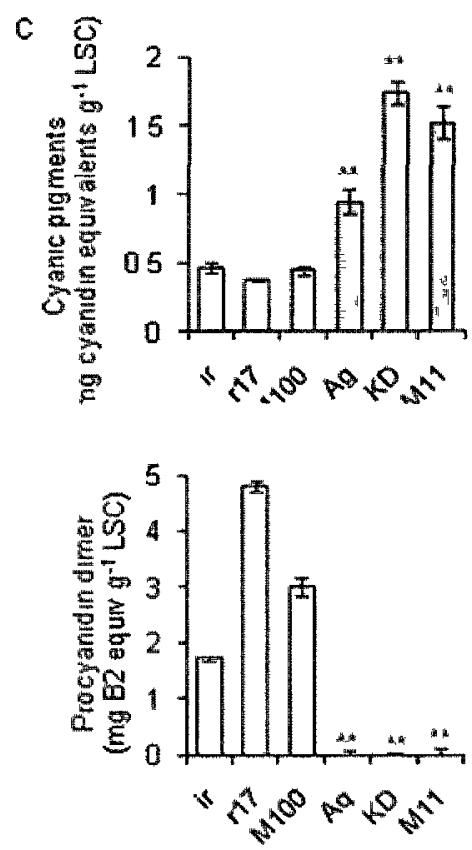

Figune 5.2. Flav onord amounts from the seed coats of brown (O) and red-brown (0) soybean vartetles expressed in units $\mathrm{mg}$ flavonoid per gram lyophilized seed coat (LSC) (A) Total soluble PAs by DMACA assay. (E) total insoluble PAs by acidcalalyzed hydrolysis, (C) cyanic pigments by photospectroscopy (Abs $520 \mathrm{~nm}$ ). (D) indwidual flav onoids by LC-MSMS in multuple reaction montionng (MRM) mode thite bars represent brown (O) soybean wanelles $(r, 177$, and $M 100)$ and grey bars represent red-brown (o) varreties ( $\mathrm{Ag}, \mathrm{KD}$, and $\mathrm{M11}$ ) Values are mean and SD from three brological replicates

To determine the identities of the differentially accumulated metabolites and to measure their amounts, we performed high performance liquid 
chromatography-tandem mass spectroscopy (HPLC-MS/MS) in scanning and multiple reaction monitoring (MRM) modes, respectively, in comparison to authentic standards. Seven flavonoids had accumulated to different amounts in red-brown seed coats compared to the brown lines (Figure 5.2D). Red-brown seed coats had reduced amounts of (-)-epicatechin, procyanidin B2 (line M11), and an unknown procyanidin dimer (Figure 5.2D top panels), elevated amounts of the anthocyanins cyanidin-3-O-glucoside (C3G) and cyanidin-3-O-galactoside (C3Ga) (Figure 5.2D middle panels) and the flavonol quercetin-3-O-glucoside (Figure 5.2D bottom left). The red-brown lines $\mathrm{Ag}$ and $\mathrm{KD}$, but not the EMS mutagenized line M11 had elevated amounts of an unknown compound that had the same $\mathrm{m} / \mathrm{z}$ (479.0) but different chromatographic retention time as isorhamnetin-3-O-glucoside (Figure 5.2D bottom right panel).

Overall, these results demonstrated that red-brown soybean varieties had major reductions in the amount of soluble PAs in their seed coat compared to the brown varieties, accompanied by minor increases in specific anthocyanin, flavonol, and unknown metabolites.

5.3.2. Identification of ANR1 and ANR2 genes from the G. max seed coat Yang et al (2010) recently mapped a putative anthocyanidin reductase (ANR) gene sequence to the genomic region coding the O locus (Weiss, 1970) and speculated that $O$ may correspond to ANR (Yang et al., 2010). We have shown that red-brown soybean seed coats have reduced amounts of soluble PAs (Figure 5.2A); further suggesting the color phenotype may be associated with a 
defect in the PA pathway. These results prompted us to identify the functional ANR gene sequence(s) from soybean to determine whether red-brown seed coat color may correspond to defects in the ANR gene(s).

As the Glyma1 soybean genome sequence (Schmutz et al., 2010); for sequence see www.phytozome.net/soybean) was not available at the time our study was initiated, G. max ESTs from the GenBank collection were searched with the tBLASTn algorithm using the sequence of every reported catalytically characterized ANR protein as a query in order to identify G. max ANR candidates (September, 2007). Two G. max ESTs (GenBank accessions EV264997 and BM092813) were identified by tBLASTn that had high identity (>80\%) to ANR1-1 from Lotus corniculatus (LCANR1-1; ABC71332). 5'- and 3'-RACE using seed coat cDNA from brown soybean variety Clark (ir) as a template was performed to extend the EST sequence information, and revealed that both ESTs coded the same cDNA, which we named ANR1 (JF433915). The full-length ANR1 cDNA was $1324 \mathrm{bp}$ and the deduced amino acid sequence had high identity (83\%), and similar length to LcANR1-1 (337 residues vs. 338 for LcANR1-1).

Primers AHF and AHR designed to amplify from the 5-coding region to the 3'UTR of the ANR1 cDNA (Appendix N) were used to amplify and clone the corresponding genomic regions from variety ir to gain insight into the structural organization of the ANR1 gene in the G. max genome. The genomic ANR1 sequence coded six exons and five introns (not shown), similar to the banyuls (BAN) gene from Arabidopsis (NC_003070). A BLASTn search of the Glyma1 soybean genome sequence using genomic $A N R 1$ as a query found it to be $100 \%$ 
identical to the predicted gene Glyma08g06630 (www.phytozome.net/soybean), and $70 \%$ identical to a second predicted gene Glyma08g06640 (76\% identical at the amino acid level), which we named ANR2 (JN098512) due to its sequence similarity.

To determine the relationship of putative G. max ANR proteins with other catalytically characterized reductase epimerase dehydratase (RED)-superfamily proteins, we performed a phylogenetic analysis. The phylogenetic tree demonstrated that ANR1 and ANR2 were most closely related to ANR proteins than to other reductase-epimerase-dehydrogenase (RED) superfamily proteins (Figure 5.3). Gene expression analysis by RT-PCR demonstrated highly divergent expression patterns; with ANR2 expressed at low levels in all organs, and ANR1 expressed preferentially and highly in the seed coat (Figure 5.4) consistent with the identification of (-)-epicatechin and (-)-epicatechin-based procyanidins in the seed coat of brown soybean (Kovinich et al., 2011b).

To confirm the putative catalytic function of ANR1 and ANR2 proteins for the reduction of cyanidin to (-)-epicatechin, their open reading frames were separately cloned from brown soybean (ir) seed coat cDNA into the pET-14b Nterminal hexahistidine fusion tag expression vector. The resultant plasmids were transformed into $B L 21(\mathrm{DE} 3)$ pLysS Escherichia coli, expressed at $16^{\circ} \mathrm{C}$ and the recombinant his-tagged proteins were purified from the soluble fraction by ionmetal-affinity chromatography (IMAC). Separation of the IMAC fractions by SDSPAGE followed by staining with Coomassie Blue showed apparently pure 


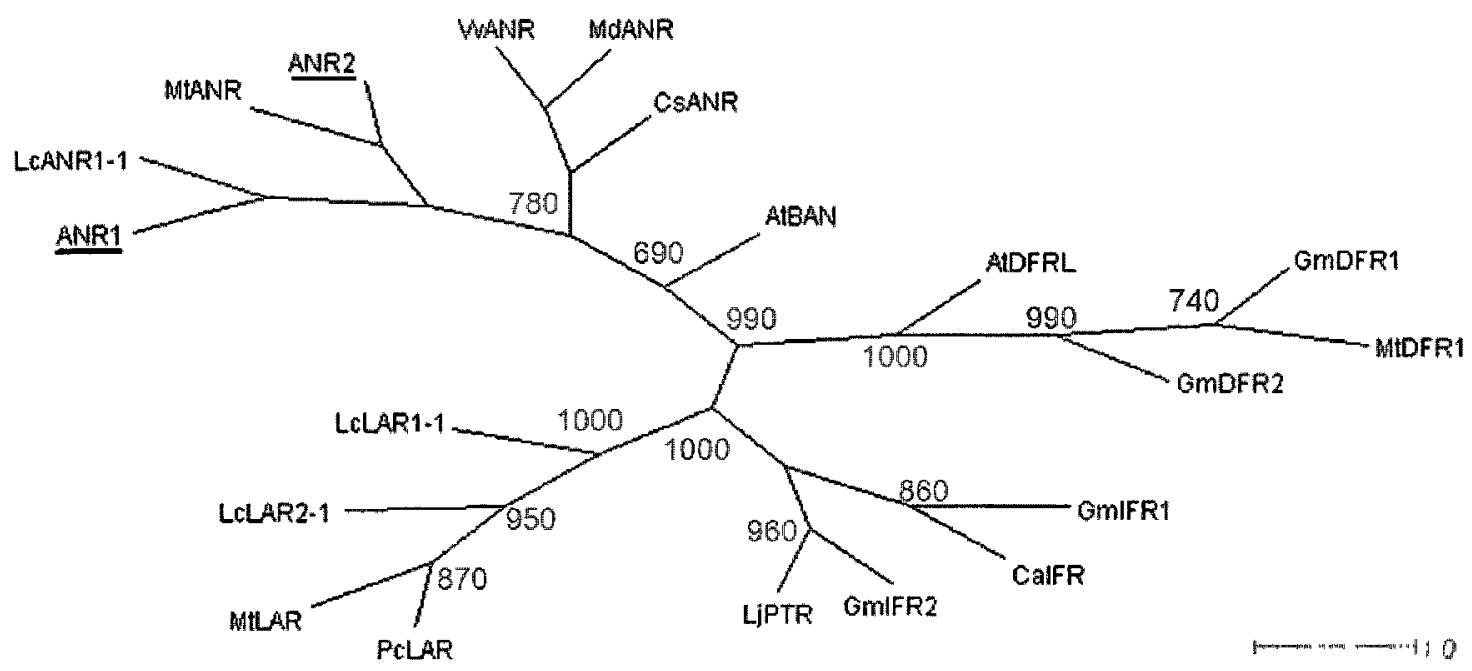

Figure 5.3. Phylogenetic tree of ANR and other reductase-epimerasedehydrogenase (RED) superfamily proteins. Amino acid sequences of characterized enzymes from Arabidopsis, AtBAN (NP_176365), AtDFRL (CAP08805); Camellia sinensis, CsANR (AAT68773); Cicer arietinum, CalFR (Q00016); G. max, ANR1, ANR2, GmDFR1 (AAD54273), GmDFR2 (ABM64803), GmIFR1 (AAF17577), GmIFR2 (AAF17578); L. corniculatus, ANR1-1 (ABC71332), LAR1-1 (ABC71324), LAR2-1 (ABC71328); L. japonicus, PTR (BAF34844); Malus $\times$ domestica, MdANR (AAX12184); M. truncatula, ANR (AAN77735), DFR1 (AAR27014), LAR (CAI56327); Phaseolus coccineus, LAR (CAI56322); and Vitis vinifera ANR (BAD89742) were aligned using MAFFT (Katoh et al., 2009) and the phylogenetic tree was produced using Dendroscope (Huson et al., 2007). The scale bar represents 1.0 substitutions per site. 


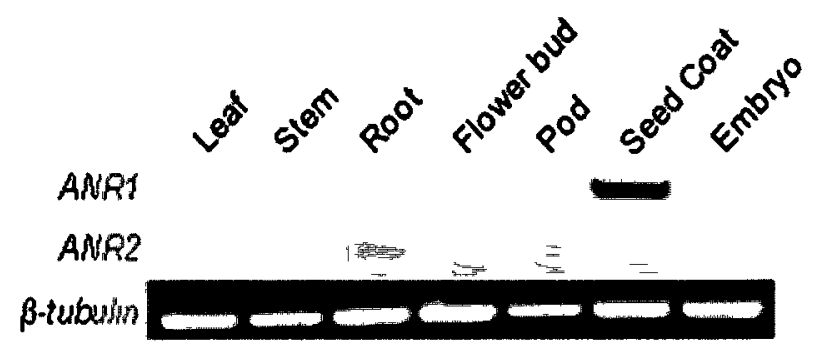

Figure 5.4. Transcript detection of ANR1 and ANR2 from Clark (ir) cDNA by RTPCR with gene-specific primers. 
recombinant enzymes with estimated molecular masses of ca. $38.7 \mathrm{kDa}$ and 38.9 kDa for ANR1 and ANR2, respectively (Figure 5.5A). These values matched well with their respective calculated molecular masses (38.72 kDa for ANR1 and $39.30 \mathrm{kDa}$ for ANR2) with the inclusion of the $2.18 \mathrm{kDa} \mathrm{N}$-terminal tag.

Recombinant enzyme assays were conducted using NADPH as a hydride donor and cyanidin as an acceptor. Incubation of ANR1 or ANR2 recombinant enzymes with cyanidin and NADPH resulted in the production of (-)-epicatechin, as identified by the identical LC retention time (Rt: $6.9 \mathrm{~min})$, parent ion $([\mathrm{M}+]$ $291.4 \mathrm{~m} / \mathrm{z}$ ) and major ion fragment(s) $(139.1 \mathrm{~m} / \mathrm{z}$ and/or $123.1 \mathrm{~m} / \mathrm{z})$ compared to the authentic standard (Figure 5.5B). The boiled enzyme and bacteria expressing the corresponding empty vector could not catalyze this reaction (not shown).

In summary, two functional ANR genes were identified from the Glyma1 soybean genome sequence that exhibit different expression patterns, with ANR2 being expressed at low levels in all organs, and ANR1 preferentially and highly expressed in the seed coat. These results suggest that ANR1 has a major role in PA biosynthesis in the soybean seed coat.

\subsubsection{Red-brown soybean varieties $M 11$ and KD have non-synonymous} substitutions in the ANR1 gene that confer minor reductions in enzyme activity

Flavan-3-ol and PA contents from the seed coat of brown soybean were shown to be (-)-epicatechin and (-)-epicatechin-based, respectively (Kovinich et al., 2011b). Figure 5.2A demonstrated that the three publically available redbrown soybean varieties have reduced amounts of soluble PAs in the seed coat 
A
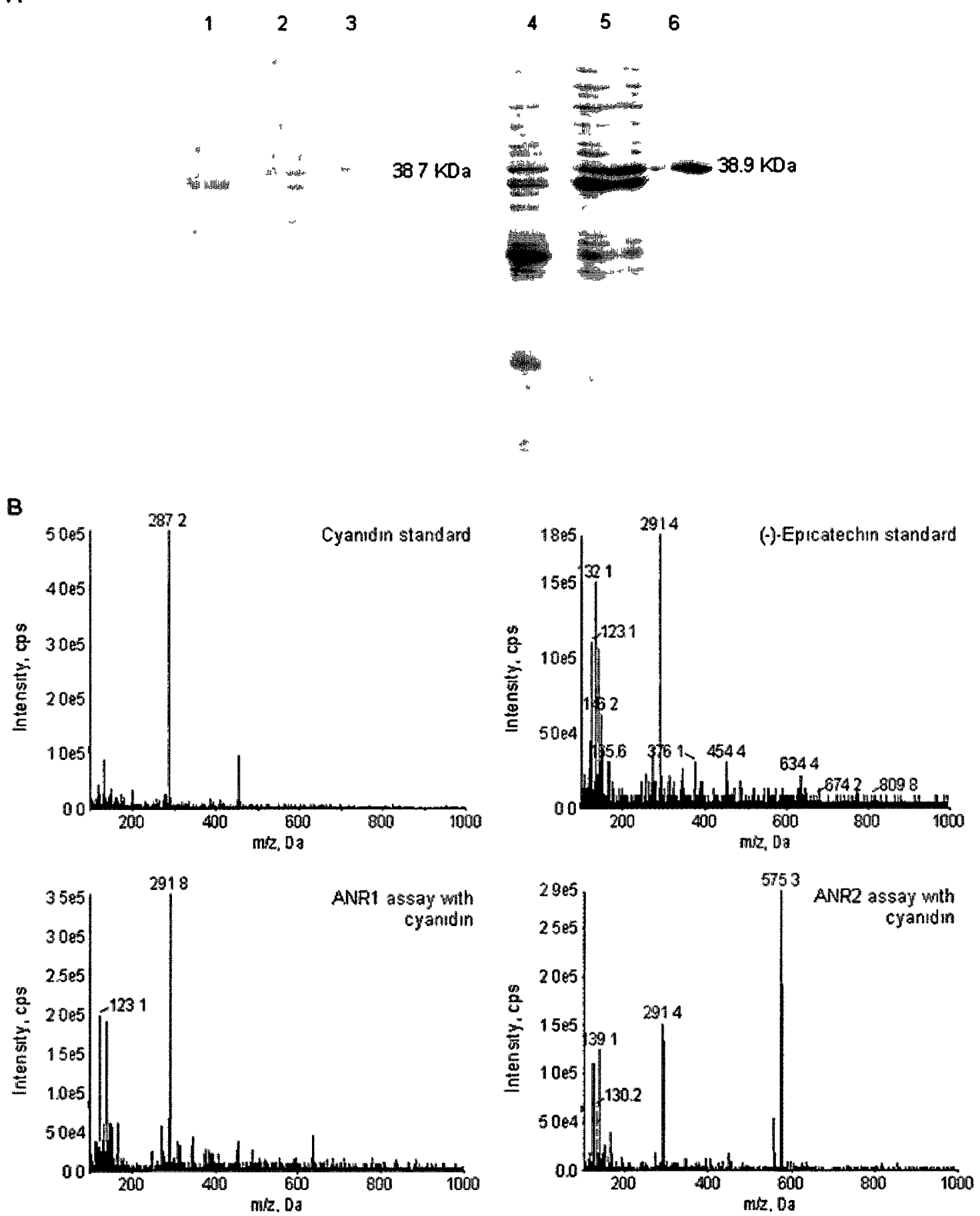

Figure 5.5. Purification of his-tagged ANR1 and ANR2 and identification of reaction products. (A) SDS-PAGE analysis of ANR1 and ANR2: total soluble protein from $E$. coli expressing ANR1 prior to induction with $100 \mu \mathrm{M}$ IPTG (lane 1), $24 \mathrm{~h}$ post-induction (lane 2); purified ANR1 (lane 3); total soluble protein from 
E. coli expressing ANR2 prior to induction with $100 \mu \mathrm{M}$ IPTG (lane 4), $24 \mathrm{~h}$ postinduction (lane 5), and purified ANR2 (lane 6). (B) ANR1 and ANR2 enzyme reactions as revealed by LC-MS/MS. LC retention times: cyanidin (Rt: $10.0 \mathrm{~min}$ ); (-)-epicatechin (Rt: 6.9). The $\mathrm{m} / \mathrm{z} 575.3$ (lower right panel) corresponded to a cyanidin (Cy) dimer formed non-enzymatically, as indicated by its presence in control (boiled enzyme) and enzyme reactions at higher Cy concentrations.

compared to brown varieties. To determine whether reduced soluble PA amounts in the red-brown seed coat may be associated with sequence-based defects in the ANR1 gene, the 3-kb genomic region coding ANR1 from variety M11 was cloned using primers OGF/OGR (Appendix N) and compared to ANR1 sequences from the 5 other varieties (Table 5.1). To ensure accurate sequence information, five individual clones per variety were sequenced with at least three times coverage.

Genomic ANR1 sequences from all brown varieties were identical, and were the same as the predicted gene Glyma08g06630 from the reference soybean genome sequence Glyma1 (www.phytozome.net/soybean). By contrast, each red-brown variety had at least one sequence polymorphism compared to the ANR1 sequence from brown genotypes (Table 5.2). Red-brown saddle variety $\mathrm{Ag}$ had a single base pair insertion in intron 1 at position 550 . Red-brown variety KD had two non-synonymous substitutions; $T$ to $C$ in exon 3 at position 1269 , and $G$ to $C$ in exon 4 at position 1957. These mutations corresponded to the predicted amino acid changes of V122A and G214A (Table 5.2) in conserved and nonconserved residues, respectively (Figure 5.6). Red-brown EMS-mutagenized variety M11 had the same $\mathrm{G}$ to $\mathrm{C}$ substitution in exon 4 at position 1957, and had 
Table 5.2. Comparison of ANR1 genomic and amino acid sequences among brown (O) and red-brown (o) soybean varieties*.

\begin{tabular}{lcccc}
\hline Variety & $\begin{array}{c}\text { Percent identity } \\
\text { to ir (genomic } \\
\text { DNA) }\end{array}$ & Mutation & Position & $\begin{array}{c}\text { Amino acid } \\
\text { substitution }\end{array}$ \\
\hline $\mathrm{Ir}$ & 100 & None & $\mathrm{N} / \mathrm{A}$ & $\mathrm{N} / \mathrm{A}$ \\
$\mathrm{r} 17$ & 100 & None & $\mathrm{N} / \mathrm{A}$ & $\mathrm{N} / \mathrm{A}$ \\
$\mathrm{M} 100$ & 100 & None & $\mathrm{N} / \mathrm{A}$ & $\mathrm{N} / \mathrm{A}$ \\
$\mathrm{Ag}$ & 99 & $\mathrm{~A}$ insertion & 550 (intron 1) & N/A \\
$\mathrm{KD}$ & 99 & $\mathrm{~T} \rightarrow \mathrm{C}$ & 1269 (exon 3) & V122A \\
& & $\mathrm{G} \rightarrow \mathrm{C}$ & 1957 (exon 4) & $\mathrm{G} 214 \mathrm{~A}$ \\
$\mathrm{M} 11$ & 99 & $\mathrm{~A} \rightarrow \mathrm{T}$ & 1870 (exon 4) & N185I \\
& & $\mathrm{G} \rightarrow \mathrm{C}$ & 1957 (exon 4) & G214A \\
\hline
\end{tabular}

*See Table 5.1 for a description of varieties. 
ANRL-II ANR1-r17 ANR $\perp-M 100$ ANR1-Ag ANR1-KD ANE1-M11

BNR1-Ir ANR1-FI 7 ENF $1-M=00$ ANEI-Ag ANR 1-KD ANR1-MII

ANR1-Ir ANE $1-r 17$ ANR $\perp-M 100$ ANR $\perp-A g$ ANE1-KD ANP1-M1

ANR1 - I r ANR 1-I17 ANR1-M100 ANR1-Ag ANR1 - KD ANE1-MI1

ANR 1-1 I ANP $1-=17$ ANRL-MI00 ANR $1-A g$ ANR $\perp$-KD MNR $\perp-M \perp 1$

ANF 1-1r ANR1- 17 ANRI-M 100 ANR1-Ag ANE 1-KD ANR1-M11
NADPE

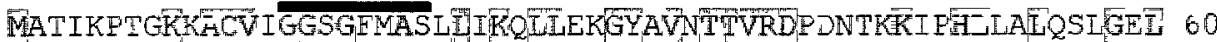

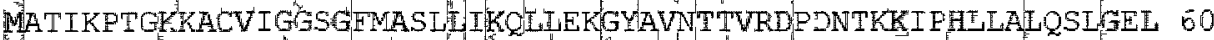

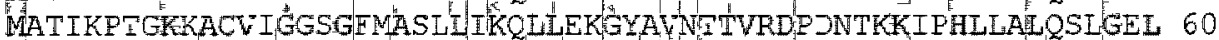

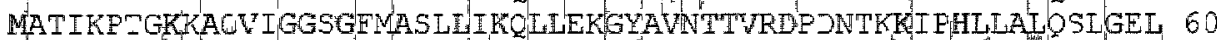

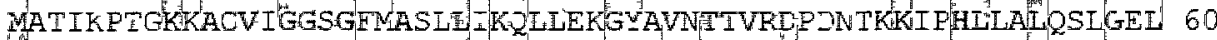

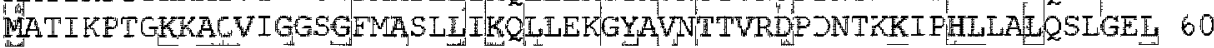

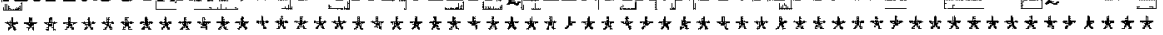

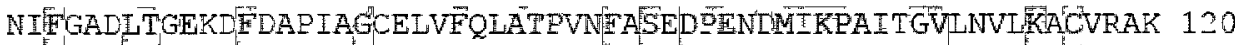

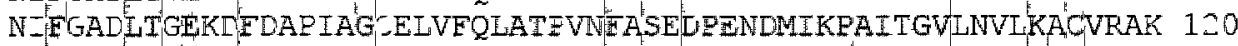
N-FGADTGEEDEAPIAG ELVEQLATPVAFASEDPENDMEKPAITGULNVLFACVRAK 120 NEFGADTGEKIPDAPIAGCELVEQEATPVN TASEDPENDM KPAITGVLNVLKALVRAK 120

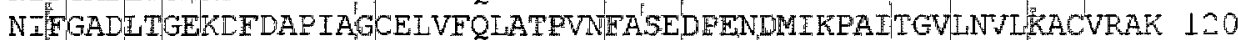

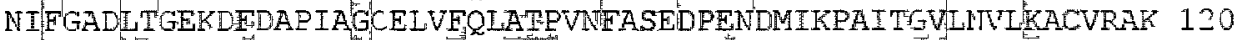

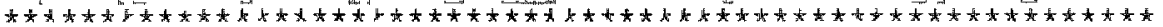

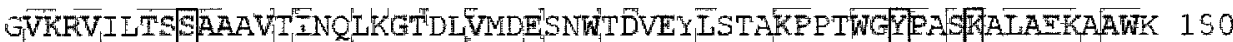

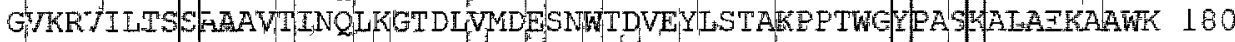

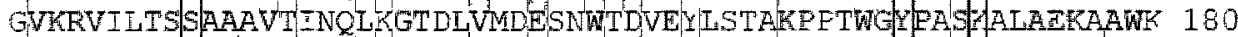

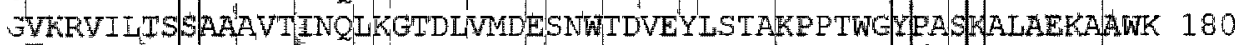

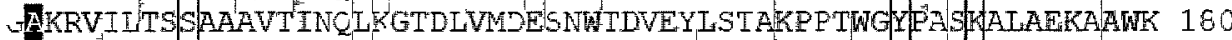

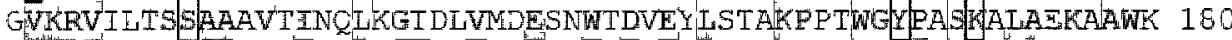

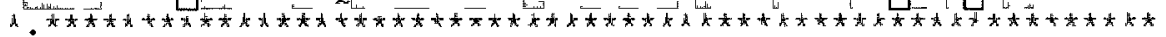

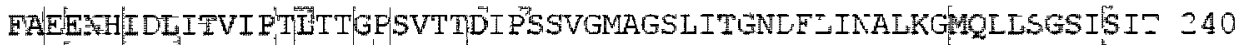

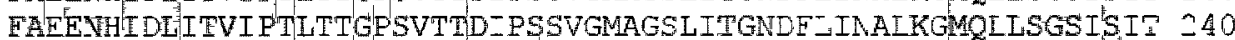

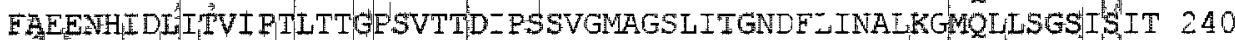
EAEENHTDLITVI PTLTTGE SVTTTI-PSSVGMAGSLITGNDF_INALKGMQLLSGSISIT 240 FAEEAHTDLITVIPTETTGPSVTTD-PSSVGMAASLITGNDFLINALKGMQLISGSISIT 240 FAEEIH DITITIPTLTTGESVTTDIPSSVGMASLITGNDELTNALKGMOLLSGSISIT 240

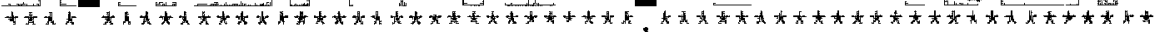

HVEDICRAOIFVAEKESASGRYICCAHNTSVPELAKFISKRYPOYKKIPTEFDDCPSKAKL 300 HVEDICRAQI IVVAEKESA SGRY ICCAHNTSVPELAKELSKRYPQYKI PTEFDDCPSKAKت 300

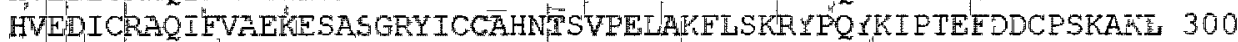
HVEPICRAOIFVA ERE SASGRYICCAHNTSVPELAKEISKRYPQYKI TTEFDDCPSKAKE 300

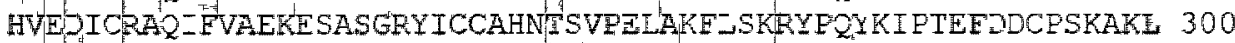
HVE ICRA O-FVAERESASGRYICCAHNFSVPEIAKFLSKRYFOYKI IEFDDCPSKAKL 300

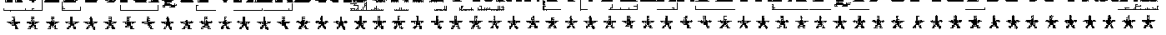

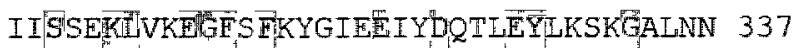

II SSEKIVKEISES FKYGIEEIYDUTLEYTKSKGALNN 337 ITSSEWCVKEGESTKYGIEFIYDOTIEYLKSKGALNN 337 I-SSEKLVKEGESTKIGIEEIYPQTLEYLKSKGALNN 337 I_SSEKEVKEGESEKYGIEZIYDOTLEYLKSKOALNN 337

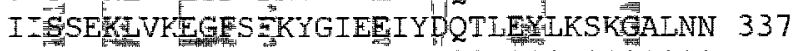

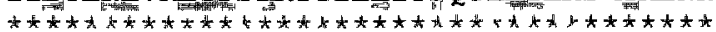

Figure 5.6. Alignment of $G$. max ANR1 proteins from brown $(O)$ seed coat varieties (ir, r17, M100) and red-brown (o) seed coat varieties (Ag, KD, and M11) using the ClustalW program with default parameters. Mutations are shown with black background. Grey background represents amino acid residues that are conserved among all catalytically validated ANR proteins: (AtBAN (NP_176365), CsANR (AAT68773), G. max ANR1-ir, LcANR1-1 (ABC71332MdANR (AAX12184); M. truncatula, ANR (AAN77735); and Vitis vinifera ANR 
(BAD89742). The putative NADPH binding site and the catalytic triad (Gomez et al., 2009) are represented by a thick black line above the sequences and boxed amino acids, respectively.

a unique substitution in exon 4 ( $A$ to $T$ at position 1870) that conferred a predicted amino acid change of $\mathrm{N} 185 \mathrm{I}$ (Table 5.2) in a conserved region (Figure $5.6)$.

To determine whether these amino acid substitutions altered enzyme activity, the respective ANR1 ORFs from varieties KD and M11 were individually cloned into an $\mathrm{N}$-terminal hexahistidine fusion tag vector for comparison of enzyme kinetics with ANR1 from brown soybean variety ir in vitro. Recombinant enzyme assays using NADPH as a hydride donor and cyanidin as an acceptor revealed minor reductions $\left(1.8-26.2 \mathrm{fmol}\right.$ epicatechin $\left.\mathrm{s} \mathrm{ug}^{-1}\right)$ in the activity of the ANR1 proteins from varieties KD and M11 compared to ANR1 from variety ir (Figure 5.7). Calculated $\mathrm{Km}$ and $\mathrm{Vmax}$ values are not given as the enzymes were intensely inhibited by cyanidin concentrations above $\sim 100 \mu \mathrm{M}$ (not shown).

These results suggest that reduced ANR1 activity could (at least in part) account for some of the reduction of soluble PAs in the red-brown seed coats of varieties $K D$ and M11 (Figure 5.2A), however additional defects in PA biosynthesis would likely be required to account for the major reductions in soluble PA amounts. 


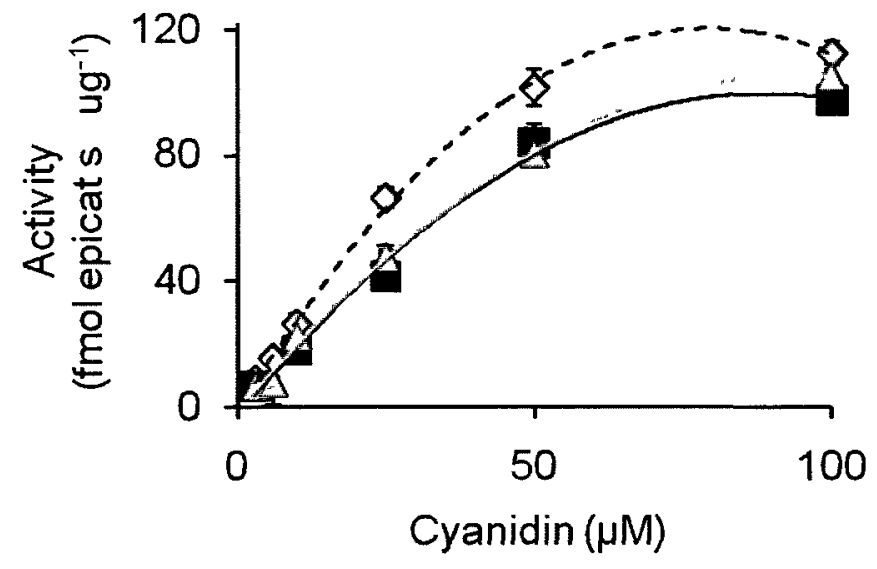

Figure 5.7. In vitro kinetics of recombinant ANR1 enzymes from brown soybean variety ir $(\diamond)$ and red-brown soybean varieties M11 ( $)$ and $K D(\Delta)$. Activity was measured as amount of epicatechin produced per second per microgram enzyme. 
5.3.4. Red-brown soybean seed coats of varieties Ag and KD have reduced ANR1 transcript levels

To determine whether reduced soluble PA amounts may be associated with transcript levels of the ANR genes, we compared ANR1 and ANR2 transcript profiles between red-brown and brown lines by quantitative RT-PCR (qRT-PCR) using seed coat cDNA as a template. The $25-50 \mathrm{mg} \mathrm{FSW}$ stage of seed development was chosen as the developmental stage for qRT-PCR analysis because ANR1 transcripts were previously shown to be at maximum levels at this stage in brown (irTO) variety Clark (ir) and its black (iRTO) isoline (Kovinich et al., 2011b). Phosphenol pyruvate carboxylase (PEPC) was used as an endogenous reference for normalization of the qRT-PCR measurements of target genes across seed coat samples, as PEPC has been used previously as a reference for gene expressions from pigmented and non-pigmented soybean seed coats, and is expressed at similar levels in a wide range of soybean tissues (Sugimoto et al., 1992; Tuteja et al., 2004; Kovinich et al., 2011b). The expressions of other genes situated at the branchpoint between PA and anthocyanin biosynthesis, (namely UGT78K2 (HM591298) coding UF3GT (Kovinich et al., 2011b), LAR1 (JF433916) putatively coding leucoanthocyanidin reductase (Kovinich et al., 2011b), and ANS2IANS3 (AY382829/AY382830) coding anthocyanidin synthases $(\mathrm{Ku}, 2009)$ were also measured to investigate for a relationship with seed coat color.

qRT-PCR analysis demonstrated that only ANR1 and ANS2IANS3 were expressed at consistently high levels among all brown varieties, with the other 
genes showing highly variant expression levels with no consistent correlation with seed color (Figure 5.8). The EMS-mutagenized variety M11 had similar ANR1 expression levels compared to the brown varieties, however out of all the genes investigated, only ANR1 expressions were significantly reduced in spontaneous red-brown mutants $\mathrm{KD}$ and $\mathrm{Ag}$ with the recessive o locus (Figure 5.8). These results suggest that reduced ANR1 gene expressions have a role in the reduction of soluble PA content in the seed coats of naturally-occurring (spontaneous) red-brown varieties $\mathrm{KD}$ and $\mathrm{Ag}$ with the recessive o locus. 

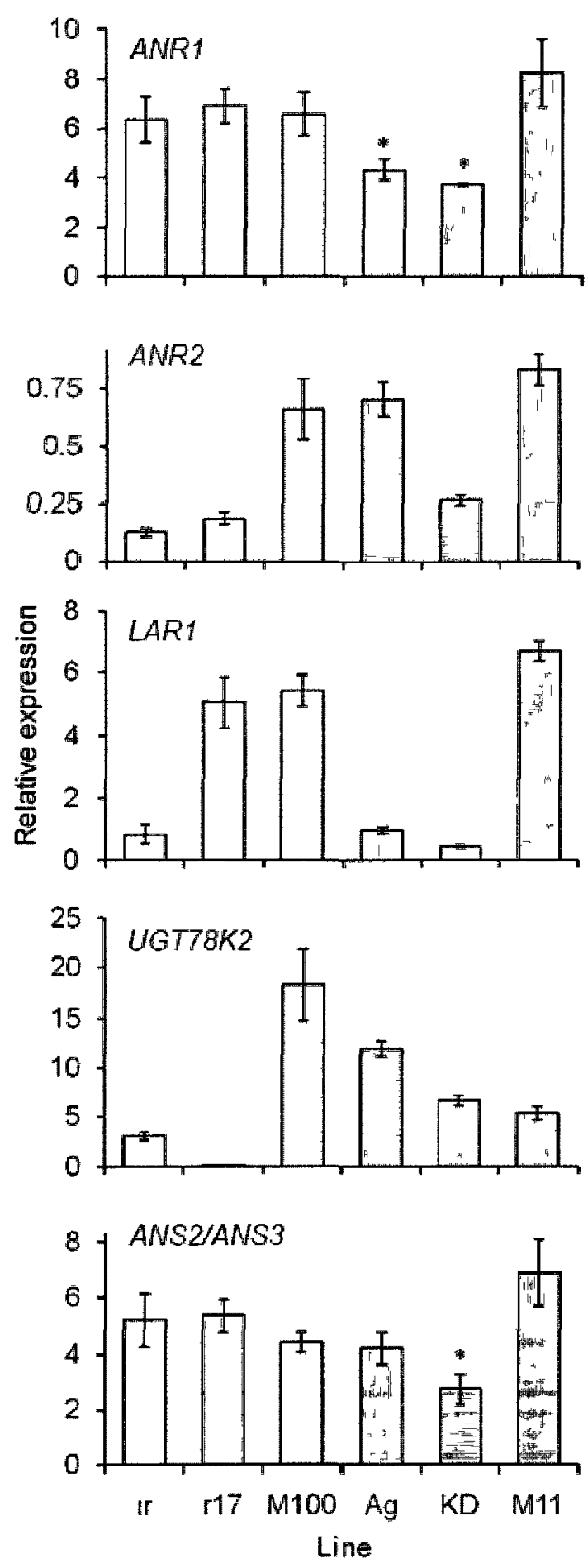

Figure 5.8. Seed coat transcript profiles of $P A$ and anthocyanin branchpoint genes measured by qRT-PCR. PA genes ANR1, ANR2 and LAR1, anthocyanin genes UGT78K2 and ANS2/ANS3. Student's $t$ test significant at $P<0.05\left(^{*}\right)$. 


\subsection{Discussion}

The seed coats of yellow $(I)$ soybean have reduced amounts of flavonoids compared to those of pigmented ( $I$ ) genotypes due to differences in the expressions of chalcone synthase genes mediated by a post-transcriptional gene silencing mechanism (Todd and Vodkin, 1996; Tuteja et al., 2004). The seed coats of brown ( $r$ ) soybean have reduced amounts of specific anthocyanins, PAs, isoflavones, and phenylpropanoids compared to black $(R)$ soybean, due to differences in the expressions of a putative regulatory gene (Kovinich et al., 2011b). Here we have shown that three red-brown soybean accessions have major reductions in the amounts of soluble PAs compared to brown soybean lines (Figure 5.2A). A previous study demonstrated that red faba beans (Vicia faba) have reduced PA content compared to the brown variety with normal flower color (Martin et al., 1995). Similarly, red to orange-brown and dark-red varieties of cowpea (Vigna unguiculata) had reduced PA content compared to their beige comparators (Price et al., 1980). These results have significant implications for the metabolic engineering of seed color for the purposes of marking commercial transgenic legume grains; they suggest that cyanic (e.g. red-brown) color may be engineered to mark genetically modified (GM) grains by the suppression of PA biosynthesis in the seed coat. Further, the reduction of PA amounts would be favourable for post-harvest processing of the GM grain, as PAs bind proteins and other metabolites, interfering with their extraction.

Our LC-MS/MS analysis of red-brown seed coat metabolites demonstrated that the major reduction in soluble PA content was accompanied by minor 
increases in the amounts of anthocyanin pigments (C3G and C3Ga) (Figure 5.2). However, red color in beans may not always be associated with increased anthocyanin content, as commercial dark red kidney beans (Phaseolus vulgaris L.) were identified to consist mainly of flavonol-glycosides and PAs with no anthocyanins identified by HPLC-DAD (Beninger and Hosfield, 1999). Other pigments identified from soybean seed coats that could provide a cyanic color include epiflavan-3-ol-anthocyanin conjugates, such as catechin-cyanidin-3-Oglucoside identified from the seed coat of black soybean cultivar Cheongja 3 (Lee et al., 2009), or 3-deoxyanthocyanidins, such as diosmetinidin identified from the seed coat of black soybean variety Clark (Kovinich et al., 2011b). However, these compounds were not identified as major pigments from the red-brown soybean seed coat by HPLC-DAD measuring absorbance at $520 \mathrm{~nm}$ (not shown) or as metabolites that have accumulated to different amounts compared to brown soybean by LC-MS/MS. Our LC-MS/MS results demonstrated that 3-Oglycosylated anthocyanins ( $\mathrm{C} 3 \mathrm{G}$ and $\mathrm{C} 3 \mathrm{Ga}$ ) were the main cyanic pigments present at significantly higher levels in the seed coat of red-brown soybean compared to the brown lines (Figure 5.2D).

Anthocyanin and PA biosynthesis are linked by the requirement for the common flavonoid intermediates, anthocyanidins; the PA enzyme anthocyanidin reductase (ANR) and the anthocyanin enzyme UDP-glycose:flavonoid-3-Oglycosyltransferase (UF3GT) both utilize cyanidin as a substrate (Figure 5.1). This suggests the possibility that the inhibition of PA biosynthesis due to a defect in ANR would render more cyanidin substrate available to UF3GT for 
anthocyanin biosynthesis, and could result in a red-brown seed coat phenotype. In Arabidopsis, mutations causing loss-of-function of the ANR gene (a.k.a. BANYULS) resulted in the loss of PAs in the seed coat and the temporary accumulation of red pigments (not formally identified as anthocyanins) during seed development (Devic et al., 1999). In soybean, our results demonstrated that defects in the ANR1 gene were accompanied by major reductions in PA content and minor elevations in the amounts of anthocyanins in the red-brown seed coat (Figure 5.2D). Although gene expression analysis demonstrated that ANR1 was highly expressed in the seed coat compared to ANR2 (Figure 5.4), it remains unclear whether the differences observed in gene expression or enzyme activity are sufficient to explain the phenotypic variation between brown and red-brown seed coats. Recently, a partial soybean sequence with $86 \%$ sequence identity to the $M$. truncatula ANR gene mapped to the genomic region coding the $O$ locus (Yang et al., 2010). The genes coding ANR1 (Glyma08g06630) and ANR2 (Glyma08g06640) were located in the same region of the Glyma1 soybean genome sequence, further supporting that either may encode the $O$ gene. However, further analysis, such as gene complementation or gene suppression will be required to substantiate that defects in the ANR1 gene are responsible for red-brown seed coat color and that $A N R 1$ may be the $O$ gene.

\subsection{Conclusion}

A better understanding of the biochemistry of red-brown seed coat color and the role of the soybean ANR1 gene in PA biosynthesis has been achieved in this 
work. We confirmed the in vitro catalytic function of two ANR genes isolated from the seed coat of brown soybean. Of the two genes, ANR1 was preferentially and highly expressed in the seed coat, suggesting a principal role in PA biosynthesis in this tissue. ANR1 exhibited reduced gene expression profiles in the seed coat of red-brown soybean relative to brown soybean and/or minor reductions in recombinant enzyme activity. Further analysis will be required to determine whether the differences observed in ANR1 gene expression or enzyme activity are sufficient to explain the phenotypic variation between red-brown and brown seed coats. Based on these results, we are presently attempting to suppress the ANR1 gene in brown soybean using RNA interference (RNAi) to determine whether such a mechanism will be sufficient to engineer a red-brown soybean grain color. We predict that the suppression of $A N R 1$ transcript profiles will result in the redirection of metabolic flux into the anthocyanin (and possibly other metabolite) pathways to produce a red-brown soybean grain color. If successful, the RNA interference construct could be linked to commercial transgenic traits to enable the visual detection of GM soybeans in the marketplace and abroad. 


\title{
CHAPTER 6
}

\section{Coloring genetically modified soybean grains with anthocyanins by the suppression of proanthocyanidin genes}

\author{
Adapted from: \\ Kovinich $\mathrm{N}^{1}$, Saleem $\mathrm{A}^{2}$, Rintoul $\mathrm{TL}^{3}$, Brown $\mathrm{DCW}^{5}$, Arnason $\mathrm{JT}^{4,5}$, and Miki $\mathrm{B}^{4,5}$. \\ Submitted. Aposomatic GM soybeans: Coloring genetically modified soybean grains with \\ anthocyanins by suppression of the proanthocyanidin genes ANR1 and ANR2. \\ Transgenic Research.

\footnotetext{
${ }^{1}$ Invented the approach of suppressing ANR genes to redirect metabolic flux into anthocyanin biosynthesis for coloring plant grains, designed and conducted research, analyzed data, and wrote the manuscript for publication

${ }^{2}$ Developed the multiple reaction monitoring (MRM) method for LC-MS/MS

${ }^{3}$ Transformed soybeans

${ }^{4}$ Invented the concept of 'coloring soybeans with anthocyanins' with V. De Luca (Brock University)

${ }^{5}$ Obtained funding for the research and revised the manuscript
}

\subsection{Introduction}

The increase in cultivation (Lemaux, 2008) and the diversification of commercial genetically modified (GM) crops for pharmaceutical (Karg and Kallio, 2009) and industrial (Basaran and Rodriguez-Cerezo, 2008) uses has brought added pressure for effective detection and monitoring of the adventitious presence of GM grains in non-GM materials (Demeke et al., 2006). Cases of adventitious presence are the basis for the opposition to $G M / n o n-G M$ coexistence (Ramessar et al., 2010) and typically result in severe economic penalties for exporters (Demeke et al., 2006; Karg and Kallio, 2009), international trade barriers (Smyth and McHughen, 2008), non-science-based regulations (Krueger and Le Buanec, 2008; Ramessar et al., 2010) and elevated public 
concern over the capability of regulatory bodies to effectively detect and monitor GM grains. Presently, the detection and quantification of the levels of adventitious presence are based on sophisticated tests (e.g. PCR, qPCR, and ELISA) (Demeke et al., 2006) that require a knowledge of molecular biology for proper interpretation of results and are not always reproducible (Morisset et al., 2009). Coloring transgenic plants, plant organs, and other transgenic materials intended for commercial markets to enable their visual identification could simplify the detection process and facilitate more effective monitoring and quantitation (Kovinich et al., 2011a).

Anthocyanins are flavonoid pigments found in plants that provide red-to-blue color to flowers, leaves, and grains. Proanthocyanidins (PAs) represent another class of flavonoid pigments that if present in plant grains provide a brown color when oxidized during desiccation. The biosynthesis of anthocyanins and PAs has been well-characterized over the past several decades, with numerous structural, regulatory, and transport genes identified (reviewed by Mol et al., 1998; WinkelShirley, 2001; Lepiniec et al., 2006; Zhao and Dixon, 2010). Although often studied separately, the biosynthesis of anthocyanin and PA pigments are intimately linked by their requirement for supply of flavonoid intermediate, cyanidin (Figure 6.1). The anthocyanin branchpoint enzyme UDPglycose:flavonoid-3-O-glycosyltransferase (UF3GT; EC 2.4.1.115) and the PA branchpoint enzyme anthocyanidin reductase (ANR; EC 1.3.1.77) both utilize the unstable flavonoid precursor, cyanidin, as a substrate (Figure 6.1). 


$$
\text { Chalcones }
$$

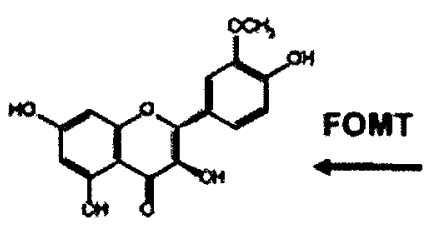
Isorhamnetin UGT78K2 UGT78K1

Isorhamnetin-3-0-glycoside (Flavonol glycosides)<smiles>C[C@@H]1CC[C@@H]([C@H]2O[C@@H]3C[C@H](O)C[C@H](O)[C@H]3[C@H](O)[C@H](O)[C@H]2O)C[C@H]1O</smiles>

Quercetin UGT78K2 UGT78K1 Quercetin-3-O-glycoside (Flavonol glycosides)

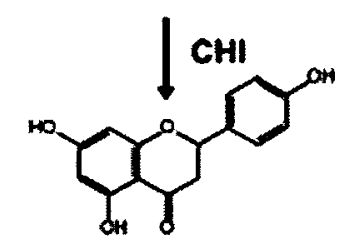

Naringenin

(Flavonones)

$\downarrow$ F3'H

I F $3 \mathrm{H}$

Dihydroflavonols

DFR2 DFR1

Leucoanthocyanidins

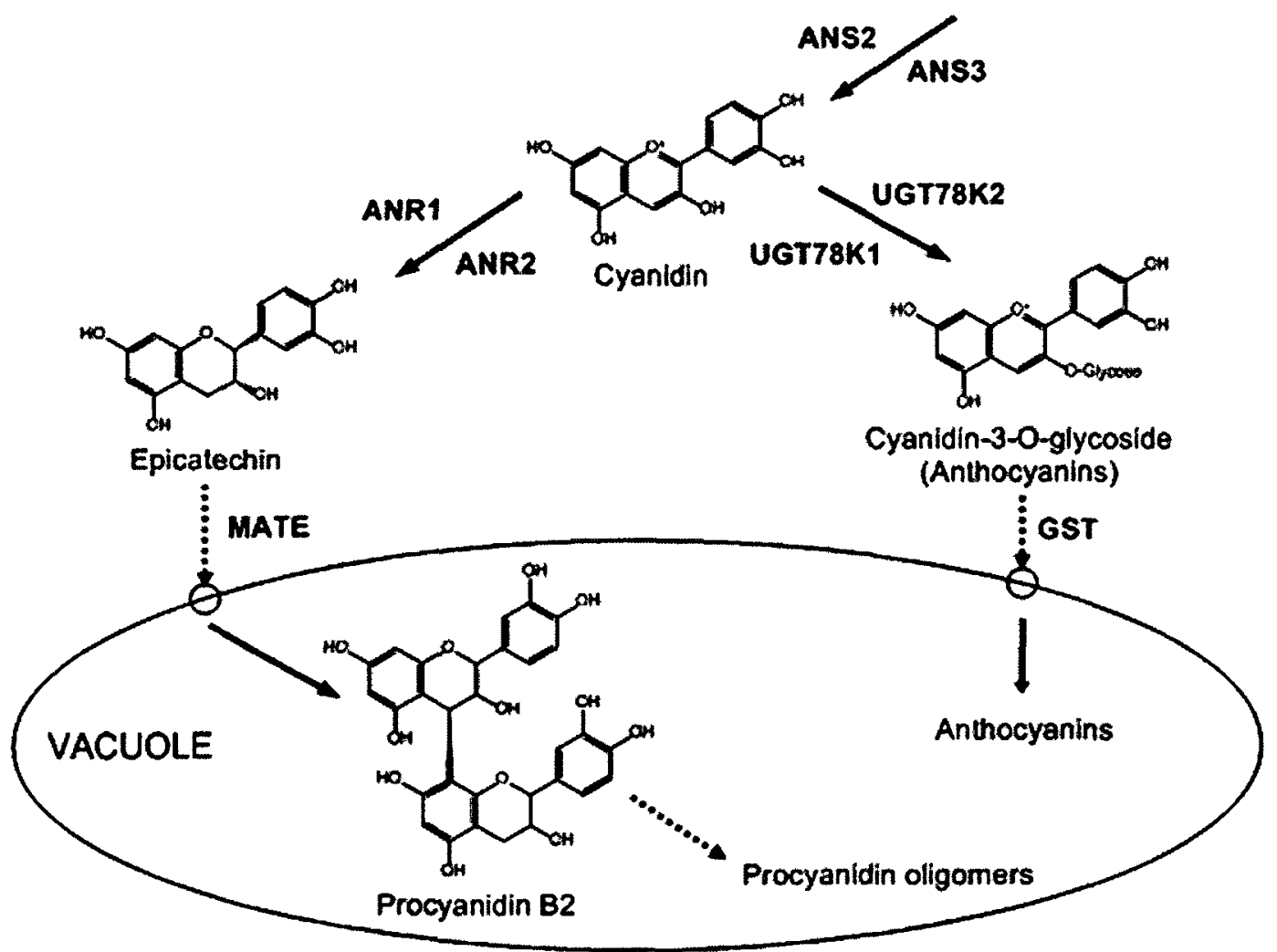

Figure 6.1. Simplified diagram of flavonoid biosynthesis in the seed coat of soybean variety Clark. See Kovinich et al. (2011b) for a more extensive diagram. 
Numerous studies have reported the successful manipulation of flower color as a result of engineering anthocyanin biosynthesis (reviewed by Holton, 1995; Tanaka et al., 1998; Nishihara and Nakatsuka, 2011). Further, flower color has been manipulated by redirecting metabolic flux into the anthocyanin pathway by suppression of the branchpoint gene for flavonol biosynthesis (FLS) (Holton et al., 1993; Tsuda et al., 2004; Nakatsuka et al., 2007). However, successful reports of engineering novel grain color are limited to studies that have overexpressed anthocyanin transcription factors in Maize (Shen and Petolino, 2006), Arabidopsis, and Tobacco (Geekiyanage, 2009). Engineering grain color by manipulating PA biosynthesis has not been reported, and PA gene knock-outs produce a transparent seed coat (testa) in the model plant Arabidopsis (Lepiniec et al., 2006) which suggests little potential for engineering grain color by manipulating PA genes.

We previously identified the anthocyanin branchpoint gene UGT78K1 encoding UDP-glycose:flavonoid-3-O-glycosyltransferase (UF3GT) from the black soybean seed coat and demonstrated its encoded activity in vitro, and by complementation of the Arabidopsis ugt78d2 knock-out mutation (Kovinich et al., 2010). Subsequently, a second UF3GT gene UGT78K2 was identified from the black seed coat by comparing metabolite and transcriptome data between isogenic black and brown soybean seed coats differing in alleles of the $R$ locus (Kovinich et al., 2011b). The PA branchpoint genes ANTHOCYANIDIN REDUCTASE1 (ANR1) and ANR2 were identified from the brown soybean seed coat using homology-based methods, and the activity of the encoded proteins for 
(-)-epicatechin synthesis were confirmed in vitro (Kovinich et al., 2011c). The same study demonstrated that reduced ANR1 gene expressions or activity to coincide with reduced amounts of soluble PAs, and elevated anthocyanin levels, in the red-brown seed coats of 2 naturally occurring and one EMS-mutagenesis mutant compared to 3 brown varieties (Kovinich et al., 2011c). Elevated anthocyanin levels in the red-brown seed coats did not coincide with elevated anthocyanin branchpoint gene expression, suggesting that a red-brown soybean grain color could be engineered simply by the suppression of the ANR1 gene.

In the present study, we found that a distinct red-brown soybean grain color could be engineered by the simultaneous suppression of the PA branchpoint genes ANR1 and ANR2. Gene suppression resulted in the increased accumulation of metabolites with expected and unexpected identities. Further genetic analysis identified the upregulation of flavonoid genes, suggesting positive feedback and feedforward mechanisms to be responsible for the increased accumulation of metabolites with unexpected identities in the soybean seed coat.

\subsection{Materials and Methods}

\subsubsection{Chemicals}

Cyanidin was purchased from Indofine (Somerville, NJ, USA), NADPH from Fisher Scientific (Ottawa, ON, CAN), (-)-epicatechin, procyanidin B2, cyanidin 3O-galactoside, and the 3-O-glucosides of cyanidin, quercetin and isorhamnetin were purchased from Extrasynthese (Lyon, FR). All chemicals were of the 
highest available purity. Solvents for metabolite extraction and LC-MS/MS were LC-MS grade (Fisher Scientific, Ottawa, ON, CAN).

\subsubsection{Plant materials and growth conditions}

Glycine max (L.) Merr. Variety Clark (PI547438) was obtained from the U.S. Department of Agriculture Soybean Germplasm Collection (Agricultural Research Service, University of Illinois at Champaign-Urbana). Seeds were surfacesterilized as described previously (Kovinich et al., 2011b), dried, and germinated in vermiculite in a Conviron $\mathrm{E} 15$ cabinet with a photoperiod of $16 \mathrm{~h}$ light $(590 \mu \mathrm{E}$ $\mathrm{m}^{2} \mathrm{~s}$ ) at $25^{\circ} \mathrm{C}$, and $8 \mathrm{~h}$ dark at $20^{\circ} \mathrm{C}$. After 12 days, seedlings were transplanted to soil and 30 days later the photoperiod was changed to $12 \mathrm{~h}$ light/12 $\mathrm{h}$ dark to encourage reproductive development. Pods were routinely harvested on ice and seed coats were dissected from pod and embryo material, immediately frozen in liquid nitrogen, lyophilized, and stored at $-80^{\circ} \mathrm{C}$ for future analysis.

\subsubsection{Nucleic acid isolation, vector construction, soybean transformation and} PCR genotyping

RNA was isolated from soybean seed coats using a previously described method (Wang and Vodkin, 1994), and further purified using the RNeasy Plant Mini Kit (QIAGEN, ON, Canada). The quantity and purity of RNA samples were determined by spectrophotometry using a NANODROP 2000 (Thermo Scientific, ON, Canada). RNA samples $(3 \mu \mathrm{g})$ were treated with DNase I (Amplification grade, Invitrogen, ON, Canada) at $37^{\circ} \mathrm{C}$ for 15 min prior to cDNA 
synthesis using SuperScript III Reverse Transcriptase (Invitrogen, ON, Canada) according to the manufacturer's instructions.

For assembly of the ANR-RNAi construct, $254 \mathrm{bp}$ of the 3'-CDS of ANR1 was amplified from seed coat cDNA isolated from the $25-50 \mathrm{mg}$ fresh seed weight (FSW) stage of seed development using primers ASFIASR (Appendix O). The amplicon was cloned into the pENTR/D-TOPO entry vector (Invitrogen), recombined into the pANDA35HK vector (Miki and Shimamoto, 2004) as described by the suppliers using the LR clonase enzyme mix (Invitrogen, ON, Canada), and the resultant ANR-RNAi construct was sequenced to confirm the integrity of the ANR1 hairpin. Soybean variety Clark was transformed with the ANR-RNAi construct by bombardment of embryonic suspension culture tissues as described by Finer and McMullen (1991). For genotyping, DNA was isolated and amplified using the REDExtract-N-Amp Plant PCR Kit (Sigma-Aldrich, Oakville, ON, CA) and the primers GUSF/GUSR (Appendix O).

\subsection{4. $q R T-P C R$}

qRT-PCR was performed on seed coat CDNA isolated from the $25-50 \mathrm{mg}$ FSW stage using $250 \mathrm{nM}$ of forward and reverse primers and the iQ SYBR Green Supermix (BioRad, ON, Canada) as described previously (Kovinich et al., 2011b). Parallel reactions were performed in the absence of Superscript III to test for contamination of genomic DNA. Gene expressions from each sample were normalized to the endogenous reference PEPC16. PCR cycling and qRT-PCR analysis of gene expressions and PCR efficiencies were analyzed using the 
Opticon Monitor 3 software (BioRad) as described previously (Kovinich et al., 2011b). To verify the specificity of the qRT-PCR reactions, melting curves were performed subsequent to each reaction in addition to fractionation of RT-PCR products on agarose gels. Primer sequences used in this study are listed in Appendix $\mathrm{O}$.

\subsubsection{Metabolite Analysis}

Lyophilized seed coats ( $400-500$ mg FSW) were ground to a fine powder in liquid nitrogen using an electric drill. Samples were aliquotted into $10-12 \mathrm{mg}$, and extracted with $\mathrm{MeOH}: \mathrm{H}_{2} \mathrm{O}: \mathrm{HCO}_{2} \mathrm{H}\left(80: 15: 5, \mathrm{v} / \mathrm{v} ; 20 \mu \mathrm{L} \mathrm{mg}{ }^{-1}\right)$ for $16 \mathrm{~h}$ on ice with shaking (250 rpm). Subsequent to centrifugation the supernatant was filtered through Teflon (0.2 $\mu \mathrm{m}$; Chromatographic Specialties). For compound identification, $5 \mu \mathrm{L}$ aliquots were analyzed by LC-MS/MS in scanning mode as described previously (Kovinich et al., 2011b). For compound quantification, $2 \mu \mathrm{L}$ aliquots were analyzed by LC-MS/MS in multiple reaction monitoring (MRM) mode in positive polarity at unit resolution. The system consisted of a 1200 series Agilent liquid chromatograph with high performance autosampler (model G1376B), binary pump (model G1312A), column thermostat (model G1316A), and PEEK $0.12 \mathrm{~mm}$ ID lines from column inlet onwards till tri-way splitter of a 3200 QTRAP with PEEK $0.17 \mathrm{~mm}$ ID $\times 60 \mathrm{~cm}$ lines on splitter outlet.

For MRM, two Q1 (parent) and Q3 (product) transitions were developed per authentic standard. The most sensitive transition was used for quantification and the second for authentication of identity. For quantitative optimization, authentic 
standards (10-20 $\mu \mathrm{g} \mathrm{mL}^{-1}$ dissolved in methanol) were infused into the turbo ion source using an electrospary probe at a flow rate of $10 \mu \mathrm{g} \mathrm{mL}^{-1}$. Flow Injection Analysis (FIA) was applied to optimize source conditions using the same LC conditions as for sample analysis; a linear gradient of $32-70 \% \mathrm{MeOH}$ in $5 \%$ $\mathrm{HCO}_{2} \mathrm{H}$ over $22 \mathrm{~min}$ at a flow rate of $1 \mathrm{~mL} \mathrm{~min}^{-1}$. The column (Luna C18 (2), 150x $4.6 \mathrm{~mm}, 5 \mu \mathrm{m}$ particle size, (Phenomenex Inc., CA, USA) was washed by ramping up the $\mathrm{MeOH} \%$ from $70-100 \%$ for 8 min. The initial conditions were brought back in $0.1 \mathrm{~min}$ and the column was equilibrated for 6 min before next injection. Samples were injected at a draw/inject speed of $200 \mu \mathrm{L} \mathrm{min}{ }^{-1}$. The injection needle was washed in a flush port for $3 \mathrm{sec}$ after every injection to minimize carry over. The column thermostat was $20^{\circ} \mathrm{C}$.

Detailed QTRAP settings for MRM analysis of the 6 investigated compounds are listed in Appendix P. Optimal signal-to-noise ratio was achieved with vertical and horizontal probe settings of $7 \mathrm{~mm}$ and $5 \mathrm{~mm}$, respectively. Quantitation of the 6 target compounds was performed using Analyst software 1.5.1. The integration of peaks was performed after applying a bunching factor/smoothing of 5 . The calibration curves were generated by diluting authentic standard mixes serially in methanol. Calibration curves were linear fit $\left(R^{2} \geq 0.998\right)$.

\subsection{Results}

6.3.1. Engineering red-brown soybean seed coats using the RNAi construct ANR-RNAi 
An RNA interference (RNAi) construct (ANR-RNAi) designed to silence ANTHOCYANIDIN REDUCTASE 1 (ANR1) was transferred into brown (irTO) soybean variety Clark using a biolistics protocol (Finer and McMullen, 1991). The ANR-RNAi construct consisted of 254-bp inverted repeats of the 3'-coding region of the ANR1 gene (Kovinich et al., 2011c) driven by the 35 S constitutive promoter and separated by a GUS linker sequence derived from the PANDA35HK vector (Miki and Shimamoto, 2004) (Figure 6.2A). Regenerated T0 plants were screened by PCR of the GUS linker sequence, and by the visual inspection of immature seeds dissected from pod material at various stages of development.

The three regenerated T0 lines ( $\mathrm{A} 1 \mathrm{~A} 1, \mathrm{~A} 1 \mathrm{~A} 2$, and $\mathrm{A} 1 \mathrm{~A} 3$ ) tested positive for the presence of the GUS linker sequence by PCR (GUS+), however only line A1A1 survived to reproductive maturity. Line A1A1 exhibited a novel red pigmentation in the hilum of immature seeds in the $<25-200 \mathrm{mg}$ fresh seed weight (FSW) stage of development (Figure 6.2B). This phenotype was not observed in the control (untransformed Clark) (Figure 6.2B), or in spontaneous or EMS-mutagenized red-brown varieties previously shown to have reduced ANR1 gene expression or enzyme activity (Kovinich et al., 2011c). The red pigment became visible over the entire seed coat surface by $200-300$ FSW stage of seed development (Figure 6.2C).

Selfing line A1A1 produced T1 progeny with 3 combinations of traits: 1) GUS+ progeny with red-brown grains, 2) GUS+ with brown grains identical to the 
A

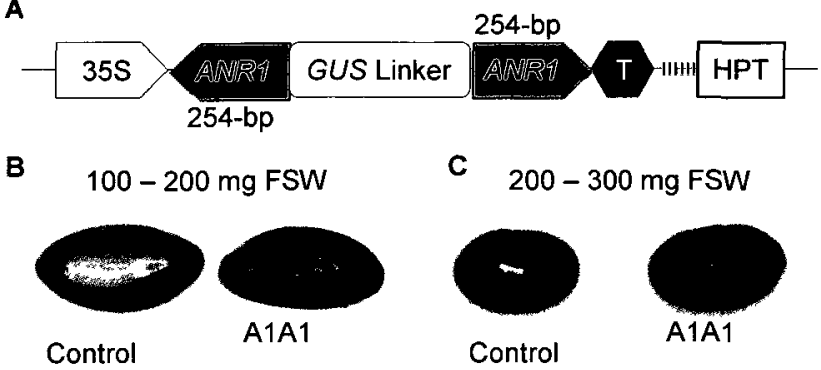

D

GUS

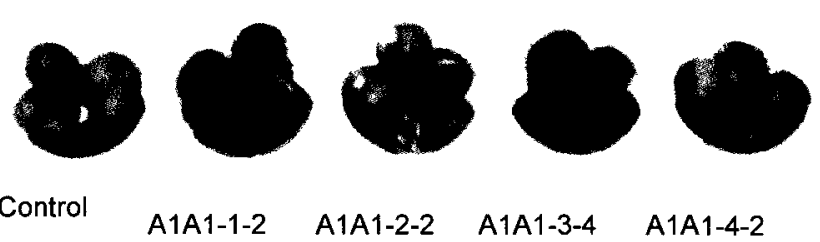

Figure 6.2. Structure of the ANR-RNAi DNA construct and phenotypes associated with its genomic integration in soybean. (A) Structure of the ANRRNAi DNA construct: 35 S promoter (35S); 3'-CDS of the G. max ANTHOCYANIDIN REDUCTASE1 (ANR1); GUS linker (GUS Linker); transcription terminator $(T)$; herbicide resistance gene hygromycin phosphotransferase (HPT). (B, C) Seed coat phenotypes at the $100-200 \mathrm{mg}$ fresh seed weight stage (FSW) and the $200-300 \mathrm{mg}$ FSW stage, respectively. Untransformed variety Clark (Control); T0 line A1A1 (A1A1). (D) PCR detection of GUS linker sequence from genomic DNA and dried grain phenotype of selected T1 lines. 
control, and 3) GUS- (tested negative for the presence of GUS by PCR) progeny with brown grains. The red-brown and brown grain color phenotypes observed in the T1 generation segregated stably with GUS linker sequences in the T2 and T3 generations, suggesting that multiple transgene insertions had been present in the parental T0 line. T2 lines representing all combinations of traits and containing single or multiple transgene insertions (as determined by inheritance of GUS and seed coat color in the T3 generation) were selected for in-depth analysis. Line A1A1-3-4 had a single transgene insertion and red-brown grain color, line A1A1-1-2 had multiple transgene insertions and a red-brown phenotype, line A1A1-2-2 was GUS- with a brown grain and was selected to represent the null segregating control, and line A1A1-4-2 had a multiple transgene insertions and a brown grain color. The grain phenotype and the detection of GUS linker sequence from genomic DNA by PCR are shown for each selected line in Figure 6.2D. GUS PCR products were sequenced to confirm their identity.

\subsubsection{High-level suppression of ANR1 and ANR2 genes by the ANR-RNAi} construct confers a red-brown grain phenotype while low-level suppression fails to modify seed coat color

To measure the level of gene suppression in transgenic lines carrying the ANR-RNAi construct, quantitative RT-PCR (qRT-PCR) of ANR genes was performed using seed coat cDNA isolated at the $25-50 \mathrm{mg} \mathrm{FSW}$ stage of seed development. The $25-50 \mathrm{mg}$ FSW stage was selected as the developmental time 
point for this analysis because ANR1 transcripts have been shown to be at maximum levels at this stage in the seed coat of brown (irT) soybean variety Clark (ir) (Kovinich et al., 2011b). To ensure the seeds of all lines were at the same stage of development, the days post anthesis, pod length, embryo morphology, and transcript levels of the developmental marker gene Gm-r10831191 were ensured to be equivalent among all materials prior to testing (Appendix Q). Comparisons of the ANR-RNAi sequence with other reductaseepimerase-dehydrogenase (RED) superfamily genes previously shown to be expressed in the seed coats of pigmented soybeans (Kovinich et al., 2011b, c) found ANR-RNAi to have high identity only with ANR1 (100\%) and ANR2 (86\%), and moderate identity (55-47\%) to other RED superfamily genes (Appendix R). For this reason we decided to include the RED superfamily genes with highest identity to ANR-RNAi (i.e. LAR1 and DFR1) in our qRT-PCR analysis to determine the specificity of gene silencing. Phosphenol pyruvate carboxylase 16 (PEPC16) was used as the endogenous reference for normalization of qRT-PCR measurements of target genes across seed coat samples as described previously (Sugimoto et al., 1992; Tuteja et al., 2004; Kovinich et al., 2011, 2011b).

Quantitative RT-PCR (qRT-PCR) revealed a 35.2 - 38.8 fold (97.1-97.4\%) reduction in the levels of ANR1 transcripts, and a $3.8-7.0$ fold $(73.5-85.7 \%)$ reduction in ANR2 transcripts in transgenic lines with a red-brown seed coat (A1A1-1-2 and A1A1-3-4), compared to the untransformed control or the segregating line (A1A1-2-2) that lacked the ANR-RNAi construct (Figure 6.3). By 


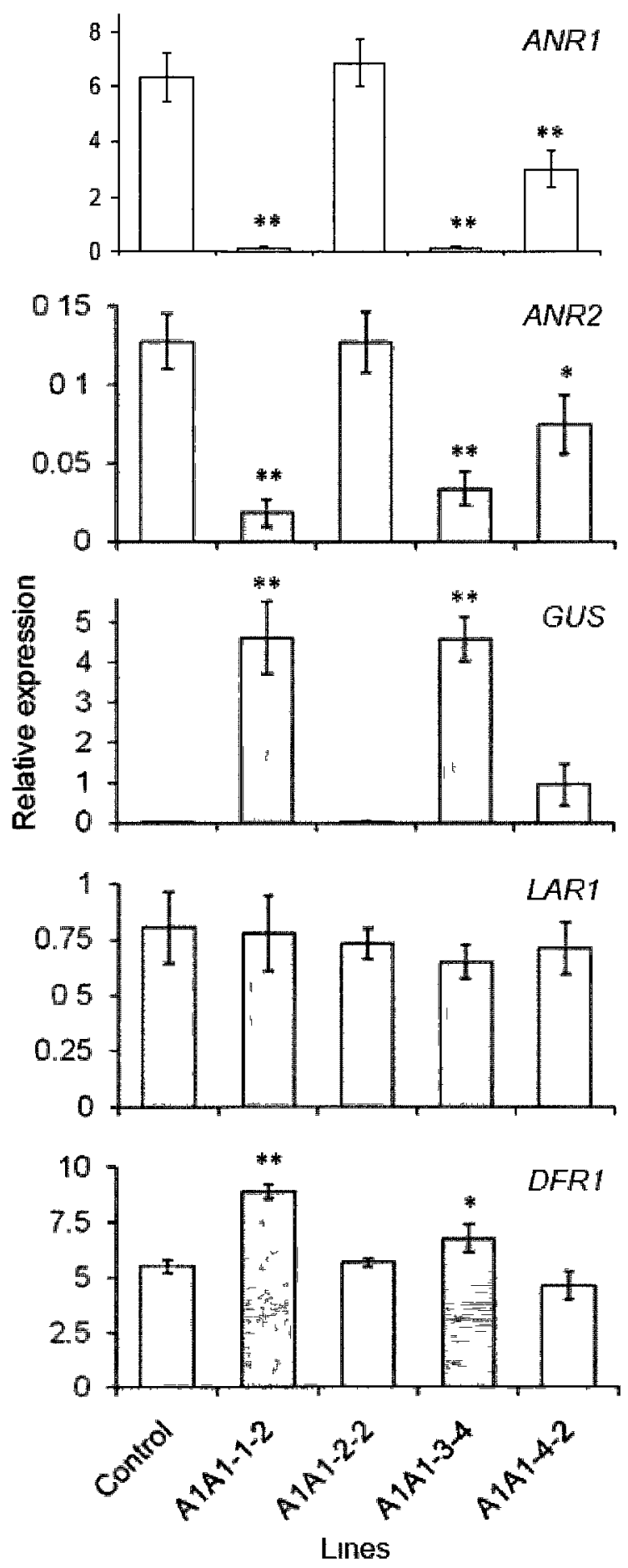

Figure 6.3. qRT-PCR measurement of transcript profiles from the seed coat of transgenic soybean carrying the ANR-RNAi construct. ANTHOCYANIDIN REDUCTASE1 (ANR1), ANR2, the ANR-RNAi construct GUS linker sequence (GUS), LEUCOANTHOCYANIDIN REDUCTASE1 (LAR1), and 
DIHYDROFLAVONOL 4-REDUCTASE1 (DFR1) profiles relative to the PEPC16 gene. Transgenic lines with red-brown seed coat color (grey bars), lines with control seed color (white bars). Values are mean and SD from three biological replicates. Student's $t$ test compared to the control significant at $\mathrm{P}<0.01\left(^{* *}\right), \mathrm{P}<$ $0.05\left(^{*}\right)$.

contrast, only a 2.1 and 1.7 fold reduction in the levels of ANR1 and ANR2 transcripts, respectively, was observed in line A1A1-4-2 (Figure 6.3), that tested GUS+ but contained the control phenotype (brown seed coat color) (Figure 6.2D). The magnitude of GUS linker sequence expression was inversely proportional to the level of ANR gene expression (Figure 6.3), supporting that ANR-RNAi expression was the causal agent of reduced ANR1 and ANR2 transcript levels in transgenic lines.

qRT-PCR demonstrated no significant decrease in the levels of $L A R 1$ or $D F R 1$ transcripts in transgenic lines relative to the control or the segregating GUS- line (Figure 6.3), suggesting that ANR-RNAi-mediated gene suppression was highly specific to $A N R 1$ and $A N R 2$ genes. Interestingly, $D F R 1$ expressions were upregulated $1.2-1.6$ fold in transgenic lines with a red-brown seed coat, suggesting that an endogenous positive feedback mechanism may have been stimulated in response to engineered ANR gene suppressions.

In summary, our results show high-level suppression of ANR1 and ANR2 genes in transgenic lines carrying the ANR-RNAi construct that have a red-brown seed coat color, and low levels of suppression in the GUS+ transgenic line that has the control (brown) seed coat phenotype. 
6.3.3. Red-brown transgenic grains undergoing high-level suppression of ANR1 and ANR2 genes have major reductions in the amount of PAs along with elevated levels of cyanic pigments in the seed coat

We previously demonstrated that proanthocyanidins (PAs) from brown or black seed coats of variety Clark are exclusively (-)-epicatechin based (Kovinich et al., 2011b). As ANTHOCYANIDIN REDUCTASE1 (ANR1) and ANR2 enzymes from G. max have been shown to convert cyanidin to (-)-epicatechin (Kovinich et al., 2011c), high-level suppression of ANR1 and ANR2 expressions by the ANRRNAi construct would be expected to cause significant reductions in the amounts of epicatechin-based PAs in the soybean seed coat.

Reaction of seed coat extracts with DMACA reagent revealed a $71.5-71.7 \%$ reduction (37.3-37.4 $\mathrm{mg} \mathrm{g}^{-1} \mathrm{LSC}$ ) in amounts of soluble PAs in the transgenic lines that had red-brown grain color and high-level ANR gene suppressions (A1A1-1-2 and A1A1-3-4), in comparison to the untransformed or segregating null controls (Figure 6.4A). By contrast, no significant change in soluble PA amounts relative to the controls was observed in the transgenic brown-colored 

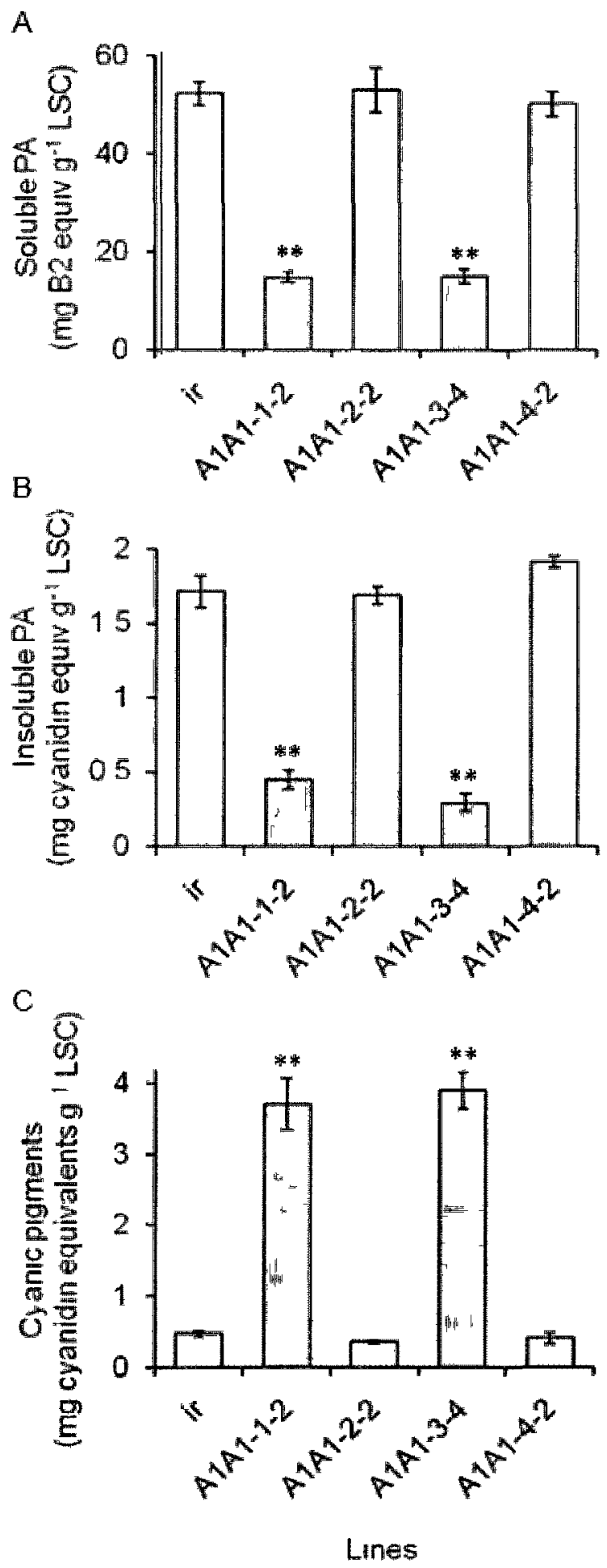

Figure 6.4. Total PA and cyanic pigment amounts from the seed coat of transgenic soybean carrying the ANR-RNAi construct expressed in units mg per gram lyophilized seed coat (LSC). (A) Soluble PAs measured by reaction of seed 
coat extracts with DMACA reagent, using the PA dimer procyanidin B2 (B2) as the quantitative standard. (B) Solvent insoluble PAs measured by acid catalyzed hydrolysis of previously extracted seed coat residues, quantitatively compared to the authentic standard cyanidin chloride. (C) Cyanic pigments (anthocyanins or anthocyanidins) measured by photospectrometry in comparison to cyanidin chloride. Transgenic lines with red-brown seed coat color (grey bars), lines with control seed color (white bars). Values are mean and SD from three biological replicates. Student's $t$ test compared to the control significant at $P<0.01\left(^{(*)}\right.$.

line (A1A1-4-3) (Figure 6.4A) that had only low levels of ANR gene suppression (Figure 6.2).

Measurement of solvent insoluble PAs by subjecting pre-extracted seed coat residues to acid-catalyzed hydrolysis revealed a $73.9-82.9 \%$ reduction (1.3-1.4 $\mathrm{mg} \mathrm{g}^{-1}$ LSC) in the amounts of solvent insoluble PAs in the red-brown transgenic lines (A1A1-1-2 and A1A1-3-4), and no significant change in amounts in the transgenic line that had the brown grain phenotype (A1A1-4-3), compared to the untransformed or segregating null controls (Figure 6.4B).

The red component of the red-brown grain color suggested elevated levels of cyanic pigment molecules (either anthocyanidins or their glycosides, the anthocyanins) in the red-brown transgenic seed coat. Measurement of the total amount of cyanic pigments from seed coat extracts was performed by photospectroscopy in comparison to the authentic standard cyanidin. Red-brown transgenic lines (A1A1-1-2 and A1A1-3-4) had a 7.9-8.3 fold increase (3.2-3.4 $\mathrm{mg} \mathrm{g}^{-1}$ LSC) in the total amounts of cyanic pigments compared to the control (Figure 6.4C). By contrast, the transgenic line with brown grain color (A1A1-4-3) 
that was phenotypically identical to the control exhibited no significant change in the amount of cyanic pigments compared to the control.

Overall, these results demonstrated that transgenic lines with engineered redbrown grain color by high-level suppression of ANR1 and ANR2 genes had major reductions in the amounts of soluble and solvent insoluble PAs, along with significant increases in the amounts of cyanic pigments (either anthocyanins or anthocyanidins).

\subsubsection{High-level suppression of soybean ANR1 and ANR2 genes redirects} metabolic flux from PA biosynthesis into the anthocyanin and flavonol-3-Oglucoside pathways

The enzymes anthocyanidin reductase (ANR) and UDP-glycose:flavonoid-3O-glycosyltransferase (UF3GT) utilize cyanidin as a substrate for proanthocyanidin (PA) and anthocyanin biosynthesis, respectively (Figure 6.1). As the ANR gene ANR1 and the UF3GT gene UGT78K2 were shown to be expressed at high and low levels in the brown soybean seed coat, respectively (Kovinich et al., 2011b), we hypothesized that suppression of ANR genes would redirect a moderate amount of metabolic flux from the PA pathway into the anthocyanin pathway, and would result in a phenotypic change from brown to a cyanic-colored soybean grain.

To identify metabolites that had accumulated differentially between transgenic and control seed coats, we analyzed seed coat extracts by liquid chromatography-tandem mass spectrometry (LC-MS/MS) in scanning mode. Six 
compounds were identified by LC-MS/MS to be present in different amounts in the transgenic red-brown seed coats compared to the untransformed control: (-)epicatechin, procyanidin B2, cyandin-3-O-glucoside (C3G), cyandin-3-Ogalactoside (C3Ga), quercetin-3-O-glucoside (Q3G), and isorhamnetin-3-Oglucoside (I3G) (Figure 6.5A).

To compare the amounts of each of these compounds between transgenic and control lines, LC-MS/MS was performed in multiple reaction monitoring (MRM) mode optimized for the detection of each of the six compounds. MRM revealed significant reductions in the amounts of (-)-epicatechin (38.8-41.5\%; 9.0-9.6 $\left.\mathrm{mg} \mathrm{g}^{-1} \mathrm{LSC}\right)$ and procyanidin B2 (52.1-62.2\%; 1.9-2.3 $\left.\mathrm{mg} \mathrm{g}^{-1} \mathrm{LSC}\right)$, along with increases in the amounts of the 3-O-glycosylated anthocyanins C3Ga (13.014.3 fold; $0.76-0.84 \mathrm{mg} \mathrm{g}^{-1}$ LSC) and C3G (2.5-2.7 fold; 0.27-0.32 $\mathrm{mg} \mathrm{g}^{-1} \mathrm{LSC}$ ), and the flavonol-3-O-glucosides Q3G (166.0-178.6 fold; $25.9-27.9 \mathrm{mg} \mathrm{g}^{-1}$ LSC) and I3G (8.0-9.6 fold; $\left.1.9-2.3 \mathrm{mg} \mathrm{g}^{-1} \mathrm{LSC}\right)$ from red-brown transgenic seed coat extracts (Figure 6.4B).

These results demonstrate that high-level suppression of soybean ANR1 and ANR2 genes redirects metabolic flux from PA biosynthesis into the anthocyanin pathway to produce a red-brown soybean seed coat. The major increase in flavonol-3-O-glucoside amounts in the transgenic seed coat was an unexpected result of engineering the suppression of ANR genes, as the branch into the 

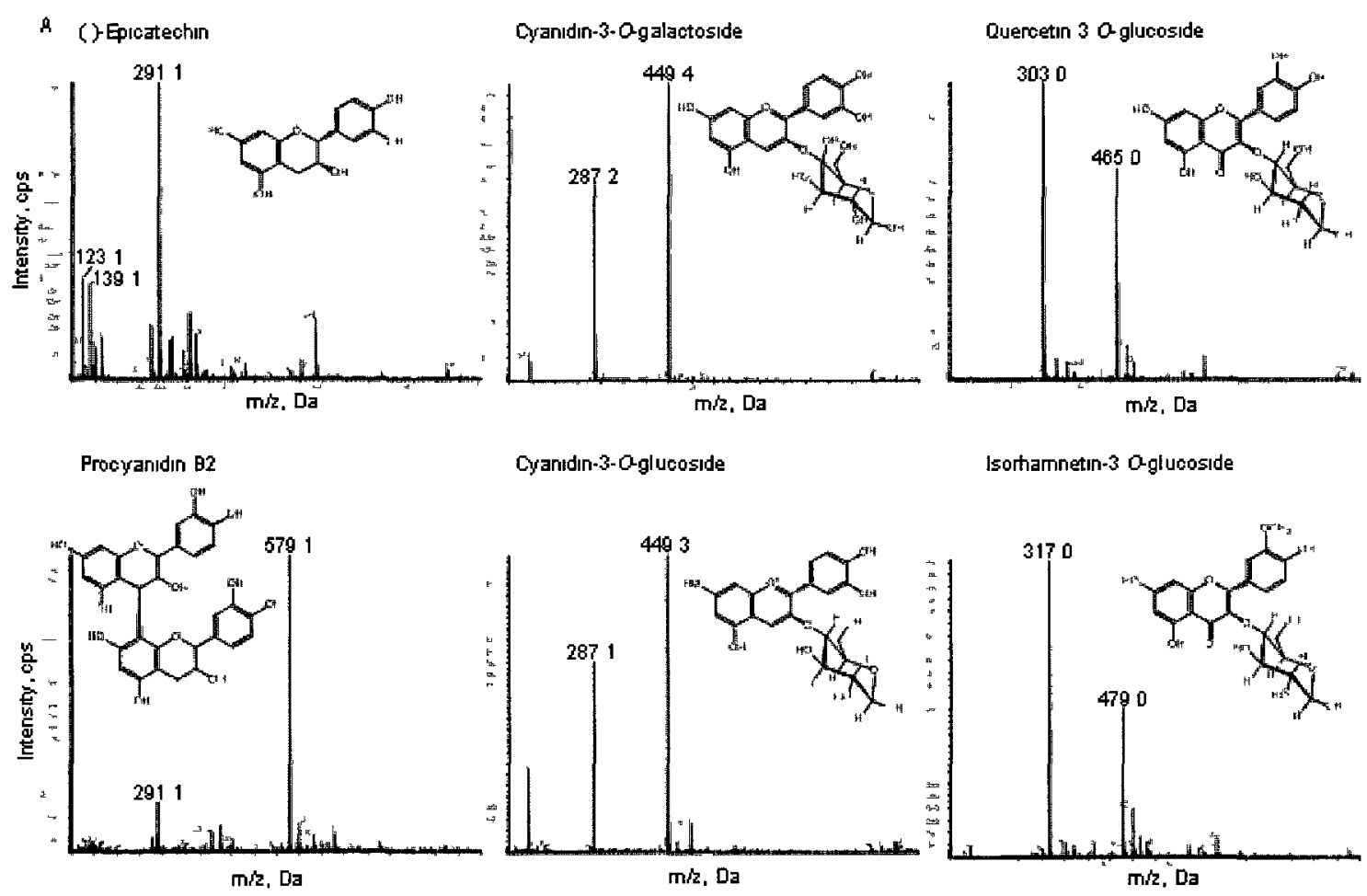

1sorhamnetın-3 Oglucoside
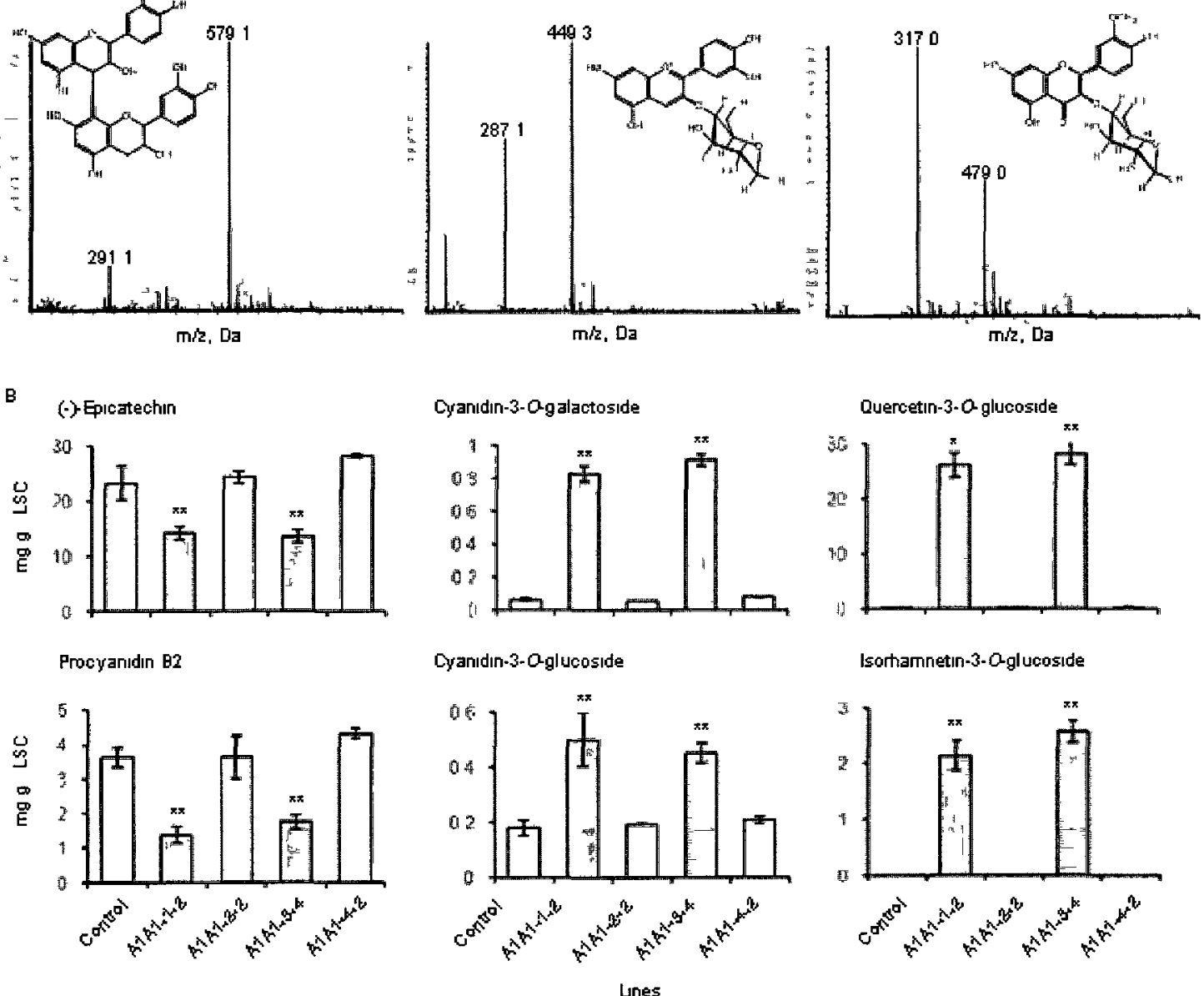

Figure 6.5. Identification and quantification of seed coat metabolites that accumulate to different amounts in transgenic lines with red-brown grain color compared to the untransformed control. (A) Mass spectra of (-)-epicatechin (Rt: $6.9 \mathrm{~min}$ ), cyanidin-3-O-galactoside (Rt: $\mathrm{min}$ ), quercetin-3-O-glucoside (Rt: 10.6 $\mathrm{min}$ ), procyanidin B2 (Rt: min), cyanidin-3-O-glucoside (Rt: $\mathrm{min}$ ), isorhamnetin-3O-glucoside (Rt: $12.4 \mathrm{~min}$ ). (B) Multiple reaction monitoring (MRM) of the 
compounds listed in (A) expressed in units $\mathrm{mg}$ per gram lyophilized seed coat (LSC). Transgenic lines with red-brown seed coat color (grey bars), lines with control seed color (white bars). Values are mean and SD from three biological replicates. Student's $t$ test compared to the control significant at $\mathrm{P}<0.01\left({ }^{* *}\right)$.

flavonol-3-O-glucoside pathway precedes the biochemical step catalyzed by ANR (Figure 6.1). These results suggest that metabolite differences resulting from the suppression of ANR genes may not be attributed solely to the increased accumulation of the ANR substrate, cyanidin.

\subsubsection{High-level suppression of soybean ANR1 and ANR2 genes causes the} upregulation of anthocyanin and flavonol-3-O-glucoside isogene transcripts

Metabolomes have a tendency to rebalance themselves in response to metabolic engineering events by mechanisms that are poorly understood. The overaccumulation of flavonol-3-O-glucosides in red-brown transgenic seed coats undergoing ANR gene suppression (Figure 6.5B) represented one such example. However, the unexpected upregulation of DFR1 transcripts (Figure 6.3) suggested transcriptional responses are involved in the mechanism responsible for re-routing metabolic flux. To gain further insight into this mechanism, we compared the expressions of several anthocyanin and flavonol-3-O-glucoside isogenes between transgenic red-brown seed coats and the control. As the suppression of ANR may be predicted to result in the accumulation of cyandin, (the substrate of ANR and the product of the anthocyanidin synthase (ANS) enzyme) (Figure 6.1), we investigated whether ANR suppression may cause 
alterations in the expressions of ANS genes (ANS2/ANS3). Furthermore, cyanidin is also the substrate for UDP-glucose:flavonoid-3-O-glucosyltransferase (UF3GT), which produces anthocyanins from anthocyanidins (Figure 6.1). For this reason, we also investigated whether ANR suppression may cause alterations in the expressions of UF3GT genes (UGT78K1 and UGT78K2).

qRT-PCR demonstrated 2.0-2.1 and 1.7-1.8 fold increases in the expressions of UGT78K2 and GST26, respectively, in red-brown transgenic seed coats undergoing ANR gene suppression compared to the control (Figure 6.6). By contrast, no significant differences in the expressions were observed for the UF3GT isogene UGT78K1, or the ANS genes ANS2/ANS3 (Figure 6.6).

Overall, the results from Figure 6.3 and Figure 6.6 demonstrate the upregulation of 3 flavonoid isogenes (DFR1, UGT78K2, and GST26) from transgenic red-brown soybean seed coats undergoing ANR gene suppressions. These results identify the upregulation of a network of gene transcripts as a component of positive feedback and feedforward mechanisms responsible for the redistribution of metabolic flux in response to ANR gene suppressions in the soybean seed coat.

\subsection{Discussion}

Previously we proposed that soybean grain color could be engineered by the suppression of anthocyanin and/or proanthocyanidin (PA) genes in the seed coat 

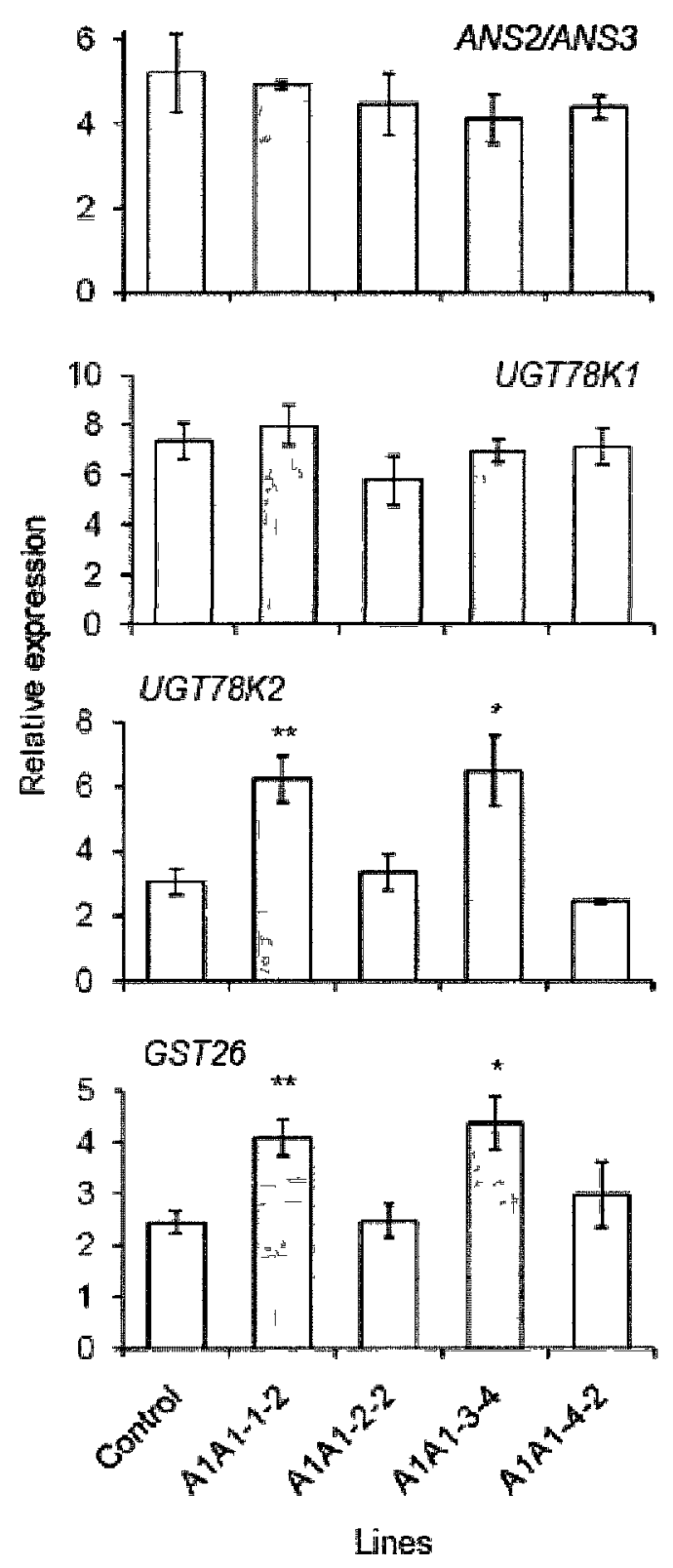

Figure 6.6. qRT-PCR measurement of transcript profiles identified positive feedforward/feedback induction of flavonoid gene expressions in the seed coat of transgenic soybean carrying the ANR-RNAi construct. ANTHOCYANIDIN SYNTHASE1/ANTHOCYANIDIN SYNTHASE2 (ANS1/ANS2), UDP. GLYCOSE:FLAVONOID-3-O-GLYCSYLTRANSFERASE isogene 1 (UGT78K1), UDP-GLYCOSE:FLAVONOID-3-O-GLYCSYLTRANSFERASE isogene 2 (UGT78K2), and putative anthocyanin GLUTATHIONE S-TRANSFERASE26 (GST26) profiles relative to the PEPC16 gene. Transgenic lines with red-brown seed coat color (grey bars), lines with control seed color (white bars). Values are 
mean and SD from three biological replicates. Student's $t$ test compared to the control significant at $\mathrm{P}<0.01\left(^{* *}\right), \mathrm{P}<0.05\left(^{*}\right)$.

to enable the visual detection of commercial transgenic grains (Kovinich et al., 2011a). Here we have reported the successful modification of soybean grain color (Figure 6.2D). This was achieved by the simultaneous and high-level suppression of the proanthocyanidin (PA) genes ANTHOCYANIDIN REDUCTASE1 (ANR1) and ANR2 (Figure 6.3). ANR1 and ANR2 are branchpoint genes into PA biosynthesis (Figure 6.1), and their high-level suppressions resulted in the reduction of total PAs (Figure 6.4), and the overaccumulation of specific anthocyanins (and unexpectedly flavonol-3-Oglucosides) in the soybean seed coat (Figure 6.5).

Since the first report of engineering novel flower color by the expression of the maize $D F R$ gene in petunia (Meyer et al., 1987), there have been numerous publications describing successful flower color manipulations in various plant species (reviewed by Holton, 1995; Tanaka et al., 1998; Nishihara and Nakatsuka, 2011). By contrast, successful reports of engineering novel grain color remain scarce, and may have been limited to the overexpression of anthocyanin transcription factors in Maize (Shen and Petolino, 2006), Arabidopsis, and Tobacco (Geekiyanage, 2009). The suppression of PA branchpoint genes in soybean to redirect metabolic flux into anthocyanin biosynthesis represented a novel approach for engineering pigmentation in plant grains. 
The re-channeling of flux between branches of the flavonoid metabolism has been observed previously. The dfr mutant had increased levels of flavonols in the Arabidopsis seed (Routaboul et al., 2006). The flavonol triple regulatory mutant pfg1 pfg 2 pfg 3 and the fls1-2 knock-out mutant each had increased accumulation of anthocyanins in the seedling (Stracke et al., 2007; Stracke et al., 2009). And the M. truncatula anthocyanin transporter mutant mate 2 had elevated levels of epicatechin-3'-O-glucoside in the flower (Zhao et al., 2011). Further, previous engineering experiments in soybean, Arabidopsis, and petunia have redirected metabolic flux from anthocyanin biosynthesis into the isoflavone pathway, from lignin biosynthesis into the flavonoid pathway, and from flavonol biosynthesis into the anthocyanin pathway, by suppressing anthocyanin, lignin, and flavonol branchpoint genes, respectively (Yu et al., 2003; Besseau et al., 2007; Nakatsuka et al., 2007). The overexpression of the ANR gene from Medicago truncatula in tobacco resulted in reduced anthocyanin pigmentation in the flower and elevated PA levels (Xie et al., 2003). These results demonstrated the potential for ANR to compete with the anthocyanin biosynthesis enzyme UDPglycose:flavonoid-3-O-glycosyltransferase (UF3GT) for the substrate anthocyanidin. Our results demonstrated the converse scenario; that suppression of ANR genes results in increased anthocyanin accumulations (Figure 6.4, Figure $6.5)$.

The Arabidopsis $A N R$ (a.k.a. BAN) knockout mutant displayed precocious accumulation of cyanic pigments in the seed coat during early seed development (Albert et al., 1997). The accumulations were only temporary, and resulted in a 
transparent testa (tt) phenotype with black pigmentation confined to the raphe of the dried grain (Albert et al., 1997). This contrasts the phenotype in soybean, where high-level suppression of ANR genes gives a red-brown grain (Figure 6.2D). There may exist underlying mechanistic and metabolite differences that could explain the differences in grain phenotypes between these species. In Arabidopsis, the UF3GT gene (UGT78D2) and the ANR gene are regulated reciprocally, with UGT78D2 expressed with anthocyanins in the seedling, and ANR expressed with PAs in the seed coat (Lee et al., 2005). By contrast, soybean UF3GT genes (UGT78K1 and UGT78K2) and ANR genes (ANR1 and $A N R 2$ ) are both expressed in the seed coat (Figure 6.3 and Figure 6.6). Thus, it is possible that the difference in phenotype between the soybean grain undergoing high-level ANR gene suppressions and the Arabidopsis ANR knockout grain, may be attributed to the presence and absence of UF3GT expressions, respectively, that stabilize anthocyanins in soybean allowing their accumulations to provide the red-brown grain phenotype. Consistent with this hypothesis, highlevel suppression of ANR genes in the soybean seed coat resulted in increased amounts of the enzymatic products of UF3GT (3-O-glycosylated anthocyanins) (Figure 6.5). It should be noted that the cyanic pigments that accumulated temporarily in the seed coat of the Arabidopsis ANR knockout mutant were identified by photospectrometric methods that could not distinguish between anthocyanins and the unstable anthocyanidins (Albert et al., 1997; Devic et al., 1999), and thus may not be 3-O-glycosylated anthocyanins. This hypothesis suggests that the expression of the UF3GT gene(s) may be an important 
consideration when designing strategies for the manipulation of grain pigmentation by the suppression of ANR genes in other species.

Feedback and feedforward mechanisms that operate during flavonoid biosynthesis remain poorly understood, and their elucidation could allow for more precise control over the outcomes of flavonoid engineering. Mechanisms of feedback and feedforward regulation of flavonoid and phenylpropanoid metabolism operate at the enzymatic level or the level of gene expression. Lphenylalanine ammonia lyase was shown to be inhibited by its own product, trans-cinnamic acid (Bolwell et al., 1986; Blount et al., 2000). The regulation of the bean CHS promoter by the first two intermediates of the phenylpropanoid pathway, trans-cinnamic acid and trans-p-coumaric acid, was observed in fungal elicitor treated alfalfa protoplasts (Loake et al., 1991). The Arabidopsis flavonol synthase (FLS) knockout mutant fls1-2 demonstrated the induction of $D F R$ and LDOX (a.k.a. ANS) genes (Stracke et al., 2009). Our results demonstrated the upregulation of $D F R 1, U G T 78 K 2$, and the putative anthocyanin glutathione Stransferase gene GST26 in response to ANR gene suppressions (Figure 6.3, Figure 6.6). These results suggest the upregulation of anthocyanin and flavonol gene transcripts to, at least in part, be responsible for the elevated levels of anthocyanins and flavonol-3-O-glucosides (Figure 6.5).

Interestingly, the transgenic line (A1A1-4-2) undergoing moderate levels of ANR gene suppression (Figure 6.3) did not exhibit a change in seed coat pigmentation relative to the untransformed or null segregating controls (Figure 6.2). This was consistent with the previous finding that a threshold of flavonoid 3'- 
hydroxylase gene down-regulation by virus-induced gene silencing (VIGS) had to be exceeded to cause a change in pubescence pigmentation in soybean (Nagamatsu et al., 2009). DFR1, UGT78K2 and GST26 were not upregulated in line A1A1-4-2 which suggested that the accumulation of upstream metabolites (possibly cyanidin), rather than suppression of ANR genes themselves, was responsible for the feedback/feedforward gene induction. The induction of DFR1, UGT78K2 and GST26 suggests the involvement of a regulatory factor in the feedback/feedforward induction mechanism, which is consistent with flavonoid genes being coordinately regulated (Hahlbrock et al., 1976; Pelletier et al., 1997; Hartmann et al., 2005). Further work is needed to determine how the overaccumulation of a flavonoid intermediate is perceived by the plant cell and the subsequent mechanisms that trigger the induction of flavonoid structural genes.

\subsection{Concluding Remarks}

The adventitious presence of genetically modified (GM) grains in non-GM shipments is the basis for opposition to GM/non-GM coexistence (Ramessar et al., 2010) and has resulted in elevated public concern over the capability of government bodies to effectively detect and monitor GM technologies. The use of fluorescent proteins (FPs) as transgene markers has been proposed to enable the optical detection of commercial genetically modified plant materials (Stewart, 2005, 2006), however FPs do not have a history of safe use for human consumption and their detection requires specialized equipment which may not 
allow for convenient detection by consumers, producers, processors, and importers. An alternative approach would be to color transgenic soybeans by altering their endogenous pigment metabolism to produce distinct colors of natural products that are currently already part of the food supply and that are generally recognized as safe (Kovinich et al., 2011a). Recently discovered branchpoint genes for soybean proanthocyanidin (PA) biosynthesis (namely, ANTHOCYANIDIN REDUCTASE1 (ANR1) and ANR2) and anthocyanin biosynthesis (UGT78K1 and UGT78K2) have been identified in addition to comparative biochemical analyses of soybean lines differing in their expressions and seed coat color (Kovinich et al., 2010, 2011b, 2011c). This has enabled the design of a rationale approaches for engineering soybean grain color. Here we have demonstrated one such approach; the production of a red-brown grain by the suppression of PA branchpoint genes ANR1 and ANR2 to redirect metabolism into anthocyanin pigment biosynthesis. Coloring transgenic soybean grains intended for commercial markets to enable their visual detection may provide increased safety features to consumers and increased freedom to producers (Kovinich et al., 2011a). The use of recombinant DNA technology, rather than traditional breeding or EMS-mutagenesis, offers the advantage of physically linking the DNA element for "grain-coloring" to the transgene(s) that encode the desired commercial trait. Reducing the amount of PAs in transgenic soybean grains by the suppression of PA biosynthesis represents an advantageous approach to engineering a visible marker for industrial, pharmaceutical, or other commercial grains designed to produce proteins, as PA 
pigments are known to bind proteins and complicate their extraction during processing. As the prevalence and diversity of transgenic materials increases, the use of different color markers to distinguish materials engineered for pharmaceutical, industrial, and other non-food/feed commercial uses may become increasingly warranted. 


\section{CHAPTER 7}

\section{General discussion}

\subsection{How does this study fit in existing societal, methodological and theoretical debates?}

\subsubsection{The societal debate}

Numerous incidents have occurred where GM materials have contaminated non-GM shipments. In October 2009, the European Union halted the importation of all North American soybeans due to contamination by tiny amounts of unapproved GM maize types (Michael Hogan, Reuters - HAMBURG, Nov 2, 2009). The European Union stopped importation of flax (Linum usitatissimum L.) seed from Canada due to illegal contamination by GMOs, threatening a lucrative \$320-million annual market (Martin Mittelstaedt, Globe and mail, October 2009). The biotechnology giant ProdiGene Inc was forced to purchase 500,000 bushels of potentially contaminated soybeans at a cost of $\$ 2.8$ million and pay a $\$ 250,000$ fine due to their failure to contain corn producing a pig vaccine (Karg and Kallio 2009). These and other unfortunate incidents have resulted in elevated public concern over the capability of government bodies to effectively detect GM grains and could potentially have been prevented.

Presently, the detection and quantification of the levels of adventitious presence are based on sophisticated tests (e.g. PCR, qPCR, and ELISA) (Demeke et al., 2006) that require a knowledge of molecular biology for proper interpretation of results and are not always reproducible (Morisset et al., 2009). 
The use of fluorescent proteins (FPs) as transgene markers has been proposed to enable the optical detection of commercial genetically modified plant materials (Stewart, 2005, 2006). Although green fluorescent protein (GFP) has been shown to be safe for consumption in tests using laboratory rats (Richards et al., 2003), FPs do not have a history of safe use for human consumption nor are they present in commercial GM crops presently on the market. Further, the detection of FPs requires specialized equipment that may not allow for convenient detection by importers, processors, producers, and consumers.

The main objective of our study, to engineer colored soybeans by suppression of anthocyanin and/or PA genes, was intended to provide a safe, simple, and effective alternative technology to enable the detection of GM grains. Several lines of GM carnation (Dianthus caryophyllus) with modified flower color are commercially available (www.cera-gmc.org). This exemplifies consumer acceptance of GM plant materials colored by genetic engineering. However, it remains to be determined whether consumers would similarly accept a safe edible plant material colored by genetic engineering.

As the soybean market becomes more fragmented through the introduction of new attribute-enhanced (a.k.a. identity preserved) varieties, there will be a need to expand the current storage and handling infrastructure in Ontario to accommodate more soybean varieties than are currently handled (Soy $20 / 20$, 2010). The use of grain coloring technologies to enable the efficient detection of adventitious presence may be economically advantageous for Canada. Such technologies would enable more effective identification and monitoring of GM 
grains intended for specific uses, and would reduce the incidence of adventitious presence. This in turn, would likely increase consumer confidence and could ease the GM/non-GM coexistence debate.

\subsubsection{The methodological debate}

Numerous studies have reported the successful manipulation of flower color as a result of engineering anthocyanin biosynthesis (reviewed by Holton, 1995; Tanaka et al., 1998; Nishihara and Nakatsuka, 2011). However, successful engineering of target colors is generally uncommon, as exemplified by more than one decade of attempts to engineer a true blue rose (Holton and Tanaka, 1994; Katsumoto et al., 2007). Reports of successfully engineering novel grain color have remained scarce (Shen and Petolino, 2006; Geekiyanage, 2009). For these reasons, our objective to engineer red-brown or red soybean grain colors by the suppression of anthocyanin or PA genes, respectively, was continuously met with skepticism from experts of metabolic engineering. This positioned our work in the center of a methodological debate as to whether grain color could be engineered simply by gene suppressions.

Prior to initiating our work we had two hypotheses: 1) The suppression of the UF3GT gene(s) in the seed coat of black soybean would result in reduced anthocyanin accumulations and produce a red-brown grain color, and 2) the suppression of the ANR gene(s) in the seed coat of brown soybean would redirect metabolic flux into the anthocyanin pathway and produce a red grain color. We were unable to test the first hypothesis because no viable 
transformants were obtained from transformation of soybean with a constitutively-expressed construct (i.e. pANDA35HK encoding the $35 \mathrm{~S}$ promoter) designed to silence the UF3GT gene UGT78K1. This may be attributed to the low efficiency of transformation of soybean variety Clark, or it may suggest that UF3GT may be essential for soybean viability. However, we were able to test hypothesis 2) and were successful in demonstating that GM soybean grain color could be modified by the suppression of ANR genes. As PAs are believed to be responsible for the brown color of seeds (Lepiniec et al., 2006), we hypothesized that suppression of PA genes in a brown seed coat would remove the brown pigmentation result in an anthocyanin-based red grain color. However, the result of suppression of ANR genes was actually a red-brown grain color, suggesting that flavonols may also oxidize to brown in the dried grain.

Suppression of PA genes represents a novel approach for engineering pigmentation in plant tissues that had not been reported previously. The possibility exists that suppression of PA genes could modify flower color in some species. The possibility also exists that this technology could be adapted for coloring the GM grains of other important crop species. For example, the pigmented husks of wheat (Triticum spp.) and canola (Brassica napus L.) accumulate PAs (McCallum and Walker, 1990; Naczk et al., 1994) and ESTs representing ANR (a.k.a. BANYULS) gene(s) have been recently identified from the seed coat of flax (Linum usitatissimum) (Venglat et al., 2011).

PA deposition in the seed coat has a role in defense against predators and pathogens (Winkel-Shirley 1998). However, commercial yellow soybeans lack 
PAs, so red-brown seed coats with reduced PA amounts may not be expected to have reduced levels of pathogen defense compared to the yellow varieties. Also, no differences in plant or seed development were observed to result from the constitutive expression of the ANR-RNAi construct by the $35 \mathrm{~S}$ promoter. This may be due to the fact that PAs accumulate in the seed coat of soybean and are not essential for development, as exemplified by their absence in yellow soybean genotypes.

Soybean is a self-pollinating plant species. However, in the rare event that red-brown GM soybean plants were to outcross to commercial yellow varieties, the red-brown trait would not be visible due to the natural RNA interference (RNAi) of the CHS gene by the / locus. For this reason, transformation of elite commercial yellow soybean varieties with a CHS gene that evades RNA interference by the / locus in addition to the ANR-RNAi element would likely be a superior approach for coloring commercial GM soybean grains.

Presently, there are no commercial GM crop plants that utilize RNAi as the transgenic trait. This is likely due to ongoing patent issues rather than safety reasons. Rice contains a very large number of short double-stranded RNA (dsRNA), many of which have identical sequences to segments of various human genes (Ivashuta et al., 2009). This means that humans have a long history of safe exposure to dsRNA's that can mediate RNAi in foods, such as plant-derived RNAs that are identical to segments of our own genes.

The suppression of ANR1 and ANR2 in the GM soybean seed coat caused a reduction of PA amounts to levels that are within the range found in brown and 
yellow soybean seed coats. However, the suppression of PA genes was accompanied by the unexpected overaccumulation of high amounts (25.9-27.9 $\mathrm{mg} \mathrm{g}^{-1}$ LSC) of quercetin-3-O-glucoside (Q3G). Onion (Allium cepa) represents a food that has relatively high amounts of Q3G (upto $1 \mathrm{mg} \mathrm{g}^{-1}$ fresh weight) (Hertog et al., 1992; Crozier et al. 1997), however our engineered seed coats had roughly 26 times this amount. This data suggests that Q3G levels in the engineered seed coats may be out of the range that is found in foods, and should undergo additional safety testing before the GM grain can be considered safe for consumption. As quercetin has been shown to have a range of activities (e.g. anti-carcinogenic, anti-hypertensive, anti-diabetic, and antioxidant) in vivo, this data also suggests that our engineered red-brown seed coats could be a valuable commercial source for the quercetin-3-O-glucoside.

\subsubsection{The theoretical debate}

Feedback and feedforward mechanisms have remained largely ignored in models describing the control of flavonoid biosynthesis. The unexpected feedback and feedforward mechanisms observed in our work support the view that such mechanisms must be elucidated to enable more predictive outcomes of metabolic engineering. These unexpected results illustrate the importance of basic research prior to product development.

\subsection{Original contributions}


Our comparative analyses of metabolites from black, brown, and red-brown soybean seed coats by LC-MS/MS provided a better understanding of the relationship between seed coat composition and grain color.

Previous to our work, genes for only 4 flavonoid enzymes (CHS, F3H, F3'H, and $\mathrm{F}^{\prime} \mathrm{5}^{\prime} \mathrm{H}$ ) were identified to be involved in pigment biosynthesis in the soybean seed coat (Todd and Vodkin, 1996; Toda et al., 2002; Zabala and Vodkin, 2003; Zabala and Vodkin, 2005; Zabala and Vodkin, 2007; Kovinich et al., 2010). We identified more than 20 isogenes for 14 proteins to have putative roles in flavonoid biosynthesis in black (iRT) or brown (irT) seed coats using a combined analysis of microarray and metabolite data. Further, the functions of 5 genes (UGT78K1, UGT78K2, OMT5, ANR1 and ANR2) were characterized in vitro, by assay of recombinant enzyme activities, and in vivo by complementation (UGT78K1) and gene suppression (ANR1 and ANR2). Our combined analyses of metabolite and gene expression data have provided a better understanding of soybean seed coat metabolism and grain color.

Our work demonstrated the concept that soybean seed coat color could be modified by metabolic engineering to enable the visible identification of the genetically modified (GM) grain.

The suppression of proanthocyanidin (PA) branchpoint genes in soybean to redirect metabolic flux into anthocyanin biosynthesis represented a novel approach for engineering pigmentation in plants.

We identified positive feedback and feedforward mechanisms in the flavonoid biosynthetic pathway in the soybean seed coat. We identified that the induction 
of flavonoid genes in response to ANR gene suppressions was, at least in part, responsible for the redirection of metabolic flux into anthocyanin biosynthesis.

\subsection{Future research}

The successful engineering of a red-brown soybean grain color raises the question of whether these results may be extended to produce additional seed coat colors for GM soybeans that have alternative uses. For example, could a blue grain color be produced by introducing into the ANR-RNAi construct an element that would silence the flavonoid $3^{\prime}$-hydroxylase $\left(F 3^{\prime} H\right)$ gene? This may be expected to redirect metabolic flux into the delphinidin (blue) branch of the anthocyanin pathway. Alternatively, could a red grain be produced by expressing a synthetic or orthologous chalcone synthase (CHS) gene with the ANR-RNAi construct in a yellow grain? The synthetic or orthologous $\mathrm{CHS}$ gene could be designed to evade the post-transcriptional silencing response of the / locus, and its expression may be expected to drive metabolic flux into the flavonoid pigmentation pathways.

Feedback and feedforward mechanisms that operate during flavonoid biosynthesis remain poorly understood, and their elucidation could allow for more precise control over the outcomes of flavonoid engineering. Our results suggested that the overaccumulation of a flavonoid intermediate (possibly cyanidin), rather than suppression of ANR genes themselves, was responsible for triggering the feedback/feedforward gene induction. The identification of the exact metabolite (or metabolites) that trigger the induction of flavonoid structural 
genes, and the elucidation of the mechanisms that lead to gene induction, would represent significant advancements in our understanding of flavonoid metabolism, and should be considered as important objectives for future research.

\subsection{Implications and concluding remarks}

Our work demonstrated that the seed coat color could be engineered to enable the visible identification of the GM soybean grain. We identified numerous pigmentation genes that could be manipulated to provide different colors to GM soy grains that are intended for different uses. This type of technology would simplify GM grain detection and may lead to fewer incidences of adventitious presence, which would undoubtedly have favourable economic, regulatory, and social implications.

Our work demonstrated that the suppression of PA biosynthesis caused the overaccumulation of anthocyanins and unexpectedly flavonol glycosides. We identified positive feedback and feedforward mechanisms as a component of the underlying mechanism. Harnessing feedback and feedforward controls in addition to other levels of metabolism regulation will likely expand the boundaries of what can be achieved by metabolic engineering. 


\section{REFERENCES}

Albert S, Delseny M, Devic M (1997) BANYULS, a novel negative regulator of flavonoid biosynthesis in the Arabidopsis seed coat. The Plant journal: for cell and molecular biology 11: 289-299

Alfenito MR, Souer E, Goodman CD, Buell R, Mol J, Koes R, Walbot V (1998) Functional complementation of anthocyanin sequestration in the vacuole by widely divergent glutathione S-transferases. Plant Cell 10: 1135-1149

Almeida JR, D'Amico E, Preuss A, Carbone F, de Vos CH, Deiml B, Mourgues F, Perrotta G, Fischer TC, Bovy AG, Martens S, Rosati C (2007) Characterization of major enzymes and genes involved in flavonoid and proanthocyanidin biosynthesis during fruit development in strawberry (Fragaria xananassa). Arch Biochem Biophys 465: 61-71

Alonso JM, Stepanova AN, Leisse TJ, Kim CJ, Chen H, Shinn P, Stevenson DK, Zimmerman J, Barajas P, Cheuk R, Gadrinab C, Heller C, Jeske A, Koesema E, Meyers CC, Parker H, Prednis L, Ansari Y, Choy N, Deen H, Geralt M, Hazari N, Hom E, Karnes M, Mulholland C, Ndubaku R, Schmidt I, Guzman P, Aguilar-Henonin L, Schmid M, Weigel D, Carter DE, Marchand T, Risseeuw E, Brogden D, Zeko A, Crosby WL, Berry CC, Ecker JR (2003) Genome-wide insertional mutagenesis of Arabidopsis thaliana. Science 301: 653-657

Basaran P, Rodriguez-Cerezo E (2008) Plant molecular farming: opportunities and challenges. Critical reviews in biotechnology 28: 153-172

Beninger CW, Hosfield GL (1999) Flavonol glycosides from Montcalm dark red kidney bean: implications for the genetics of seed coat color in Phaseolus vulgaris L. J. Agric. Food Chem. 47: 4079-4082

Benjamini Y, Hochberg Y (1995) Controlling the false discovery rate: a practical and powerful approach to multiple testing. J R Stat Soc Ser B 57: 289-300

Bernard R, Weiss M (1973) Qualitative genetics. In Soybeans: Improvement, Production, and Uses, 1st ed. Madison, WI: American Society of Agronomy pp 117-154

Besseau S, Hoffmann L, Geoffroy P, Lapierre C, Pollet B, Legrand M (2007) Flavonoid accumulation in Arabidopsis repressed in lignin synthesis affects auxin transport and plant growth. The Plant cell 19: 148-162

Blount JW, Korth KL, Masoud SA, Rasmussen S, Lamb C, Dixon RA (2000) Altering expression of cinnamic acid 4-hydroxylase in transgenic plants provides evidence for a feedback loop at the entry point into the phenylpropanoid pathway. Plant physiology 122: 107-116

Bolwell G, Cramer C, Lamb C, Schuch W, Dixon R (1986) L-Phenylalanine ammonia-lyase from Phaseolus vulgaris: modulation of the levels of active enzyme by trans-cinnamic acid. Planta 149: 97-107

Boveris AD, Galatro A, Sambrotta L, Ricco R, Gurni AA, Puntarulo S (2001) Antioxidant capacity of a 3-deoxyanthocyanidin from soybean.

Phytochemistry 58: $1097-1105$ 
Brazma A, Hingamp P, Quackenbush J, Sherlock G, Spellman P, Stoeckert C, Aach J, Ansorge W, Ball CA, Causton HC, Gaasterland T, Glenisson P, Holstege FC, Kim IF, Markowitz V, Matese JC, Parkinson H, Robinson A, Sarkans U, Schulze-Kremer S, Stewart J, Taylor R, Vilo J, Vingron M (2001) Minimum information about a microarray experiment (MIAME)-toward standards for microarray data. Nature genetics 29: 365-371

Butelli E, Titta L, Giorgio M, Mock HP, Matros A, Peterek S, Schijlen EG, Hall RD, Bovy AG, Luo J, Martin C (2008) Enrichment of tomato fruit with health-promoting anthocyanins by expression of select transcription factors. Nature biotechnology 26: 1301-1308

Buzzell R, Buttery B, MacTavish D (1987) Biochemical genetics of black pigmentation of soybean seed The Journal of Heredity 78: 53-54

Choung M, Baek I-Y, Kang S-T, Han W-Y, Shin D-C, Moon H-P, Kang K-H (2001) Isolation and Determination of Anthocyanins in Seed Coats of Black Soybean (Glycine max (L.) Merr.). J. Agric. Food Chem. 49,: 58485851

Clough SJ, Bent AF (1998) Floral dip: a simplified method for Agrobacteriummediated transformation of Arabidopsis thaliana. Plant $\mathrm{J}$ 16: 735-743

Crozier A, McDonald MS, Lean MEJ, Black C (1997) Quantitative analysis of the flavonoid content of tomatoes, onions, lettuce and celery. J. Agric. Food Chem. 45: 590-595.

Demeke T, Perry D, Scowcroft W (2006) Adventitious presence of GMOs: Scientific overview for Canadian grains. Can. J. Plant Sci. 86: 1-23

Devic M, Guilleminot J, Debeaujon I, Bechtold N, Bensaude E, Koornneef M, Pelletier G, Delseny M (1999) The BANYULS gene encodes a DFR-like protein and is a marker of early seed coat development. Plant $\mathrm{J}$ 19: 387398

Dhaubhadel S, Gijzen M, Moy P, Farhangkhoee M (2007) Transcriptome analysis reveals a critical role of CHS7 and CHS8 genes for isoflavonoid synthesis in soybean seeds. Plant physiology 143: 326-338

Dixon RA (2005) Engineering of plant natural product pathways. Current opinion in plant biology 8: 329-336

Downey MO, Harvey JS, Robinson SP (2003) Analysis of tannins in seeds and skins of Shiraz grapes throughout berry development. Aust J Grape Wine Res 9: 15-27

Doyle A (2008) EMBARGOED-World fails to monitor biotech trade-UN study. In $R$ Williams, ed. Thomson Reuters

Fedoroff NV, Furtek DB, Nelson OE (1984) Cloning of the bronze locus in maize by a simple and generalizable procedure using the transposable controlling element Activator (Ac). Proc Natl Acad Sci U S A 81: 38253829

Finer JJ, McMullen MD (1991) Transformation of soybean via particle bombardment of embryogenic suspension culture tissue. In Vitro Cell. Dev. Biol._Plant 27: 175-182 
Ford CM, Boss PK, Hoj PB (1998) Cloning and characterization of Vitis vinifera UDP-glucose:flavonoid 3-O-glucosyltransferase, a homologue of the enzyme encoded by the maize Bronze- 1 locus that may primarily serve to glucosylate anthocyanidins in vivo. J Biol Chem 273: 9224-9233

Forkmann G, Heller W (1999) Biosynthesis of flavonoids. In U Sankawa, ed, Comprehensive Natural Products Chemistry: Polyketides and Other Secondary Metabolites Including Fatty Acids and Their Derivatives, Vol I. Elsevier Science Publishers, Amsterdam, pp 713-748

Geekiyanage S (2009) Potential of VImybAl-2 as a candidate marker for visual identification of transgenic plants: induced anthocyanin production in Arabidopsis and tobacco. Trop Ag Res \& Ext 12: 35-41

Gentleman RC, Carey VJ, Bates DM, Bolstad B, Dettling M, Dudoit S, Ellis B, Gautier L, Ge Y, Gentry J, Hornik K, Hothorn T, Huber W, lacus S, Irizarry R, Leisch F, Li C, Maechler M, Rossini AJ, Sawitzki G, Smith C, Smyth G, Tierney L, Yang JY, Zhang J (2004) Bioconductor: open software development for computational biology and bioinformatics. Genome biology 5: R80

Gould KS (2004) Nature's Swiss Army Knife: The Diverse Protective Roles of Anthocyanins in Leaves. Journal of biomedicine \& biotechnology 2004: 314-320

Graham TL (1991) Flavonoid and Isoflavonoid Distribution in Developing Soybean Seedling Tissues and in Seed and Root Exudates. Plant Physiol 95: 594-603

Grotewold E (2006) The genetics and biochemistry of floral pigments. Annual review of plant biology 57: 761-780

Grotewold E, Davis K (2008) Trafficking and sequestration of anthocyanins. Nat. Prod. Comm. 3: 1251-1258

Hahlbrock K, Knobloch K, Kreuzaler F, Potts J, Wellmann E (1976) Coordinated induction and subsequent activity changes of two groups of metabolically interrelated enzymes. Light-induced synthesis of Xavonoid glycosides in cell suspension cultures of Petroselinum hortense. Eur $\mathrm{J}$ Biochem 61: 199-206

Hartmann U, Sagasser M, Mehrtens F, Stracke R, Weisshaar B (2005) Differential combinatorial interactions of cis-acting elements recognized by R2R3-MYB, BZIP, and BHLH factors control light-responsive and tissuespecific activation of phenylpropanoid biosynthesis genes. Plant molecular biology 57: 155-171

Heller W, Forkmann G (1988) Biosynthesis of flavonoids. In JB Harborne, ed, The Flavonoids: Advances in Research Since 1980. Chapman \& Hall, London, pp 399-425

Heller W, Forkmann G (1993) Biosynthesis of flavonoids. In JB Harborne, ed, The Flavonoids: Advances in Research Since 1986. Chapman \& Hall, London, pp 499-535 
Hertog MGL, Hollman P, Katan MP (1992) Content of potentially anticarcenogenic flavonoids of 28 vegetables and 9 fruits commonly consumed in the Netherlands. J Agric Food Chem 40: 2379-2383.

Hirai MY, Klein M, Fujikawa Y, Yano M, Goodenowe DB, Yamazaki Y, Kanaya S, Nakamura Y, Kitayama M, Suzuki H, Sakurai N, Shibata D, Tokuhisa J, Reichelt M, Gershenzon J, Papenbrock J, Saito K (2005) Elucidation of gene-to-gene and metabolite-to-gene networks in arabidopsis by integration of metabolomics and transcriptomics. The Journal of biological chemistry 280: 25590-25595

Hirai MY, Yano M, Goodenowe DB, Kanaya S, Kimura T, Awazuhara M, Arita M, Fujiwara T, Saito K (2004) Integration of transcriptomics and metabolomics for understanding of global responses to nutritional stresses in Arabidopsis thaliana. Proceedings of the National Academy of Sciences of the United States of America 101: 10205-10210

Holton T, Tanaka Y (1994) Blue roses-a pigment of our imagination? Trends Biotechnol 12: 40-42

Holton TA (1995) Modification of flower colour via manipulation of P450 gene expression in transgenic plants. Drug metabolism and drug interactions 12: $359-368$

Holton TA, Brugliera F, Tanaka Y (1993) Cloning and expression of flavonol synthase from Petunia hybrida. The Plant journal : for cell and molecular biology 4: 1003-1010

Hostel W (1981). In W Stumpf, PM Conn, eds, The Biochemistry of Plants, Vol 7. Academic Press, New York, pp 725-753

Hughes J, Hughes MA (1994) Multiple secondary plant product UDP-glucose glucosyltransferase genes expressed in cassava (Manihot esculenta Crantz) cotyledons. DNA Seq 5: 41-49

Irizarry RA, Hobbs B, Collin F, Beazer-Barclay YD, Antonellis KJ, Scherf U, Speed TP (2003) Exploration, normalization, and summaries of high density oligonucleotide array probe level data. Biostatistics (Oxford, England) 4: 249-264

Ivashuta SI, Petrick JS, Heisel SE, Zhang Y, Guo L, Reynolds TL, Rice JF, Allen E, Roberts JK (2009) Endogenous small RNAs in grain: Semiquantification and sequence homology to human and animal genes. Food and Chemical Toxicology 47: 353-360

Iwashina T, Githiri SM, Benitez ER, Takemura T, Kitajima J, Takahashi R (2007) Analysis of flavonoids in flower petals of soybean near-isogenic lines for flower and pubescence color genes. J Hered 98: 250-257

Karg SR, Kallio PT (2009) The production of biopharmaceuticals in plant systems. Biotechnology advances 27: 879-894

Katsumoto Y, Fukuchi-Mizutani M, Fukui Y, Brugliera F, Holton TA, Karan M, Nakamura N, Yonekura-Sakakibara K, Togami J, Pigeaire A, Tao GQ, Nehra NS, Lu CY, Dyson BK, Tsuda S, Ashikari T, Kusumi T, Mason JG, Tanaka Y (2007) Engineering of the rose flavonoid 
biosynthetic pathway successfully generated blue-hued flowers accumulating delphinidin. Plant \& cell physiology 48: 1589-1600

Kennedy JA, Jones GP (2003) Analysis of proanthocyanidin cleavage products following acid-catalysis in the presence of excess phloroglucinol. J Agric Food Chem 49: 1740-1746

Kovinich N, Arnason JT, DeLuca V, Miki B (2011a) Coloring Soybeans with Anthocyanins? In DR Gang, ed, Recent Advances in Phytochemistry, Vol 41. Springer, pp 47-57

Kovinich N, Saleem A, Arnason JT, Miki B (2010) Functional characterization of a UDP-glucose:flavonoid 3-O-glucosyltransferase from the seed coat of black soybean (Glycine max (L.) Merr.). Phytochemistry 71: 1253-1263

Kovinich N, Saleem A, Arnason JT, Miki B (2011b) Combined analysis of transcriptome and metabolite data reveals extensive differences between black and brown nearly-isogenic soybean (Glycine max) seed coats enabling the identification of pigment isogenes. 12: 381. doi:10.1186/1471-2164-12-381. Kovinich N, Saleem A, Arnason JT, Miki B (2011c) Identification of two anthocyanidin reductase genes from the seed coat of brown soybean and three red-brown soybean accessions that have reduced

ANTHOCYANIDIN REDUCTASE1 mRNA, activity, and seed coat proanthocyanidin amounts. Submitted to Plant phys and biochem

Kovinich N, Saleem A, Arnason JT, and Miki B. (2011d). Aposomatic GM soybeans: Coloring genetically modified soybean grains with anthocyanins by suppression of the proanthocyanidin genes ANR1 and ANR2. Submitted to Transgenic research

Krueger R, Le Buanec B (2008) Action needed to harmonize regulation of lowlevel presence of biotech traits. Nature biotechnology 26: 161-162

Ku H-M (2009) Molecular cloning and expression of ANS ( anthocyanidin synthase ) and GST ( glutathione S-transferase ) in seed coats of soybean. National Chung Hsing University, Taichung

Kubo H, Nawa N, Lupsea SA (2007) Anthocyaninless1 gene of Arabidopsis thaliana encodes a UDP-glucose:flavonoid-3-O-glucosyltransferase. J Plant Res 120: 445-449

Lee JH, Kang NS, Shin S-O, Shin S-H, Lim S-G, Suh D-Y, Baek I-Y, Park K-Y, Ha TJ (2009) Characterization of anthocyanins in the black soybean (Glycine max L.) by HPLC-DAD-ESI/MS analysis. Food Chemistry 112: 226-231

Lee Y, Yoon H, Paik YS, Liu JR, Chung W-I, Choi G (2005) Reciprocal regulation of Arabidopsis UGT78D2 and BANYULS is critical for regulation of the metabolic flux of anthocyanidins to condensed tannins in developing seed coats. Journal of Plant Biology 48: 356-370

Lemaux PG (2008) Genetically Engineered Plants and Foods: A Scientist's Analysis of the Issues (Part I). Annual review of plant biology 59: 771-812 
Lepiniec L, Debeaujon I, Routaboul JM, Baudry A, Pourcel L, Nesi N, Caboche M (2006) Genetics and biochemistry of seed flavonoids. Annu Rev Plant Biol 57: 405-430

Li Y-G, Tanner G, Larkin P (1996) The DMACA-HC1 Protocol and the Threshold Proanthocyanidin Content for Bloat Safety in Forage Legumes. J. Agric. Food Chem. 70: 89-101

Li Y, Baldauf S, Lim EK, Bowles DJ (2001) Phylogenetic analysis of the UDPglycosyltransferase multigene family of Arabidopsis thaliana. J Biol Chem 276: 4338-4343

Liu H, Du Y, Chu H, Shih CH, Wong YW, Wang M, Chu IK, Tao Y, Lo C (2010) Molecular dissection of the pathogen-inducible 3-deoxyanthocyanidin biosynthesis pathway in sorghum. Plant \& cell physiology 51: 1173-1185

Loake GJ, Choudhary AD, Harrison MJ, Mavandad M, Lamb CJ, Dixon RA (1991) Phenylpropanoid pathway intermediates regulate transient expression of a chalcone synthase gene promoter. The Plant cell 3: 829840

Mackenzie PI, Owens IS, Burchell B, Bock KW, Bairoch A, Belanger A, Fournel-Gigleux S, Green M, Hum DW, lyanagi T, Lancet D, Louisot $P$, Magdalou J, Chowdhury JR, Ritter JK, Schachter H, Tephly TR, Tipton KF, Nebert DW (1997) The UDP glycosyltransferase gene superfamily: recommended nomenclature update based on evolutionary divergence. Pharmacogenetics 7: 255-269

Malik K, Wu K, Li XQ, Martin-Heller T, Hu M, Foster E, Tian L, Wang C, Ward K, Jordan M, Brown D, Gleddie S, Simmonds D, Zheng S, Simmonds J, Miki B (2002) A constitutive gene expression system derived from the tCUP cryptic promoter elements. Theor Appl Genet 105: 505-514

Mancinelli AL (1990) Interaction between Light Quality and Light Quantity in the Photoregulation of Anthocyanin Production. Plant Physiol 92: 1191-1195

Marrs KA, Alfenito MR, Lloyd AM, Walbot V (1995) A glutathione Stransferase involved in vacuolar transfer encoded by the maize gene Bronze-2. Nature 375: 397-400

Martin A, Cabrera A, Lopez-Medina J (1995) Tannin content in Vicia faba: possibilities for plant breeding. Options Méditerranéennes 10: 105-110

McCallum JA, Walker JRL (1990) Proanthocyanidins in wheat bran. Cereal Chemistry 67: 282-285

Meyer P, Heidmann I, Forkmann G, Saedler H (1987) A new petunia flower colour generated by transformation of a mutant with a maize gene. Nature 330: $677-678$

Miki D, Shimamoto K (2004) Simple RNAi vectors for stable and transient suppression of gene function in rice. Plant \& cell physiology 45: 490-495

Modolo LV, Blount JW, Achnine L, Naoumkina MA, Wang X, Dixon RA (2007) A functional genomics approach to (iso)flavonoid glycosylation in the model legume Medicago truncatula. Plant Mol Biol 64: 499-518 
Mol J, Grotewold E, Koesa R (1998) How genes paint flowers and seeds Trends in Plant Science 3: 212-217

Morisset D, Demsar T, Gruden K, Vojvoda J, Stebih D, Zel J (2009) Detection of genetically modified organisms--closing the gaps. Nature biotechnology 27: $700-701$

Naczk M, Nichols T, Pink D, Sosulski F (1994) Condensed Tannins in Canola Hulls. J. Agric. Food Chem. 42: 2196-2200

Nagai I (1921) A genetico-physiological study of the formation of anthocyanins and brown pigments in plant. Tokyo Univ Coll Agric J 8: 1-92

Nagamatsu A, Masuta C, Matsuura H, Kitamura K, Abe J, Kanazawa A (2009) Down-regulation of flavonoid 3'-hydroxylase gene expression by virus-induced gene silencing in soybean reveals the presence of a threshold mRNA level associated with pigmentation in pubescence. Journal of plant physiology 166: 32-39

Nakatsuka T, Abe Y, Kakizaki Y, Yamamura S, Nishihara M (2007) Production of red-flowered plants by genetic engineering of multiple flavonoid biosynthetic genes. Plant cell reports 26: 1951-1959

Nishihara M, Nakatsuka T (2011) Genetic engineering of flavonoid pigments to modify flower color in floricultural plants. Biotechnology letters 33: 433-441

Offen W, Martinez-Fleites C, Yang M, Kiat-Lim E, Davis BG, Tarling CA, Ford CM, Bowles DJ, Davies GJ (2006) Structure of a flavonoid glucosyltransferase reveals the basis for plant natural product modification. EMBO J 25: 1396-1405

Ogata J, Itoh Y, Ishida M, Yoshida H, Ozeki Y (2004) Cloning and heterologous expression of cDNAs encoding flavonoid glucosyltransferases from Dianthus caryophyllus. Plant Biotechnology 21: $367-375$

Ogata J, Teramoto S, Yoshitama K (1998) Isolation and characterization of UDP-glucose: cyanidin 3-O-glucosyltransferase from the flower buds of Senecio x hybridus. J. Plant Res. 111: 213-216

Osmani SA, Bak S, Moller BL (2009) Substrate specificity of plant UDPdependent glycosyltransferases predicted from crystal structures and homology modeling. Phytochemistry 70: 325-347

Owens DK, McIntosh CA (2009) Identification, recombinant expression, and biochemical characterization of a flavonol 3-O-glucosyltransferase clone from Citrus paradisi. Phytochemistry 70: 1382-1391

Pang Y, Peel GJ, Wright E, Wang Z, Dixon RA (2007) Early steps in proanthocyanidin biosynthesis in the model legume Medicago truncatula. Plant Physiol 145: 601-615

Paolocci F, Robbins MP, Madeo L, Arcioni S, Martens S, Damiani F (2007) Ectopic expression of a basic helix-loop-helix gene transactivates parallel pathways of proanthocyanidin biosynthesis. structure, expression analysis, and genetic control of leucoanthocyanidin 4-reductase and 
anthocyanidin reductase genes in Lotus corniculatus. Plant Physiol 143: 504-516

Peel GJ, Pang Y, Modolo LV, Dixon RA (2009) The LAP1 MYB transcription factor orchestrates anthocyanidin biosynthesis and glycosylation in Medicago. Plant J 59: 136-149

Pelletier MK, Murrell JR, Shirley BW (1997) Characterization of flavonol synthase and leucoanthocyanidin dioxygenase genes in Arabidopsis. Further evidence for differential regulation of "early" and "late" genes. Plant physiology 113: 1437-1445

Pienkny S, Brandt W, Schmidt J, Kramell R, Ziegler J (2009) Functional characterization of a novel benzylisoquinoline O-methyltransferase suggests its involvement in papaverine biosynthesis in opium poppy (Papaver somniferum L). The Plant journal : for cell and molecular biology 60: $56-67$

Price ML, Hagerman AE, Butler LG (1980) Tannin content of cowpeas, chickpeas, pigeon peas, and mung beans. J. Agric. Food Chem. 28: 459461

Ralston EJ, English JJ, Dooner HK (1988) Sequence of three bronze alleles of maize and correlation with the genetic fine structure. Genetics 119: 185197

Ramessar K, Capell T, Twyman RM, Christou P (2010) Going to ridiculous lengths--European coexistence regulations for GM crops. Nature biotechnology 28: 133-136

Ranathunge K, Shao S, Qutob D, Gijzen M, Peterson CA, Bernards MA (2010) Properties of the soybean seed coat cuticle change during development. Planta 231: 1171-1188

Ross J, Li Y, Lim E, Bowles DJ (2001) Higher plant glycosyltransferases. Genome Biol 2: REVIEWS3004

Routaboul JM, Kerhoas L, Debeaujon I, Pourcel L, Caboche M, Einhorn J, Lepiniec $L$ (2006) Flavonoid diversity and biosynthesis in seed of Arabidopsis thaliana. Planta 224: 96-107

Richards HA, Han C-T, Hopkins RG, Failla ML, Ward WW, Stewart CN Jr (2003) Safety Assessment of Recombinant Green Fluorescent Protein Orally Administered to Weaned Rats. J Nutr 133: 1909-1912

Saleh NAM, Fritsch H, Witkop P, Grisebach H (1976) UDP-glucose: cyanidin 3-O-glucosyltransferase from cell cultures of Haplopappus gracilis. Planta 133: $41-45$

Saleh NAM, Poulton J, Gris-Bach H (1976) UDP-glucose: cyanidin 3-Oglucosyltransferase grom cabbage seedlings. Phytochemistry 15: 1865-1868

Sarkar TR, Strong CL, Sibhatu MB, Pike LM, McIntosh CA (2007) Cloning expression and characterization of a putative flavonoid glucosyltransferase from grapefruit (Citrus paradisi) leaves. In N B.J., W E.S., eds, Concepts in Plant Metabolomics. Springer, Dordrecht, pp 247257 
Saslowsky D, Winkel-Shirley B (2001) Localization of flavonoid enzymes in Arabidopsis roots. Plant J 27: 37-48

Schmutz J, Cannon SB, Schlueter J, Ma J, Mitros T, Nelson W, Hyten DL, Song Q, Thelen JJ, Cheng J, Xu D, Hellsten U, May GD, Yu Y, Sakurai T, Umezawa T, Bhattacharyya MK, Sandhu D, Valliyodan B, Lindquist E, Peto M, Grant D, Shu S, Goodstein D, Barry K, Futrell-Griggs M, Abernathy B, Du J, Tian Z, Zhu L, Gill N, Joshi T, Libault M, Sethuraman A, Zhang XC, Shinozaki K, Nguyen HT, Wing RA, Cregan P, Specht J, Grimwood J, Rokhsar D, Stacey G, Shoemaker RC, Jackson SA (2010) Genome sequence of the palaeopolyploid soybean. Nature 463: 178-183

Shen L, Petolino J (2006) Pigmented maize seed via tissue-specific expression of anthocyanin pathway gene transcription factors. Mol Breeding 18: $57-$ 67

Smyth S, McHughen A (2008) Regulating innovative crop technologies in Canada: the case of regulating genetically modified crops. Plant biotechnology journal 6: 213-225

Snyder BA, Nicholson RL (1990) Synthesis of phytoalexins in sorghum as a site-specific response to fungal ingress. Science (New York, N.Y.) 248: 1637-1639

Song QJ, Marek LF, Shoemaker RC, Lark KG, Concibido VC, Delannay X, Specht JE, Cregan PB (2004) A new integrated genetic linkage map of the soybean. TAG. Theoretical and applied genetics. Theoretische und angewandte Genetik 109: 122-128

Soy 20/20 (2010) Canada's-soy-value-chain. http://www.soy2020.ca/pdfs/ Canadas-Soybean-Value-Chain.pdf

Stewart CN, Jr. (2005) Monitoring the presence and expression of transgenes in living plants. Trends in plant science 10: 390-396

Stewart CN, Jr. (2006) Go with the glow: fluorescent proteins to light transgenic organisms. Trends in biotechnology 24: 155-162

Stracke R, De Vos RC, Bartelniewoehner L, Ishihara H, Sagasser M, Martens S, Weisshaar B (2009) Metabolomic and genetic analyses of flavonol synthesis in Arabidopsis thaliana support the in vivo involvement of leucoanthocyanidin dioxygenase. Planta 229: 427-445

Stracke R, Ishihara H, Huep G, Barsch A, Mehrtens F, Niehaus K, Weisshaar B (2007) Differential regulation of closely related R2R3-MYB transcription factors controls flavonol accumulation in different parts of the Arabidopsis thaliana seedling. The Plant journal : for cell and molecular biology 50: $660-677$

Sugimoto T, Kawasaki T, Kato T, Whittier RF, Shibata D, Kawamura Y (1992) cDNA sequence and expression of a phosphoenolpyruvate carboxylase gene from soybean. Plant molecular biology 20: 743-747

Tanaka Y, Brugliera F, Chandler S (2009) Recent progress of flower colour modification by biotechnology. International journal of molecular sciences 10: $5350-5369$ 
Tanaka Y, Katsumoto Y, Brugliera F, Mason J (2005) Genetic engineering in floriculture. . Plant Cell, Tiss and Org Cult 80: 1-24

Tanaka Y, Tsuda S, Kusumi T (1998) Metabolic Engineering to Modify Flower Color. Plant Cell Physiol 39: 1119-1126

Tanaka Y, Yonekura K, Fukuchi-Mizutani M, Fukui Y, Fujiwara H, Ashikari T, Kusumi T (1996) Molecular and biochemical characterization of three anthocyanin synthetic enzymes from Gentiana triflora. Plant Cell Physiol 37: 711-716

Tanner GJ, Francki KT, Abrahams S, Watson JM, Larkin PJ, Ashton AR (2003) Proanthocyanidin biosynthesis in plants. Purification of legume leucoanthocyanidin reductase and molecular cloning of its cDNA. J Biol Chem 278: 31647-31656

Toda K, Yang D, Yamanaka N, Watanabe S, Harada K, Takahashi R (2002) A single-base deletion in soybean flavonoid 3'-hydroxylase gene is associated with gray pubescence color. Plant molecular biology 50: 187196

Todd J, Vodkin L (1996) Duplications That Suppress and Deletions That Restore Expression from a Chalcone Synthase Multigene Family. The Plant cell 8: 687-699

Todd JJ, Vodkin LO (1993) Pigmented Soybean (Glycine max) Seed Coats Accumulate Proanthocyanidins during Development. Plant physiology 102: $663-670$

Tohge T, Nishiyama Y, Hirai MY, Yano M, Nakajima J, Awazuhara M, Inoue E, Takahashi H, Goodenowe DB, Kitayama M, Noji M, Yamazaki M, Saito K (2005) Functional genomics by integrated analysis of metabolome and transcriptome of Arabidopsis plants over-expressing an MYB transcription factor. Plant J 42: 218-235

Tsuda S, Fukui Y, Nakamura N, Katsumoto Y, Yonekura-Sakakibara K, Fukuchi-Mizutani M, Ohira K, Ueyama Y, Ohkawa H, Holton TA, Kusumi T, Tanaka Y (2004) Flower color modification of Petunia hybrida commercial varieties by metabolic engineering. Plant Biotechnology 21: 377-386

Tuteja JH, Clough SJ, Chan WC, Vodkin LO (2004) Tissue-specific gene silencing mediated by a naturally occurring chalcone synthase gene cluster in Glycine max. The Plant cell 16: 819-835

Venglat P, Xiang D, Qiu S, Stone SL, Tibiche S, Cram D, Alting-Mees M, Nowak J, Cloutier S, Deyholos M, Bekkaoui F, Sharpe A, Wang E, Rowland G, Selvaraj G, Datla R (2011) Gene expression analysis of flax seed development. BMC Plant Biology 11:74 doi:10.1186/1471-2229-1174

Vogt T, Jones P (2000) Glycosyltransferases in plant natural product synthesis: characterization of a supergene family. Trends Plant Sci 5: 380-386

Wang C-S, Vodkin LO (1994) Extraction of RNA from tissues containing high levels of procyanidins that bind RNA. Plant Molecular Biology Reporter 12: 132-145 
Wang CS, Todd JJ, Vodkin LO (1994) Chalcone synthase mRNA and activity are reduced in yellow soybean seed coats with dominant I alleles. Plant physiology 105: 739-748

Wang $X$ (2009) Structure, mechanism and engineering of plant natural product glycosyltransferases. FEBS Lett 583: 3303-3309

Weiss M (1970) Genetic linkage in soybeans. Linkage group VII. Crop Sci 10: $627-629$

Winkel-Shirley B (1998) Flavonoids in seeds and grains: Physiological function, agronomic importance and the genetics of biosynthesis. Seed Science Research 8: 415-422

Winkel-Shirley B (2001) Flavonoid biosynthesis. A colorful model for genetics, biochemistry, cell biology, and biotechnology. Plant physiology 126: 485493

Winkel BSJ (2009) Metabolite channeling and multi-enzyme complexes. In AE Osbourn, V Lanzotti, eds, Plant-derived natural products. Springer New York, pp 195-208

Woodworth CM (1921) Inheritance of Cotyledon, Seed-Coat, Hilum and Pubescence Colors in Soy-Beans. Genetics 6: 487-553

Xie DY, Sharma SB, Paiva NL, Ferreira D, Dixon RA (2003) Role of anthocyanidin reductase, encoded by BANYULS in plant flavonoid biosynthesis. Science 299: 396-399

Yang K, Jeong N, Moon JK, Lee YH, Lee SH, Kim HM, Hwang CH, Back K, Palmer RG, Jeong SC (2010) Genetic analysis of genes controlling natural variation of seed coat and flower colors in soybean. The Journal of heredity 101: 757-768

Yoshihara N, Imayama T, Fukuchi-Mizutani M, Okuhara H, Tanaka Y, Ino I, Yabuya T (2005) cDNA cloning and characterization of UDP-glucose: anthocyanidin 3-O-glucosyltransferase in Iris hollandica. Plant Science 169: 496-501

Yu O, Shi J, Hession AO, Maxwell CA, McGonigle B, Odell JT (2003) Metabolic engineering to increase isoflavone biosynthesis in soybean seed. Phytochemistry 63: 753-763

Zabala G, Vodkin L (2003) Cloning of the pleiotropic T locus in soybean and two recessive alleles that differentially affect structure and expression of the encoded flavonoid 3' hydroxylase. Genetics 163: 295-309

Zabala G, Vodkin LO (2005) The wp mutation of Glycine max carries a genefragment-rich transposon of the CACTA superfamily. The Plant cell 17: 2619-2632

Zabala G, Vodkin LO (2007) A rearrangement resulting in small tandem repeats in the F3'5' $\mathrm{H}$ gene of white flower genotypes is associated with the Soybean W1 locus. The Plant Genome 47: S113-S124

Zhao J, Dixon RA (2010) The 'ins' and 'outs' of flavonoid transport. Trends in plant science 15: $72-80$

Zhao J, Huhman D, Shadle G, He XZ, Sumner LW, Tang Y, Dixon RA (2011) MATE2 Mediates Vacuolar Sequestration of Flavonoid Glycosides and 
Glycoside Malonates in Medicago truncatula. The Plant cell 23: 15361555

\section{APPENDIX A}

\begin{tabular}{|c|c|c|c|c|}
\hline Experıment & Corresponding probe set & Target & Prımer & Sequence $\left(5^{\prime} \text { to } 3^{\prime}\right)^{\dagger \neq}$ \\
\hline \multirow[t]{38}{*}{$\begin{array}{l}\text { Seml-qRT- } \\
\text { PCR }\end{array}$} & GmaAffx 39483 1 A1_at & MYB159 & $159 \mathrm{~F}$ & TGACTCAGCCTCACCACTTG \\
\hline & & & $159 \mathrm{R}$ & CAAAGGCATTGACCCAAATAA \\
\hline & Gma 15972 1 A1_at & $4 \mathrm{CL} 2$ & $4 \mathrm{C} 2 \mathrm{~F}$ & ACAAACATGGTGGTGGACAA \\
\hline & & & $4 \mathrm{C} 2 \mathrm{R}$ & GGTAGAAACCGCTGCCACT \\
\hline & Gma 7423 2 S1_a_at & $4 \mathrm{CL}$ & $4 \mathrm{CF}$ & GCAAGACCAATCTTGCATCC \\
\hline & & & $4 \mathrm{CR}$ & TTTTGCCCAGGCATATATGTAA \\
\hline & Gma 2605 1 S1_at & $9012 b$ & $901 \mathrm{~F}$ & CACTTTGACGTCGATCCAAAC \\
\hline & & & $901 \mathrm{R}$ & GAATCAGATGTCAAACCGTTCA \\
\hline & Gma 26052 S1_at & $9012 a$ & $902 \mathrm{~F}$ & GCACGAGGGAAAATGTGTTT \\
\hline & & & $902 \mathrm{R}$ & GTTGGAAACGGATTTGGTGT \\
\hline & & ANR1 & qAF & СССТСААТАТАAАATTССАAСТGAA \\
\hline & & & qAR & CAGAGCCCCTTTGCTCTTTA \\
\hline & GmaAffx 421161 S1_at & CHS4 & CHS4F & ССTTCCAAGCCACTTTGCA \\
\hline & & & CHS4R & CTGGAGCAAAGGATGAAAGTGA \\
\hline & GmaAffx 42116 1S1_at & CHS5 & CHS5F & CACTTTGCCACATTCATTCC \\
\hline & & & CHS5R & TGTGAATGAACTAATGAAGCTATAGC \\
\hline & & $\begin{array}{l}\text { CHS7I } \\
\text { CHS8 }\end{array}$ & CHS78F & TAGGCAAGACATGGTGGTGG \\
\hline & & & CHS78R & CTTTGACTTTGGCTGGCCC \\
\hline & GmaAffx 807201 S1_at & DFR2 & DFR2F & ССССТТТТТАТGССAACAA \\
\hline & & & DFR2R & ATGTACCTCCCTTCGGCTTT \\
\hline & Gma 15972 1A1_at & EF-Hand & EHF & ACGCCATCTCCATTCAAATC \\
\hline & & & EHR & CAGCAGGGGAAGATGAGAAG \\
\hline & Gma 5139 1 S1_at & GST 21 & G21F & ACTTGATGCAGACCCCTTGT \\
\hline & & & G21R & TTCAAGAACACCCAACACCA \\
\hline & GmaAffx 71212 1 A1_at & GST1 & GST1F & CCAGGGCTTGAACATCTCAT \\
\hline & & & GST1R & TCCACGCCATGTATTTGTTG \\
\hline & GmaAffx 90491 1 A1_s_at & $\begin{array}{l}\text { Proteın } \\
\text { kınase }\end{array}$ & KIF & GAAAGGTTTGAACGCACCAT \\
\hline & & & KIR & ATITTGGCGGAGTTTCACAC \\
\hline & GmaAffx 732732 S1_s_at & Lipase & LIPF & CCGCATGGTTCTATCTTGGT \\
\hline & & & LIPR & TGCATTTGACAAACCCTTCA \\
\hline & GmaAffx 45454 1 S1_at & LRR & LRF & TTACATCAATGGCGGGATCT \\
\hline & & & LRR & TTGGTGGGAACTTCTTITGG \\
\hline & GmaAffx 81605 1 S1_at & MYB50 & M50F & TTTCAATCTGGAATTGGTTCG \\
\hline & & & M50R & ТТТАСТСАТТСААССААТАAСАACAC \\
\hline & GmaAffx 57777 1 S1_at & OMT5 & $\mathrm{OMT} 2 \mathrm{~F}$ & ССТСACTGCACTTACCATTCC \\
\hline & & & OMT2R & TCTTTATCGGCATCAACGAA \\
\hline & Gma 9647 1 S1_at & OMT-lıke & OMTF & TTTGTTGAAAGCCAGTGCAG \\
\hline & & & OMTR & TITIGCCTTTGTTGATGCTG \\
\hline
\end{tabular}




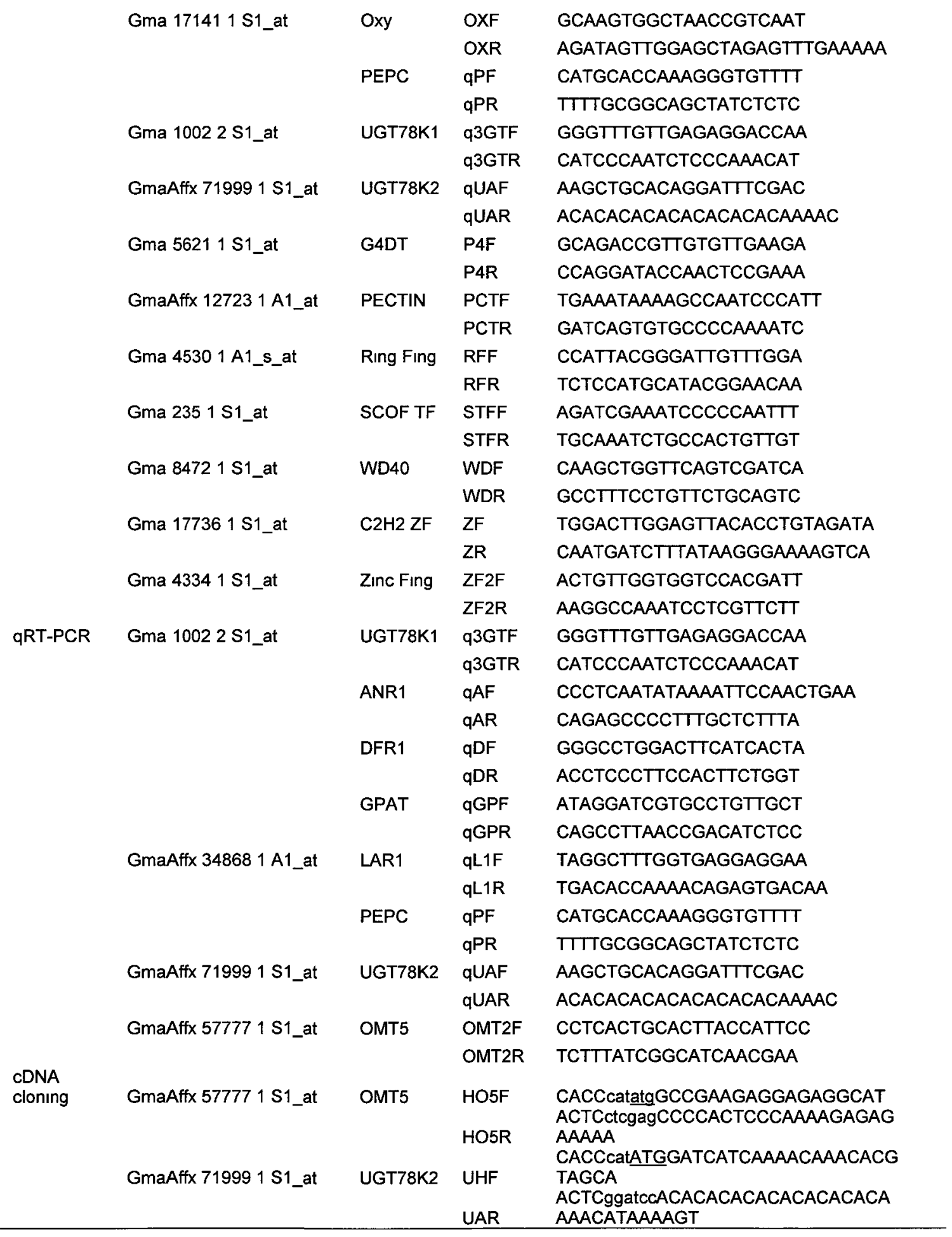

${ }^{\dagger}$ Restriction site in lowercase

${ }^{\ddagger}$ Start codon underlıned 


\section{APPENDIX B}

Developmental properties of black (iRT) and brown (irT) soybean seed coats at the $300-$ $400 \mathrm{mg}$ fresh seed weight stage

\begin{tabular}{lccc}
\hline Property & Black $(i R T)$ & Brown (irT) & $\begin{array}{c}\text { P- } \\
\text { value }\end{array}$ \\
\hline Days post-anthesis & 30 & 30 & $\mathrm{~N} / \mathrm{A}$ \\
Pod length $(\mathrm{cm})^{\mathrm{b}}$ & $5.019 \pm 0.20$ & $5.206 \pm 0.35$ & 0.214 \\
Pod color & yellowish-green & $\begin{array}{c}\text { yellowish-green } \\
\text { transition/early }\end{array}$ & $\mathrm{N} / \mathrm{A}$ \\
Embryo morphology & transition/early maturation & maturation & $\mathrm{N} / \mathrm{A}$ \\
GPAT relative expression & $0.512 \pm 0.23$ & $0.510 \pm 0.17$ & 0.944 \\
DFR1 relative expression & $0.601 \pm 0.24$ & $0.491 \pm 0.06$ & 0.220 \\
\hline
\end{tabular}

a'Student's t test.

${ }^{b}$ Pods containing three seeds.

cqRT-PCR expression levels relative to PEPC16. 
APPENDIX C

A
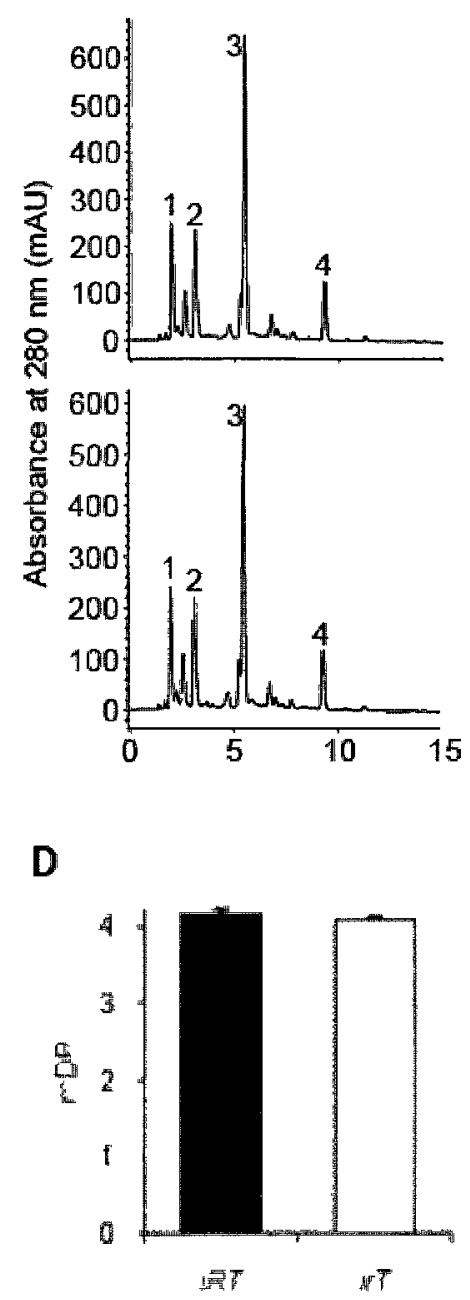

B
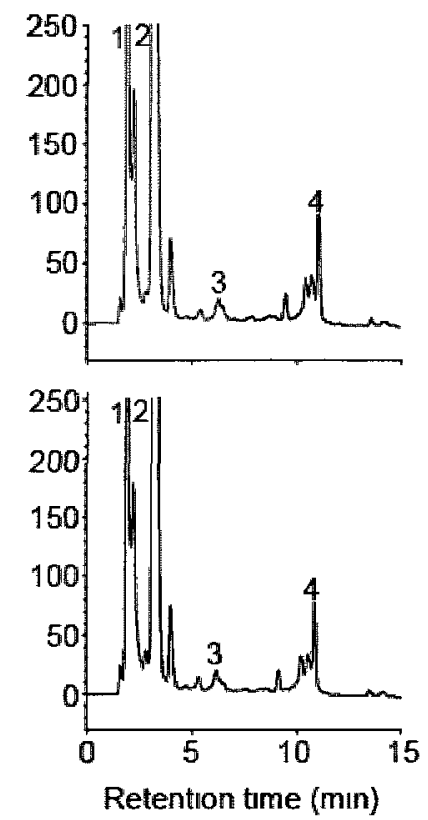

E

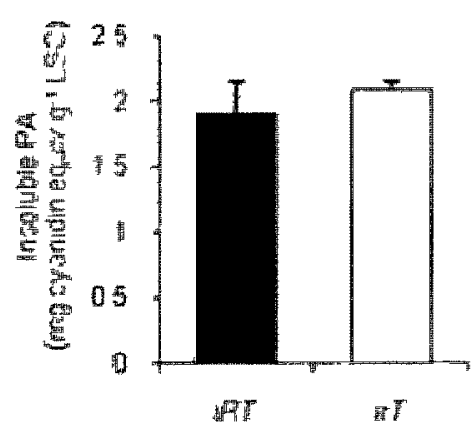

C
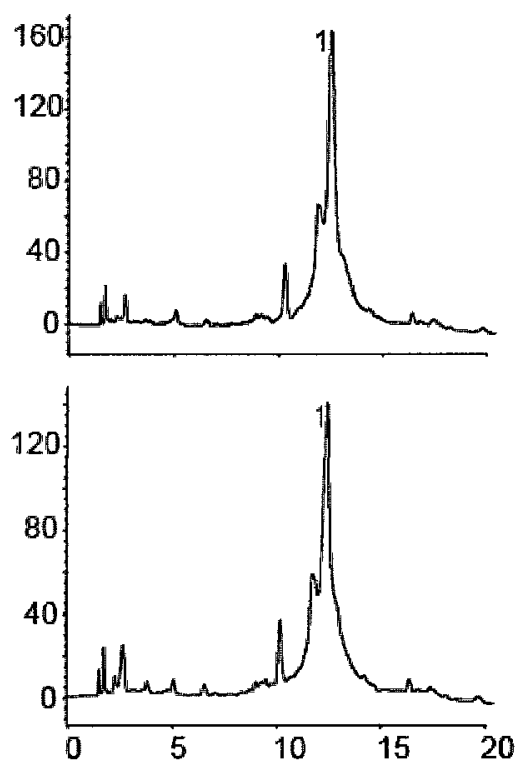

F

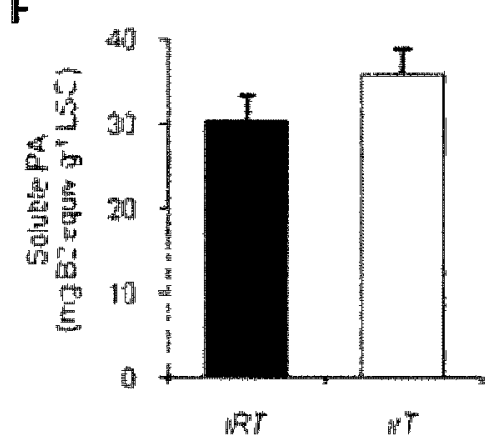

Proanthocyanidin (PA) subunit compositions, degree of polymerizations, and amounts from the seed coats of black (iRT) and brown (irT) soybean Clark isolines. $(\mathrm{A}, \mathrm{B}, \mathrm{C})$ iRT top panels, irT bottom panels. (A) Phloroglucinol cleavage products of soluble PA polymers. HPLC retention times: ascorbic acid (1) (Rt: 2.0 min); phloroglucinol (2) (Rt: $3.1 \mathrm{~min}$ ); epicatechin-phloroglucinol adduct (Rt: 5.5 min); epicatechin (4) (Rt: $9.4 \mathrm{~min})$. (B) Phloroglucinol cleavage products of solvent insoluble PA polymers. HPLC retention times: ascorbic acid (1) (Rt: 2.0 min); phloroglucinol (2) (Rt: $3.1 \mathrm{~min}$ ); epicatechin-phloroglucinol (Rt: $6.2 \mathrm{~min}$ ); epicatechin (4) (Rt: $11.0 \mathrm{~min}$ ). (C) Free monomers. HPLC retention times: epicatechin (1) (Rt: $12.5 \mathrm{~min}$ ). (D) Mean degree of polymerization (mDP) of soluble PAs. (E) Total insoluble PAs. (F) Total soluble PAs. (D, E, F) iRT black bars, irT white bars. (E, D) Amounts are represented as milligrams procyanidin B2 equivalents per gram lyophilized seed coat (LSC). 


\section{APPENDIX D}

Gene probe sets up-regulated more than 2-fold in the seed coat of black (IRT) soybean compared to the brown (IrT) isoline ${ }^{a}$

\begin{tabular}{|c|c|c|c|c|}
\hline \multirow{2}{*}{ Probe Sets } & \multirow{2}{*}{ Target Description } & \multirow{2}{*}{$\begin{array}{l}\text { Black/brown } \\
\text { fold change }\end{array}$} & \multicolumn{2}{|c|}{ SAM } \\
\hline & & & P-value & d-score \\
\hline GmaAffx 71212 1A1_at & Glutathıone S-transferase (GST26) & 23411 & 0000003 & 2122 \\
\hline GmaAffx 80720 1 S1_at & Dihydroflavonol-4-reductase (DFR2) & 6245 & 0000002 & 2470 \\
\hline GmaAffx 42116 1 S1_at & Chalcone synthase (CHS4/CHS5) & 1099 & 0000020 & 845 \\
\hline GmaAffx 421161 S1_x_at & Chalcone synthase (CHS4/CHS5) & 806 & 0000026 & 712 \\
\hline GmaAffx 90450 1 S1_at & $\begin{array}{l}\text { Sımılar to esterase/lipase domaın } \\
(190 \mathrm{e}-44)\end{array}$ & 1041 & 0000011 & 1304 \\
\hline GmaAffx 2469 1 S1_at & $\begin{array}{l}\text { AP2/ERF transcription factor weakly } \\
\text { sImilar to DDF1 (DWARF AND } \\
\text { DELAYED FLOWERING 1) }\end{array}$ & 745 & 0001416 & 256 \\
\hline Gma 3745 1 S1_at & $\begin{array}{l}\text { Moderately sımilar to A Thaliana } \\
\text { polyamıne oxıdase (ATPAO1) } \\
\text { NP_1968741(75 6\%) }\end{array}$ & 714 & 0000028 & 711 \\
\hline Gma 1163 1 S1_at & Anthocyanıdın synthase (ANS2/ANS3) & 677 & 0000018 & 877 \\
\hline Gma 17141 1 S1_at & $\begin{array}{l}\text { Moderately sımılar to A Thaliana } \\
\text { putative flavonone 3- } \beta \text {-hydroxylase } \\
\text { clone U20744 (74\%) (F3H-lıke) }\end{array}$ & 643 & 0004849 & 203 \\
\hline GmaAffx 67044 1S1_at & $\begin{array}{l}\text { Simılar to cytochrome b5 superfamıly } \\
\text { domain }(270 \mathrm{e}-14)\end{array}$ & 586 & 0000008 & 1410 \\
\hline GmaAffx 78720 2 S1_at & $\begin{array}{l}\text { Simılar to pectınacetyltransferase } \\
\text { domain }(128 e-163)\end{array}$ & 578 & 0000057 & 520 \\
\hline Gma 10642 1 S1_at & $\begin{array}{l}\text { Similar to Mob1/phoceın superfamıly } \\
\text { domain }(334 e-45)\end{array}$ & 569 & 0000015 & 952 \\
\hline GmaAffx 732732 S1_s_at & $\begin{array}{l}\text { Similar to esterase/lıpase domain } \\
(190 \mathrm{e}-44)\end{array}$ & 529 & 0000021 & 843 \\
\hline GmaAffx 12723 1 A1_at & $\begin{array}{l}\text { Similar to leucine-rich repeat (LRR) } \\
\text { domain }(148 \mathrm{e}-03)\end{array}$ & 526 & 0000010 & 1311 \\
\hline GmaAffx 45454 1 S1_at & WD40 proteIn & 434 & 0000033 & 620 \\
\hline Gma 2605 1 S1_at & $\begin{array}{l}\text { Clone JCVI-FLGm-9012, similar to } \\
\text { uncharacterized DUF1674 domaın } \\
\text { (3 12e-13) }\end{array}$ & 402 & 0000029 & 665 \\
\hline GmaAffx 149862 S1_at & $\begin{array}{l}\text { Similar to phosphate-induced proteın } \\
\text { domain }(101 \mathrm{e}-135)\end{array}$ & 398 & 0003096 & 221 \\
\hline GmaAffx 14986 1 S1_at & $\begin{array}{l}\text { Sımılar to phosphate-induced proteın } \\
\text { domain (193E-131) }\end{array}$ & 376 & 0000915 & 284 \\
\hline Gma 17736 1 S1_at & $\begin{array}{l}\text { Moderately sımılar to A Thaliana ZAT11 } \\
\text { C2H2 type zınc finger ( } 484 \%) \\
\text { NP_1812791 }\end{array}$ & 362 & 0000379 & 357 \\
\hline Gma 26052 S1_at & $\begin{array}{l}\text { Clone JCVI-FLGm-9012, sımılar to } \\
\text { uncharacterızed DUF1674 domaın } \\
\text { (3 12e-13) }\end{array}$ & 342 & 0000064 & 503 \\
\hline Gma 17440 1S1_at & Clone $\mathrm{Gm}-\mathrm{c} 1023$, transcribed locus & 332 & 0000734 & 299 \\
\hline Gma 8411 1S1_at & $\begin{array}{l}\text { Weakly sımılar to XP_002323966 } 1 \\
\text { predicted proteın [Populus trichocarpa] }\end{array}$ & 320 & 0001318 & 260 \\
\hline Gma 4530 1 A1_s_at & $\begin{array}{l}\text { Weakly similar to A Thaliana PUB22 } \\
\text { (PLANT U-BOX 22) }(645 \%)\end{array}$ & 314 & 0000038 & 581 \\
\hline Gma 4027 1 S1_at & $\begin{array}{l}\text { Xyloglucan endotransglycosylase } \\
\text { (TCH4) }\end{array}$ & 313 & 0000281 & 390 \\
\hline GmaAffx 71999 1 S1_at & Glycosyltransferase (UGT78K2) & 309 & 0000124 & 450 \\
\hline GmaAffx 24571 1 S1_s_at & $\begin{array}{l}\text { Xyloglucan endotransglycosylase } \\
(\mathrm{TCH} 4)\end{array}$ & 305 & 0000043 & 567 \\
\hline GmaAffx 25561 S1_at & Clone Gm-r 1070 & 298 & 0009617 & 179 \\
\hline GmaAffx 62542 1S1_at & Clone Gm-r1070-2859 & 296 & 0000759 & 296 \\
\hline Psojae_rRNA_785_at & Oomycota 185 rRNA & 289 & 0000925 & 283 \\
\hline
\end{tabular}


Gma 26052 S1_a_at

Gma 10022 S1_at

Gma 74232 S1_a_at

GmaAffx 30537 1 S1_at

Gma 9672 1S1_at

GmaAffx 32504 1 A1_at

Gma $1201 \mathrm{~S} 1$ at

GmaAffx 72190 1 S1_at

Gma 4334 1 S1_at

GmaAffx 51786 1S1_s_at

GmaAffx 17284 1 A1_s_at

Gma 1171 S1_at

GmaAffx 904911 A1_s_at

Gma 9647 1 S1_at

Gma 59352 S1_a_at

Gma 3175 1S1_at

Gma 3526 1 S1_at

Gma 13307 1S1_at

GmaAffx 57777 1 S1_at

GmaAffx 41334 1 A1_at

Gma $74231 \mathrm{~S} 1 \mathrm{a}$ at

GmaAffx 18599 1 S1_at

Gma $56211 \mathrm{~S} 1$ at

Gma 8236 1 S1_at

GmaAffx 81605 1 S1_at

Gma 13607 1 S1_at

Gma 714 1 A1_at

Gma 8472 1S1_at

GmaAffx $618801 \mathrm{~S} 1$ at

Gma 3586 1 S1_at

GmaAffx 74814 1 S1_at

GmaAffx 90952 1 S1_at

GmaAffx 5393 1 S1_at

Gma 15972 1A1_at

Gma 772 1S1_at

Gma 45312 S1_at
Clone JCVI-FLGm-9O12, similar to uncharacterized DUF1674 domain

(3 12e-13)

UDP-glucose flavonold 3-O-

glucosyltransferase (UGT78K1)

4-coumarate-CoA ligase-like proteın

(4CL-like)

Phosphate-Induced protein (PHI-1)

Transcribed locus clone Gm-c1023

Lıpase (class 3 ) proteın

Syrıngolıde-ınduced proteın B13-1-9

Weakly sımılar to Formın homology 1 (AFH1) (54\%)

Zınc finger proteın, $\mathrm{C} 2 \mathrm{H} 2$ type

Xyloglucan endotransglycosylase

(TCH4)

WRKY56 transcription factor

Syrıngolide-ınduced proteın 19-1-5

Weakly sımilar to $A$ Thalıana putative serıne/threonıne proteın kınase

NP_176864 1

O-methyltransferase, putatıve (OMT-lıke)

Clone $\mathrm{Gm}-\mathrm{c} 1009-2878$, unknown proteln

Tyrosınase

Xyloglucan endotransglycosylases

(XET)

Proteın of unknown function (DUF1005)

Caffeoyl-CoA O-methyltransferase 5

(OMT5)

Clone Gm-c1079-1901

Moderately sımilar to A Thaliana AMP. dependent synthetase and ligase family proteın NP_190468 1

Polyamıne oxidase

Pterocarpan 4-dımethylallyltransferase (G4DT)

Xyloglucan endotransglycosylases

(XET)

MYB transcription factor (MYB50)

Mitochondrial oxoglutarate/malate carrier protern

Asparagınyl-tRNA synthetase

4-Coumarate-CoA ligase 2 (4CL2)

Clone Gm-r1070-3972

Unknown protein clone JCVI-FLGm-9L4

Inositol phosphate kınase (Itpk3)

Weakly sımılar to A Thalıana ubıquitınprotein ligase PLANT U-BOX 22 (PUB22) (64 5\%)

Clone JCVI-FLGm-20l13 unknown mRNA

EF-hand superfamily protein

Hypothetıcal proteın clone Gm-r1070486

Moderately sımılar to $\mathrm{A}$ Thalıana

Exordium (EXO) (80 6\%)

$\begin{array}{lll}284 & 0000262 & 396 \\ 281 & 0000229 & 403 \\ 280 & 0005815 & 196 \\ 280 & 0000309 & 382 \\ 280 & 0008550 & 183 \\ 276 & 0000046 & 554 \\ 272 & 0000935 & 283 \\ 272 & 0008756 & 182 \\ 271 & 0002176 & 235 \\ 267 & 0000152 & 434 \\ 264 & 0000708 & 303 \\ 261 & 0000154 & 434 \\ 258 & 0000765 & 296 \\ 222\end{array}$




\begin{tabular}{|c|c|c|c|c|}
\hline GmaAffx 926411 S1_s_at & Clone sHB034L03 & 216 & 0002336 & 232 \\
\hline Gma 53 1 S1_at & Maturation polypeptide (MP2) & 213 & 0005468 & 198 \\
\hline Gma 5935 1S1_at & Unknown proteın clone E01C10 & 212 & 0000173 & 426 \\
\hline Gma 5475 1 S1_at & $\begin{array}{l}\text { Weakly sımılar to A Thaliana } \\
\text { Arabınogalactan proteın } 4 \text { (AGP4) } \\
\text { NP_196605 } 1(511 \%)\end{array}$ & 211 & 0001202 & 265 \\
\hline GmaAffx 790541 S1_at & $\begin{array}{l}\text { Moderately sımilar to phosphate-induced } \\
1 \text { (PHI-1) }(783 \%)\end{array}$ & 209 & 0000240 & 400 \\
\hline GmaAffx 832132 S1_at & $\mathrm{Ca}^{2+}$-transportıng ATPase-lıke proteın & 207 & 0003554 & 215 \\
\hline Gma 5139 1 S1_at & Glutathıone S-transferase (GST21) & 205 & 0000656 & 309 \\
\hline Gma 8309 1 A1_at & Transcribed locus & 205 & 0002549 & 229 \\
\hline Gma 96111A1_at & $\begin{array}{l}\text { Moderately sımılar to A Thalıana GH3 } 1 \\
\text { (NP_179101 1) (851\%) }\end{array}$ & 203 & 0000544 & 325 \\
\hline GmaAffx 80258 1 S1_at & LEA_2 family proteın & 203 & 0001766 & 245 \\
\hline GmaAffx 84528 1 S1_at & Proteın of unknown function & 203 & 0000338 & 369 \\
\hline Gma 235 1 S1_at & Putative transcription factor SCOF-1 & 202 & 0001831 & 243 \\
\hline GmaAffx 394831 A1_at & MYB transcription factor (MYB159) & 202 & 0000034 & 617 \\
\hline Gma 235 1S1_x_at & Putative transcription factor SCOF-1 & 201 & 0001525 & 252 \\
\hline Gma 9838 1 S1_at & Thıoredoxın h1 & 200 & 0007425 & 188 \\
\hline
\end{tabular}

${ }^{a}$ Expressıon values were obtaıned usıng RMA (Irizarry et al , 2003) 


\section{Appendix E}

Distribution of gene function categories of probe sets that were up-regulated more than 2 -fold in the seed coat of black (iRT) soybean relative to the seed coat of brown (irT) soybean.

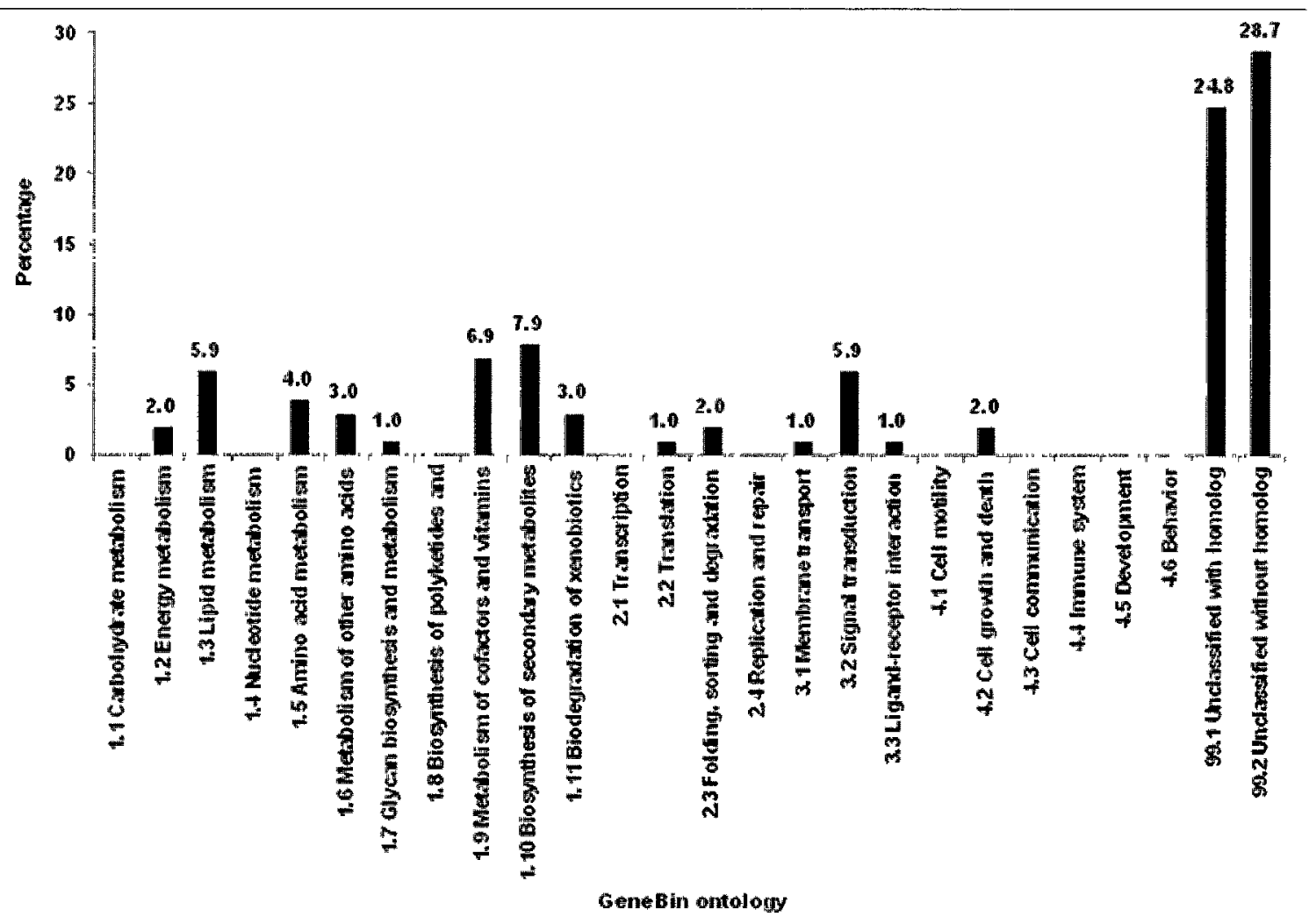




\section{Appendix F}

Glycine max UGTs ${ }^{a, b}$.

\begin{tabular}{|c|c|}
\hline Location & Gene \\
\hline \multirow[t]{8}{*}{ Chromosome 01} & Glyma01g02700 \\
\hline & Glyma01g02740 \\
\hline & Glyma01g04250 \\
\hline & Glyma01g09160 \\
\hline & Glyma01g21580 \\
\hline & Glyma01g21590 \\
\hline & Glyma01g21620 \\
\hline & Glyma01g39570 \\
\hline \multirow[t]{20}{*}{ Chromosome 02} & Glyma02g03420 \\
\hline & Glyma02g11610 \\
\hline & Glyma02g11620 \\
\hline & Glyma02g11630 \\
\hline & Glyma02g11640 \\
\hline & Glyma02g11650 \\
\hline & Glyma02g11670 \\
\hline & Glyma02g11680 \\
\hline & Glyma02g11690 \\
\hline & Glyma02g11700 \\
\hline & Glyma02g11710 \\
\hline & Glyma02g25930 \\
\hline & Glyma02g32020 \\
\hline & Glyma02g32770 \\
\hline & Glyma02g39080 \\
\hline & Glyma02g39090 \\
\hline & Glyma02g39680 \\
\hline & Glyma02g39700 \\
\hline & Glyma02g44100 \\
\hline & Glyma02g47990 \\
\hline \multirow[t]{16}{*}{ Chromosome 03} & Glyma03g03830 \\
\hline & Glyma03g03840 \\
\hline & Glyma03g03850 \\
\hline & Glyma03g03870 \\
\hline & Glyma03g16160 \\
\hline & Glyma03g16250 \\
\hline & Glyma03g16290 \\
\hline & Glyma03g16310 \\
\hline & Glyma03g22640 \\
\hline & Glyma03g25000 \\
\hline & Glyma03g25020 \\
\hline & Glyma03g25030 \\
\hline & Glyma03g26890 \\
\hline & Glyma03g26940 \\
\hline & Glyma03g26980 \\
\hline & Glyma03g34410 \\
\hline
\end{tabular}


Glyma03g34420

Glyma03g34440

Glyma03g34460

Glyma03g34470

Glyma03g34480

Glyma03g34490

Glyma03g41730

Chromosome 04 Glyma04g10890

Glyma04g36200

Chromosome 05

Glyma05g04200

Glyma05g25160

Glyma05g28330

Glyma05g28340

Glyma05g31500

Chromosome 06 Glyma06g33820

Glyma06g35110

Glyma06g36530

Glyma06g39350

Glyma06g40390

Glyma06g43880

Glyma06g47890

Chromosome 07 Glyma07g07320

Glyma07g07330

Glyma07g07340

Glyma07g13130

Glyma07g13560

Glyma07g14510

Glyma07g14530

Glyma07g30180

Glyma07g30190

Glyma07g30200

Glyma07g33880

Glyma07g38460

Glyma07g38470

Chromosome 08 Glyma08g07130

Glyma08g11330

Glyma08g11340

Glyma08g13230

Glyma08g19000

Glyma08g19290

Glyma08g26780

Glyma08g26790

Glyma08g26830

Glyma08g26840

Glyma08g38030

Glyma08g38060

Glyma08g38070

Glyma08g44550 


\begin{tabular}{|c|c|}
\hline & Glyma08g44690 \\
\hline & Glyma08g44700 \\
\hline & Glyma08g44710 \\
\hline & Glyma08g44720 \\
\hline & Glyma08g44730 \\
\hline & Glyma08g44740 \\
\hline & Glyma08g44750 \\
\hline & Glyma08g44760 \\
\hline & Glyma08g46270 \\
\hline & Glyma08g46280 \\
\hline & Glyma08g48240 \\
\hline Chromosome 09 & Glyma09g09910 \\
\hline & Glyma09g23310 \\
\hline & Glyma09g23330 \\
\hline & Glyma09g23600 \\
\hline & Glyma09g23720 \\
\hline & Glyma09g23750 \\
\hline & Glyma09g29160 \\
\hline & Glyma09g38130 \\
\hline & Glyma09g38140 \\
\hline & Glyma09g41690 \\
\hline & Glyma09g41700 \\
\hline Chromosome 10 & Glyma10g07090 \\
\hline & Glyma10g07110 \\
\hline & Glyma10g07160 \\
\hline & Glyma10g15730 \\
\hline & Glyma10g15790 \\
\hline & Glyma10g16790 \\
\hline & Glyma10g33790 \\
\hline & Glyma10g33800 \\
\hline & Glyma10g40900 \\
\hline & Glyma10g42680 \\
\hline Chromosome 11 & Glyma11g00230 \\
\hline & Glyma11g05680 \\
\hline & Glyma11g06880 \\
\hline & Glyma11g14260 \\
\hline & Glyma11g29480 \\
\hline & Glyma11g34720 \\
\hline & Glyma11g34730 \\
\hline Chromosome 12 & Glyma12g06220 \\
\hline & Glyma12g14050 \\
\hline & Glyma12g15870 \\
\hline & Glyma12g28270 \\
\hline & Glyma12g34030 \\
\hline Chromosome 13 & Glyma13g01220 \\
\hline & Glyma13g05580 \\
\hline & Glyma13g05590 \\
\hline & Glyma13g06170 \\
\hline
\end{tabular}


Glyma13g14190

Glyma13g21040

Glyma13g24230

Glyma13g32770

Glyma13g32910

Glyma13g36490

Glyma13g36500

Chromosome 14 Glyma14g00550

Glyma14g04790

Glyma14g04800

Glyma14g35160

Glyma14g35190

Glyma14g35220

Glyma14g35270

Glyma14g37170

Glyma14g37730

Glyma14g37770

Chromosome 15 Glyma15g05600

Glyma15g05700

Glyma15g05710

Glyma15g05980

Glyma15g06370

Glyma15g06390

Glyma15g34720

Glyma15g37520

Chromosome 16 Glyma16g03760

Glyma16g27440

Glyma16g29330

Glyma16g29340

Glyma16g29370

Glyma16g29380

Glyma16g29400

Glyma16g29420

Glyma16g29430

Chromosome 17 Glyma17g02270

Glyma17g02280

Glyma17g07340

Glyma17g14640

Glyma17g18220

Chromosome 18 Glyma18g00620

Glyma18g01950

Glyma18g03570

Glyma18g29100

Glyma18g29380

Glyma18g43980

Glyma18g44000

Glyma18g44010

Glyma18g48230 


\begin{tabular}{ll} 
& Glyma18g48250 \\
& Glyma18g50060 \\
& Glyma18g50080 \\
& Glyma18g50090 \\
& Glyma18g50100 \\
& Glyma18g50110 \\
& Glyma18g50980 \\
Chromosome 19 & Glyma19g03580 \\
& Glyma19g03610 \\
& Glyma19g03620 \\
& Glyma19g04570 \\
& Glyma19g04610 \\
& Glyma19g27600 \\
& Glyma19g37120 \\
& Glyma19g37130 \\
& Glyma19g37140 \\
& Glyma19g37150 \\
& Glyma19g37170 \\
& Glyma19g44350 \\
& Glyma20g05700 \\
& Glyma20g24360 \\
& Glyma20g26420 \\
& Glyma20g33810 \\
Ghromosome 20 & Glyma20g33820 \\
& Glyma0023s00410 \\
& Glyma0060s00320 \\
\hline Scaffold 23 &
\end{tabular}

${ }^{a}$ ORFs were identified from the Glyma1 soybean genome sequence (www. phytozome.net/soybean) by

TBLASTN of the UGT78K1 PSPG (WAPQTHVLAHDSVGVFVTHCGANSVIESVSSGVPMICRPFFGDQ).

${ }^{D}$ PSPGs without predicted ORFs or with PSPG sequence downstream of stop codon were excluded. 


\section{Appendix G}

\begin{tabular}{|c|c|}
\hline Location & Gene \\
\hline \multirow[t]{2}{*}{ Chromosome 01} & Glyma01g00730 \\
\hline & Glyma01g39460 \\
\hline Chromosome 02 & $\begin{array}{l}\text { Glyma02g11530 } \\
\text { Glyma02g39930 }\end{array}$ \\
\hline \multirow[t]{2}{*}{ Chromosome 04} & $\begin{array}{l}\text { Glyma04g36430 } \\
\text { Glyma04g40580 }\end{array}$ \\
\hline & Glyma04g40590 \\
\hline \multirow[t]{3}{*}{ Chromosome 05} & Glyma05g02810 \\
\hline & Glyma05g27960 \\
\hline & Glyma05g36210 \\
\hline \multirow[t]{8}{*}{ Chromosome 06} & $\begin{array}{l}\text { Glyma05g36220 } \\
\text { Glyma06g14200 }\end{array}$ \\
\hline & Glyma06g14210 \\
\hline & Glyma06g14220 \\
\hline & $\begin{array}{l}\text { Glyma06g18460 } \\
\text { Glyma06g43940 }\end{array}$ \\
\hline & Glyma06g43950 \\
\hline & Glyma06g43970 \\
\hline & Glyma06g44010 \\
\hline & Glyma06g45050 \\
\hline \multirow[t]{5}{*}{ Chromosome 07} & Glyma07g05460 \\
\hline & Glyma07g05470 \\
\hline & Glyma07g05480 \\
\hline & $\begin{array}{l}\text { Glyma07g05730 } \\
\text { Glyma07g06140 }\end{array}$ \\
\hline & Glyma07g33780 \\
\hline \multirow[t]{8}{*}{ Chromosome 08} & Glyma08g03430 \\
\hline & Glyma08g03440 \\
\hline & Glyma08g10930 \\
\hline & Glyma08g10940 \\
\hline & $\begin{array}{l}\text { Glyma08g18740 } \\
\text { Glyma08g27050 }\end{array}$ \\
\hline & Glyma08g27090 \\
\hline & Glyma08g27110 \\
\hline & Glyma08g27260 \\
\hline \multirow[t]{5}{*}{ Chromosome 09} & Glyma09g00590 \\
\hline & Glyma09g12440 \\
\hline & Glyma09g 12480 \\
\hline & Glyma09g41840 \\
\hline & Glyma09g41850 \\
\hline \multirow[t]{4}{*}{ Chromosome 10} & Glyma10g31990 \\
\hline & Glyma10g32010 \\
\hline & Glyma10g32020 \\
\hline & Glyma10g32030 \\
\hline
\end{tabular}




\begin{tabular}{|c|c|}
\hline & Glyma10g35980 \\
\hline \multirow[t]{2}{*}{ Chromosome 11} & $\begin{array}{l}\text { Glyma11g05800 } \\
\text { Glyma11g21080 }\end{array}$ \\
\hline & Glyma11g36410 \\
\hline \multirow[t]{4}{*}{ Chromosome 12} & Glyma12g12230 \\
\hline & Glyma12g13980 \\
\hline & Glyma13g24210 \\
\hline & Glyma13g33830 \\
\hline \multirow[t]{5}{*}{ Chromosome 14} & Glyma14g00800 \\
\hline & Glyma14g38080 \\
\hline & Glyma14g38090 \\
\hline & Glyma14g38100 \\
\hline & Glyma14g38110 \\
\hline Chromosome 15 & Glyma15g38540 \\
\hline Chromosome 16 & $\begin{array}{l}\text { Glyma16g02000 } \\
\text { Glyma16g02750 }\end{array}$ \\
\hline \multirow[t]{3}{*}{ Chromosome 17} & Glyma17g13480 \\
\hline & Glyma17g18750 \\
\hline & $\begin{array}{l}\text { Glyma17g18800 } \\
\text { Glyma18g00340 }\end{array}$ \\
\hline \multirow{4}{*}{ Chromosome 18} & Glyma18g49870 \\
\hline & Glyma18g50260 \\
\hline & $\begin{array}{l}\text { Glyma18g50280 } \\
\text { Glyma18g50290 }\end{array}$ \\
\hline & Glyma18g50470 \\
\hline \multirow[t]{3}{*}{ Chromosome 19} & Glyma19g17430 \\
\hline & Glyma19g39890 \\
\hline & $\begin{array}{l}\text { Glyma19g44490 } \\
\text { Glyma19g45000 }\end{array}$ \\
\hline \multirow[t]{10}{*}{ Chromosome 20} & Glyma20g00590 \\
\hline & Glyma20g00600 \\
\hline & Glyma20g04780 \\
\hline & Glyma20g31600 \\
\hline & Glyma20g31610 \\
\hline & Glyma20g31700 \\
\hline & Glyma20g35610 \\
\hline & Glyma20g35620 \\
\hline & Glyma20g35630 \\
\hline & Glyma20g35640 \\
\hline Scaffold 335 & Glyma0335s00200 \\
\hline
\end{tabular}

aORFs were Identified from the Glyma1 soybean genome sequence (www.phytozome net/soybean) by TBLASTN of the conserved OMT domain (PLN02589) sequence from OMT5 (KHRMSSNPVILQSVNLTKYV LETSVYPREEETLKELRKATAGHPWGFMGAAPDAGQLMTLLLKLLNAKKTIEVGVFTGYSLLLTALTIPDDGK IIALDPDREAYEIGLPFIKKAGVEHKIDFIESPALPVL KLLEDPSNKESFDFAFVDADKDNYWNYHERLLKLV).

${ }^{\mathrm{b} P S P G}$ without predicted ORFs or with the conserved OMT domain Interupted by a stop codon were 


\section{Appendix H}

Gene probe sets downregulated more than 2-fold in the seed coats of black (IRT) soybean compared to the seed coats of brown (IrT) soy- bean at the $300-400 \mathrm{mg}$ fresh seed weight stage of development ${ }^{\mathrm{a}}$

\begin{tabular}{|c|c|c|c|c|}
\hline \multirow{2}{*}{ Probe Sets } & \multirow{2}{*}{ Target Description } & \multirow{2}{*}{$\begin{array}{l}\text { Black/br } \\
\text { own fold } \\
\text { change }\end{array}$} & \multicolumn{2}{|c|}{ SAM } \\
\hline & & & P-value & d-score \\
\hline Gma 17605 1S1_at & Chalcone synthase (CHS1) & 0028 & 0000007 & -1645 \\
\hline GmaAffx 92491 1 S1_s_at & Chalcone synthase (CHS9) & 0047 & 0000005 & -1936 \\
\hline Gma 16971 2 A1_s_at & $\begin{array}{l}\text { Rıbulose-1,5-bisphosphate carboxylase } \\
\text { small subunit rbcS2 (LOC547605) }\end{array}$ & 0101 & 0000031 & -635 \\
\hline Gma 571 1 S1_at & $\begin{array}{l}\text { Weakly sımilar to A thalıana Thioredoxın } 2 \\
\text { (AtTRX2) NP } 1988111(615 \%)\end{array}$ & 0141 & 0000013 & -987 \\
\hline GmaAffx 14886 1S1_at & $\begin{array}{l}\text { Moderately similar to A thalıana branched- } \\
\text { chaın amıno acıd transamınase } 2 \\
\text { (AtBCAT-2) }(725 \%)\end{array}$ & 0172 & 0001301 & -261 \\
\hline GmaAffx 49936 1 S1_at & Transcribed locus & 0249 & 0000103 & -471 \\
\hline GmaAffx 19055 1S1_at & 5'-clone Gm-c1036-8049 & 0270 & 0000226 & -403 \\
\hline GmaAffx 89828 1 S1_s_at & $\begin{array}{l}\text { Highly similar to isoflavone reductase } \\
\text { homolog } 1 \text { (IFR1) (93\%) }\end{array}$ & 0272 & 0000132 & -446 \\
\hline Gma 34051 S1_s_at & $\begin{array}{l}\text { Moderately simılar to } A \text { thaliana branched- } \\
\text { chain amıno acıd transamınase } 2 \\
\text { (AtBCAT-2) }(725 \%)\end{array}$ & 0294 & 0003461 & -216 \\
\hline GmaAffx 77655 1 S1_at & Transcribed locus & 0306 & 0000180 & -423 \\
\hline GmaAffx 85444 1 S1_at & $\begin{array}{l}\text { Moderately sımılar to Phosphate } 2 \\
\text { ubıquitın-proteın ligase (PHO2) } \\
\text { NP_8502181(701\%) }\end{array}$ & 0326 & 0000454 & -343 \\
\hline Gma 9776 1 S1_at & Kınesın heavy chaın & 0332 & 0000023 & -783 \\
\hline Gma 1299 1S1_at & $\begin{array}{l}\text { Weakly sımılar to Phosphate } 2 \text { ubıquitın- } \\
\text { proteın ligase (PHO2) NP_850218 } 1 \\
(601 \%)\end{array}$ & 0339 & 0001125 & -269 \\
\hline Gma 7958 1 S1_at & Transcribed locus & 0341 & 0000147 & -436 \\
\hline GmaAffx 14886 1 S1_at & Kınesın heavy chaın & 0342 & 0001491 & -253 \\
\hline Gma 3801 1 S1_at & $\begin{array}{l}\text { Moderately sımılar to Phosphate } 2 \\
\text { ubıquitın-proteın ligase (PHO2) } \\
\text { NP_8502181(701\%) }\end{array}$ & 0351 & 0000036 & -595 \\
\hline GmaAffx 40574 1 S1_at & $\begin{array}{l}\text { Weakly sımilar to Populus trıchocarpa } \\
\text { predıcted proteın XP_002311637 } 1 \\
(651 \%)\end{array}$ & 0376 & 0000832 & -290 \\
\hline Soybean_rRNA_918_at & Plant $18 S$ ribosomal RNA & 0382 & 0000536 & -328 \\
\hline Gma 14852 1 S1_at & Clone JCVI-FLGm-7O7 unknown mRNA & 0388 & 0000093 & -477 \\
\hline Gma 5402 1S1_at & $\begin{array}{l}\text { Moderately sımilar to A thalıana serıne } \\
\text { carboxypeptıdase-IIke } 40 \text { (scpl40) }(714 \%)\end{array}$ & 0394 & 0000736 & -299 \\
\hline Gma 8455 1 S1_at & Flavonold 3'-hydroxylase (F3'H) & 0400 & 0000646 & -310 \\
\hline GmaAffx 93221 1 S1_s_at & $\begin{array}{l}\text { Moderately similar to Rosa hybrid cultivar } \\
\text { orcınol O-methyltransferase }(72 \%)\end{array}$ & 0410 & 0003428 & -217 \\
\hline Gma 10502 1S1_at & 3'-mRNA sequence clone Gm-r1088-1474 & 0410 & 0000368 & -359 \\
\hline GmaAffx 39265 1 S1_at & $\begin{array}{l}\text { Weakly sımilar to A thalıana antiporter/ } \\
\text { drug transporter MATE family proteın } \\
(698 \%)\end{array}$ & 0414 & 0000265 & -395 \\
\hline Gma 9164 1 S1_at & Histone H4 & 0419 & 0000602 & -316 \\
\hline GmaAffx 42587 1A1_at & $\begin{array}{l}\text { Moderate similarity to A thaliana MYB30 } \\
(715 \%)\end{array}$ & 0427 & 0000472 & -340 \\
\hline Gma 9786 1S1_at & Transcribed locus & 0433 & 0000414 & -350 \\
\hline GmaAffx 742381 S1_at & Putative RAS protein & 0439 & 0006397 & -193 \\
\hline
\end{tabular}




\begin{tabular}{|c|c|c|c|c|}
\hline GmaAffx 34868 1 A1_at & $\begin{array}{l}\text { Highly sımılar to Phaseolus coccineus } \\
\text { leucoanthocyanıdın reductase (LAR) } \\
(88 \%)\end{array}$ & 0443 & 0000016 & -902 \\
\hline Gma 13465 1 A1_at & Hypothetıcal proteın & 0444 & 0000266 & -395 \\
\hline Gma 9511 1 S1_at & Clone JCVI-FLGm-4E15 unknown mRNA & 0451 & 0000719 & -301 \\
\hline Gma 17323 1 S1_at & 5'-mRNA sequence clone Gm-r1030-2753 & 0456 & 0001666 & -248 \\
\hline GmaAffx 34657 1 A1_s_at & $\begin{array}{l}\text { Hydroxyindole-O-methyltransferase- } \\
\text { related }\end{array}$ & 0457 & 0001815 & -243 \\
\hline GmaAffx 66066 1 A1_at & $\begin{array}{l}\text { Weakly sımılar to Populus trichocarpa } \\
\text { AP2/ERF domain-containing transcription } \\
\text { factor XP_002336822 } 1(677 \%)\end{array}$ & 0459 & 0007008 & -190 \\
\hline GmaAffx 42543 1 A1_at & Transcribed locus & 0462 & 0001638 & -249 \\
\hline Gma 43402 S1_a_at & $\begin{array}{l}\text { Moderately sımilar to } P \text { trichocarpa } \\
\text { flavonold o-methyltransferase-related } \\
(739 \%)\end{array}$ & 0462 & 0004180 & -208 \\
\hline GmaAffx 40267 1 A1_at & $\begin{array}{l}\text { cDNA clone Gm-r1070-7291 3-, mRNA } \\
\text { sequence }\end{array}$ & 0466 & 0001952 & -240 \\
\hline GmaAffx 772781 A1_at & Transcribed locus clone $\mathrm{Gm}-\mathrm{r} 1088-3517$ & 0467 & 0001002 & -277 \\
\hline Gma 17559 1 S1_at & $\begin{array}{l}\text { Weakly sımılar to } A \text { thalıana heavy-metal- } \\
\text { associated domaın-contaınıng proteın } \\
(517 \%)\end{array}$ & 0469 & 0004262 & -208 \\
\hline GmaAffx 96181 S1_at & $\begin{array}{l}\text { Calcıum/calmodulın-dependent proteın } \\
\text { kınase }\end{array}$ & 0472 & 0000025 & -757 \\
\hline GmaAffx 85409 1 S1_at & $\begin{array}{l}\text { Moderately simılar to Ricinus communis } \\
\text { putative metal ion bindıng protein }(75 \%)\end{array}$ & 0474 & 0000647 & -309 \\
\hline GmaAffx 51888 1 S1_at & Putative RAD50 DNA repair proteın & 0479 & 0000889 & -286 \\
\hline GmaAffx 79131 1S1_at & Splicing factor U2AF 50 KD subunit & 0481 & 0002673 & -226 \\
\hline Gma 5949 1 A1_at & Histone $\mathrm{H} 3$ & 0483 & 0001154 & -267 \\
\hline Gma 10876 1 S1_at & $\begin{array}{l}\text { Strongly sımılar to A thalıana mannan } \\
\text { synthase/transferase (AtCSLA02) } \\
\text { NP_197666 } 1(915 \%)\end{array}$ & 0485 & 0000667 & -307 \\
\hline GmaAffx 19996 1 S1_at & IFA-bınding proteın & 0486 & 0000190 & -420 \\
\hline Gma 48842 S1_a_at & $\begin{array}{l}\text { Moderately sımılar to Citrus sinensis } \\
\text { ethylene-responsive element-bındıng } \\
\text { proteın (EREBP) mRNA }(740 \%)\end{array}$ & 0487 & 0002694 & -226 \\
\hline GmaAffx 785432 S1_at & Transcribed locus & 0487 & 0007151 & -189 \\
\hline GmaAffx 57899 1 S1_at & $\begin{array}{l}\text { Zınc finger, C3HC4 type (RING finger) } \\
\text { proteın }\end{array}$ & 0494 & 0002101 & -237 \\
\hline Gma 10949 1 S1_s_at & $\begin{array}{l}\text { Moderately sımilar to A thalıana 3- } \\
\text { chloroallyl aldehyde dehydrogenase/ } \\
\text { aldehyde dehydrogenase (NAD)/conıferyl- } \\
\text { aldehyde dehydrogenase (ALDH2C4) } \\
(755 \%)\end{array}$ & 0495 & 0000273 & -392 \\
\hline GmaAffx 7217 1 A1_at & Transcribed locus & 0496 & 0001277 & -262 \\
\hline GmaAffx 275862 S1_at & $26 \mathrm{~S}$ proteosome regulatory subunit & 0496 & 0000562 & -323 \\
\hline
\end{tabular}

${ }^{2}$ Expression values were obtained using RMA (Irizarry et al , 2003) 


\section{Appendix I}

Distribution of gene function categories of probe sets that were down-regulated more than 2-fold in the seed coat of black (iRT) soybean relative to the seed coat of brown (irT) soybean.

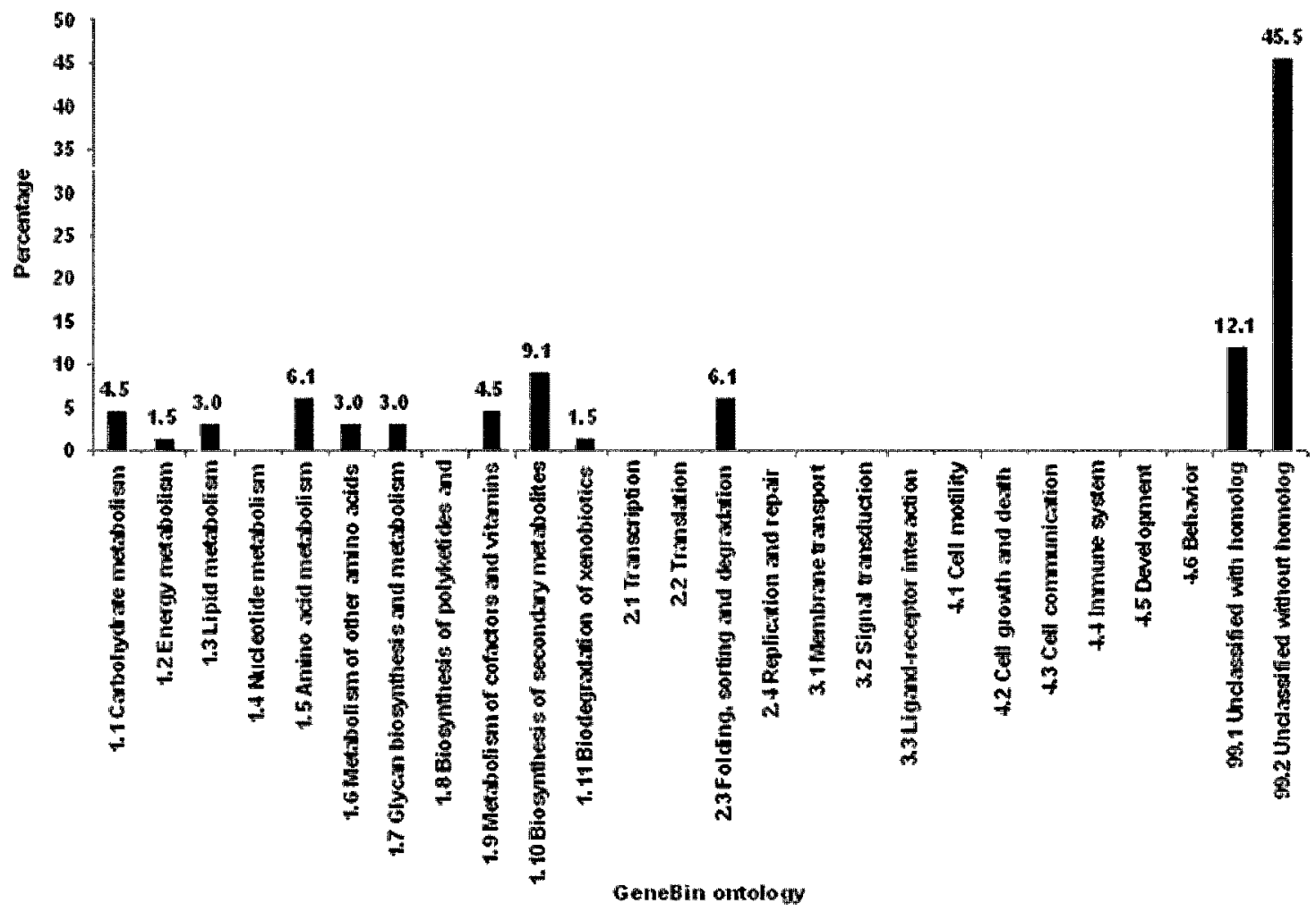




\section{Appendix J}

Genes located on chromosome 9 of the soybean genome sequence Glyma1 that are associated with probe sets differentially-regulated more than 2-fold in the seed coat of black (IRT) soybean variety Clark

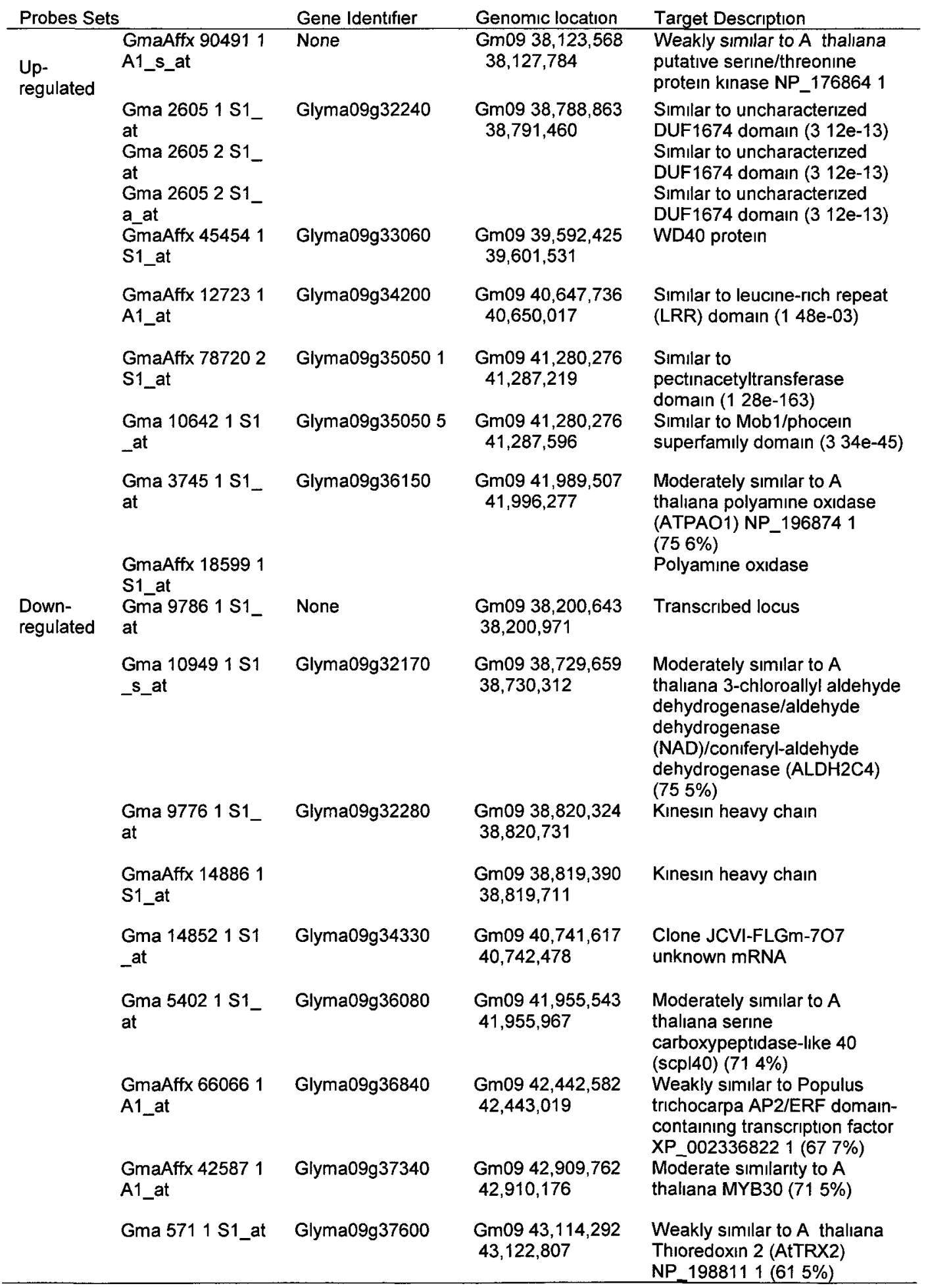




\section{Appendix K}

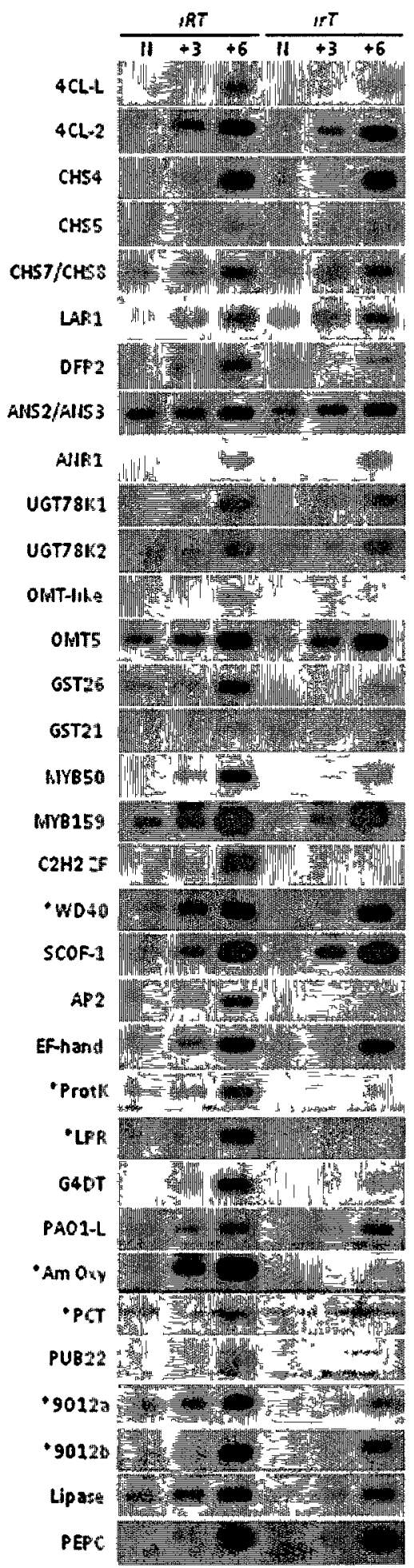

Semi-qRT-PCR validation of the expressions of select genes found to be differentially expressed in black (iRT) and brown (irT) seed coats by microarray analysis. An asterix ( $\left.{ }^{*}\right)$ 
represents genes located on Chromosome Gm09 of the soybean Glyma1 genome sequence. Genes and corresponding differentially regulated probe sets: $4 \mathrm{CL}-\mathrm{L}$, Gma.7423.2.S1_a_at; 4CL-2, Gma.8472.1.S1_at; CHS4 and CHS5, GmaAffx.42116.1.S1_at; LAR1, GmaAffx.34868.1.A1_at; DFR2, GmaAffx.80720.1.S1_at; ANS2/ANS3, Gma.1163.1.S1_at; UGT78K1, Gma.1002.2.S1_at; UGT78K2,

GmaAffx.71999.1.S1_at; OMT-like, Gma.9647.1.S1_at; OMT5, GmaAffx.57777.1.S1_at; GST26, GmaAffx.71212.1.A1_at; GST21, Gma.5139.1.S1_at; MYB50, GmaAffx.81605.1.S1_at; MYB159, GmaAffx.39483.1.A1_at; C2H2 ZF, Gma.17736.1.S1_at; WD40, GmaAffx.45454.1.S1_at; SCOF-1, Gma.235.1.S1_at; AP2, GmaAffx.2469.1.S1_at; EF-hand, Gma.15972.1.A1_at; ProtK, GmaAffx.90491.1.A1_s_at; LRR, GmaAffx.12723.1.A1_at; G4DT, Gma.5621.1.S1_at; PAO1-L, Gma.3745.1.S1_at; Am Oxy, Gma.3745.1.S1_at; PCT, GmaAffx.78720.2.S1_at; PUB22, Gma.4530.1.A1_s_at; 9012a, Gma.2605.1.S1_at; 9012b, Gma.2605.2.S1_at; Lipase, GmaAffx.90450.1.S1_at. 


\section{Appendix L}

UGT78K2 UGT78K1

UGT78K2 UGT78K1

UGT78K2 UGT78K1

UGT78K2 UGT78K1

UGT7 8K2 UGT $78 \mathrm{~K} 1$

UGT7 8K2 UGT78K1

UGT7 $8 \mathrm{~K} 2$ UGT78K1

UGT78K2 UGT78K1
MDHQNKHVAVFAFPFGSHLMPLLNLVLKLAHSLPNCSFSFIGTDKSNAILFPKPHI PNNI 60 MDHQNKHVAVFAFPFGSHLMPLLNLVLKLAHSLPNCSFSFIGTHKSNAILFPKPHI PNNI 60

KAYSISDGIPEGHVLGKNPTEKLNLFLQTGPENLHKGIELAEAETKKRVTCIVADAFVTS 120 KAYSISDGIPEGHVLGKNPTEKLNLFLQTGPENLHKGIELAEAETKKRVTCIIADAIVTS 120

SLFVAQTLNVPWIALWLPNSCSLSLYFYTËLIRQHCANHAGNTTLDFL̉PGLSKLRVEDMP 180 SLLVAQTLNVPWIALWLPNSCSLSLYFYTD̈LIRQHCASRAGNRTLDFIPGLSKLRVEDMP 180

QDLLDVGEKETVFÄRELNSLGKVLPQAKVVVMNFFEELEPPLEVQDMRSKLQSLLYVVPL 240 QDLLDVGEKETVFSRELNSLGKVLPQAKVVVMNFFEELEPPLFVQDMRNKLQSLLYVVPL 240

PSTLLPPSDTDSSGCLSWLDTKNSKSVAYVCFGTVVAPPPHELVAVAEALEESGFPFLWS 300 PSTLLPPSDTDSSGCLSWLGMKNSKSVAYVCFGTVVAPPPHELVAVAEALEESGFPFLWS 300

LKEGL/IGLLPNGFVERTKKḦGKIVSWAPQTOVLAHDSVGVFVTHCGANSVIESVSSGVPM 360 LKEGLMSLLPNGFVERTKK售GKIVSWAPQTHVLAHDSVGVFVTHCGANSVIESVSSGVPM 360

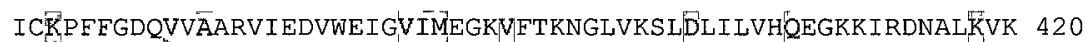
ICRPFFGDQGVVARVIEDVWEIGMMIEGKMFTKNGLVKSLNLILVHEEGKKIRDNALBVK 420

KTVEDAGRPEGQAÄQDFQTLVEVISRS 447

KTVEDAGRPEGQATQDFNTLVEVISRS 447

\section{Alignment of G. max UG3GT proteins UGT78K2 and UGT78K1 from variety Clark using the ClustalW program with default parameters. Amino acid differences are shown with grey background.}




\section{Appendix M}

\begin{tabular}{|c|c|c|c|c|}
\hline Experıment & Corresponding probe set & Target & Prımer & Sequence $\left(5^{\prime} \text { to } 3^{\prime}\right)^{\dagger \neq}$ \\
\hline \multirow[t]{44}{*}{$\begin{array}{l}\text { Semi-gRT- } \\
\text { PCR }\end{array}$} & GmaAffx 39483 1 A1_at & MYB159 & $159 \mathrm{~F}$ & TGACTCAGCCTCACCACTTG \\
\hline & & & $159 R$ & CAAAGGCATTGACCCAAATAA \\
\hline & Gma 159721 A1_at & 4CL2 & $4 \mathrm{C} 2 \mathrm{~F}$ & ACAAACATGGTGGTGGACAA \\
\hline & & & $4 \mathrm{C} 2 \mathrm{R}$ & GGTAGAAACCGCTGCCACT \\
\hline & Gma 74232 S1_a_at & $4 \mathrm{CL}$ & $4 \mathrm{CF}$ & GCAAGACCAATCTTGCATCC \\
\hline & & & $4 C R$ & TTTTGCCCAGGCATATATGTAA \\
\hline & Gma 2605 1 S1_at & $9012 b$ & $901 \mathrm{~F}$ & CACTTTGACGTCGATCCAAAC \\
\hline & & & $901 \mathrm{R}$ & GAATCAGATGTCAAACCGTTCA \\
\hline & Gma 2605 2 S1_at & $9012 a$ & $902 \mathrm{~F}$ & GCACGAGGGAAAATGTGTTT \\
\hline & & & $902 \mathrm{R}$ & GTTGGAAACGGATITGGTGT \\
\hline & & ANR1 & qAF & СССТСААТАТАAАATTССААСТGAA \\
\hline & & & qAR & CAGAGCCCCTTTGCTCTITA \\
\hline & GmaAffx 42116 1S1_at & CHS4 & CHS4F & CСTTCCAAGCCACTTTGCA \\
\hline & & & CHS4R & CTGGAGCAAAGGATGAAAGTGA \\
\hline & GmaAffx 421161 S1_at & CHS5 & CHS5F & CACTTTGCCACATTCATTCC \\
\hline & & CHS7I & CHS5R & TGTGAATGAACTAATGAAGCTATAGC \\
\hline & & CHS8 & $\mathrm{CHS78F}$ & TAGGCAAGACATGGTGGTGG \\
\hline & & & CHS78R & CTITGACTTTGGCTGGCCC \\
\hline & GmaAffx 807201S1_at & DFR2 & DFR2F & CСССТTTCTTATGCCAACAA \\
\hline & & & DFR2R & ATGTACCTCCCTTCGGCTIT \\
\hline & Gma $159721 \mathrm{~A} 1$ at & EF-Hand & EHF & ACGCCATCTCCATTCAAATC \\
\hline & & & EHR & CAGCAGGGGAAGATGAGAAG \\
\hline & Gma 5139 1S1_at & GST 21 & G21F & ACTTGATGCAGACCCCTTGT \\
\hline & & & G21R & TTCAAGAACACCCAACACCA \\
\hline & GmaAffx $712121 \mathrm{~A} 1$ at & GST1 & GST1F & CCAGGGCTTGAACATCTCAT \\
\hline & & & GST1R & TCCACGCCATGTATTTGTTG \\
\hline & GmaAffx 90491 1 A1_s_at & $\begin{array}{l}\text { Protein } \\
\text { kınase }\end{array}$ & KIF & GAAAGGTITGAACGCACCAT \\
\hline & & & KIR & ATTTTGGCGGAGTITCACAC \\
\hline & GmaAffx 732732 S1_s_at & Lipase & LIPF & CCGCATGGTTCTATCTTGGT \\
\hline & & & LIPR & TGCATTTGACAAACCCTTCA \\
\hline & GmaAffx 45454 1 S1_at & LRR & LRF & TTACATCAATGGCGGGATCT \\
\hline & & & LRR & TTGGTGGGAACTTCTTTIGG \\
\hline & GmaAffx $816051 \mathrm{~S} 1$ at & MYB50 & M50F & TITCAATCTGGAATTGGTTCG \\
\hline & & & M50R & ПТТАСТТСАТТАAССАATAАСAACAC \\
\hline & GmaAffx 57777 1S1_at & OMT5 & OMT2F & ССТСАСТGСАСТTACСАTTCC \\
\hline & & & OMT2R & TCTTTATCGGCATCAACGAA \\
\hline & Gma $96471 \mathrm{~S} 1$ at & OMT-lıke & OMTF & TTTGTTGAAAGCCAGTGCAG \\
\hline & & & OMTR & TTTTGCCTTTGTTGATGCTG \\
\hline & Gma 171411S1_at & Oxy & OXF & GCAAGTGGCTAACCGTCAAT \\
\hline & & & OXR & AGATAGTTGGAGCTAGAGTTTGAAAAA \\
\hline & & PEPC & $q P F$ & CATGCACCAAAGGGTGTTIT \\
\hline & & & qPR & TTITGCGGCAGCTATCTCTC \\
\hline & Gma 1002 2 S1_at & UGT78K1 & q3GTF & GGGTTTGTTGAGAGGACCAA \\
\hline & & & q3GTR & САТСCСAATCTCCСAAACAT \\
\hline
\end{tabular}




\begin{tabular}{|c|c|c|c|c|}
\hline & \multirow[t]{2}{*}{ GmaAffx 71999 1 S1_at } & \multirow[t]{2}{*}{ UGT78K2 } & qUAF & AAGCTGCACAGGATTTCGAC \\
\hline & & & qUAR & ACACACACACACACACACAAAAC \\
\hline & \multirow[t]{2}{*}{ Gma 5621 1 S1_at } & \multirow[t]{2}{*}{ G4DT } & $\mathrm{P} 4 \mathrm{~F}$ & GCAGACCGTTGTGTTGAAGA \\
\hline & & & P4R & CCAGGATACCAACTCCGAAA \\
\hline & \multirow[t]{2}{*}{ GmaAffx $127231 \mathrm{~A} 1 \_$at } & \multirow[t]{2}{*}{ PECTIN } & PCTF & TGAAATAAAAGCCAATCCCATT \\
\hline & & & PCTR & GATCAGTGTGCCCCAAAATC \\
\hline & \multirow[t]{2}{*}{ Gma 45301 A1_s_at } & \multirow[t]{2}{*}{ Rıng Fing } & RFF & CCATTACGGGATTGTTTGGA \\
\hline & & & RFR & TCTCCATGCATACGGAACAA \\
\hline & \multirow[t]{2}{*}{ Gma 235 1 S1_at } & \multirow[t]{2}{*}{ SCOF TF } & STFF & AGATCGAAATCCCCCAATTT \\
\hline & & & STFR & TGCAAATCTGCCACTGTTGT \\
\hline & \multirow[t]{2}{*}{ Gma 8472 1 S1_at } & \multirow[t]{2}{*}{ WD40 } & WDF & CAAGCTGGTTCAGTCGATCA \\
\hline & & & WDR & GCCTITCCTGTTCTGCAGTC \\
\hline & \multirow[t]{2}{*}{ Gma 17736 1 S1_at } & \multirow[t]{2}{*}{$\mathrm{C} 2 \mathrm{H} 2 \mathrm{ZF}$} & $\mathrm{ZF}$ & TGGACTTGGAGTTACACCTGTAGATA \\
\hline & & & ZR & CAATGATCTITATAAGGGAAAAGTCA \\
\hline & \multirow[t]{2}{*}{ Gma 4334 1 S1_at } & \multirow[t]{2}{*}{ Zinc Fing } & $\mathrm{ZF} 2 \mathrm{~F}$ & ACTGTTGGTGGTCCACGATT \\
\hline & & & ZF2R & AAGGCCAAATCCTCGTTCTT \\
\hline \multirow[t]{16}{*}{ qRT-PCR } & \multirow[t]{8}{*}{ Gma 10022 S1_at } & \multirow[t]{2}{*}{ UGT78K1 } & q3GTF & GGGTTTGTTGAGAGGACCAA \\
\hline & & & q3GTR & САТСССАAТСТСCCAAACAT \\
\hline & & \multirow[t]{2}{*}{ ANR1 } & qAF & СССТСАAТАТАAАATTССАAСТGAA \\
\hline & & & qAR & CAGAGCCCCTITGCTCTTTA \\
\hline & & \multirow[t]{2}{*}{ DFR1 } & qDF & GGGCCTGGACTTCATCACTA \\
\hline & & & qDR & АССТСССТTССАСТTСТGGT \\
\hline & & \multirow[t]{2}{*}{ GPAT } & qGPF & ATAGGATCGTGCCTGTTGCT \\
\hline & & & qGPR & CAGCCTTAACCGACATCTCC \\
\hline & \multirow[t]{4}{*}{ GmaAffx 34868 1 A1_at } & \multirow[t]{2}{*}{ LAR1 } & $\mathrm{qL} 1 \mathrm{~F}$ & TAGGCTTTGGTGAGGAGGAA \\
\hline & & & qL1R & TGACACCAAAACAGAGTGACAA \\
\hline & & \multirow[t]{2}{*}{ PEPC } & qPF & CATGCACCAAAGGGTGTTTT \\
\hline & & & qPR & TIITGCGGCAGCTATCTCTC \\
\hline & \multirow[t]{2}{*}{ GmaAffx 71999 1 S1_at } & \multirow[t]{2}{*}{ UGT78K2 } & qUAF & AAGCTGCACAGGATTTCGAC \\
\hline & & & qUAR & ACACACACACACACACACAAAAC \\
\hline & \multirow[t]{2}{*}{ GmaAffx 57777 1 S1_at } & \multirow[t]{2}{*}{ OMT5 } & $\mathrm{OMT} 2 \mathrm{~F}$ & ССТСАСТGСАСТTАССАТTCC \\
\hline & & & OMT2R & TCTITATCGGCATCAACGAA \\
\hline \multirow[t]{2}{*}{$\begin{array}{l}\text { cDNA } \\
\text { clonıng }\end{array}$} & GmaAffx 57777 1 S1_at & OMT5 & $\begin{array}{l}\text { HO5F } \\
\text { HO5R }\end{array}$ & $\begin{array}{l}\text { CACCcatatgGCCGAAGAGGAGAGGCAT } \\
\text { ACTCctcgagCCCCACTCCCAAAAGAGA } \\
\text { GAAAAA }\end{array}$ \\
\hline & GmaAffx 71999 1 S1_at & UGT78K2 & $\begin{array}{l}\text { UHF } \\
\text { UAR }\end{array}$ & $\begin{array}{l}\text { GTAGCA } \\
\text { ACTCggatcCACACACACACACACACAC } \\
\text { AAAACATAAAAGT }\end{array}$ \\
\hline
\end{tabular}

${ }^{\dagger}$ Restriction site in lowercase

${ }^{\ddagger}$ Start codon underlıned 


\section{Appendix $\mathbf{N}$}

Primers.

\begin{tabular}{|c|c|c|c|}
\hline Experiment & Target & Primer & Sequence $\left(5^{\prime} \text { to } 3^{\prime}\right)^{t, \neq}$ \\
\hline $\begin{array}{l}\text { 5'-RACE of } \\
\text { ANR }\end{array}$ & EV264997 & AEV1R & GCAAAGTTCACAGGTGTAGCAAGCTGGA \\
\hline \multirow{3}{*}{$\begin{array}{l}\text { 3'-RACE of } \\
\text { ANR }\end{array}$} & BM092813 & AEB4R & GAGCACAGCAAATGTATCGACCAGAAGC \\
\hline & EV264997 & AEV1F & GATTGGAGGGAGTGGATTTATGGCCTCT \\
\hline & BM092813 & $\mathrm{AB} 4 \mathrm{~F}$ & GCTTCTGGTCGATACATTTGCTGTGCTC \\
\hline \multirow[t]{12}{*}{ qRT-PCR } & ANR1 & $\mathrm{qAF}$ & СССТСAATATAAAATTCCAACTGAA \\
\hline & & qAR & CAGAGCCCCTTTGCTCTTTA \\
\hline & ANR2 & $\mathrm{qA} 2 \mathrm{~F}$ & AAGCAAATCGGAAAGAAGCA \\
\hline & & $\mathrm{qA} 2 \mathrm{R}$ & TCAATTCGCCCAGATTTTTC \\
\hline & UGT78K2 & qUAF & AAGCTGCACAGGATTTCGAC \\
\hline & & qUAR & ACACACACACACACACACAAAAC \\
\hline & ANS2/ANS3 & $\mathrm{A} 23 \mathrm{~F}$ & CTGAGCAACGGCAAGTACAA \\
\hline & & A23R & CAATTTGGGAGACCTTCCTG \\
\hline & LAR1 & $q L 1 F$ & TAGGCTTTGGTGAGGAGGAA \\
\hline & & qL1R & TGACACCAAAACAGAGTGACAA \\
\hline & PEPC16 & qPF & CATGCACCAAAGGGTGTTTT \\
\hline & & qPR & TTTTGCGGCAGCTATCTCTC \\
\hline \multirow[t]{4}{*}{$\begin{array}{l}\text { cDNA } \\
\text { cloning }\end{array}$} & ANR1 & AHF & CACCcatATGGCCGCTATCAAGCCAACTG \\
\hline & & AHR & ACTCggatccACAGAGATGGTTGCTATTCCCCTTGATT \\
\hline & ANR2 & $\mathrm{AH} 2 \mathrm{~F}$ & CACCcatATGGCCAATATCAAGCAAATCG \\
\hline & & $\mathrm{AH} 2 \mathrm{R}$ & ACTCggatccTTAGTTGAGCAGGGCCCCC \\
\hline \multirow{2}{*}{$\begin{array}{l}\text { Genomic } \\
\text { cloning }\end{array}$} & ANR1 & OGF & CACCGAATTCCTTCTCTCCTGAGGGAGGATT \\
\hline & & OGR & ACTCGGATCCTCCAAACTTAATAAAGCGACTGA \\
\hline
\end{tabular}

${ }^{\dagger}$ Restriction site underlined.

${ }^{\ddagger}$ Start codon bold. 


\section{Appendix 0}

Primers.

\begin{tabular}{|c|c|c|c|}
\hline Experiment & Target & Primer & Sequence $\left(5^{\prime} \text { to } 3^{\prime}\right)^{\dagger}$ \\
\hline $\begin{array}{l}\text { PCR } \\
\text { genotyping }\end{array}$ & GUS linker & GUSF & CATGAAGATGCGGACTTACG \\
\hline $\begin{array}{l}\text { ANR-RNAi } \\
\text { cloning }\end{array}$ & ANR1 3'-CDS & $\begin{array}{l}\text { GUSR } \\
\text { ASF } \\
\text { ASR }\end{array}$ & $\begin{array}{l}\text { ATCCACGCCGTATTCGG } \\
\text { CACCGGTACCCTCTCACAACTGGTCCTTCTG } \\
\text { TCACTAC } \\
\text { AGTTGGATCCGCTGAGAAACTTTGCAAGCTC } \\
\text { AGG }\end{array}$ \\
\hline \multirow[t]{20}{*}{ qRT-PCR } & ANR1 & qAF & СССТСААТАТАAАATTCСАACTGAA \\
\hline & & qAR & CAGAGCCCCTTTGCTCTTTA \\
\hline & ANR2 & qA2F & AAGCAAATCGGAAAGAAGCA \\
\hline & & qA2R & TCAATTCGCCCAGATTTTTC \\
\hline & ANS2/ANS3 & A23F & CTGAGCAACGGCAAGTACAA \\
\hline & & A23R & CAATTTGGGAGACCTTCCTG \\
\hline & $D F R 1$ & qDF & GGGCCTGGACTTCATCACTA \\
\hline & & qDR & ACСTCССTTCCACTTCTGGT \\
\hline & GST26 & GST1F & CCAGGGCTTGAACATCTCAT \\
\hline & & GST1R & TCCACGCCATGTATTTGTTG \\
\hline & GUS linker & qGF & CCCTTACGCTGAAGAGATGC \\
\hline & & qGR & GAGGTTAAAGCCGACAGCAG \\
\hline & LAR1 & qL1F & TAGGCTTTGGTGAGGAGGAA \\
\hline & & $q L 1 R$ & TGACACCAAAACAGAGTGACAA \\
\hline & PEPC16 & qPF & CATGCACCAAAGGGTGTTTT \\
\hline & & qPR & TTTTGCGGCAGCTATCTCTC \\
\hline & UGT78K1 & q3GTF & GGGTTTGTTGAGAGGACCAA \\
\hline & & q3GTR & САТСССААТСТСССАAАCAT \\
\hline & UGT78K2 & qUAF & AAGCTGCACAGGATTTCGAC \\
\hline & & qUAR & ACACACACACACACACACAAAAC \\
\hline
\end{tabular}

${ }^{\dagger}$ Restriction site underlined. 


\section{Appendix P}

Multiple reacton monitoring specifications for quantificaton of differentially accumulated flavonoid compounds from the seed coats of transgenic soybeans expressing the ANR-RNAi construct using a 3200 QTRAP $^{\dagger}$.

\begin{tabular}{lcccccccc}
\hline Compound & Q1 & Q3 & $\begin{array}{l}\text { Time } \\
(\mathrm{msec})\end{array}$ & $\begin{array}{l}\text { DP } \\
\text { (Volts) }\end{array}$ & $\begin{array}{l}\text { EP } \\
\text { (Volts) }\end{array}$ & $\begin{array}{l}\text { CEP } \\
\text { (Volts) }\end{array}$ & $\begin{array}{l}\text { CE } \\
\text { (Volts) }\end{array}$ & $\begin{array}{l}\text { CXP } \\
\text { (Volts) }\end{array}$ \\
\hline $\begin{array}{l}(-)- \\
\text { Epicatechin }\end{array}$ & 291.0 & 139.1 & 20 & 26 & 6 & 26 & 21 & 4 \\
Isorhamnetin- & 479.0 & 123.1 & 20 & 26 & 6 & 26 & 35 & 4 \\
3-O- & 317.1 & 20 & 16 & 6 & 24 & 25 & 6 \\
glucoside & 479.0 & 85.1 & 20 & 16 & 6 & 24 & 39 & 4 \\
Procyanidin & 579.8 & 126.9 & 20 & 36 & 7 & 30 & 35 & 4 \\
B2 & 579.8 & 123.0 & 20 & 36 & 7 & 30 & 75 & 4 \\
Quercetin-3- & 465.0 & 303.2 & 20 & 21 & 4 & 24 & 21 & 4 \\
O-glucoside & 465.0 & 85.1 & 20 & 21 & 4 & 24 & 45 & 4 \\
Cyanidin-3- & 449.0 & 288.1 & 20 & 70 & 10 & 23 & 50 & 3 \\
O-galactoside & 449.0 & 287.2 & 20 & 70 & 10 & 23 & 50 & 3 \\
Cyanidin-3- & 449.0 & 288.2 & 20 & 70 & 10 & 23 & 50 & 3 \\
O-glucoside & 449.0 & 287.1 & 20 & 70 & 10 & 23 & 50 & 3 \\
\hline
\end{tabular}

${ }^{\dagger}$ See Materials and Methods section for detailed description of the LC-MS/MS system. 
Appendix Q

Developmental properties of T2 lines compared to the untransformed control (ir) at two stages of development.

\begin{tabular}{|c|c|c|c|c|c|}
\hline Property & ir & A1A1-1-2 & A1A1-2-2 & A1A1-3-4 & $\mathrm{A} 1 \mathrm{~A} 1-4-4$ \\
\hline \multicolumn{6}{|l|}{$25-50 \mathrm{mg}$ FSW } \\
\hline Days post-anthesis & 30 & 30 & 30 & 30 & 30 \\
\hline $\begin{array}{l}\text { Embryo morphology } \\
\text { GPAT relative } \\
\text { expression }\end{array}$ & $\begin{array}{c}\text { cotyledon } \\
4.371 \pm \\
0.19\end{array}$ & $\begin{array}{c}\text { cotyledon } \\
5.357 \pm \\
0.64\end{array}$ & $\begin{array}{c}\text { cotyledon } \\
3.950 \pm \\
0.97\end{array}$ & $\begin{array}{c}\text { cotyledon } \\
4.430 \pm \\
0.29\end{array}$ & $\begin{array}{c}\text { cotyledon } \\
4.636 \pm \\
0.58\end{array}$ \\
\hline T-Test ${ }^{b}$ & N/A & 0.104 & 0.41 & 0.802 & 0.524 \\
\hline \multicolumn{6}{|l|}{$400-500 \mathrm{mg}$ FSW } \\
\hline $\begin{array}{l}\text { Days post-anthesis } \\
\text { Embryo morphology } \\
\text { GPAT relative } \\
\text { expression }{ }^{a}\end{array}$ & $\begin{array}{c}40 \\
\text { early } \\
\text { maturation } \\
0.639 \pm \\
0.04\end{array}$ & $\begin{array}{c}40 \\
\text { early } \\
\text { maturation } \\
0.672 \pm \\
0.02\end{array}$ & $\begin{array}{c}40 \\
\text { early } \\
\text { maturation } \\
0.631 \pm \\
0.04\end{array}$ & $\begin{array}{c}40 \\
\text { early } \\
\text { maturation } \\
0.635 \pm \\
0.04\end{array}$ & $\begin{array}{c}40 \\
\text { early } \\
\text { maturation } \\
0.650 \pm \\
0.05\end{array}$ \\
\hline T-Test $^{\mathrm{b}}$ & N/A & 0.271 & 0.838 & 0.917 & 0.759 \\
\hline
\end{tabular}

${ }^{a} \mathrm{qRT}-\mathrm{PCR}$ relative to $P E P C 16$ using seed coat $\mathrm{CDNA}$.

${ }^{\text {'T } T w o-t a i l e d ~ s t u d e n t ' s ~} t$ test compared to the untransformed control (ir). 


\section{Appendix $\mathbf{R}$}

\begin{tabular}{|c|c|c|}
\hline Gene & $\begin{array}{c}\text { Genbank } \\
\text { Accession }\end{array}$ & $\begin{array}{l}\text { Percent } \\
\text { Identity }\end{array}$ \\
\hline ANR1 & JF433915 & 100 \\
\hline ANR2 & JN098512 & 86 \\
\hline LAR1 & JF433916 & 55 \\
\hline$D F R 1$ & AF167556 & 53 \\
\hline$D F R 2$ & DQ026299 & 51 \\
\hline IFR1L & JF433916 & 47 \\
\hline
\end{tabular}

